\title{
The ecology of \\ Lyme borreliosis risk
}

Interactions between Ixodes ricinus, rodents and Borrelia burgdorferi sensu lato 


\section{The ecology of \\ Lyme borreliosis risk}

Interactions between Ixodes ricinus, rodents

and Borrelia burgdorferi sensu lato

Gilian van Duijvendijk 


\section{Thesis committee}

\section{Promotor}

Prof. Dr W. Takken

Personal chair at the Laboratory of Entomology

Wageningen University

\section{Co-promotor}

Dr H. Sprong

Research coordinator wildlife and vector-borne diseases

National Institute for Public Health and the Environment, Bilthoven

\section{Other members}

Prof. Dr H.F.J. Savelkoul, Wageningen University

Prof. Dr K. Verheyen, Ghent University, Belgium

Dr W.F. de Boer, Wageningen University

Dr M.J. Voordouw, Université of Neuchâtel, Switzerland

This research was conducted under the auspices of the C. T. de Wit Graduate School for Production Ecology and Resource Conservation 


\title{
The ecology of \\ Lyme borreliosis risk
}

\author{
Interactions between Ixodes ricinus, rodents \\ and Borrelia burgdorferi sensu lato
}

\section{Gilian van Duijvendijk}

Thesis

submitted in fulfilment of the requirements for the degree of doctor

at Wageningen University

by the authority of the Rector Magnificus

Prof. Dr A.P.J. Mol,

in the presence of the

Thesis Committee appointed by the Academic Board

to be defended in public

on Tuesday 20 December 2016

at 11 a.m. in the Aula 
Gilian van Duijvendijk

The ecology of Lyme borreliosis risk: Interactions between Ixodes ricinus, rodents and Borrelia burgdorferi sensu lato 188 pages.

PhD thesis, Wageningen University, Wageningen, NL (2016)

With references, with summary in English

ISBN 978-94-6257-940-8

DOI 10.18174/390911 




\section{Table of contents}

Abstract

Chapter 1: General introduction

Chapter 2: Multi-trophic interactions driving the transmission cycle of Borrelia afzelii between Ixodes ricinus and rodents: A review

Chapter 3: Rodent density affects Lyme borreliosis risk by affecting the density of Ixodes ricinus nymphs, but not their infection rate with Borrelia burgdorferi sensu lato

Chapter 4: Interactions between Ixodes ricinus, rodents and Borrelia burgdorferi sensu lato affecting Lyme borreliosis risk in a natural ecosystem

Chapter 5: A Borrelia afzelii infection increases larval tick burden on Myodes glareolus and nymphal body weight of Ixodes ricinus

Chapter 6: Rodents as hosts for Ixodes ricinus and Borrelia afzelii

Chapter 7: Behavioural responses of Ixodes ricinus nymphs to carbon dioxide and rodent odour

Chapter 8: Larvae of Ixodes ricinus transmit Borrelia afzelii and

B. miyamotoi to vertebrate hosts

Chapter 9: General discussion

References

Summary

Appendices

Acknowledgements

Curriculum vitae 



\section{Abstract}

The sheep tick (Ixodes ricinus) is widespread throughout Europe and can transmit Borrelia burgdorferi sensu lato (s.l.), which can cause Lyme borreliosis and B. miyamotoi, the agent of Borrelia miyamotoi disease in humans. Borrelia afzelii is the most common genospecies of Borrelia burgdorferi s.I. in western Europe and it circulates between I. ricinus and rodents. The density of infected nymphs determines disease risk and is affected by complex multi-trophic interactions between factors that are not well understood. The aim of this thesis was, therefore, to gain insight into the multi-trophic interactions that affect the density of $B$. afzelii-infected and B. miyamotoi-infected $I$. ricinus nymphs.

Interactions between $I$. ricinus, rodents (wood mice and bank voles) and $B$. afzelii were reviewed to elucidate knowledge gaps concerning these interactions. The effect of rodent density on the density of infected nymphs and the factors that affect the contribution of a rodent to the density of infected nymphs were investigated in field experiments. The effects of a $B$. afzelii infection on larval tick burden of bank voles and nymphal body weight were investigated in the laboratory. The effects of $\mathrm{CO}_{2}$ and host odour on the host-seeking behaviour of $I$. ricinus were investigated in a Y-tube olfactometer. Finally, the ability of field-collected $I$. ricinus larvae to transmit $B$. afzelii and $B$. miyamotoi was analysed.

The review showed that rodent density, rodent infection rate, and larval tick burden are the major factors affecting the density of $B$. afzelii-infected nymphs one year later. The field experiments showed that rodent density can positively affect the density of $B$. burgdorferi s.l.infected nymphs one year later. However, the effect of rodent density was small when the density of infected nymphs was limited by the density of questing larvae in the previous year. Rodent density had, however, no effect on the density of $B$. miyamotoi-infected nymphs. The field studies also showed that tick burdens are 2.4 times higher on wood mice than on bank voles and that body weight of emerged nymphs was $36 \%$ higher for ticks that fed on wood mice compared to ticks that fed on bank voles. Bank voles infected with $B$. afzelii acquired a larger larval tick burden, resulting in a $27 \%$ increase in nymphal infection prevalence. Furthermore, infected nymphs had a $10 \%$ greater the body weight. Ixodes ricinus nymphs are activated by but not attracted to $\mathrm{CO}_{2}$, whereas they are attracted to and not activated by host odour. Ixodes ricinus larvae transmitted both $B$. afzelii and $B$. miyamotoi to rodents and it was calculated that rodents have a chance of about $25-75 \%$ of acquiring $B$. afzelii from larvae relative to the chance of acquiring $B$. afzelii from nymphs. The main conclusions of this thesis are discussed and suggestions are made for future research. 



\section{Chapter 1}

\section{General introduction}




\section{Introduction}

The sheep tick (Ixodes ricinus) is widespread throughout Europe and can transmit a variety of medically and veterinary important pathogens, including Borrelia burgdorferi sensu lato (s.l.), which can cause Lyme borreliosis in humans and B. miyamotoi, the pathogenic agent of Borrelia miyamotoi disease in humans. In The Netherlands, the incidence of Lyme borreliosis has increased in the past decades from 4 per patients with erythema migrans per 10,000 inhabitants in 1994 to 13 per 10,000 inhabitants in 2009 (Hofhuis et al. 2015). This increase is expected to be the result of an increase in the activity and abundance of infected $I$. ricinus nymphs (Sprong et al. 2012, Medlock et al. 2013). Borrelia miyamotoi has only recently been identified as a human pathogen and although it is globally widespread, little is known about the prevalence and incidence of Borrelia miyamotoi disease (Siński et al. 2016).

Ixodes ricinus is a generalist tick species that feeds on a variety of vertebrate hosts. It hatches from the egg as a larva (Figure 1). This larva quests for a host in the vegetation. Wood mice and bank voles are commonly used hosts by I. ricinus larvae. The larva ingests blood for a few days, drops from the host to digest its blood meal and, after an obligatory development time in the leaf litter, moults into a nymph, which quests for another vertebrate host. A nymph can feed on a variety of host species, ingests blood for a few days, drops from the host to digest its blood meal to moult into an adult. Adult females quest for another vertebrate host, usually a large mammal to ingest blood, drop from the host to digest the blood and lay eggs, after which they die. The lifecycle takes about four years, most of which is spent in the vegetation.

Borrelia burgdorferi s.l. consists of several different genospecies (Stanek et al. 2012), which can cause different clinical manifestations in humans and circulate in different enzootic lifecycles. Genospecies present in The Netherlands include B. afzelii, B. bavariensis $B$. burgdorferi sensu stricto and Borrelia spielmanii, which are associated to rodents, $B$. garinii and $B$. valaisiana, which are associated to birds and $B$. lusitaniae, which is associated to lizards (Hu et al. 1997a, Huegli et al. 2002, Kurtenbach et al. 2002a, Hanincová et al. 2003a, Hanincová et al. 2003b, Michalik et al. 2005, Richter and Matuschka 2006, Richter et al. 2011, Norte et al. 2015, Coipan et al. 2016). All genospecies can be transmitted by I. ricinus. In western Europe, $B$. afzelii is the most common genospecies found in ticks (Rauter and Hartung 2005, Gassner et al. 2011) and, as a result, causes most of the cases of Lyme borreliosis in humans (Coipan et al. 2016). Infected rodents stay infectious to feeding larvae throughout their life (Gern et al. 1994) and the infection has no effect on rodent survival (Voordouw et al. 2015). When hatched from the eggs, the larva of $I$. ricinus is free of $B$. afzelii (Richter et al. 2012), but it can acquire the infection during blood feeding on an infected rodent. Larvae that acquired a $B$. afzelii infection from an infected rodent moult into infected nymphs, which can infect other rodents during their blood meal. The nymphal stage is also thought to be most responsible for 
infecting humans (Radolf et al. 2012) as this is the life stage most often encountered on humans. The density of infected nymphs, therefore, determines the acarological risk, which often associates with the incidence of Lyme borreliosis (Stafford III et al. 1998, Diuk-Wasser et al. 2012).

Wood mice (Apodemus sylvaticus) and bank voles (Myodes glareolus) are the most common rodent species in The Netherlands (Figure 2) (Gassner et al. 2013) and are commonly used as hosts by larval $I$. ricinus. Even though the Dutch woodlands are home to a large variety of mammal and bird species (e.g. Apodemus sylvaticus, Myodes glareolus, Erinaceus europaeus, Vulpes vulpes, Capreolus capreolus, Cervus elaphus, Sus scrofa, Parus major, Cyanistes caeruleus, Erithacus rubecula, Fringilla coelebs, Phylloscopus collybita, Sylvia atricapilla, Turdus merula, Turdus philomelos), due to their high abundance, wood mice and bank voles feed the majority of I. ricinus larvae (Hofmeester et al. 2016). These rodents species differ, however, in their suitability as hosts for ticks and spirochaetes. Wood mice have greater tick burdens and higher infections rates than bank voles, but the chance that an uninfected larva acquires the infection is larger when feeding on a bank vole (Humair et al. 1993, Kurtenbach et al. 1994, Tälleklint and Jaenson 1994, Kurtenbach et al. 1995, Tälleklint and Jaenson 1997, Gray et al. 1999, Humair et al. 1999, Kybicova et al. 2008, Gassner et al. 2013, Radzijevskaja et al. 2013). These interactions between I. ricinus, rodents and $B$. afzelii affect the contribution of wood mice and bank voles to Lyme borreliosis risk but are not well understood. This thesis, therefore, studied the interactions between I. ricinus, rodents (wood mice and bank voles) and $B$ afzelii. Borrelia afzelii was used as a proxy for B. burgdorferi s.l. infections. Detailed knowledge of the ecology of B. burgdorferi s.l. and B. miyamotoi helps to understand how these spirochaetes circulate in their enzootic lifecycles.

The aim of this thesis was to gain insight in the multi-trophic interactions that affect the density of $B$. burgdorferi s.I.-infected and B. miyamotoi-infected I. ricinus nymphs. 


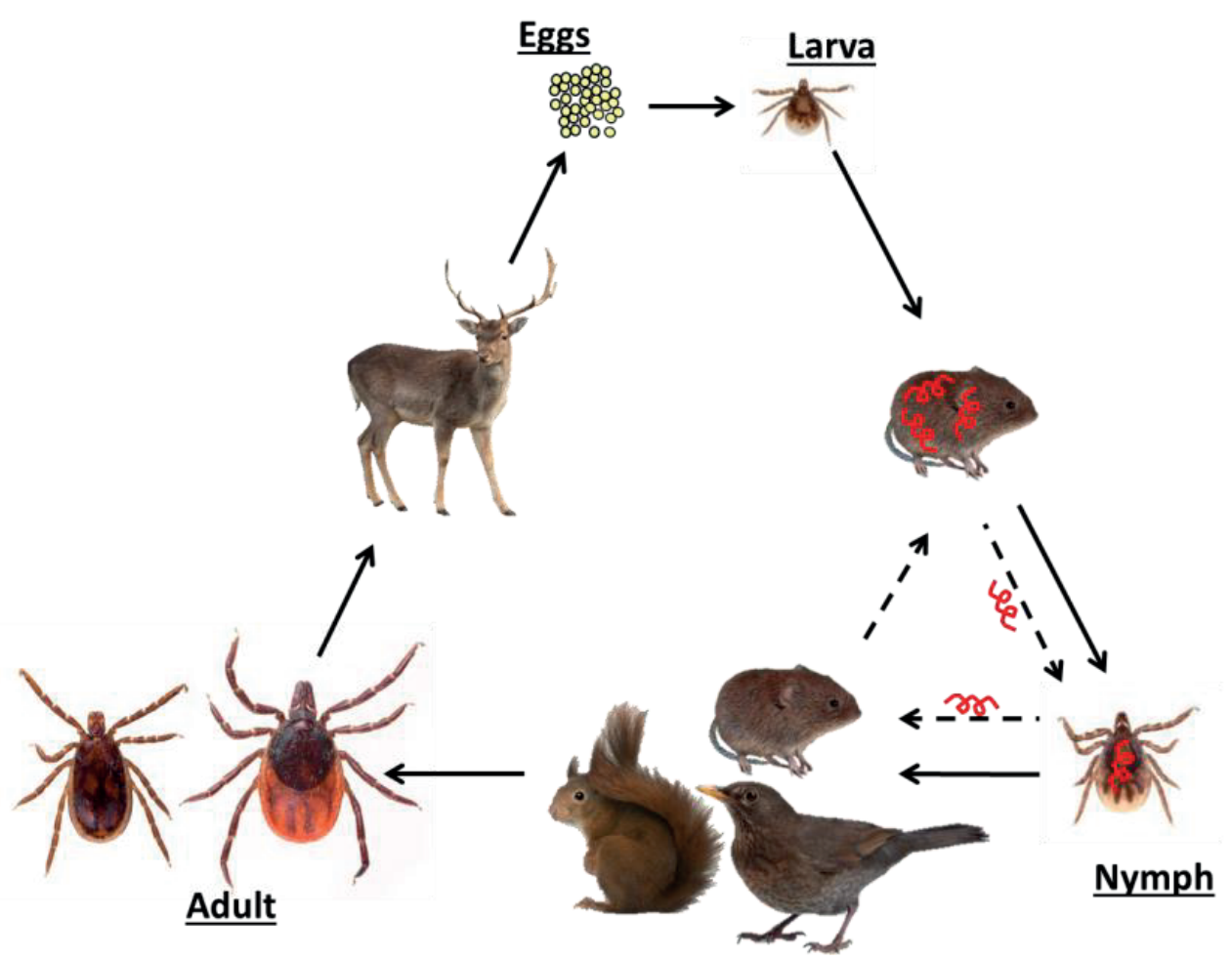

Figure 1. Schematic overview of the lifecycle of Ixodes ricinus (solid lines) and Borrelia afzelii (dotted lines). Pictures of the ticks: Hans Smid.

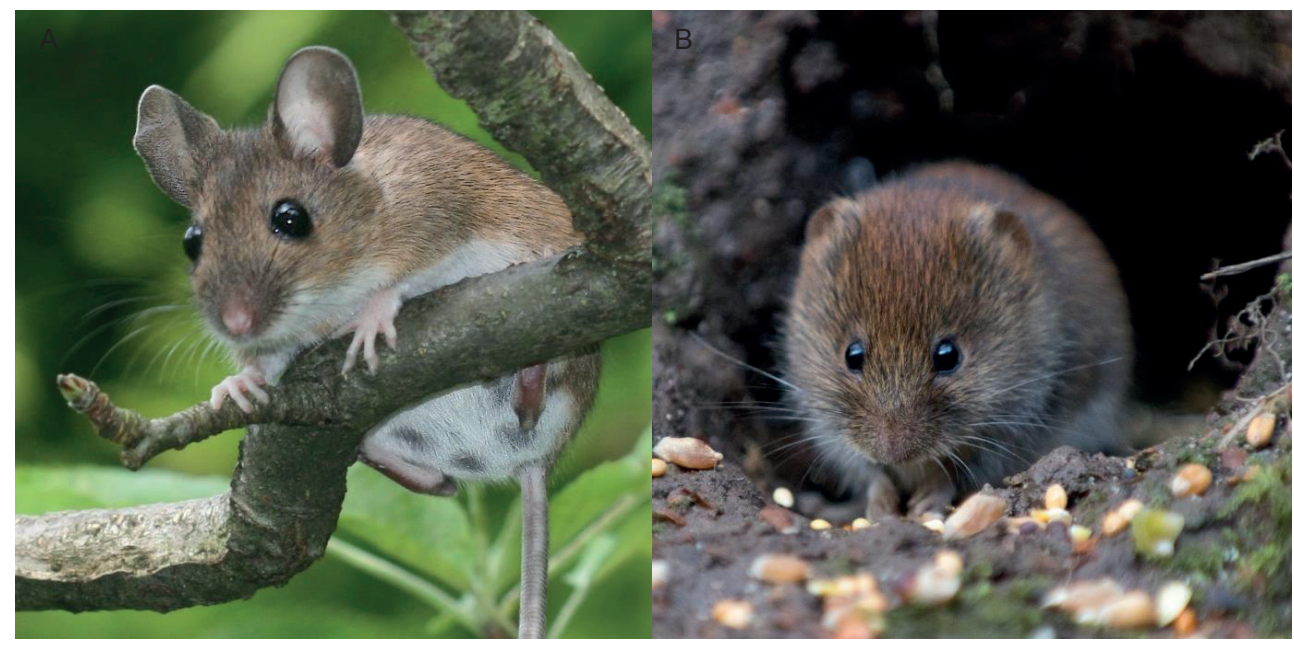

Figure 2. Rodent species of The Netherlands that are commonly selected as blood host by Ixodes ricinus larvae and as reservoir host for Borrelia afzelii. (A) Wood mouse (Apodemus sylvaticus) and (B) bank vole (Myodes glareolus). Photos by Hans Hillewaert (A. sylvaticus, CC BY-SA 3.0) and Evan James Shymko (M. glareolus, CC BY 3.0). 


\section{Thesis outline}

Chapter 2 reviews the current knowledge on the multi-trophic interactions that drive the transmission cycle of $B$. afzelii between I. ricinus and rodents. The incidence of Lyme borreliosis is affected by the density of infected nymphs. This density is affected by the chance that a questing tick larva develops into a questing infected nymph. This development depends on success of 1) larval host finding, 2) larval blood feeding, 3) spirochaete transmission from rodent to larva, and 4) development from larva to nymph. Nymphal host finding success, nymphal blood feeding success, and spirochaete transmission from nymph to rodent are the final steps to complete the enzootic $B$. afzelii lifecycle and are included in the review. A literature-based overview of the effects from the side of the rodent, tick and Borrelia pathogen on these steps is discussed.

Wood mice and bank voles are used as host by I. ricinus larvae and serve as reservoir host for $B$. afzelii. In Chapter 3, I studied the effect of rodent density on the density of infected nymphs. This effect was studied in a field experiment in a forest near Wageningen, The Netherlands (Figure 3). Rodent density was manipulated by either removing rodents from plots to reduce rodent density, adding acorns as rodent food to increase rodent density, and the control plots received no treatment. Rodent densities and rodent infections were measured for 2.5 years and tick densities and infections were measured for 3.5 years.

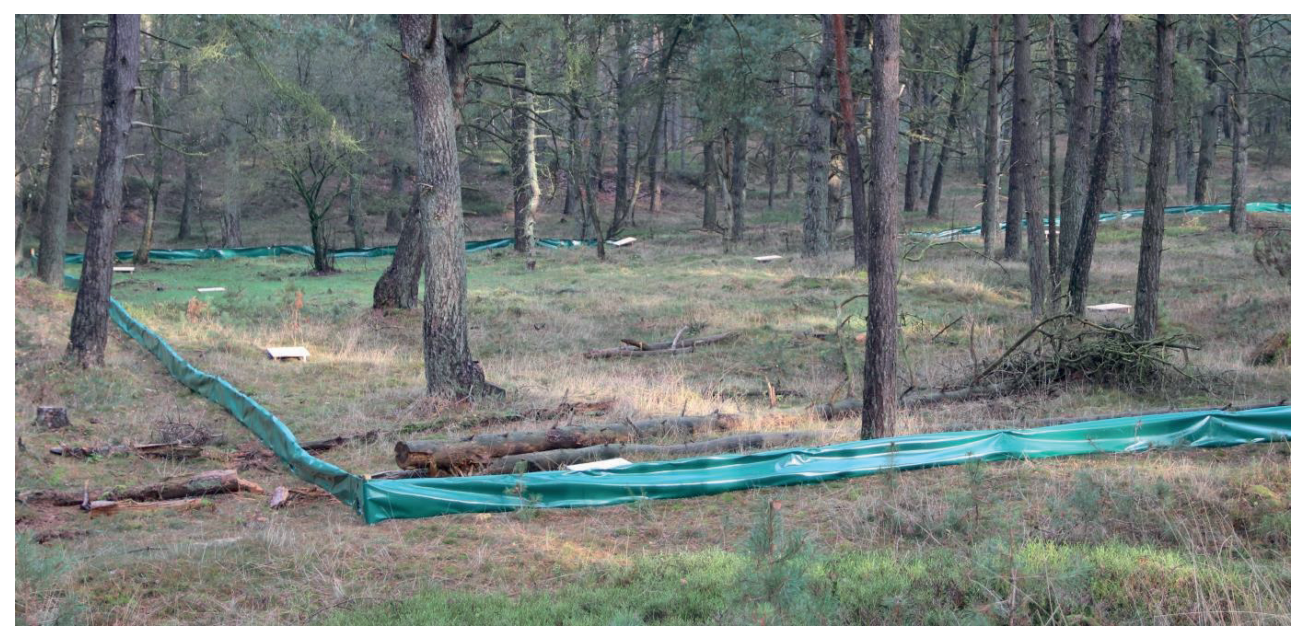

Figure 3. Experimental plot of the field experiment to study the effect of rodent density of the density of infected nymphs, described in Chapter 3. 
Rodents are important for the lifecycles of $I$. ricinus and $B$. afzelii. Not all rodents contribute equally to the density of infected nymphs. In Chapter 4, I studied the interactions between rodents, I. ricinus and B. burgdorferi s.I. in a natural ecosystem to determine the factors that affect the contribution of a rodent to the density of infected nymphs (Figure 4). These interactions were studied in another forest near Wageningen, The Netherlands. Rodents were trapped at two locations. Rodent species, sex, weight, identification number, location, time, infection, infectivity and tick burden were determined.

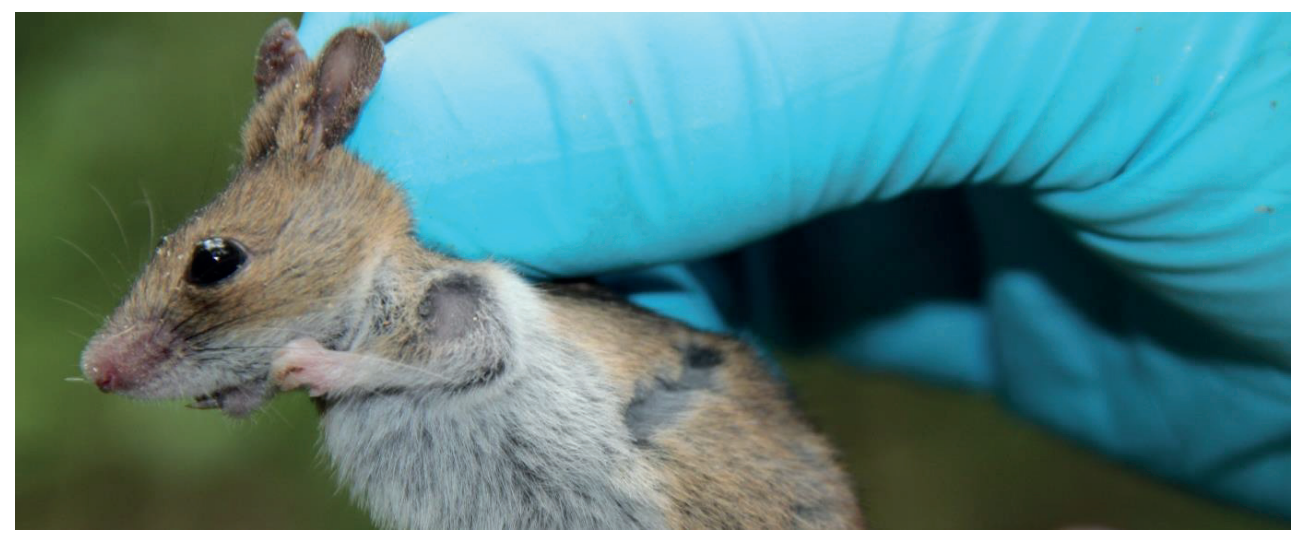

Figure 4. Trapped and marked wood mouse with attached Ixodes ricinus larvae (ears) during the field experiment to identify the characteristics of the rodents that contribute most to the density of infected nymphs, described in Chapter 4 .

There are several examples of microorganisms that affect their host or vector to enhance their own transmission. An increased larval tick burden on $B$. afzelii-infected rodents enhances the lifecycle of $B$. afzelii. Ticks are not evenly spread among rodents. In Chapter 5, I investigated whether this heterogeneity could be explained by the infection status of the rodent, to elucidate if $B$. afzelii affects its own transmission. Bank voles were inoculated with $B$. afzelii or control medium after which host preference of $I$. ricinus nymphs was determined in a Y-tube olfactometer (Figure 5). Thereafter, infected and uninfected bank voles were placed in a semifield arena containing questing larvae to determine their acquisition of larval tick burdens to investigate whether a $B$. afzelii infection had an effect on larval tick burden (and therefore transmission from rodent to tick). Engorged larvae were collected from these bank voles, allowed to moult into nymphs, weighed and analysed for infection to determine whether a $B$. afzelii infection had an effect on nymphal body weight (and therefore nymphal survival and consequently transmission from tick to rodent). 


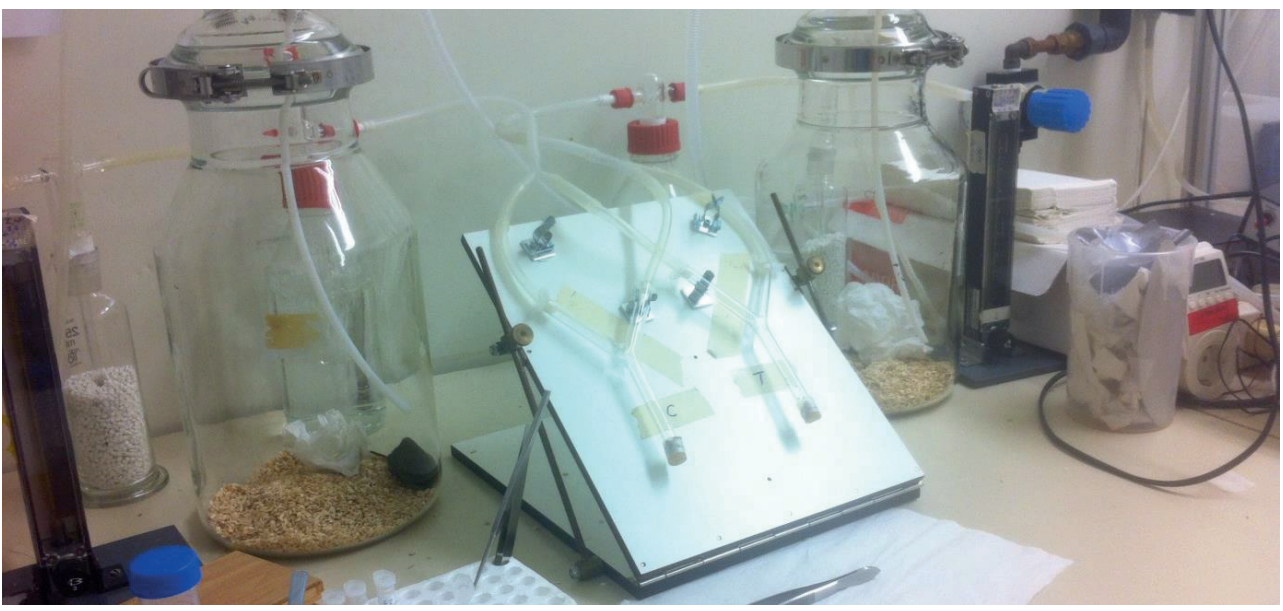

Figure 5. Experimental setup to determine the effect of a Borrelia afzelii infection in rodents on host preference of Ixodes ricinus nymphs, described in Chapter 5.

Rodents are used as host by I. ricinus larvae. The contribution of a rodent to the tick and $B$. afzelii populations does not only depend on the number of ticks feeding on it, but also on the physiology of the ticks that fed on this rodent. In Chapter 6, I first review the current knowledge on the suitability of rodents as hosts for $I$. ricinus and $B$. afzelii and then present the results of a field experiment in which the effect of rodent species on larval blood meal size and nymphal body weight was analysed. Rodents were trapped in the field and transported to the laboratory (Figure 6) so the attached ticks could complete their blood meal and drop off before the rodents were released again. Body weight of engorged larvae and emerged nymphs was determined.

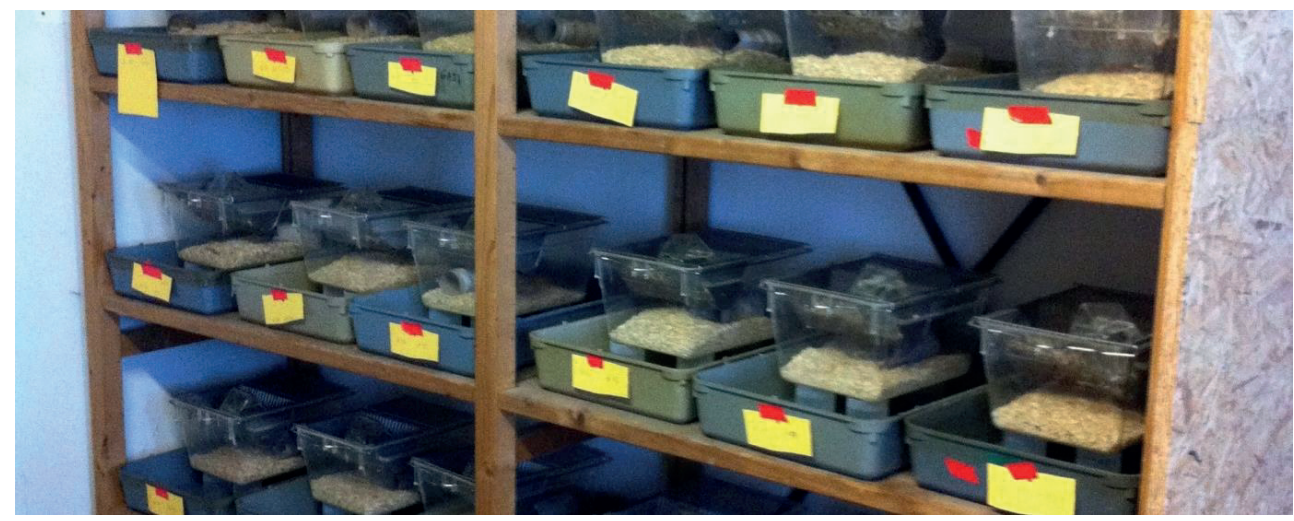

Figure 6. Individually housed field-collected bank voles and wood mice for the collection of engorged Ixodes ricinus larvae, described in Chapter 6. 
Ixodes ricinus quests in the vegetation until a host is encountered. During questing, they sense $\mathrm{CO}_{2}$ and host odour to detect their host. In Chapter 7, I study the differential effect of $\mathrm{CO}_{2}$ and host odour on the host seeking behaviour of I. ricinus. The effect of $\mathrm{CO}_{2}$ and host odour on the activation and attraction of $I$. ricinus nymphs was analysed in a Y-tube olfactometer in the laboratory as was used in experiment 5 (Figure 5). Nymphal response and preference were measured.

Rodents are born free of $B$. afzelii and it is generally assumed that they acquire the infection through the bite of an infected nymph. In Chapter 8, I investigated whether larvae of $I$. ricinus could transmit $B$. afzelii and $B$. miyamotoi to vertebrate hosts. I collected 28,000 questing larvae from five different field locations and placed them in batches on 20 laboratory mice (Figure 7). After the experiment, the mice and nymphs emerged from the engorged larvae were analysed for infection by culture and PCR.

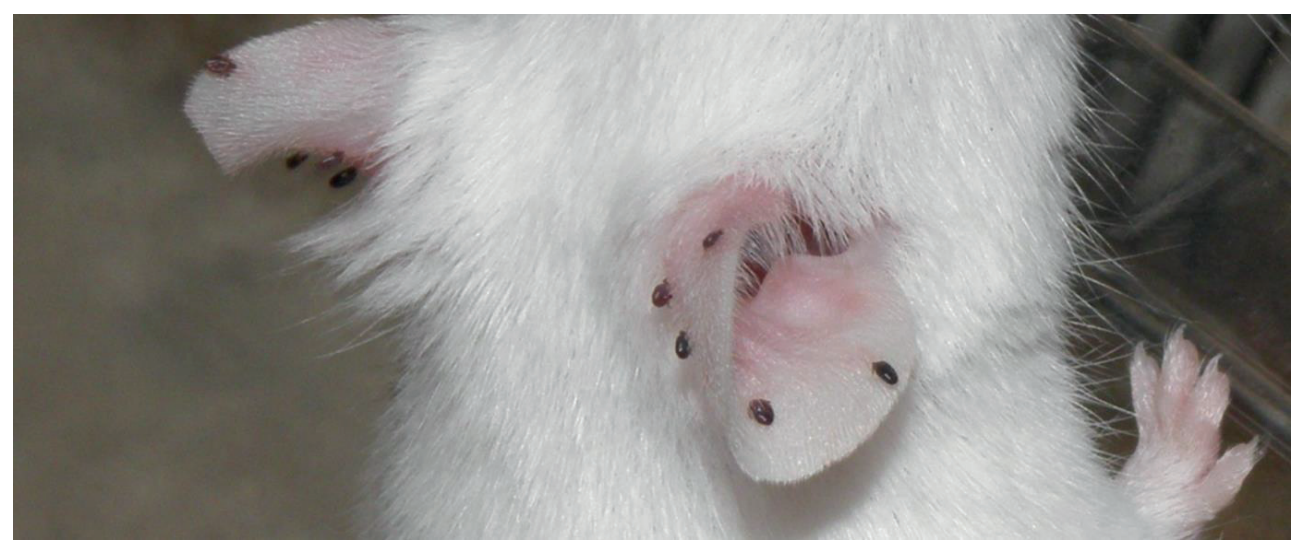

Figure 7. Field-collected Ixodes ricinus larvae feeding on the ears of a laboratory mouse to investigate whether larvae can transmit Borrelia afzelii and B. miyamotoi to rodents, described in Chapter 8.

Finally, in Chapter 9, I summarize and discuss the main conclusions of my thesis and end with suggestions for future research. 



\section{Chapter 2}

\section{Multi-trophic interactions driving the}

transmission cycle of Borrelia afzelii between Ixodes ricinus and rodents: A review

Gilian van Duijvendijk, Hein Sprong, Willem Takken 


\section{Abstract}

The tick Ixodes ricinus is the main vector of the spirochaete Borrelia burgdorferi sensu lato, the causal agent of Lyme borreliosis, in the western Palearctic. Rodents are the reservoir host of $B$. afzelii, which can be transmitted to I. ricinus larvae during a blood meal. The infected engorged larvae moult into infected nymphs, which can transmit the spirochaetes to rodents and humans. Interestingly, even though only about $1 \%$ of the larvae develop into a borreliaeinfected nymph, the enzootic borreliae lifecycle can persist. The development from larva to infected nymph is a key aspect in this lifecycle, influencing the density of infected nymphs and thereby Lyme borreliosis risk. The density of infected nymphs varies temporally and geographically and is influenced by multi-trophic (tick-host-borreliae) interactions. For example, blood feeding success of ticks and spirochaete transmission success differs between rodent species and host-finding success appears to be affected by a $B$. afzelii infection in both the rodent and the tick. In this paper, we review the major interactions between I. ricinus, rodents and $B$. afzelii that influence this development, with the aim to elucidate the critical factors that determine the epidemiological risk of Lyme borreliosis. The effects of the tick, rodent and $B$. afzelii on larval host finding, larval blood feeding, spirochaete transmission from rodent to larva and development from larva to nymph are discussed. Nymphal host finding, nymphal blood feeding and spirochaete transmission from nymph to rodent are the final steps to complete the enzootic $B$. afzelii lifecycle and are included in the review. It is concluded that rodent density, rodent infection prevalence, and tick burden are the major factors affecting the development from larva to infected nymph and that these interact to each other. We suggest that the $B$. afzelii lifecycle is dependent on the aggregation of ticks among rodents, which is manipulated by the pathogen itself. Better understanding of the processes involved in the development and aggregation of ticks results in more precise estimates of the density of infected nymphs, and hence predictions of Lyme borreliosis risk. 


\section{Introduction}

Borrelia burgdorferi sensu lato (s.I.), a tick-borne pathogen, can cause Lyme borreliosis in humans (Medlock et al. 2013). Borrelia burgdorferi s.l. consists of several genospecies, of which B. afzelii, B. garinii and B. burgdorferi sensu stricto (s.s.) are the main cause of Lyme borreliosis in the western Palearctic (Gray 1998, Grubhoffer et al. 2005). Each of these genospecies is associated with different enzootic lifecycles (Kurtenbach et al. 1998b) and clinical manifestations (Nau et al. 2009). Borrelia afzelii has been mostly associated with skin manifestations, whereas $B$. garinii is considered to be the most neurotropic and $B$. burgdorferi s.s. seems to be the most arthritogenic (Strle and Stanek 2009, Stanek et al. 2012). Depending on the geographical location, the most common genospecies in I. ricinus are $B$. afzelii and $B$. garinii (Rauter and Hartung 2005, Cadenas et al. 2007, Gassner et al. 2011, Wilhelmsson et al. 2013, Tappe et al. 2014). These genospecies are associated with different vertebrate host species. Borrelia afzelii is associated with rodents (Kurtenbach et al. 1998b, Humair et al. 1999, Hanincová et al. 2003a, Kybicova et al. 2008), whereas B. garinii is associated with birds (Kurtenbach et al. 1998b, Hanincová et al. 2003b). Because there is sufficient data on the interactions between rodents, ticks and borreliae (in contradiction to the data on birds) and because rodents are the main blood host for larvae (Matuschka et al. 1991), this review focusses on $B$. afzelii and rodents.

Ixodes ricinus is the principal vector of borreliae in the western Palearctic. This tick has three blood-feeding stages (larva, nymph and adult), which take a single blood meal before moulting to the next stage or laying eggs in the case of an adult female. Adult males do not feed. Larvae can become infected with $B$. afzelii via a blood meal from an infected rodent or via a blood meal from an uninfected host when feeding in close vicinity of a $B$. afzelii-infected tick, a cofeeding infection (Gern and Rais 1996, Randolph et al. 1996, Randolph 2011, Voordouw 2015). Rodents can become infected through the bite of an infected tick. It is generally believed that nymphs are responsible for infecting rodents because larvae are rarely infected and adults rarely feed on rodents. Nymphs are also the principle vectors that transmit borreliae to humans (Matuschka et al. 1992b). Therefore, the density of infected nymphs affects Lyme borreliosis risk, as was shown in the Nearctic (Stafford III et al. 1998). The density of infected nymphs is determined by the density of nymphs * nymphal infection prevalence.

The interactions between ticks and rodents are complex and can influence pathogen transmission (Ribeiro 1989, Randolph and Nuttall 1994). The development from uninfected larva to infected nymph is a key aspect in the enzootic borreliae lifecycle. Density of larvae is about one order of magnitude higher than the density of nymphs (Barandika et al. 2006, Barandika et al. 2010). Nymphal infection prevalence varies temporally and geographically, due to differences in climatic conditions (Estrada-Pena et al. 2011), but is about 10\% (Rauter 
and Hartung 2005, Gassner et al. 2011, Coipan et al. 2013b). As a result, only about $1 \%$ of the I. ricinus larvae develop into a borreliae-infected nymph.

The aim of this review is to give an overview of the major multi-trophic (tick-rodent- $B$. afzelii) interactions that influence the development from an uninfected larva to an infected nymph. This development depends on the success of 1) host attachment of larvae, 2) blood feeding of larvae, 3) borreliae transmission from rodent to larvae, and 4) development from engorged larva to nymph (Figure 1). Host attachment of nymphs, blood feeding of nymphs and borreliae transmission from nymph to rodent are the final steps to complete the enzootic $B$. afzelii lifecycle and therefore included. The review summarizes the current state of knowledge of the interactions between sub-adult $I$. ricinus, rodents and $B$. afzelii in the western Palearctic and how these interactions affect Lyme borreliosis risk.

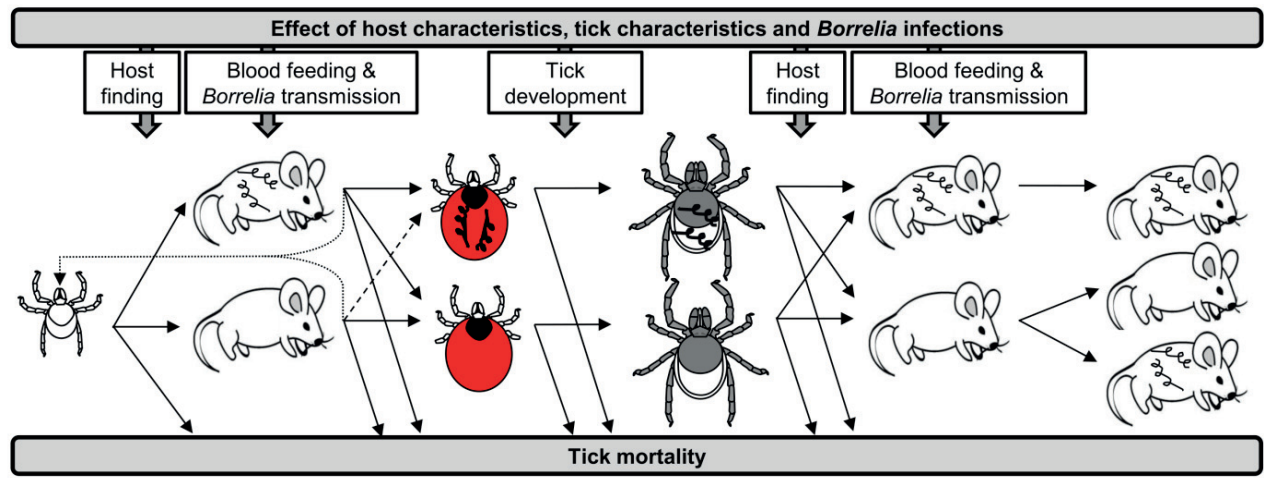

Figure 1. From larva to infected nymph. Schematic overview of the steps involved in the development from an uninfected Ixodes ricinus larva to a $B$. afzelii-infected nymph and the transmission process of $B$. afzelii between rodent and tick. Dotted lines indicates continuation of questing after a partial blood meal, dashed line indicates cofeeding transmission.

\section{Host attachment}

Ixodes ricinus feeds on a variety of host species. Each instar of the tick climbs into the vegetation and enters a host-finding stage, termed questing, and senses hosts by $\mathrm{CO}_{2}$, host volatiles and vibrations (Lees 1948, Osterkamp et al. 1999, Gherman et al. 2012). Questing height was lower for larvae compared to nymphs (Mejlon and Jaenson 1997). During questing, water is lost from the tick, which can be reabsorbed when in the litter layer (Lees 1946). Attachment to a host is the first major step in the development from larva to nymph, but the chance that a larva encounters a rodent is unknown. Instead, tick burden will be used as a measurement of host-attachment success. Tick burden is determined by tick encounter rate, attachment success, grooming and tick feeding duration (Mannelli et al. 2012). Factors 
affecting larval and nymphal host attachment are comparable and therefore combined in this paragraph.

\section{Host effects}

The chance that a questing larva encounters a host affects the density of nymphs and is influenced by the density and activity of hosts (Jones et al. 1998, Rosa et al. 2007), which vary between host species. Tick burdens vary between the most common rodent species in Europe; wood mouse (Apodemus sylvaticus), yellow-necked mouse (Apodemus flavicollis) and bank vole (Myodes glareolus). Larval tick burden is higher on wood mice than on bank voles (Nilsson and Lundqvist 1978, Humair et al. 1993, Kurtenbach et al. 1995, Tälleklint and Jaenson 1997, Gray et al. 1999, Boyard et al. 2008, Gassner et al. 2013) which may be caused by differences in ecological niche, activity, home range, grooming activity and immune response (Kaufman 1989, Wikel 1996, Hughes and Randolph 2001). Bank voles have an innate and acquired tick resistance resulting in a lower tick attachment success compared to wood mice (Randolph 1979, Kaufman 1989, Randolph 1994, Dizij and Kurtenbach 1995, Wikel 1996, 1999, Hughes and Randolph 2001). Ixodes ricinus can sense their host by smell (Berret and Voordouw 2015) and may even be able to distinguish between host species as was shown in the Nearctic for $I$. scapularis (James and Oliver Jr 1990, Shaw et al. 2003). The genetic population structure of I. ricinus indicated that the species shows some host specialization (Kempf et al. 2011). The scale of this specialization is, however, unknown. Tick burden also varies within host species. In general there are many individuals with low tick burdens and few hosts with high tick burdens, feeding the majority of ticks (Tälleklint and Jaenson 1997, Heylen et al. 2013c), following the 20/80 rule (Woolhouse et al. 1997, Perkins et al. 2003). This intra-species variation can be influenced by sex, age, body mass and activity of the host. In general, tick burden is higher on males compared to females, older rodents compared to younger rodents, heavy weight rodents compared to light weight rodents and active rodents compared to lessactive rodents (Sonenshine and Stout 1968, De Boer et al. 1993b, Craine et al. 1995, Tälleklint and Jaenson 1997, Harrison et al. 2010, Pisanu et al. 2010, Taylor et al. 2013b). However, these relationships are complex, can be correlated to each other (e.g. males have a greater body weight than females) or interact with each other (tick burdens on females decreased with age whereas they increased on males) (Brunner and Ostfeld 2008, Heylen et al. 2013c).

Host preference of $I$. ricinus has not been tested experimentally, but there are examples of other tick species that show an intra-species host preference. Dermacentor variabilis preferred the odours from larger and male mice over smaller and female mice (Dallas and Fore 2013), I. arboricola preferred well developed bird nestlings over less developed nestlings (Heylen and Matthysen 2011), whereas I. hexagonus preferred sick hedgehogs over healthy ones (Bunnell 
et al. 2011). Testosterone can also affect tick burden. It facilitates dominance in wild mice (Zielinski and Vandenbergh 1993) and reduces innate and acquired resistance to ticks (Hughes and Randolph 2001). Testosterone levels also differed between rodent species and the level was 10 times higher in wood mice compared to bank voles (Hughes and Randolph 2001). High testosterone levels can also reduce tick feeding speed, as was found for ticks feeding on lizards (Pollock et al. 2012).

\section{Tick effects}

Ectoparasites such as ticks affect the fitness of their hosts in various ways. For example, only $0.17 \%$ blood loss of gerbils (Gerbillus dasyurus) resulted in a $16 \%$ increased energy use (Khokhlova et al. 2002). This increased energy use should be compensated by an increased energy uptake and therefore host activity, increasing ectoparasite encounter rate. Feeding ticks can consume up to $65 \%$ of the blood from a rodent (Tälleklint and Jaenson 1997), affecting fitness and activity. Tick feeding can also cause erosion of the ear margin (Harris et al. 2009), reducing host fitness. Hosts with a larger home range have a higher reproductive success but may also have a higher tick burden, as was shown for $I$. scapularis and $D$. variabilis (Sonenshine and Stout 1968, Devevey and Brisson 2012). Larval tick burdens on rodents increased with increasing densities of questing larvae, but it was suggested that rodents can become saturated with larvae (Brunner and Ostfeld 2008). In addition, the heterogeneity in larval tick burdens on rodents can also be affected by the heterogeneous dispersal of larvae in the environment, increasing the chance of acquiring multiple larvae simultaneously.

\section{Borreliae pathogen effects}

There is abundant evidence that pathogens can influence their host and/or vector to enhance transmission (Moore 1995, Lefèvre and Thomas 2008). Evidence of borreliae manipulating host attachment of ticks is, however, scarce. In the field, borreliae-infected rodents have higher tick burdens compared to borreliae-uninfected rodents (Hanincová et al. 2003a, Gassner et al. 2013, Taylor et al. 2013b). Once infected, spirochaete load did not affect tick burden on rodents (Raberg 2012). It is unclear whether borreliae can manipulate tick burden (e.g. due to a higher energy demand or altered odour of the rodent) or whether a high tick burden increased exposure risk to borreliae. Hosts with high nymphal tick burdens have a higher chance of becoming infected with borreliae and rodents infested with nymphs have higher larval tick burdens than rodents without nymphs (Craine et al. 1995, Tälleklint and Jaenson 1997, Bown et al. 2008). A borreliae infection does not affect rodent survival (Radolf et al. 2012, Voordouw et al. 2015), but a specific borreliae antibody response altered foraging behaviour of white 
footed mice in the Nearctic (Schwanz et al. 2011b, Schwanz et al. 2012), which may increase tick encounter rate. There is also evidence that borreliae can influence host-tick contact rate when in the tick. A borreliae infection in adult female I. ricinus increased host finding efficacy (Faulde and Robbins 2008). In addition, borreliae-infected nymphs had higher energy reserves and spent more time questing for a host compared to borreliae-uninfected nymphs (Mejlon and Jaenson 1997, Gassner 2010, Herrmann and Gern 2010, 2012, Herrmann et al. 2013b, Herrmann and Gern 2015). These effects were influenced by B. burgdorferi s.I. genospecies (Herrmann and Gern 2010). However, all these results are from field-collected ticks and observed differences may, therefore, have been caused by characteristics of the host on which the ticks fed as larvae (e.g. species, tick burden, immune response). If, for example, tick burden positively affects blood meal size (see above), borreliae-infected ticks will have a higher fat content due to the higher tick burden of infected rodents, while this was not caused by the borreliae infection of the tick.

\section{Blood feeding}

Once a sub-adult tick has encountered a rodent, it needs to find a feeding site, bite the host and acquire a blood meal. It is generally assumed that each larva takes only one blood meal before moulting to a nymph. Factors affecting larval and nymphal blood feeding are comparable and therefore combined in this paragraph.

\section{Host effects}

Blood feeding of $I$. ricinus is a complex process with major events occurring within the tick (1989) and can be influenced by host species. Blood meal size and percentage of fully engorged ticks are larger for larvae feeding on Apodemus mice, which therefore moult into larger nymphs, compared to larvae that fed on bank voles (Nilsson and Lundqvist 1978, Matuschka et al. 1992a, Tälleklint and Jaenson 1997, Hughes and Randolph 2001). Exposure to tick saliva caused acquired tick resistance in bank voles, resulting in a decreased blood ingestion speed (Randolph 1979, 1994, Dizij and Kurtenbach 1995, Hughes and Randolph 2001), whereas this was increased in yellow necked mice (Dizij and Kurtenbach 1995). In addition, feeding duration had a positive effect on blood meal size in bank voles, but not in wood mice (Nilsson and Lundqvist 1978). The effect of acquired tick resistance on tick feeding in I. ricinus was also found for rabbits (Alani and Herbert 1987). 


\section{Tick effects}

Tick saliva has anti-haemostatic, anti-inflammatory, and immunosuppressive effects on the host's immune system, facilitating blood consumption of ticks (Wikel 1999, Gillespie et al. 2000). A larger tick burden results in more tick saliva and a higher immunosuppressive effect, which could therefore facilitate blood feeding of ticks. As a result, a high tick burden increased feeding success of $I$. ricinus feeding on wood mice and bank voles (Hughes and Randolph 2001).

\section{Borreliae pathogen effects}

There are indications that a borreliae-infection results in an increased blood meal size of larvae. Infected engorged larvae collected from wood mice were heavier and moulted into larger nymphs compared to uninfected larvae (Gassner 2010). The higher energy reserve of borreliae-infected nymphs (see above) is also likely to be a result of a larger blood meal during the larval stage. However, these differences could have been caused by a higher tick burden of infected hosts, affecting the immune response of the host (see above). In an artificial feeding system, blood meal size of nymphs decreased when fed Bartonella-infected blood compared to Bartonella-uninfected blood, whereas feeding duration was not affected (Liu et al. 2014).

\section{Development from engorged larva to nymph}

A larva that acquired a complete blood meal detaches from the host to digest their blood meal and moult into a nymph. When the larva acquired borreliae during this blood meal, it will emerge as an infected nymph after moulting.

\section{Host effects}

Moulting success from larva to nymph can be influenced by host species and was higher for larvae that naturally attached to field collected Apodemus spp. compared to bank voles (Humair et al. 1999, Burri et al. 2014), but the opposite happened for laboratory reared ticks (Burri et al. 2014). After multiple infestations, moulting success remained stable in Apodemus mice but declined in bank voles; this effect was abolished, however, when testosterone levels were increased (Dizij and Kurtenbach 1995, Hughes and Randolph 2001). A reduced moulting success was also found for $I$. trianguliceps feeding on bank voles (Randolph 1994) and may have been caused by a difference in blood meal success (see above) because partiallyengorged larvae failed to moult (Dizij and Kurtenbach 1995). 


\section{Tick effects}

Endosymbionts are widespread among arthropods (Dennison et al. 2014, Zug and Hammerstein 2015). The effects of endosymbionts have not been investigated in $I$. ricinus. However, in the Nearctic they have been shown to influence tick fitness (Zhong et al. 2007) and the colonization of borreliae in the tick (Narasimhan et al. 2014). The relationship between the tick microbiome and tick survival and borreliae transmission are far from understood (Narasimhan and Fikrig 2015) and has not been investigated in I. ricinus.

\section{Borreliae pathogen effects}

During moulting, borreliae spirochaetes survive in the midgut lumen of the tick and persistence until the next feeding is crucial for successful transmission (Burgdorfer et al. 1982, Burgdorfer 1984). The interactions between the tick's defence mechanisms and borreliae during moulting have been reviewed (2013). It was shown that in the case of I. scapularis, even though borreliae load is reduced fivefold during moulting and remained stable at $<300$ spirochaetes in the emerged nymph (Piesman et al. 1990), spirochaete genetic population structure was not affected during moulting (Rego et al. 2014). Whether a borreliae infection affects interstadial development from $I$. ricinus larva to nymph is unknown.

\section{Borrelia transmission from rodent to larva}

To maintain the enzootic borreliae lifecycle, rodents must feed both larvae and nymphs and an infection acquired by a larva must be transstadially transmitted during the moult to a nymph. Feeding larvae can also become borreliae-infected through co-feeding with an infected nymph on a host without a systemic infection (Gern and Rais 1996, Randolph et al. 1996, Randolph 2011). However, because rodents are the main host used by larvae and can be systemically infected with borreliae, the effect of co-feeding transmission on the zoonotic life cycle of borreliae appears to be limited. The chance that a larva acquires borreliae from a host is determined by the borreliae prevalence in the host community, which is influenced by the probability that infected nymphs feed on the host, host susceptibility to the pathogen and the ability of the host to maintain the infection. The survival of borreliae within the host and tick, and transmission between them, are underpinned by molecular mechanisms, which have been reviewed (Schuijt et al. 2011, Mannelli et al. 2012). 


\section{Host effects}

Not all host species used by $I$. ricinus are borreliae reservoirs and there is high variation in transmission efficiency among reservoir hosts. Rodents are associated with $B$. afzelii (Kurtenbach et al. 1998b, Humair et al. 1999, Hanincová et al. 2003a, Kybicova et al. 2008). Borrelia burgdorferi sensu stricto, B. bavariensis and B. spielmanii are also associated with rodents, but have a lower infection prevalence in questing nymphs (Richter et al. 2004b, Margos et al. 2009, Gassner et al. 2011). Rodents can also be co-infected with multiple $B$. burgdorferi s.l. genospecies (Zore et al. 1999, Kybicova et al. 2008, Schmidt et al. 2014a). However, these different genospecies were not necessarily acquired through the bite of one nymph co-infected with multiple genospecies, but could be transmitted by multiple infected nymphs. Large mammals like roe deer and red deer are hosts for ticks, but incompetent for borreliae transmission, presumably because of anti-borreliae immune responses (Jaenson and Talleklint 1992, Pacilly et al. 2014). In the Nearctic, rodent infection rate (percentage of infected hosts) and host infectivity (percentage of uninfected larvae that acquire a borreliae infection during feeding on an infected host) are positively correlated and vary between host species (Brunner et al. 2008). Whether this is also true for the western Palearctic is not known. Rodent infection rate is lower in wood mice compared to bank voles and varies temporally and geographically (Tälleklint and Jaenson 1994, Humair et al. 1999, Kybicova et al. 2008, Gassner et al. 2013). Rodent infection rate can also differ between sexes and was higher in males compared to females (Tschirren et al. 2013), which was likely due to higher nymphal tick burdens on males, increasing exposure to borreliae. Infected rodents stay infective throughout their life resulting in a higher rodent infection rate of older rodents compared to younger rodents (Gern et al. 1994). Borreliae infection prevalence of ticks fed on wild rodents was lower in April (1.2-10.5\%) compared to June/July (15.1-17.5\%) and did not increase until October (Kurtenbach et al. 1995), which is probably explained by a lower rodent infection rate caused by a lower exposure to borreliae-infected nymphs during winter compared to spring, summer and autumn.

Host infectivity is also influenced by host species and is lower in mice compared to voles (Kurtenbach et al. 1995, Humair et al. 1999, Perez et al. 2012, Radzijevskaja et al. 2013). The differences between wood mice and bank voles can be caused by the number of borreliaespecific antibodies in the host, which correlated negatively to infectivity (Kurtenbach et al. 1994). Even though infections were not lost, host infectivity can vary over time and decreased with increasing time since infection rodents (Richter et al. 2004a, Tschirren et al. 2013). A correlation between host body size versus infectivity and spirochaete burden in feeding ticks has not been tested for $I$. ricinus. However, this correlation was negative at host species level 
for I. scapularis (Barbour et al. 2015). These authors suggested that this was caused by a difference in time between inoculation and putative threshold for infectiousness.

\section{Tick effects}

The aggregation of ticks among hosts results in an increased borreliae transmission when larval and nymphal tick burdens are correlated. In addition, infectivity increased with successive larval infestations and larval tick burden (Gern et al. 1994, Kurtenbach et al. 1995), increasing the contribution of these heavily infested individuals. Host infectivity of host associated B. burgdorferi s.I. -genospecies (B. garinii and B. valaisiana for birds) increased with successive infestations with field collected I. ricinus nymphs, whereas infectivity of genospecies associated with other hosts ( $B$. afzelii for birds) decreased, suggesting a possible developed resistance (Heylen et al. 2013a). The effect of tick burden on infectivity may be caused by the immunosuppressive effect of tick saliva on the rodent immune system (Wikel 1999, Gillespie et al. 2000), resulting in an increased infectivity. However, infectivity of bank voles was reduced at sites with high tick densities (Kurtenbach et al. 1995). Borreliae transmission from host to ticks increases with feeding time and started 2-8 $\mathrm{h}$ after tick attachment (Richter et al. 2012). Borrelia afzelii has to survive the tick immune system during blood digestion, moulting and migration via the haemolymph to the salivary glands (Johns et al. 2001). Nymphal infection prevalence had a positive effect on infection prevalence of larvae fed on rodents (Kurtenbach et al. 1995), most likely due to a higher exposure of rodents to infected nymphs.

\section{Borreliae pathogen effects}

Rodent infection rate varies between $B$. burgdorferi s.l. genospecies and is highest for $B$. afzelii, followed by B. burgdorferi s.s. and B. garinii (Kybicova et al. 2008, Gassner et al. 2013). Hosts can transmit multiple genospecies to feeding ticks (Heylen et al. 2013a), but host infection does not necessarily mean that the spirochaetes are transmitted to feeding ticks, as was shown for rodents infected with $B$. garinii in internal organs, which only transmitted $B$. burgdorferi s.s. to feeding larvae (Kurtenbach et al. 1998b). Spirochaete load of $B$. burgdorferi s.s. was higher when mice were co-infected with $B$. garinii, compared to an infection with only B. burgdorferi s.s., whereas the opposite happened for B. garinii (Hovius et al. 2007), indicating interactions between the two genospecies while in the same host, which benefits $B$. burgdorferi s.s. Time until infectiousness also differs between genospecies; wood mice became infectious with $B$. afzelii in fewer days post infection and with a higher infectivity compared to $B$. burgdorferi s.s. (Richter et al. 2004a, Radolf et al. 2012). Borrelia burgdorferi s.s. was found 
only in rodents during tick activity, but not during winter (Humair et al. 1999), suggesting that these reservoir hosts are not a permanent reservoir for all genospecies and can lose infections, as was shown in the Nearctic for Peromyscus leucopus (Lindsay et al. 1997). However, B. burgdorferi s.s. was also only found in mouse blood up to eight days after inoculation, whereas spirochaetemia lasted up to six weeks after inoculation (Hovius et al. 2007). Infectivity also differs between borreliae isolates, as was shown for B. afzelii (Tonetti et al. 2015). The increased host's infectivity with time (see above) was also genospecies dependent and increased faster in B. afzelii compared to B. burgdorferi s.s. (Richter et al. 2012). Borrelia afzelii (rodent associated) on the one hand and B. garinii and B. valaisiana (bird associated) on the other hand infect adult $I$. ricinus on a mutualistic exclusive way; they co-occurred less frequently than expected compared to co-infections with $B$. garinii and $B$. valaisiana (Kurtenbach et al. 2002c). Whether these different genospecies were transmitted during a single feed on one host or two feeds on separate hosts (as larva and nymph) is unclear, but it seems likely that $B$. afzelii in nymphs feeding on birds was negatively selected by host complement in the midgut of feeding ticks (Kurtenbach et al. 1998a). Strong genetic differentiation was observed between $B$. burgdorferi s.l. genotypes infecting different rodent species, suggesting host specificity of borreliae populations (Jacquot et al. 2014). Spirochaete load at the feeding site positively influenced host infectivity (Raberg 2012, Rego et al. 2014) and rodents with a high infectivity transmit more borreliae spirochaetes to larvae compared to the larvae fed on rodents with a lower infectivity (Gern et al. 1994). However, even though spirochaete load was ten times higher in voles compared to mice, this did not result in a higher infectivity of voles compared to mice and this was probably due to a larger blood meal size on mice (Raberg 2012). If a high spirochaete load in rodents results in a high spirochaete load in feeding ticks, infectivity from tick to host may also be enhanced. Spirochaete load in rodents and feeding ticks were, however, not correlated (Raberg 2012).

\section{Borrelia transmission from nymph to rodent}

Rodents acquire a borreliae infection through the bite of an infected tick and not via vertical transmission from female to offspring, as was shown for the Nearctic reservoir host Peromyscus leucopus (Wright and Nielsen 1990, Mather et al. 1991). Ixodes ricinus larvae are rarely infected with $B$. burgdorferi s.l. (Richter et al. 2012) and adults rarely feed on rodents (Gassner et al. 2013), suggesting that nymphs are responsible for transmitting borreliae to rodents. After a borreliae-infected nymph attaches to a host, the borreliae spirochaetes in the midgut multiply and migrate through the midgut wall via the haemolymph to the salivary glands, from which they may be inoculated with the tick saliva into the host (Gern 1990, Gern et al. 1996). Borreliae transmission from nymph to host is positively correlated with feeding duration 
of the tick and in general does not occur before $24 \mathrm{~h}$ of feeding (Crippa et al. 2002, Cook 2015). However, borreliae can be transmitted as early as after 16-17 h of feeding (Kahl et al. 1998), which may have been caused by a systemic borreliae infection in the tick (Leuba-Garcia et al. 1994). Once spirochaetes have been inoculated into the host's skin, they remain at the inoculation site and disseminate after a few days, as was shown for B. burgdorferi s.s. in the Nearctic (Shih et al. 1992). Borreliae have been detected in skin, blood, joints, spleen, heart, liver, urinary bladder, kidney and nervous system of vertebrate hosts (Snydman et al. 1986, Callister et al. 1989, Sinsky and Piesman 1989, Hofmeister et al. 1992).

\section{Host effects}

Not all host species are susceptible to each B. burgdorferi s.l. genospecies, due to differences in complement-mediated sensitivity of the spirochaetes to host serum (Kurtenbach et al. 1998a). Borrelia afzelii is mainly associated with rodents (Kurtenbach et al. 1998b, Hanincová et al. 2003a), B. garinii and B. valaisiana with birds (Kurtenbach et al. 1998b, Hanincová et al. 2003b), B. lusitaniae with lizards (Richter and Matuschka 2006) and B. spielmanii with dormice (Richter et al. 2011). As a result, an infected tick that feeds on a host that is incompetent for the concerning genospecies appears to lose its infection (Matuschka and Spielman 1992, Kurtenbach et al. 2002b, Pacilly et al. 2014). However, this is not always the case, as was shown for $B$. afzelii in songbirds (Heylen et al. 2013a). In the Nearctic, pre-exposure of rodents to $I$. scapularis reduced susceptibility to borreliae, irrespective of an acquired tick immunity (Wikel et al. 1997, Nazario et al. 1998). This suggests that nymphal infection prevalence can influence rodent infection rate directly (a low nymphal infection prevalence reduces borreliae exposure to the host) or indirectly (a low nymphal infection prevalence reduces host susceptibility to borreliae) and that a high larval tick burden may reduce rodent infection rate by acquired immunity. This has not been investigated for $I$. ricinus, but indeed, rodent infection rate of white footed mice was ten times higher in periods with high risk of exposure to $I$. scapularis nymphs compared to a period of low risk (Hofmeister et al. 1999). Susceptibility to borreliae differed between bank vole individuals and was influenced by their genetic variation (Tschirren et al. 2013).

\section{Tick effects}

When Borrelia-infected $I$. ricinus nymphs can feed to repletion, transmission success from nymph to host was almost $100 \%$ (Kahl et al. 1998). Nymphs do not need to have acquired the borreliae spirochaete(s) during a blood meal in the larval stage. It was shown for I. scapularis that nymphs can acquire borreliae during an interrupted feeding of $16 \mathrm{~h}$ and can infect another 
host after 3-5 days without first moulting to the next stage (Shih and Spielman 1993). However, larvae that fed partially (18 h) on a borreliae-infected host were not infectious during a second blood meal five weeks after the initial feeding, whereas borreliae were transmitted after they moulted into nymphs (Piesman 1991). Partially-fed ticks can arise by tick immunity of the host (Dizij and Kurtenbach 1995). In addition, grooming of the host or host mortality may also result in partially-fed ticks. However, whether this can also happen in I. ricinus and frequencies of naturally occurring partially-fed larvae and nymphs are unknown.

\section{Borreliae pathogen effects}

Ticks can be co-infected with more than one B. burgdorferi s.I. genospecies (Leuba-Garcia et al. 1994, Pichon et al. 1995, Rauter and Hartung 2005, Gassner et al. 2011, Wilhelmsson et al. 2013), with up to $45 \%$ of infected ticks harbouring multiple genospecies (Misonne et al. 1998). Even though adult ticks have taken an additional blood meal, co-infection prevalence was not higher in adults compared to nymphs (Rauter and Hartung 2005), which may be caused by the clearance of the genospecies acquired during the first blood meal by the ingestion of host complement during the second blood meal. Therefore, at least in the case of the nymphal stage, co-infections are likely to be acquired during one single blood meal from a co-infected host. The majority of co-infected nymphs are therefore co-infected with two genospecies that can co-occur in the same host (Misonne et al. 1998, Herrmann et al. 2013a). Spirochaete load in nymphs co-infected with genospecies that share vertebrate hosts was equal to or higher than the additive expectation, whereas this was lower for genospecies associated with different reservoir hosts (Herrmann et al. 2013a). Spirochaete load in infected ticks was higher for B. garinii and B. bavariensis compared to B. afzelii (Herrmann and Gern 2012, Herrmann et al. 2013a). Even though all spirochaete clones present in the host were transmitted to the feeding larvae and survived moulting to the nymphal stage, only a small fraction of the spirochaetes in the tick's midgut are transmitted from nymph to host during feeding (Dunham-Ems et al. 2009, Rego et al. 2014). Whether a high spirochaete load in infected nymphs results in a greater transmission success to a host during feeding is unknown. When injected intradermally, only 10 cultured borreliae spirochaetes were enough to infect a mouse (Barthold 1991).

\section{Conclusions}

Understanding the factors that affect the density of infected nymphs increases our knowledge on Lyme borreliosis risk. The development from questing I. ricinus larva to borreliae-infected nymph is affected by many biological and ecological factors. The existence of different $B$. 
burgdorferi s.l. genospecies and heterogeneity between and within genospecies makes the tick-rodent-borreliae interactions complex. The development from larva to nymph, regardless of a borreliae infection, affects nymphal density and appears to be successful in only $10 \%$ of the time (Randolph and Craine 1995). The chance that a larva encounters a rodent affects the density of nymphs and is influenced by rodent density, which differs between rodent species and varies spatially and temporally (De Boer et al. 1993b, Kurtenbach et al. 1995). Even though it is a major step in the development, there is no data of the chance that a larva actually encounters a rodent or any other host. Host encounter rate may not be fully dependent on external factors but may be affected by the tick too, e.g. when larvae are attracted to a rodent trail. Nymphs for example, are attracted to perches that have been scented with rodent odour (Berret and Voordouw 2015). It was shown that ticks prefer odours from certain hosts over others. However, it is unknown if questing larvae can afford to reject a non-preferred host, risking the possibility of not acquiring any blood meal and starving to death.

Rodents with high larval tick burdens, which are major contributors to the density of nymphs, have in general also higher nymphal tick burdens, making them more likely to be infected with borreliae. As a result, these rodents are even larger contributors to the density of infected nymphs. We hypothesize that this aggregation increases nymphal infection prevalence and that this aggregation is therefore necessary for the maintenance of the enzootic borreliae lifecycle. There is some evidence that the aggregation of ticks can be caused by borreliae (Hanincová et al. 2003a, Gassner et al. 2013). Therefore, the chance that a larva acquires a blood meal from a Borrelia-infected rodent may not solely be the effect of the density of infected rodents, e.g. when borreliae-infected rodents are more active than uninfected rodents, or when questing larvae prefer - the odours from - borreliae-infected rodents, the chance of acquiring a borreliae infection is greater than the effect of rodent density alone. Even though the only experimental study conducted on this subject showed no effect of a borreliae infection in rodents on tick attraction (Berret and Voordouw 2015), there are many examples of parasites manipulating their hosts (Moore 1995, Lefèvre and Thomas 2008). Therefore, understanding rodent or tick manipulation by borreliae requires more experiments with experimentally infected rodents and ticks to exclude biases from differences in rodent characteristics on physiological or behavioural differences between infected and uninfected rodents and ticks.

The borreliae lifecycle does not only benefit from aggregation of larvae on (borreliae-infected) rodents, but also from the successful development from larvae to nymph and the chance that a borreliae-infected nymph encounters a (borreliae-uninfected) rodent. Whether borreliae can affect interstadial tick development (e.g. moulting success) is unknown and requires more research too to overcome a bias in the effect of the $90 \%$ mortality during development from larva to nymph (Randolph and Craine 1995). 
Tick survival, rodent density, rodent infection rate and host infectivity are major factors affecting the borreliae lifecycle, whereas only the first two directly affect the tick lifecycle. These factors also interact with each other, e.g. even though infection rate and infectivity of bank voles was higher, the higher tick burden on wood mice and moulting success of ticks fed on wood mice made wood mice more important contributors to the density of infected nymphs (Humair et al. 1999). The tick lifecycle clearly benefits from a high density of nymphs, whereas the borreliae lifecycle benefits from a high nymphal infection prevalence. However, the borreliae lifecycle also benefits from a high density of nymphs, when this will lead to a higher density of larvae and therefore a higher chance of borreliae transmission from rodent to tick and vice versa. There is evidence that borreliae can affect tick survival, increasing the density of nymphs and therefore enhancing its own lifecycle. Therefore, in addition, the tick lifecycle also benefits from a high nymphal infection prevalence.

More knowledge on these multi-trophic interactions helps to obtain better estimates of the Lyme borreliosis risk. This review showed the various factors that contribute to the density of infected nymphs, and how they interact. These results, together with the effect of abiotic factors, could be mathematically modelled to determine the key processes that determine the density of infected nymphs, and thereby Lyme borreliosis risk.

\section{Acknowledgements}

We thank two anonymous reviewers for their comments on an earlier version of the manuscript. 



\section{Chapter 3}

\section{Rodent density affects Lyme borreliosis risk by affecting the density of Ixodes ricinus nymphs, but not their infection rate with Borrelia burgdorferi sensu lato}

Gilian van Duijvendijk, Frans Jacobs, Manoj Fonville, Gerrit Gort, Hein Sprong, Willem Takken 


\section{Abstract}

Lyme borreliosis is caused by Borrelia burgdorferi sensu lato (s.I.), a spirochaete transmitted by the tick Ixodes ricinus. Rodents are reservoir to $B$. afzelii, the most common genospecies of $B$. burgdorferi s.I., and can infect $I$. ricinus larvae, which often feed on rodents. Infected engorged larvae emerge as infected nymphs one year later. The density of infected nymphs (DIN) positively affects Lyme borreliosis risk. The aim of this study was to determine the effect of rodent density on the DIN in a natural ecosystem, and hence, Lyme borreliosis risk.

Rodent density was manipulated by either removing rodents or adding acorns to experimental plots. Control plots received no treatment. Rodent density and rodent infection rate were determined every three months for 28 months. Density of nymphs (DON) and nymphal infection prevalence (NIP) were determined monthly for 40 months.

Rodent density had a significantly positive effect on the DIN one year later $(P=0.0018)$. This effect was caused by an increase in DON, whereas NIP was not affected. The DON and DIN differed between years, whereas NIP not. Rodent density also had a positive effect on rodent infection rate and nymphal tick burden in the same year and these effects did not vary significantly between years. The effect of rodent density on larval tick burden in the same year was negative in year one and positive in years two and three.

The results underline the importance of rodents as blood host for $I$. ricinus larvae and show a contribution of rodents to the DIN one year later. Rodent density, therefore, affects Lyme borreliosis risk, providing important information for the prediction of Lyme borreliosis risk. 


\section{Introduction}

Lyme borreliosis is caused by Borrelia burgdorferi sensu lato (s.I.) and, in the northern hemisphere, its occurrence has increased in the last decades (Medlock et al. 2013). In Europe, $B$. burgdorferi s.l. is predominantly transmitted by the sheep tick, Ixodes ricinus. In The Netherlands, infectious tick bites have increased almost four times in the last two decades, reaching an estimated 13 cases per 10,000 inhabitants in 2009 (Hofhuis et al. 2015). It is suggested that this increase is caused by an increased number of infected ticks (Sprong et al. 2012). Ixodes ricinus is the most common tick species in western Europe and has three bloodfeeding stages (larva, nymph and adult). Each instar searches for a host (a behaviour called questing) and takes one blood meal before moulting to the next stage or laying eggs in the case of an adult female. Larvae of $I$. ricinus feed primarily on small hosts such as wood mice and bank voles, nymphs on rodents and birds and adults on large hosts like deer (Hofmeester et al. 2016). When a blood meal is taken form an infected rodent, ticks can become infected with the spirochaetes. Ticks can also become infected during co-feeding with an infected tick on the same host without a systemic infection in the host (Gern and Rais 1996, Randolph et al. 1996, Randolph 2011). Rodents can become infected through the bite of an infected tick. The most common B. burgdorferi s.l. genospecies in The Netherlands is $B$. afzelii, which is linked to rodents (Humair et al. 1995, Kurtenbach et al. 1998a, Humair et al. 1999, Hanincová et al. 2003a, Kybicova et al. 2008), suggesting that rodents are important hosts for larvae. The density of nymphs (DON) and nymphal infection prevalence (NIP) determine the density of infected nymphs (DIN), which affects Lyme borreliosis incidence (Stafford III et al. 1998). It is assumed that the DON is affected by the abundance of rodents and NIP by rodent infection rate. In general, surplus rodent feed (mast) increases length of the breeding season of forest rodents and population density the following year (Jensen 1982, Pucek et al. 1993, Ostfeld et al. 1996, Jones et al. 1998, McShea 2000, Clotfelter et al. 2007). Acorn availability also increases deer density (Jones et al. 1998), which can also affect tick density (Ostfeld et al. 2006) as deer serve as hosts for adult ticks. A high rodent density would result in a high host encounter rate for questing larvae and therefore, a high DON one year later. A high rodent density can, however, also reduce tick burden on rodents by dilution (Krasnov et al. 2007, Rosa et al. 2007, Kiffner et al. 2011) and as a result, rodent infection rate and, therefore, NIP. The effect of rodent density on the DIN, and hence, Lyme borreliosis risk, has never been experimentally analysed. This study, therefore, aims to unravel the effect of rodent density on the DIN. 


\section{Materials and methods}

\section{Study site}

The study was conducted at the forest reserves Planken Wambuis $\left(52^{\circ} 01^{\prime} 45^{\prime \prime} \mathrm{N}, 5^{\circ} 48^{\prime} 49^{\prime \prime} \mathrm{E}\right)$ and Noord Ginkel (52 $\left.02^{\prime} 23^{\prime \prime} \mathrm{N}, 5^{\circ} 45^{\prime} 09^{\prime \prime} \mathrm{E}\right)$ near the city of Wageningen, The Netherlands. Both forests are dominated by scotch pine and harbour a diversity of bird and mammal species, including wood mice (Apodemus sylvaticus), bank voles (Myodes glareolus), common shrews (Sorex araneus), wild boar (Sus scrofa), roe deer (Capreolus capreolus), red deer (Cervus elaphus) and free ranging cattle and horses.

\section{Climate}

Hourly measurements of temperature and relative humidity were collected from the nearest weather station (Deelen, KNMI, The Netherlands). Saturation deficit, which is a measure for the drying power of the air and negatively affects questing duration of $I$. ricinus was calculated from these variables (Perret et al. 2000, Perret et al. 2003).

\section{Manipulation of rodent density}

In both forests six plots of $50 * 50$ meter were selected with at least $350 \mathrm{~m}$ between plots. Variation in rodent density was experimentally increased by artificial manipulation of rodent populations. Per forest, plots were assigned randomly to one of three treatments (rodent removal $(R R)$, control $(C)$ or acorn addition $(A A)$ ). Rodents were removed from four plots to reduce rodent density $(R R)$. Rodents were trapped for one night with Heslinga live traps (Heslinga Traps, Groningen, The Netherlands) in a $5^{\star} 5$ grid with $10 \mathrm{~m}$ inter-trap distance. Captured rodents were euthanized by cervical dislocation. The first rodent removal event was directly after the mark-recapture trapping in September 2012. Thereafter, rodents that accidentally found their way into the plots were removed monthly until December 2014 using the same grid with traps. Four control plots received no treatment $(C)$. To increase rodent density, acorns were added to four plots (AA)(Sunyer et al. 2016). Acorns were provided beneath feeding stations, which were made of $60^{*} 60 \mathrm{~cm}$ plates kept $5 \mathrm{~cm}$ above the ground with 8 wooden poles to prevent acorn predation by birds and large mammals. In each plot, 16 feeding stations were placed in a $4 * 4$ grid with $15 \mathrm{~m}$ between feeding stations. Each feeding station was provided with $6.25 \mathrm{~kg}$ of acorns in November and January of 2012 and 2013 (1600 $\mathrm{kg}$ in total). Control feeding stations without acorns were placed in the control and rodent removal plots. A screen was placed around the four rodent removal plots to reduce immigration 
of rodents (Jones et al. 1998). Metal poles (8mm diameter, $1 \mathrm{~m}$ long) were placed every 5 meters and reached $40 \mathrm{~cm}$ above ground. A $3 \mathrm{~mm}$ steel cable was attached to the top of the poles. At the corners of each plot a wooden pole with a diameter of $12 \mathrm{~cm}$ was placed. A screen of two layers of $0.3 \mathrm{~mm}$ plastic was dug $10 \mathrm{~cm}$ into the ground and was attached to the cable. To overcome a possible bias in vertebrate community caused by a visual effect of the screens on birds and large mammals, screens were also placed around the control and acorn addition plots. However, the lowest $10 \mathrm{~cm}$ of these plots was left open by attaching the lower part of the screen to another $3 \mathrm{~mm}$ steel cable, enabling rodents to walk around freely (Figure 3, Chapter 1 and Figure 1).

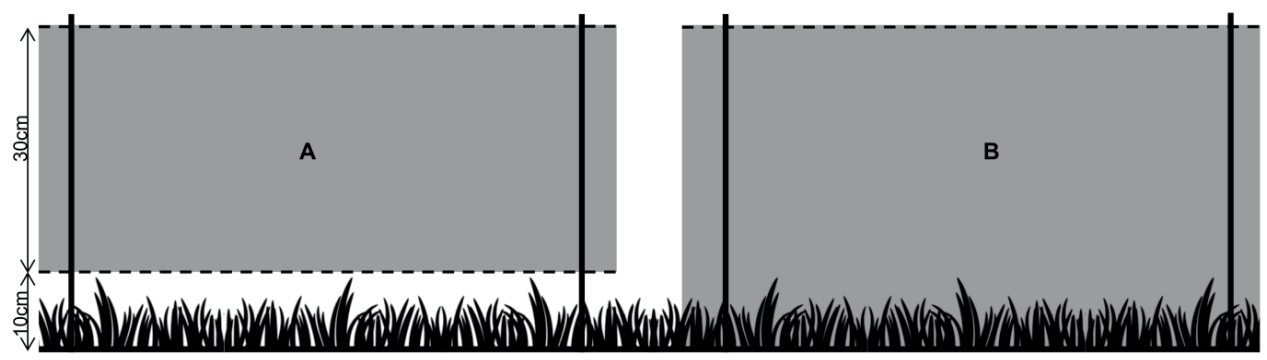

Figure 1. Schematic overview of the screens that were placed around the plots. Solid lines represent metal poles and dottet lines represent steel cables. Screen A was placed around the control plots and around the plots to which acorns were supplemented, screen B was placed around the plots from which the rodens were removed.

\section{Rodent sampling}

Rodents were sampled at 3 month intervals (March, June, September and December) from September 2012 till December 2014. At each plot, 25 Heslinga live traps were placed in a 5^5 grid. Traps were pre-baited with grain for 3 days after which they were rebaited with grain, carrot and mealworms and set at 09:00. Traps were then inspected 4 times at $12 \mathrm{~h}$ intervals. Trapped rodents were marked by cutting a piece of hair from their back (Panzacchi et al. 2010). Rodent density was calculated per species according to the Schnabel method (Schnabel 1983). When there were no recaptures in a plot in a trap month, rodent density was estimated as: (Number of trapped rodents in plot $X$ * Rodent density estimated according to Schnabel method in plots with recaptures) / (Total number of rodents trapped in plots with recaptures). During the 09:00 trappings, rodents were screened for ticks by searching the ears, snout, head, belly, legs, armpits, throat and tail. A small ear biopsy was taken with sterile scissors from each rodent and stored in $70 \%$ ethanol at $-20^{\circ} \mathrm{C}$ until further analysis. Captured shrews were released immediately. 


\section{Tick sampling}

Ticks were captured monthly in each plot by dragging a $1 * 1 \mathrm{~m}$ white cotton blanket with a chain at one side and a tube attached to a rope on the other side over the vegetation (Gassner 2010). At each plot four rows of $50 \mathrm{~m}$ were sampled and the blanket was inspected at $25 \mathrm{~m}$ intervals. All attached ticks were counted and attached nymphs were collected and stored in $70 \%$ ethanol at $-20^{\circ} \mathrm{C}$ until further analysis. Larvae and adult ticks were removed with a brush. Sampling was performed in the afternoons (12:00-18:00) when the vegetation was dry.

\section{Identification of Borrelia burgdorferi s.I. infections}

Ear biopsies and nymphs were analysed individually. A maximum of 40 nymphs per plot per month were first heated for $20 \mathrm{~min}$ at $99^{\circ} \mathrm{C}$ in $100 \mu \mathrm{l}$ ammonium hydroxide $\left(\mathrm{NH}_{4} \mathrm{OH}, 1 \mathrm{M}\right)$. Nymphs were then centrifuged and heated for $20 \mathrm{~min}$ at $99^{\circ} \mathrm{C}$ with open lids to evaporate the ammonia. The lysates were stored at $4^{\circ} \mathrm{C}$. DNA was extracted from the ear biopsies using the Qiagen DNeasy Blood \& Tissue Kit (Jahfari et al. 2014). B. burgdorferi s.I. DNA was detected using $\mathrm{qPCR}$ in the IQ Multiplex Powermix with a volume of $20 \mu \mathrm{l}$, containing iTaq DNA polymerase (Bio-Rad Laboratories, USA), $200 \mathrm{nM}$ of each primer and $5 \mu$ of template DNA (Heylen et al. 2013b). Outer surface protein A gene (OspA) (forward primer: $5^{\prime}$-AAT ATT TAT TGG GAA TAG GTC TAA-3'; reverse primer: 5'-CTTTGT CTT TTT CTT TRC TTA CA-3' and probe: 5'-Atto520-AAG CAA AAT GTT AGC AGC CTT GA-BHQ1-3') and the B. burgdorferi s.I. flagellin gene (flaB) (forward primer: 5'-CAG AIA GAG GTT CTA TAC AIA TTG AIA TAG A-3'; reverse primer: 5'-GTG CAT TTG GTT AIA TTG YGC-3' and probe: 5'-Atto425-CAA CTI ACA GAI GAA AXT AAI AGA ATT GCT GAI CA-Pho-3', where $X$ stands for an internal BHQ-1 quencher attached to thymine) were used as targets. The qPCR cycling program (using a light cycler 480 real-time PCR system, Hoffmann-La Roche, Switzerland) was performed using a two-step PCR program: Taq activation for $5 \mathrm{~min}$ at $95^{\circ} \mathrm{C}$ followed by 60 cycles of $5 \mathrm{~s}$ at $94^{\circ} \mathrm{C}$ and $35 \mathrm{~s}$ at $60^{\circ} \mathrm{C}$ involving a single point measurement at $60^{\circ} \mathrm{C}$ with corresponding filters, finishing with one cycle of $20 \mathrm{~s}$ at $37^{\circ} \mathrm{C}$.

\section{Data analysis}

The relationships between the average rodent density in the period September-December (variable $\mathrm{x}$ ) on the variables $\mathrm{y}_{1}-\mathrm{y}_{6}$, was analysed. The response variables are $\mathrm{y}_{1}$ : average density of nymphs the period May-October one year later, $y_{2}$ : average density of infected nymphs the period May-October one year later, $\mathrm{y}_{3}$ : nymphal infection prevalence one year later, calculated as $\mathrm{y}_{2} / \mathrm{y}_{1}, \mathrm{y}_{4}$ : the number of infected rodents out of the number of analysed rodents $n_{a}$ in September and December, $y_{5}$ : the total number of larvae on all caught rodents, 
in relation to the number of caught rodents $\mathrm{n}_{\mathrm{c}}$ in September and December, and $\mathrm{y}_{6}$ : the total number of nymphs on all caught rodents, in relation to the number of caught rodents $n_{c}$ in September and December. $\mathrm{Y}_{1}-\mathrm{y}_{3}$ were analysed at plot level, $\mathrm{y}_{4}-\mathrm{y}_{6}$ were analysed at rodent level.

$\log \left(y_{1}+1\right), \log \left(y_{2}+1\right)$ and $\arcsin \left(\sqrt{ }_{y_{3}}\right)$ were analysed as dependent variables using mixed linear models with fixed effects for year, covariate $\log (x+1)$ and their interaction, and random effects for plots. $Y_{4}-y_{6}$ were analysed using generalized linear mixed models (glmm's). For $\mathrm{y}_{4}$ a glmm with binomial distribution, binomial total $\mathrm{n}_{\mathrm{a}}$ and logit link function is used. For the counts $\mathrm{y}_{5}$ and $\mathrm{y}_{6}$ a glmm with Poisson distribution, log link functions and offset $\log \left(\mathrm{n}_{\mathrm{c}}\right)$ is used. Fixed effects for year, covariate $\log (x+1)$ and their interaction are taken, and random effects for plots. An extra random effect for individual observations was used in the glmm for $\mathrm{y}_{5}$ to correct for overdispersion.

$\mathrm{Y}_{1}-\mathrm{y}_{3}$ were analysed with ordinary linear mixed models for transformed responses, and $\mathrm{y}_{4}-\mathrm{y}_{6}$ with glmm's because the of the large variability in rodent counts over plots and its effect on the variability of responses $\mathrm{y}_{4}-\mathrm{y}_{6}$, which would remain unhandled otherwise. Results from the mixed models are shown in plots, by fitting simplified models after removal of non-significant terms, and back transforming predicted values to the original count or fraction scales. All analyses were performed in $\mathrm{R}$ version 3.3.0, using specifically the package Ime4 for (generalized) linear mixed models.

\section{Results}

\section{Climate}

Mean monthly temperature was lowest during the winter of 2012/2013, highest during the winter of 2013/2014 and medium during the winter of 2014/2015 (Appendix 1). Mean monthly saturation deficit was comparable between years but varied throughout the seasons.

\section{Effect of treatment on rodent density}

Rodent density varied throughout the study period and was affected by treatment in December 2012, March 2013, September 2013, December 2013, March 2014 and June 2014 ( $P<0.05$, Figure 2). At these moments, the removal of rodents led to a lower rodent density and the supplementation with acorns led to a higher rodent density than in the control plots. Rodent density was not affect by treatment in the second half of 2014 . 


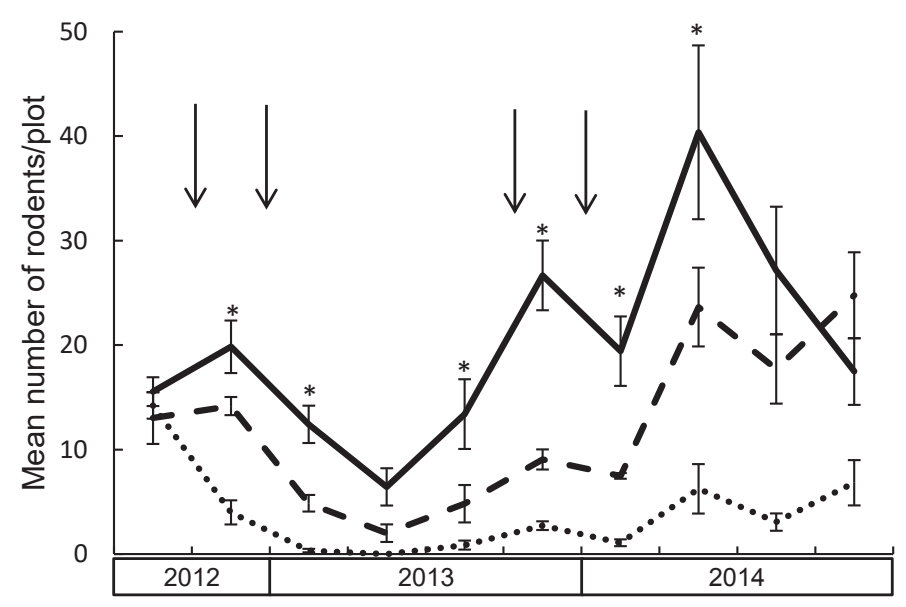

Figure 2. Mean number of rodents per plot of plots that were supplied with acorns (solid line), control plots (dashed line) and plots from which the rodents were removed (dotted line). Arrows indicate events of acorn supplementation. Error bars represent SE of 4 plots. Asterisks indicate an effect of treatment on rodent density $(P<0.05)$.

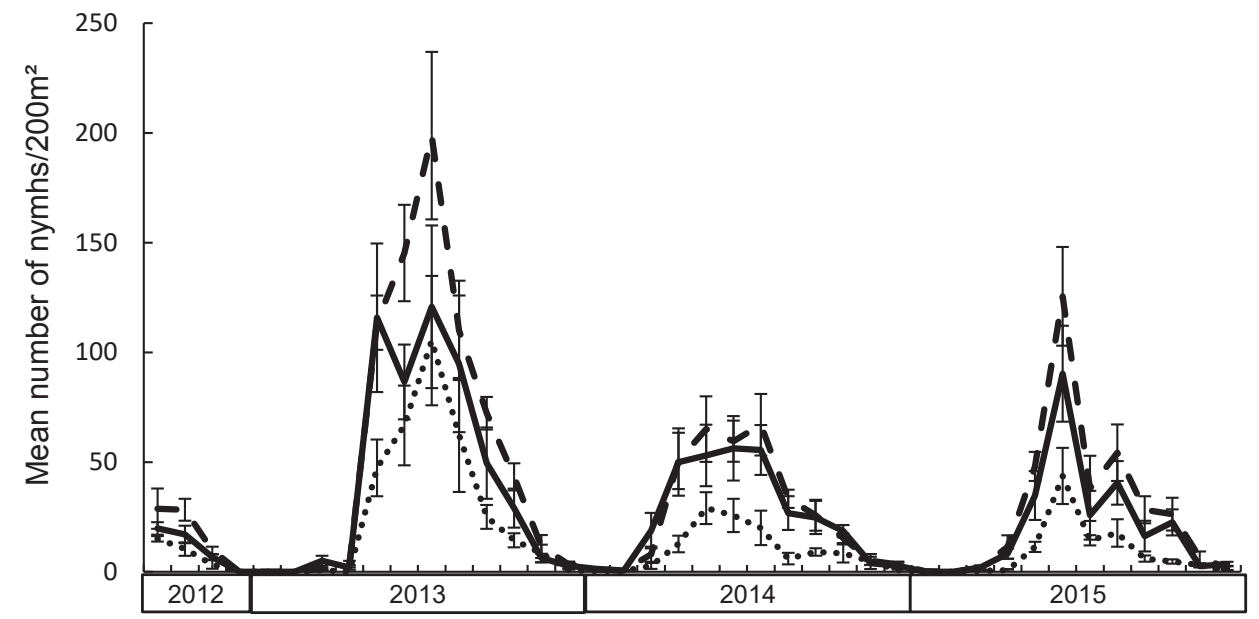

Figure 3. Mean number of questing nymphs per $200 \mathrm{~m}^{2}$ of plots that were supplied with acorns (solid line), control plots (dashed line) and plots from which the rodents were removed (dotted line). Error bars represent SE of 4 plots.

\section{Effect of rodent density on the DIN}

DON varied within and between years and was highest from May till October (Figure 3). The figure shows that DON was reduced in the rodent removal plots, whereas DON in the acorn supplementation plots was not higher than in the control plots. The statistical model, where the effect of rodent density irrespective of treatment was analysed, showed that rodent density had a positive effect on $\mathrm{DON}$ one year later $(\mathrm{P}=0.0004$, Figure $4 \mathrm{~A})$. $\mathrm{DON}$ also varied between years 
$(P<0.0001)$ and was higher in year 1 compared to year $2(P<0.0001)$ and year $3(P<0.0001)$. $\mathrm{DON}$ in year 2 and year 3 were not statistically different from each other $(P=0.2820)$. Rodent density did not affect NIP one year later $(P=0.3432$, Figure $4 B)$ and year had no effect on NIP $(P=0.8094)$. Rodent density had a positive effect on DIN one year later $(P=0.0018$, Figure $4 C)$. DIN also varied between years $(P<0.0001)$ and was higher in year 1 compared to year 2 $(P=0.0007)$ and year $3(P=0.0001)$. DIN in year 2 and year 3 were not different from each other $(\mathrm{P}=0.7579)$. Rodent density had a positive effect on the $B$. burgdorferi s.l. infection prevalence of rodents in the same year (Figure $5 \mathrm{~A}, \mathrm{P}=0.0020$ ). Infection prevalence of rodents did not differ between years $(P=0.3014)$. Rodent density had an effect on mean larval tick burden of rodents in the same year and the effect differed between years $(P=0.0132)$. The effect was positive in 2012, whereas it was negative in 2013 and 2014 (Figure 5B). Rodent density had a positive effect on the mean nymphal tick burden of rodents in the same year $(P=0.0127$, Figure $5 C)$. Year had no effect on nymphal tick burdens on rodents $(P=0.4923)$. 

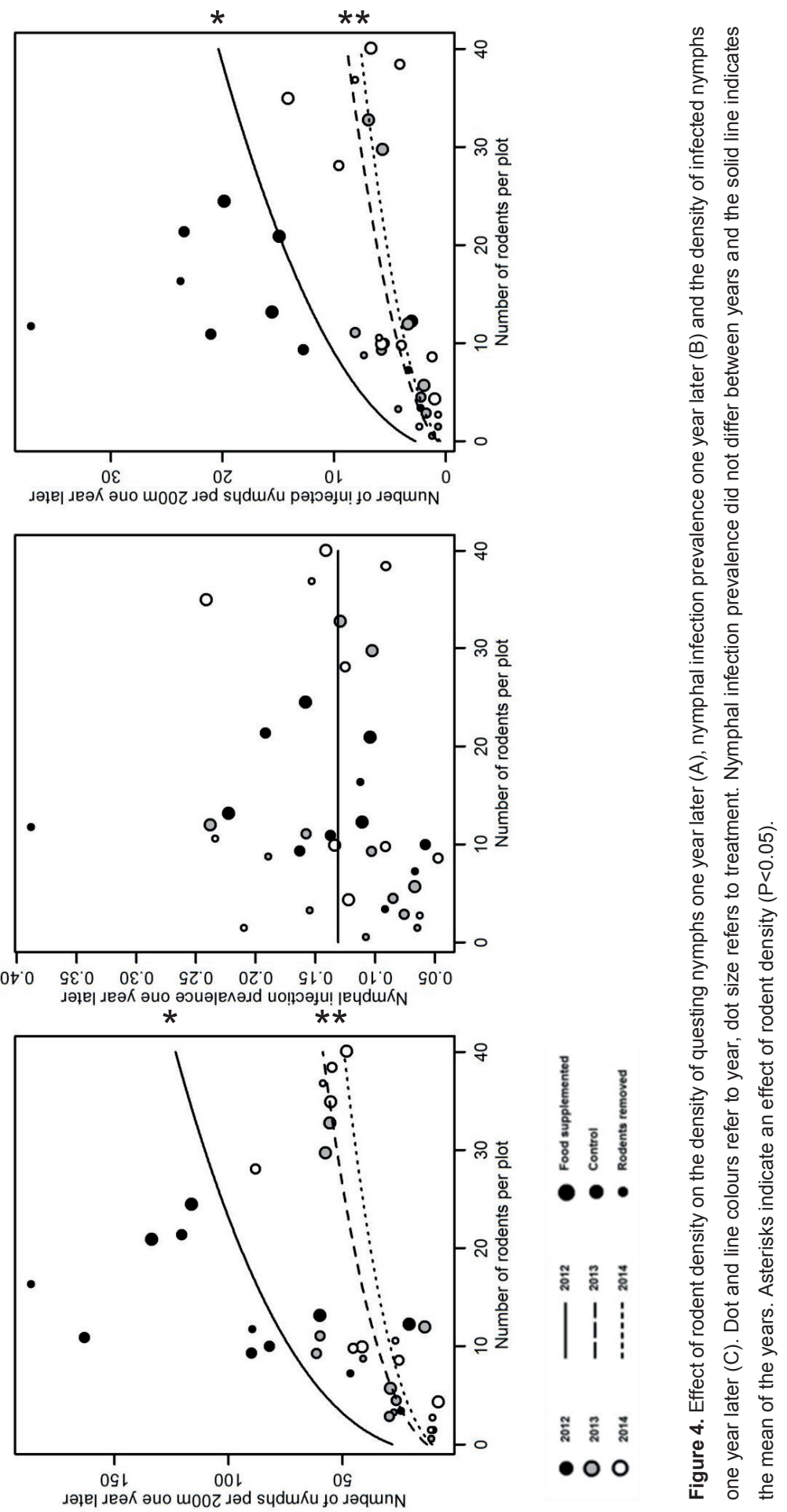

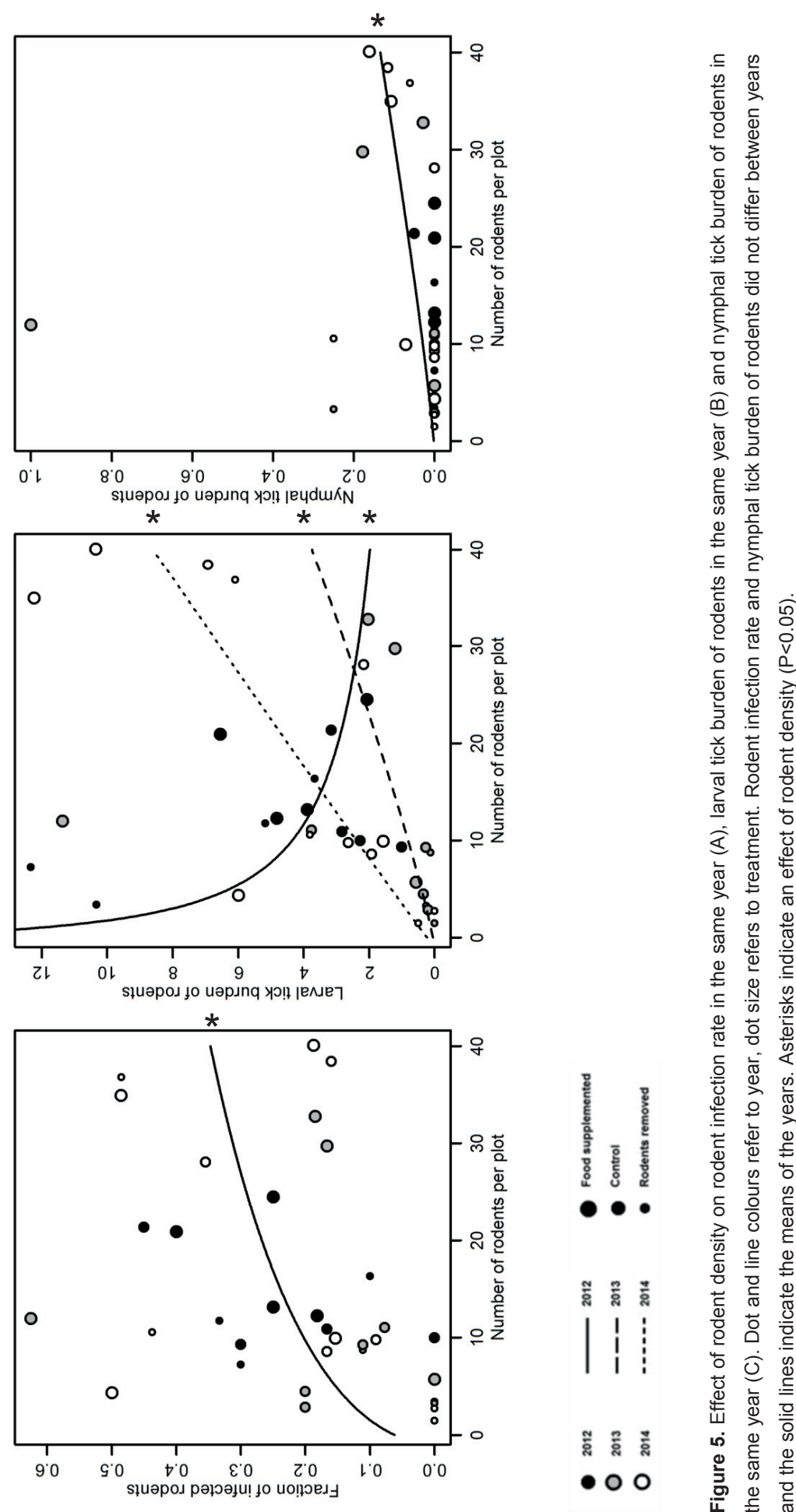

วิ

$1 \mathrm{I}$ 


\section{Discussion}

The results of the study demonstrate that rodent density has a positive effect on DIN one year later. This effect was caused by an increase in DON, whereas NIP was not affected. A positive effect of rodent density on DIN the following year was also found in an observational study by Ostfeld et al. (2001). These authors, however suggested that the increased DIN was caused by an increased deer population because addition of acorns also attracts deer (Ostfeld et al. 1996, Jones et al. 1998). In our experimental study, however, acorns were supplied under feeding stations, protecting them from predation by deer and other large grazers. Acorn availability in autumn, therefore, affects Lyme borreliosis incidence two years later (Bogdziewicz and Szymkowiak 2016), as a result of an increased rodent density one year later and DIN two years later (Stafford III et al. 1998). Previously, most studies on the relationship between rodent density and DIN were done in the USA with Peromyscus leucopus as rodent species and $I$. scapularis as tick species, whereas in our study site in western Europe more rodent species are used and $I$. ricinus was the dominant tick species. This relationship, therefore appears, to be a common paradigm in the ecology of Lyme borreliosis, and enhances the role of rodents in Lyme borreliosis risk.

In western Europe, rodents and larvae collected from rodents are mainly infected with $B$. afzelii (Humair et al. 1999, Hanincová et al. 2003a, Kybicova et al. 2008, Schmidt et al. 2014b). Rodents can, however, also be infected with $B$. bavariensis $B$. burgdorferi sensu stricto and Borrelia spielmanii (Kurtenbach et al. 1994, Humair et al. 1995, Richter et al. 2011, Coipan et al. 2016). In The Netherlands, B. afzelii is the most prevalent B. burgdorferi s.l. genospecies found in I. ricinus nymphs (Gassner et al. 2011, Coipan et al. 2016). Because not all genospecies are associated with rodents (Kurtenbach et al. 2002a, Hanincová et al. 2003b), DIN is likely to be affected by other vertebrates as well. The composition of genospecies is, therefore, affected by fauna composition and tick feeding behaviour. Our molecular analysis did not distinguish between $B$. burgdorferi s.I. genospecies, so therefore the role of the various $B$. burgdorferi s.I. genospecies in contributing to DIN requires further study.

We investigated the effect of the total rodent population on DIN and did not incorporate any characteristics of the rodents (e.g. species, sex). Host characteristics may, however, have an influence on the suitability of a rodent as host for I. ricinus and $B$. afzelii and, therefore, on its contribution to DIN (Van Duijvendijk et al. 2015). Wood mice and bank voles were the only rodent species trapped in our study and are the most common rodent species in the area (Gassner et al. 2013). The effect of rodent density on DON can differ between seasons and rodent species. In spring, wood mouse density had a positive effect, whereas the density of bank voles had a negative effect on DON one year later (Perez et al. 2016). In autumn, 
however, bank vole density had a positive effect on the DON one year later (Perez et al. 2016). The fluctuation in rodent density throughout the season was comparable with the fluctuations in other woodland areas (Montgomery 1989). Fluctuations in population density may, however, vary between wood mice and bank voles (Mallorie and Flowerdew 1994). We found that rodent density responds to acorn availability in the preceding winter (Figure 2). Wood mice and bank voles are known to hoard seeds (Mappes 1998, Perea et al. 2011) and their densities in the plots supplemented with acorns probably increased due to an increased reproduction and winter survival (Pucek et al. 1993, Díaz and Alonso 2003). It was shown that wood mice respond strongly to acorn availability, whereas bank vole population fluctuations respond more to the availability of bilberries (Vaccinium myrtillus) and spruce seeds (Picea abies) (Selås et al. 2002), so it is possible that in our study only wood mice responded to the acorn supplementation.

We found a later peak in rodent density in 2013 compared to 2014 , which was probably caused by a lower summer population during the summer of 2013 (Montgomery 1989). This caused less overlap with the peaks in density of questing I. ricinus larvae and nymphs (Randolph et al. 2002), but had no effect on rodent infection rate. Rodent density decreased in the summer of 2014 in the plots where acorns had been given as supplementary food. This was likely to be caused by a shortage of food (Krebs 2013) due to the relatively high rodent density caused by the supplemented acorns in the preceding winter. After the supply of acorns had been exhausted, rodent density was too high to survive on the naturally available food in the environment. This resulted in either emigration or increased rodent mortality due to starvation (Stradiotto et al. 2009, Sunyer et al. 2016).

DON and DIN were higher in 2013 compared to 2014 and 2015, which appears not to be the result of a difference in winter or summer temperature. It may have been caused by an effect of the screens surrounding the plots on the abundance of hosts for adults ticks in the plots in previous year. The screens were placed around all plots, so these are expected not to differ between treatments and plots.

There was a positive effect of rodent density on rodent infection rate. This may have been caused by an increased survival of rodents in the plots supplemented with acorns because infected rodents stay infective throughout their lives (Gern et al. 1994, Humair et al. 1999). Older rodents are, therefore, more likely to be infected due to a higher likelihood of being exposed to infected ticks. The addition of acorns may also have increased rodent activity (Sunyer et al. 2016), which may in turn have increased their exposure to infected ticks. A higher rodent infection rate, caused by a higher rodent density, could result in an higher NIP one year later. Rodent density had, however no effect on NIP. We found a dilution effect of rodent density on larval tick burden in 2012, whereas a magnifying effect was found in 2013 and 2014. These opposing effects may have been caused by a delay in the effect of treatment. Mean 
rodent density in September/December was used as a proxy for rodent density of that year (because the first sampling was in September 2012). Treatment, however, only affected rodent density from December 2012 onwards. The dilution effect in 2012 could, therefore, have been the result of the natural, unaffected rodent population in September 2012. The highest rodent densities in 2012 were indeed lower compared to the highest rodent densities in 2013 and 2014. The magnifying effect in 2013 and 2014 could be the result of an increased rodent activity in plots with supplemented acorns (see above). The magnifying effect of rodent density on larval and nymphal tick burden probably caused the increased rodent infection rate with an increased rodent density. A higher tick burden would result in a higher exposure to infected larvae and nymphs and, therefore, a higher rodent infection rate (Radolf et al. 2012, Van Duijvendijk et al. 2016). The effect of rodent density on rodent infection rate was, however, not significantly affected by year and NIP was also not affected by rodent density. An inconsistent effect of artificial manipulated host density (Tamias striatus) on tick burden (I. scapularis) was also found by Schmidt et al. (1999) and a negative effect of rodent density on tick burdens on rodents was reported by Rosa et al. (2007). Schmidt et al. (1999) suggested that either tick behaviour or host responses affect the effect of rodent density on their tick burden. Despite the dilution effect of rodent density on their tick burden, the total number of feeding larvae on the rodent population, which affects DIN one year later, is likely to increase with an increase in rodent density (Rosa et al. 2007).

We conclude that rodent density has a positive effect on DIN one year later and that this effect is caused by an increase in DON, whereas NIP is not affected. The results underline the importance of rodents as blood hosts for $I$. ricinus larvae. Further research is required to investigate which rodents contribute most to DIN. Our findings provide important information for the prediction of Lyme borreliosis risk.

\section{Acknowledgements}

We thank everybody who helped with the rodent trappings and Natuurmonumenten and Gemeente Ede for providing permission to conduct the experiment in their forests. 



\title{
Chapter 4
}

\section{Interactions between Ixodes ricinus,} rodents and Borrelia burgdorferi

\author{
sensu lato affecting Lyme
}

borreliosis risk in a natural

ecosystem

Gilian van Duijvendijk, Inge Krijger, Marloes van Schaijk, Manoj Fonville, Gerrit Gort, Hein Sprong, Willem Takken 


\section{Abstract}

Lyme borreliosis is caused by Borrelia burgdorferi sensu lato (s.I.), which is transmitted by Ixodes ricinus. Borrelia miyamotoi causes Borrelia miyamotoi disease in humans and is transmitted by the same tick species. Ixodes ricinus larvae mainly feed on rodents and can acquire the spirochaetes from infected rodent during feeding. The contribution of rodents to the density of infected nymphs is heterogeneous and is affected by their 1) larval tick burden, 2 ) infection rate, 3) infectivity, and 4) the moulting success of engorged larvae. The aim of this study was to identify the factors that affect the contribution of rodents to the density of infected nymphs. Rodents were live trapped and analysed for ticks from May to November in 2013 and 2014 in two research plots. Male rodents were temporarily housed in the laboratory and naturally detached ticks were collected. Borrelia burgdorferi s.I. and B. miyamotoi infections were determined from the trapped rodents and collected ticks.

Rodent density was about one order of magnitude higher in 2014 compared to 2013, whereas larval and nymphal tick burden and B. burgdorferi s.I. rodent infection rate were lower in 2014 compared to 2013. Consequently, the contribution of the 2013 rodent population to the density of $B$. burgdorferi s.l.-infected nymphs was only slightly lower than the contribution of the 2014 rodent population. The contribution to the density of $B$. miyamotoi-infected nymphs did not differ between years. We found higher larval and nymphal tick burdens on wood mice (Apodemus sylvaticus) compared to bank voles (Myodes glareolus) and on males compared to females. Larval tick burden and $B$. burgdorferi s.l. rodent infection rate peaked in summer. Rodent infectivity with $B$. burgdorferi s.l. was not affected by any of the measured variables.

We conclude that the risks for Lyme borreliosis and Borrelia miyamotoi disease, expressed as the density of infected nymphs are, therefore, not directly affected by a temporal variation in rodent density. 


\section{Introduction}

Lyme borreliosis is caused by Borrelia burgdorferi sensu lato (s.I.), which is, in Europe, transmitted by the sheep tick (Ixodes ricinus). Borrelia miyamotoi causes Borrelia miyamotoi disease in humans and is transmitted by the same tick species. After hatching from the egg, I. ricinus larvae search for a vertebrate host. Rodents are commonly used as blood host by larvae and $B$. burgdorferi s.l. spirochaetes can be transmitted from infected rodent to feeding larvae (Hofmeester et al. 2016). Infected engorged larvae moult into infected nymphs, which can transmit the spirochaetes to rodents, thus closing the $B$. burgdorferi infection cycle among rodents. In contradiction to $B$. burgdorferi s.I., Borrelia miyamotoi is not only transmitted from rodent to larva (Burri et al. 2014), but also from female tick to larva (Scoles et al. 2001). The lifecycle of $B$. miyamotoi may, therefore, be less dependent on rodents (Barbour et al. 2009). Ixodes ricinus can, at a low level, be coinfected with both pathogens (Kiewra et al. 2014, Kjelland et al. 2015). Ticks are highly aggregated among rodents and a minority of rodents feeds the majority of the ticks (Perkins et al. 2003), following the Pareto principle or the 20/80 rule (Woolhouse et al. 1997). A high burden of infected ticks increases the likelihood of rodents being infected with $B$. burgdorferi s.l., and a high larval tick burden on infected rodents increases the infection prevalence in questing nymphs. It is expected that that the contribution of rodents to the density of infected nymphs is, therefore, also heterogeneous (Harrison and Bennett 2012). The contribution of an individual rodent to the density of infected nymphs is affected by 1) the number of larvae that feeds on the rodent (larval tick burden), 2) the chance that the rodent is infected (rodent infection rate), 3) the chance that the spirochaetes are transmitted to feeding larvae (rodent infectivity), and 4) the chance that the engorged larvae moult into nymphs (moulting success). These four factors, in turn, may be affected by different biotic and abiotic factors, which may differ temporally and geographically (Li et al. 2012, Perez et al. 2012).

Rodent species differ in their suitability as host for ticks and host for B. burgdorferi s.l. (Nilsson and Lundqvist 1978, Matuschka et al. 1992a, Kurtenbach et al. 1994). Tick burden also varies within rodent species, which may be due to differences in rodent characteristics (age, sex, immune status), microclimate or vegetation composition (Randolph and Storey 1999, Gassner et al. 2011). Climate and season are known to influence the behaviour of both ticks and rodents, and can also affect tick burdens on rodents (Hubalek et al. 1994, Estrada-Peña et al. 2013). The spatial aggregation of questing larvae in the environment may also contribute to the heterogeneity in larval tick burdens. Tick burden on white footed mice (Peromyscus leucopus) was, however, consistent over time per individual mouse and was only slightly affected by this spatial aggregation (Devevey and Brisson 2012), suggesting a large effect of the rodent characteristics on rodent tick burdens. The interactions between ticks, rodents and 
B. burgdorferi s.I. are complex (Van Duijvendijk et al. 2015), whereas even less is known about the ecology of $B$. miyamotoi. Most studies investigated only one or a few variables that potentially explain tick burdens on rodents. These variables may, however, also interact with each other. The relative importance of each variable can, therefore, not be compared, but is important to understand the ecology of the parasite communities. The aim of this study was to identify the factors that affect the contribution of rodents to the density of infected nymphs (DIN). The study was conducted over several successive months in two years in two research plots to include the spatiotemporal variation of the ecosystem on the factors being examined.

\section{Materials and methods}

\section{Study sites}

The study was conducted in a forest near Wageningen, The Netherlands. Two plots were selected with an inter-plot distance of $350 \mathrm{~m}$. Undergrowth of plot A $\left(51^{\circ} 59^{\prime} 35.43^{\prime \prime} \mathrm{N}\right.$, $5^{\circ} 43^{\prime} 42.06 " \mathrm{E}$ ) was dominated by wavy hair-grass (Deschampsia flexuosa), broad buckler-fern (Dryopteris dilatata) and blackberry (Rubus fruticosus) and of plot B $\left(51^{\circ} 59^{\prime} 37.22 " \mathrm{~N}\right.$, $5^{\circ} 43^{\prime 22.08 " E) ~ b y ~ b r o a d ~ b u c k l e r-f e r n ~(D r y o p t e r i s ~ d i l a t a t a) ~ a n d ~ b l a c k b e r r y ~(R u b u s ~ f r u t i c o s u s) . ~}$ Dominant tree species in both plots were scots pine (Pinus sylvestris) and silver birch (Betula pendula). The forest was part of a protected nature conservation area. Common mammal species present were bank vole (Myodes glareolus), wood mouse (Apodemus sylvaticus), common shrew (Sorex araneus), hare (Lepus europaeus), fox (Vulpes vulpes), badger (Meles meles) and roe deer (Capreolus capreolus). Bird populations consisted of a blend of species common to mixed forests of north-western Europe.

\section{Rodent trapping}

Rodents were trapped with Heslinga live traps provided with hay, grain, carrot and a few mealworms. Traps were placed at three-week intervals at 15:30 and inspected the next day at 08.30 from May till November in 2013 and 2014 (10 trapping weeks each year) at two plots. In 2013 plot B was started in July (7 trapping weeks). Traps were placed at $5 \mathrm{~m}$ inter-trap distance in grids of $12 * 12$ traps in 2013 and 6*12 traps in 2014. Trapped rodents were identified to species level, sexed, weighed and screened for ticks by searching the ears, snout, head, belly, legs, armpits, throat and tail. A small ear biopsy was collected from each captured male rodent with sterile scissors and tweezers during the first capture in 2013 and from males and females during each capture in 2014 . Ear biopsies were stored in $70 \%$ alcohol at $-20^{\circ} \mathrm{C}$ until further analysis. Each rodent was individually marked with a unique haircut. Host reference number 
and trap number were recorded for each rodent. A maximum of 15 male rodents per species was transported to the laboratory for the collection of naturally detached ticks. Captured shrews were released immediately. All experiments were approved by the ethical committee of Wageningen University (permit number 2013017 and 2014064).

\section{Rodent housing and tick collection}

Trapped rodent males that were transported to the laboratory were housed individually in Makrolon type II cages over pans with water with petroleum jelly on the edge. Cages were provided with standard rodent bedding and a tissue and toilet roll for shelter. Food and water were provided ad libitum. After 3 and 6 days, engorged ticks were collected from the water, dried on filter paper for $2 \mathrm{hr}$ and housed in ventilated $0.2 \mathrm{ml}$ Eppendorf tubes at $20^{\circ} \mathrm{C}, 90-95 \%$ $\mathrm{RH}$ and a photoperiod of $14 \mathrm{~L}: 10 \mathrm{D}$. Engorged ticks were checked weekly and were stored at $20^{\circ} \mathrm{C}$ after they moulted to the next developmental stage until further analysis. In 2013, all emerged ticks were identified to species level. After 6 days, each rodent was released at the place of capture.

\section{Identification of B. burgdorferi s.I. and B. miyamotoi infections.}

Ear biopsies and emerged nymphs were analysed individually as described in chapter 3 : Nymphs were heated for $20 \mathrm{~min}$ at $99^{\circ} \mathrm{C}$ in $100 \mu \mathrm{l}$ ammonium hydroxide $(\mathrm{NH} 4 \mathrm{OH}, 1 \mathrm{M})$, after which they were centrifuged and heated for $20 \mathrm{~min}$ at $99^{\circ} \mathrm{C}$ with open lids to evaporate the ammonia. The lysates were stored at $4^{\circ} \mathrm{C}$. DNA was extracted from the ear biopsies using the Qiagen DNeasy Blood \& Tissue Kit (Jahfari et al. 2014). Borrelia burgdorferi s.I. DNA was detected using qPCR in the IQ Multiplex Powermix with a volume of $20 \mu$, containing iTaq DNA polymerase (Bio-Rad Laboratories, USA), $200 \mathrm{nM}$ of each primer and $3 \mu \mathrm{l}$ of template DNA (Heylen et al. 2013b). Outer surface protein A gene (OspA) (forward primer: 5'-AAT ATT TAT TGG GAA TAG GTC TAA-3'; reverse primer: 5'-CTTTGT CTT TTT CTT TRC TTA CA-3' and probe: 5'-Atto520-AAG CAA AAT GTT AGC AGC CTT GA-BHQ1-3') and the B. burgdorferi s.l. flagellin gene (flaB) (forward primer: 5'-CAG AIA GAG GTT CTA TAC AIA TTG AIA TAG A-3'; reverse primer: 5'-GTG CAT TTG GTT AIA TTG YGC-3' and probe: 5'-Atto425-CAA CTI ACA GAI GAA AXT AAI AGA ATT GCT GAI CA-Pho-3', where $X$ stands for an internal BHQ1 quencher attached to thymine) were used as targets. The qPCR cycling program (using a light cycler 480 real-time PCR system, Hoffmann-La Roche, Switzerland) was performed using a two-step PCR program: Taq activation for $5 \mathrm{~min}$ at $95^{\circ} \mathrm{C}$ followed by 60 cycles of $5 \mathrm{~s}$ at $94^{\circ} \mathrm{C}$ and $35 \mathrm{~s}$ at $60^{\circ} \mathrm{C}$ involving a single point measurement at $60^{\circ} \mathrm{C}$ with corresponding filters, 
finishing with one cycle of $20 \mathrm{~s}$ at $37^{\circ} \mathrm{C}$. In the same qPCR, B. miyamotoi was detected with primers and probe based on the flagellin gene (flaB) for detection of the bacteria.

\section{Data analysis}

Data were analysed with a generalized linear mixed model with a binomial distribution. Rodent identification number and trap number were used as random factors in each model. First, the effects of the main factors (year, rodent species, rodent sex, rodent weight, week, week ${ }^{2}$, rodent infection and plot) and all possible two-factor interactions between the main factors were analysed in four different full models (Appendix 2).

The number of larvae counted in the field was used as dependent variable in the model to analyse larval tick burden on rodents. This model included all main factors and interactions. Data from ear biopsies were used as dependent variable in the model to analyse rodent infection rate. Rodent infection and the year * rodent sex interaction were excluded from this full model. The number of $B$. burgdorferi s.l.-infected ticks out of the number of analysed ticks that were collected from the male rodents which were found $B$. burgdorferi s.l.-positive by PCR was used as dependent variable in the model to analyse rodent infectivity. Rodent infection was excluded from this full model. The number of moulted ticks out of the number of analysed ticks that were collected from the male rodents was used as dependent variable in the model to analyse larval moulting success.

Second, each model was reduced by backwards elimination of the fixed factors, starting with the highest $P$-values until all factors were either significant, or were included in an interaction factor that was significant. Effects were considered significant at $P<0.05$. All analyses were performed in SAS statistical software (SAS Institute Inc. (SAS 2004)), version 9.3.

\section{Results}

\section{Rodent density}

All trapped rodents were bank voles or wood mice (Table 1). In 2013, 78 bank voles and 51 wood mice were trapped during 2448 ( 1 plot * 144 traps * 7 nights +1 plot * 144 traps * 10 nights) trap nights. In 2014, 443 bank voles and 287 wood mice were trapped during 1440 (2 plots * 72 traps * 10 nights) trap nights. Rodent density, analysed as number of rodents trapped per trap-night, was ten-fold lower in 2013 (0.053, 129/2448) compared to 2014 (0.507, 730/1440). 


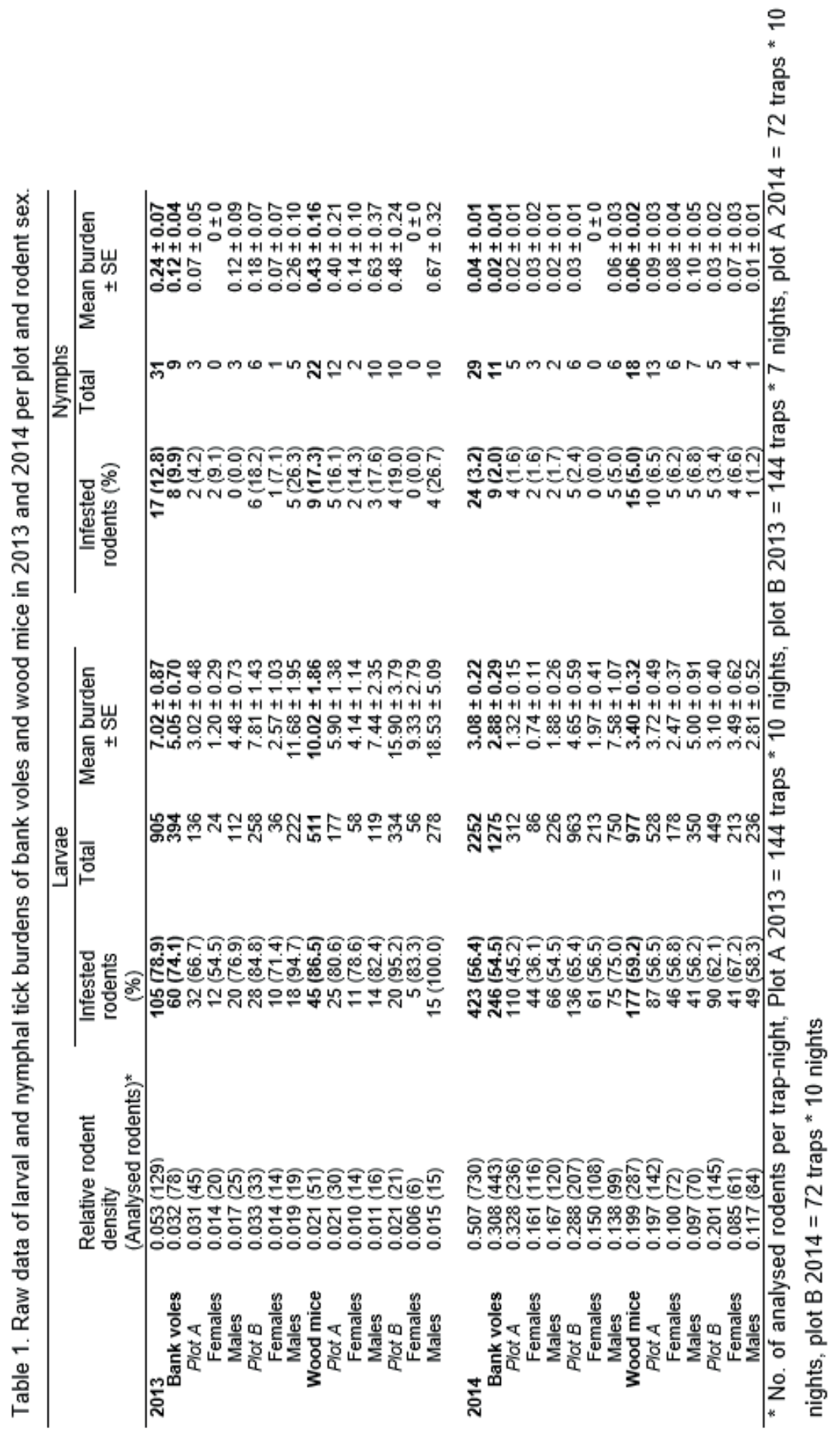




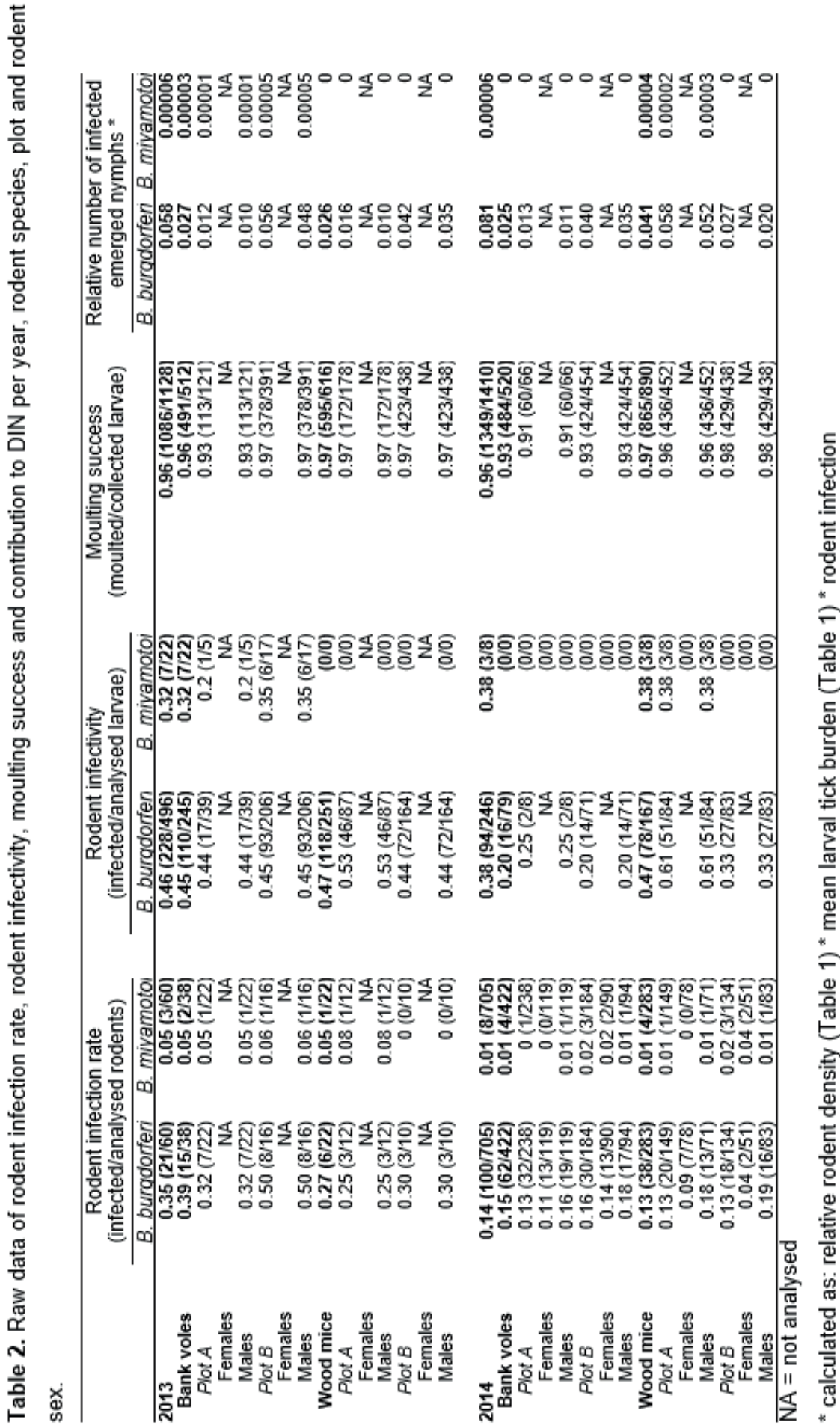




\section{Tick burden}

In total, 3,157 larvae were counted in the field on the trapped rodents (Table 1). Mean larval tick burden was $7.02 \pm 0.87$ in 2013 and $3.08 \pm 0.22$ in 2014. Larvae were highly aggregated among rodents; in $2013,20 \%$ of the rodents fed $62.3 \%$ of the larvae and in $2014,19 \%$ of the rodents fed $73.4 \%$ of the larvae (Figure 1). In 2013, 1,128 larvae were collected in the laboratory from male rodents, from which 1,086 were identified to species level after they moulted into nymphs. One of these was I. trianguliceps, all others were I. ricinus. In 2013, 33 nymphs were collected in the laboratory from male rodents, from which 17 were identified to species level after they moulted into adults. Two of these were I. trianguliceps, all others were I. ricinus. No adult ticks were collected from the water basins, even though four adult ticks were observed feeding on the rodents during trapping.

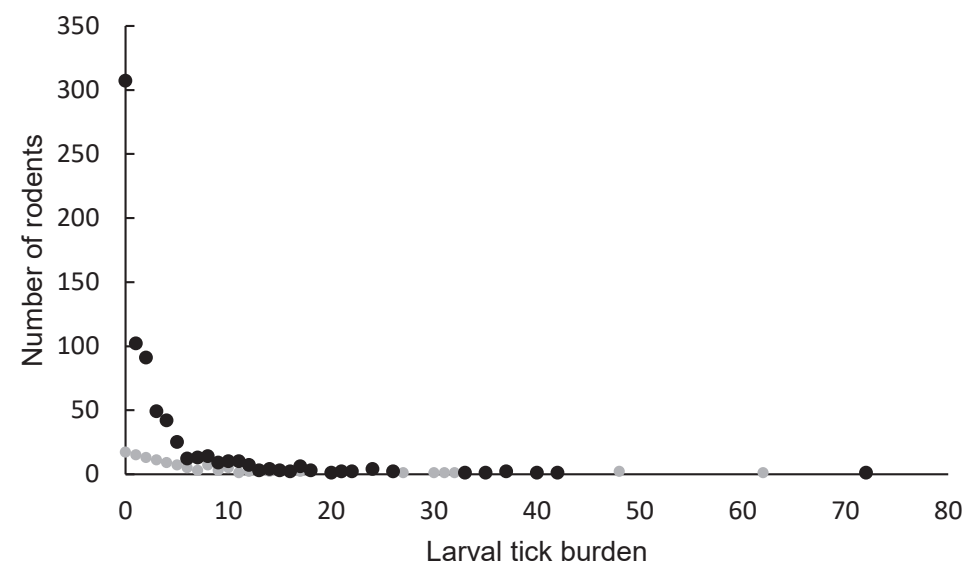

Figure 1. Number of rodents against larval tick burden (field counts) in 2013 (black) and 2014 (grey). 
Larval tick burden differed between years and was higher in 2013 (8.9, Cl: 6.9-11.4) compared to 2014 (2.9, Cl: 2.5-3.4, P<0.0001, Figure 2A). Larval tick burden differed between rodent species and was lower on bank voles (3.3, Cl: 2.7-4.0) compared to wood mice (7.9, Cl: 6.39.7, $\mathrm{P}<0.0001$, Figure 2B).
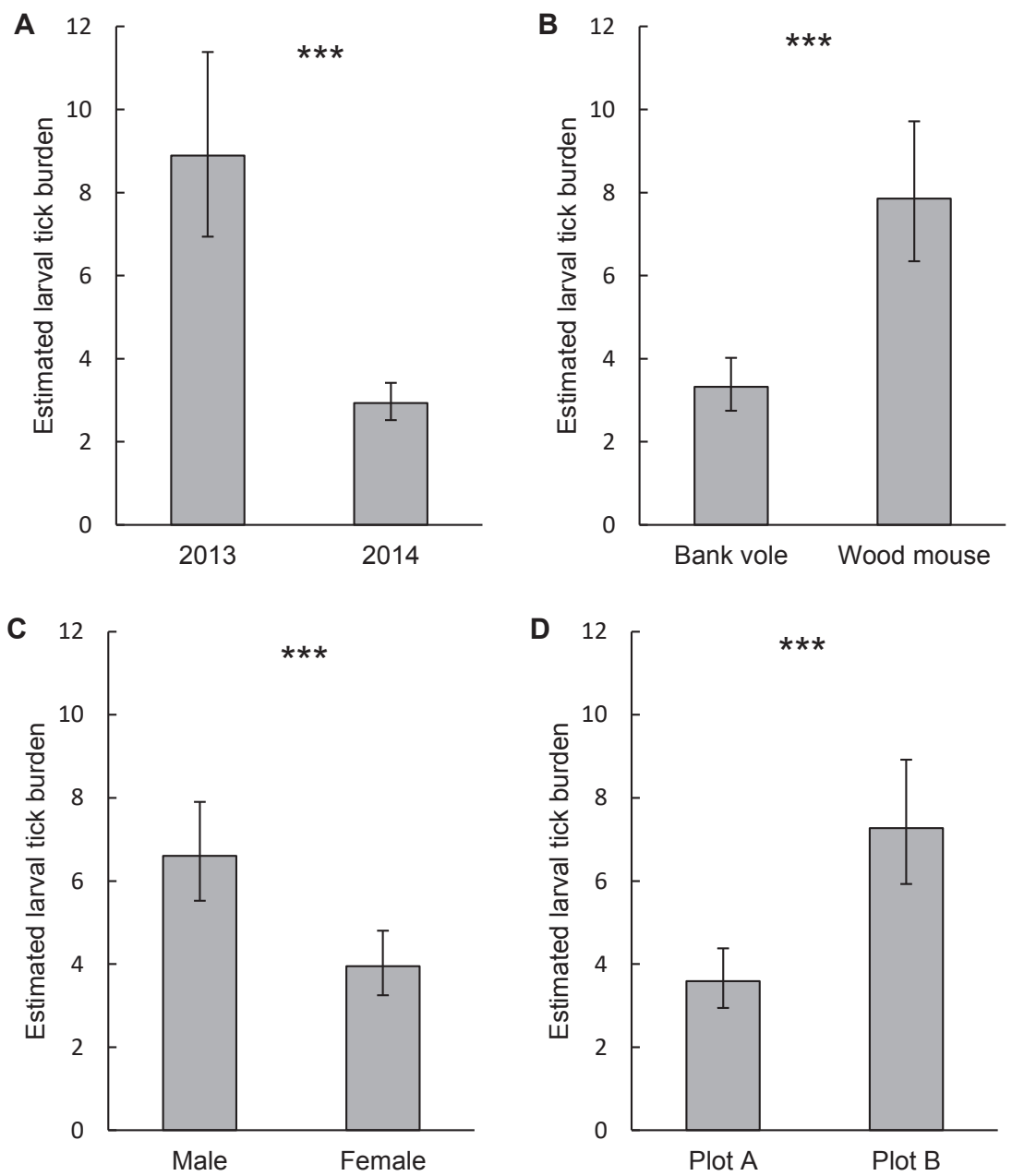

Figure 2. Estimated larval tick burden on rodents in week 32 per $(A)$ year, $(B)$ rodent species, $(C)$ rodent sex, and (D) plot. Error bars represent $95 \% \mathrm{Cl}$. Significant indications are illustrated as ${ }^{* *}=P<0.001$. 
The difference in larval tick burden between rodent species varied between plots $(P=0.0021)$; on average, bank voles had lower larval tick burdens compared to wood mice, but the effect of rodent species on larval tick burden was larger in plot $A(P<0.0001)$ compared to plot $B$ $(P<0.0011$, Figure 3). The difference in larval tick burden between rodent species varied over the trapping period $(P=0.0039$, Figure 4$)$; the difference was larger in summer than in spring and autumn.

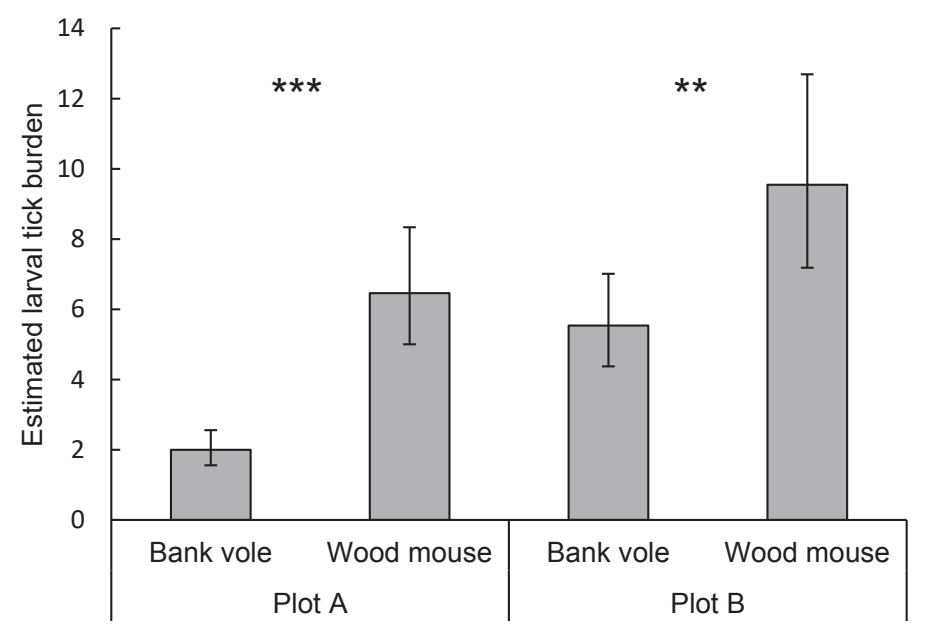

Figure 3. Estimated larval tick burden on rodents in week 32 per rodent species per plot. Error bars represent $95 \%$ Cl. Significant indications are illustrated as ${ }^{* *}=P<0.01,{ }^{* * *}=P<0.001$.

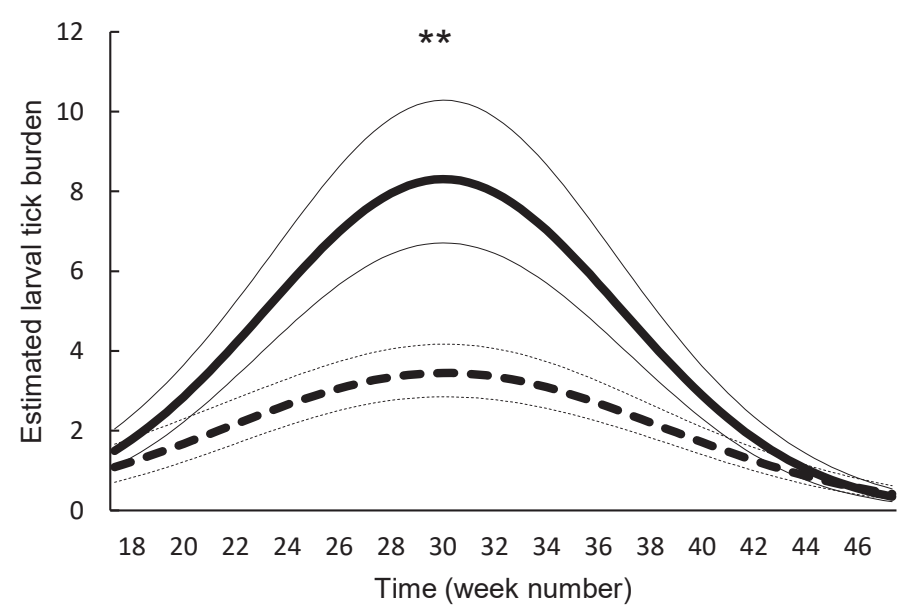

Figure 4. Estimated larval tick burden on wood mice (solid line) and bank voles (dashed line) over time. Thin lines represent upper and lower bounds of the $95 \% \mathrm{Cl}$. Significant indications are illustrated as ** $=\mathrm{P}<0.01$. 
Larval tick burden differed between rodent sexes and was higher on males (6.6, Cl: 5.5-7.9) compared to females (3.9, Cl: 3.2-4.8, $\mathrm{P}<0.0001$, Figure $2 \mathrm{C}$ ). The difference in larval tick burden between rodent sexes varied between rodent species $(P<0.0001)$; in bank voles, males had higher larval tick burdens compared to females $(P<0.0001)$, whereas sex did not affect larval tick burden on wood mice $(P=0.6366$, Figure 5$)$.

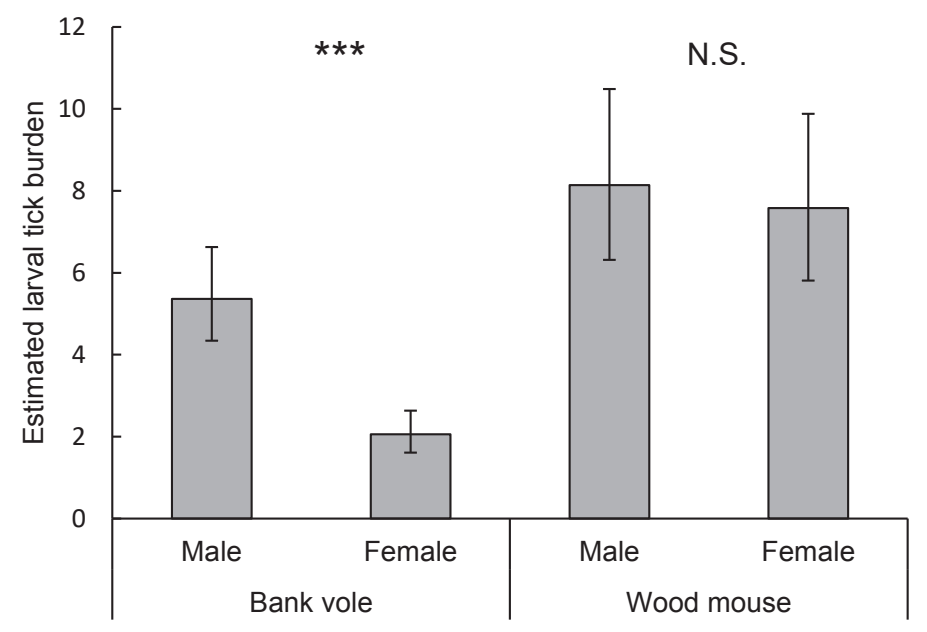

Figure 5. Estimated larval tick burden on rodents in week 32 per rodent sex per rodent species. Error bars represent $95 \%$ Cl. Significant indications are illustrated as ${ }^{* *}=P<0.001$, N.S. $=$ Not significant.

Larval tick burden differed between plots, and was lower in plot A (3.6, Cl: 2.9-4.4) compared to plot $\mathrm{B}(7.3, \mathrm{Cl}: 5.9-8.9, \mathrm{P}<0.0001$, Figure $2 \mathrm{D})$. The difference in larval tick burden between plots was affected by week $(P=0.0174)$, indicating that the slope of the larval tick burden differed between plots in the middle of the trapping period (data not shown). Larval tick burden was positively correlated to rodent weight $(P<0.0001$, Figure 6$)$. With each increasing gram of body weight, larval tick burden was multiplied by 1.06 . Larval tick burden was affected by week $(P<0.0001)$, indicating that the line was not horizontal in the middle of the trapping period, and week $^{2}(\mathrm{P}<0.0001)$, indicating a part of a parabolic curve in tick burden throughout the trapping period (Figure 4). Nymphal tick burden differed between years and was higher in $2013(0.11$, Cl: 0.05-0.25) compared to 2014 (0.03, Cl: 0.02-0.05, P=0.0015, Figure 7A). Nymphal tick burden also differed between rodent species and was lower on bank voles $(0.04, \mathrm{Cl}$ : $0.02-$ 0.07 ) compared to wood mice $(0.09, \mathrm{Cl}: 0.05-0.18, \mathrm{P}=0.0081$, Figure $7 \mathrm{~B})$. 


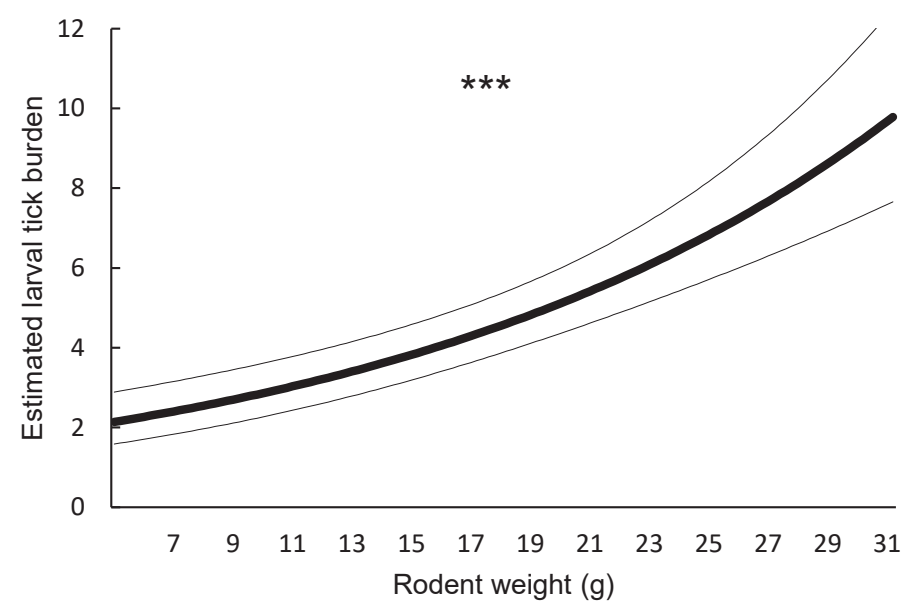

Figure 6. Estimated larval tick burden on rodents in week 32 by rodent weight. Thin lines represent upper and lower bounds of the $95 \% \mathrm{Cl}$. Significant indications are illustrated as ${ }^{* * *}=\mathrm{P}<0.001$.
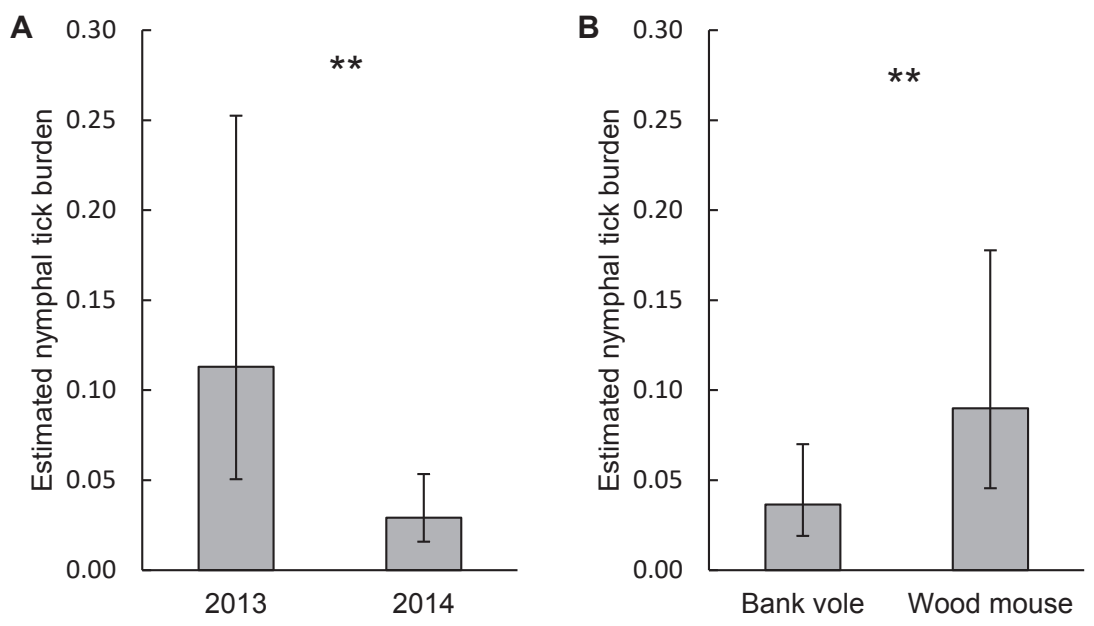

Figure 7. Estimated nymphal tick burden on rodents in week 32 per (A) year and (B) rodent species. Error bars represent $95 \% \mathrm{Cl}$. Significant indications are illustrated as ${ }^{* *}=\mathrm{P}<0.01$. 
The difference in nymphal tick burden between rodent sexes differed between years $(P=0.0203)$; in 2013 nymphal tick burden was higher on males compared to females $(P=0.0149)$, whereas rodent sex had no effect on nymphal tick burden in $2014(P=0.6634$, Figure 8). Nymphal tick burden was positively correlated to rodent weight ( $P=0.0004$, Figure 9). With each gram of body weight, nymphal tick burden was multiplied by 1.19 . The difference in nymphal tick burden between plots was affected by week $(P=0.0414)$, indicating that the slope of the nymphal tick burden differed between plots in the middle of the trapping period (data not shown).

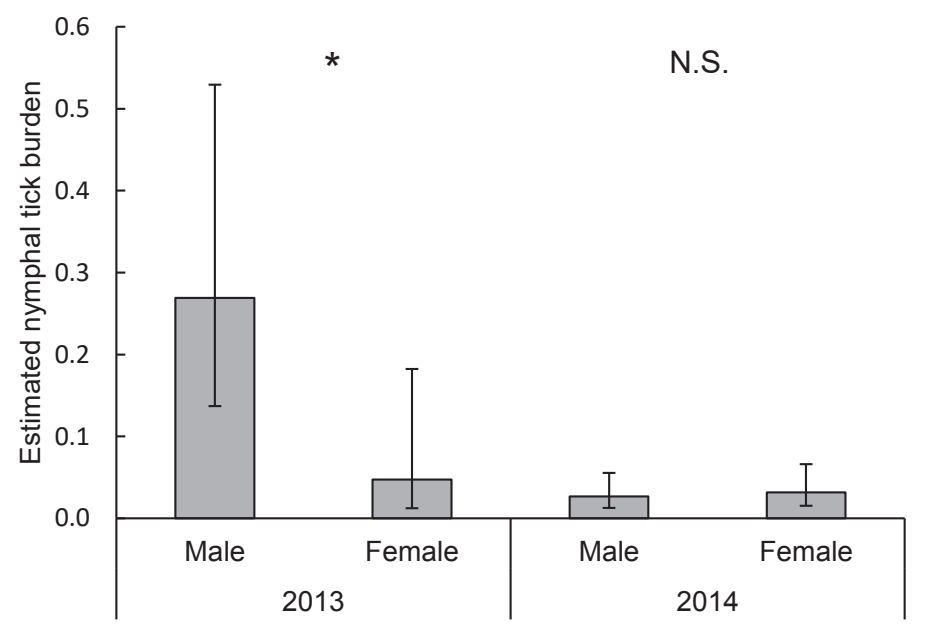

Figure 8. Estimated nymphal tick burden on rodents in week 32 per rodent sex per year. Error bars represent $95 \%$ Cl. Significant indications are illustrated as * $=P<0.05$.

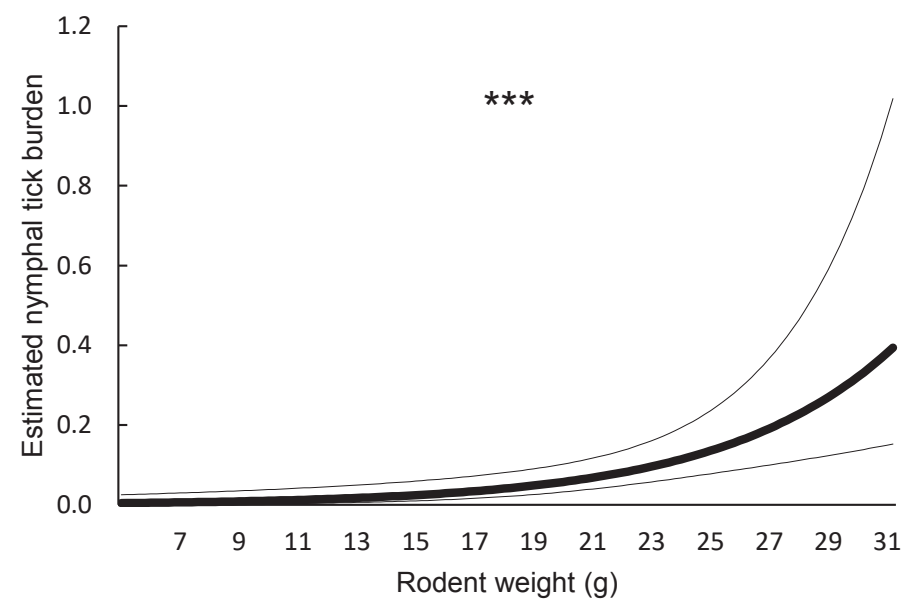

Figure 9. Estimated nymphal tick burden on rodents in week 32 by rodent weight. Thin lines represent upper and lower bounds of the $95 \% \mathrm{Cl}$. Significant indications are illustrated as ${ }^{* *}=\mathrm{P}<0.001$. 


\section{Rodent infection rate}

In 2013, 60 ear biopsies were analysed for infection, from which 21 (35\%) were infected with B. burgdorferi s.I. and 3 (5\%) were infected with B. miyamotoi (Table 2). In 2014, 705 ear biopsies were analysed for infection, from which 100 (14\%) were infected with $B$. burgdorferi s.l. and $8(1 \%)$ were infected with $B$. miyamotoi.

Rodent infection rate with $B$. burgdorferi s.l., differed between years and was higher in 2013 (0.68, Cl: 0.45-0.85) compared to 2014 (0.16, Cl: 0.12-0.22, $\mathrm{P}<0.0001$, Figure 10). The difference in rodent infection rate between years was affected by week ${ }^{2}(P=0.0190)$, indicating that it differed throughout the trapping period (Figure 11). There was a shorter, but higher peak in 2013 compared to 2014 . Week ${ }^{2}$ also affected rodent infection rate by itself $(P=0.0034$, Figure 11 ); it peaked in summer compared to spring and autumn.

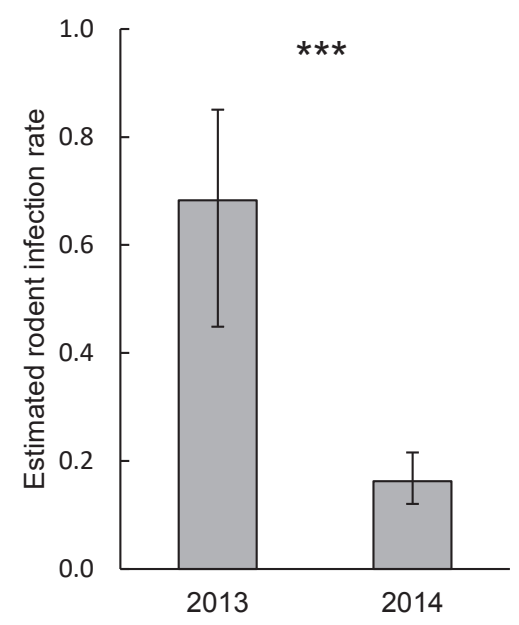

Figure 10. Estimated infection rate of rodents with B. burgdorferi s.I. in week 32 per year. Error bars represent $95 \%$ Cl. Significant indications are illustrated as ${ }^{* *}=\mathrm{P}<0.001$. 


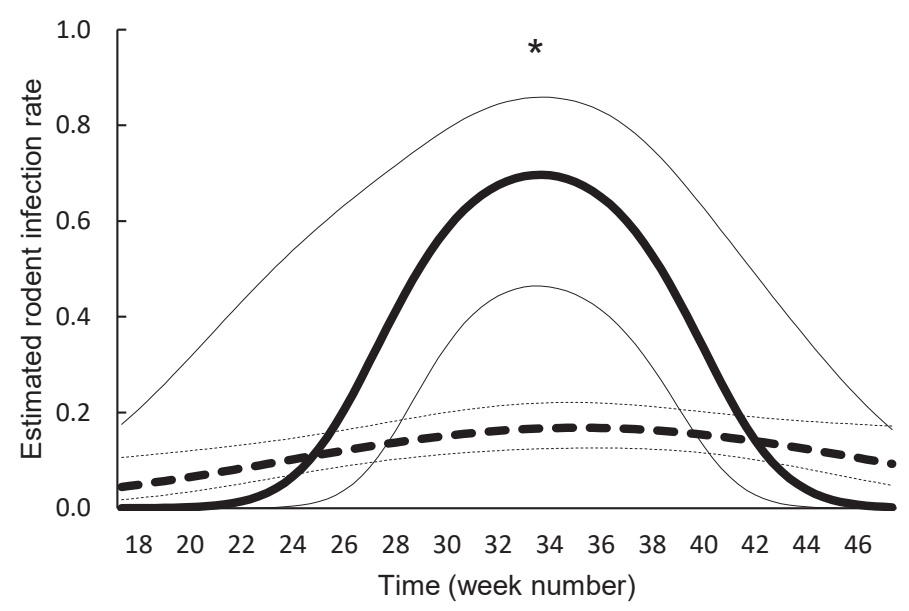

Figure 11. Estimated rodent infection rate in 2013 (solid line) and 2014 (dashed line) over time. Thin lines represent upper and lower bounds of the $95 \% \mathrm{Cl}$. Significant indications are illustrated as ${ }^{*}=\mathrm{P}<0.05$.

Rodent infection rate increased with increasing rodent weight $(P=0.0011$, Figure 12). For each increasing gram of body weight, rodent infection rate was multiplied by 1.09 . The effect of rodent sex on rodent infection rate was affected by week $(P=0.0218)$, indicating that the slopes of the lines differed at the middle of the trapping period, and week $^{2}(\mathrm{P}=0.0087)$, indicating that it differed throughout the trapping period (Figure 13). Rodent infection rate was highest in weeks 32-36 for both sexes.

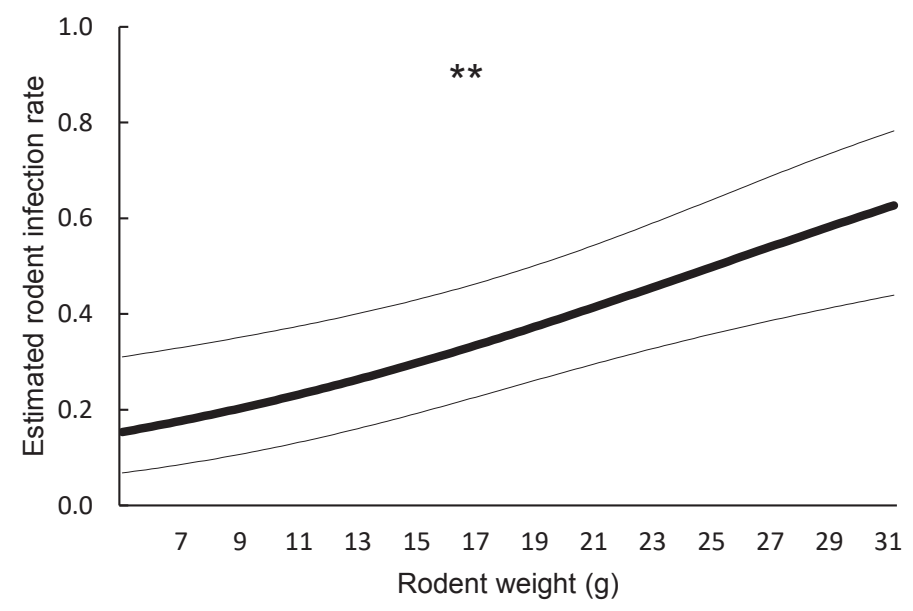

Figure 12. Estimated rodent infection rate in week 32 by rodent weight. Thin lines represent upper and lower bounds of the $95 \% \mathrm{Cl}$. Significant indications are illustrated as ** $=\mathrm{P}<0.01$. 


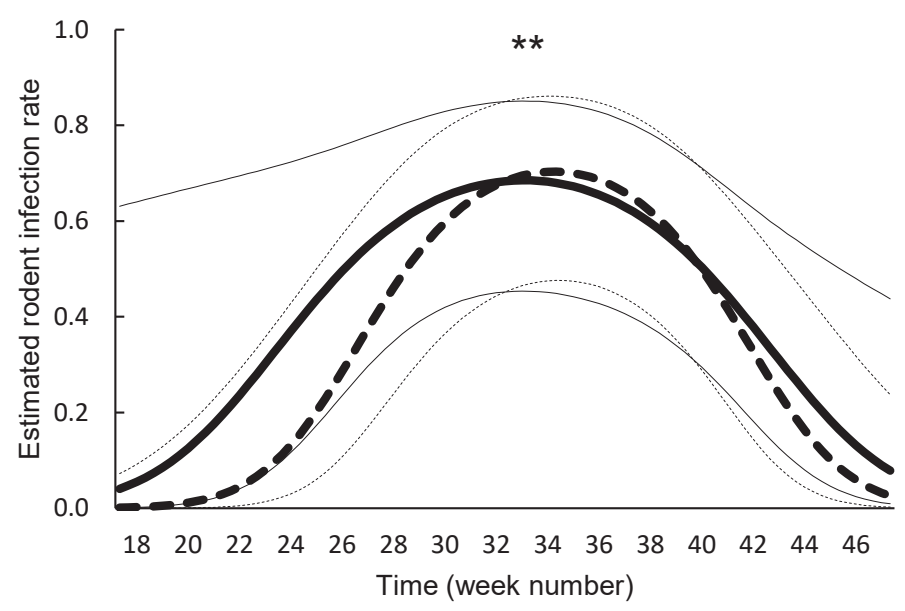

Figure 13. Estimated rodent infection rate of male (solid line) and female (dashes line) rodents over time. Thin lines represent upper and lower bounds of the $95 \% \mathrm{Cl}$. Significant indications are illustrated as ** $=\mathrm{P}<0.01$.

\section{Rodent infectivity}

In 2013, 496 larvae from B. burgdorferi s.l.-infected rodents were analysed for infection, from which 228 (46\%) were infected with B. burgdorferi s.l. (Table 2). In 2013, 22 larvae from B. miyamotoi-infected rodents were analysed for infection, from which $7(32 \%)$ were infected with B. miyamotoi. In 2014, 246 larvae from B. burgdorferi s.l.-infected rodents were analysed for infection, from which 94 (38\%) were infected with B. burgdorferi s.I. In 2014, 8 larvae from B. miyamotoi-infected rodents were analysed for infection, from which $3(38 \%)$ were infected with B. miyamotoi. For B. burgdorferi s.I., estimated rodent infectivity was 0.38 (Cl: $0.30-0.47)$ and was not affected by any of the main factors or two-factor interactions $\mathrm{P}>0.05$ (Appendix 2).

\section{Moulting success of larvae}

In 2013, 1,128 engorged larvae were collected from the male rodents in the laboratory, from which 1,086 (96\%) moulted into nymphs (Table 2). In 2014, 1,410 engorged larvae were collected from the male rodents in the laboratory, from which 1,349 (96\%) moulted into nymphs. Moulting success differed between plots and was lower for the larvae that fed on the rodents in plot $\mathrm{A}(0.91, \mathrm{Cl}: 0.88-0.94)$ compared to the larvae that fed on the rodents in plot $\mathrm{B}$ (0.95 Cl: 0.93-0.96, $P=0.0437$, Figure 14). Moulting success did not differ between rodent species $(P=0.0567)$ but the effect of rodent species was affected by year $(P=0.0182)$; in 2013 moulting success was not affected by rodent species $(P=0.7354)$ but in 2014 moulting success was higher for larvae that fed on bank voles compared to larvae that fed on wood mice 
$(P=0.0016$, Figure 15). Moulting success of larvae was affected by week $(P<0.0001)$, indicating that the slope of moulting success was not horizontal in the middle of the trapping period (data not shown).

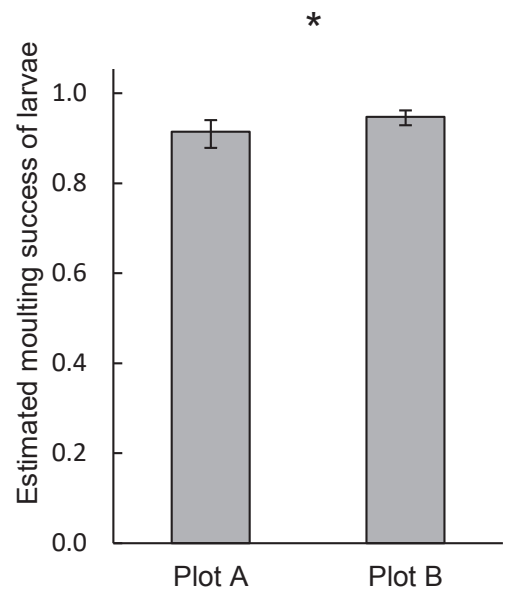

Figure 14. Estimated moulting success of larvae per plot. Error bars represent $95 \% \mathrm{Cl}$. Significant indications are illustrated as ${ }^{*}=P<0.05$.

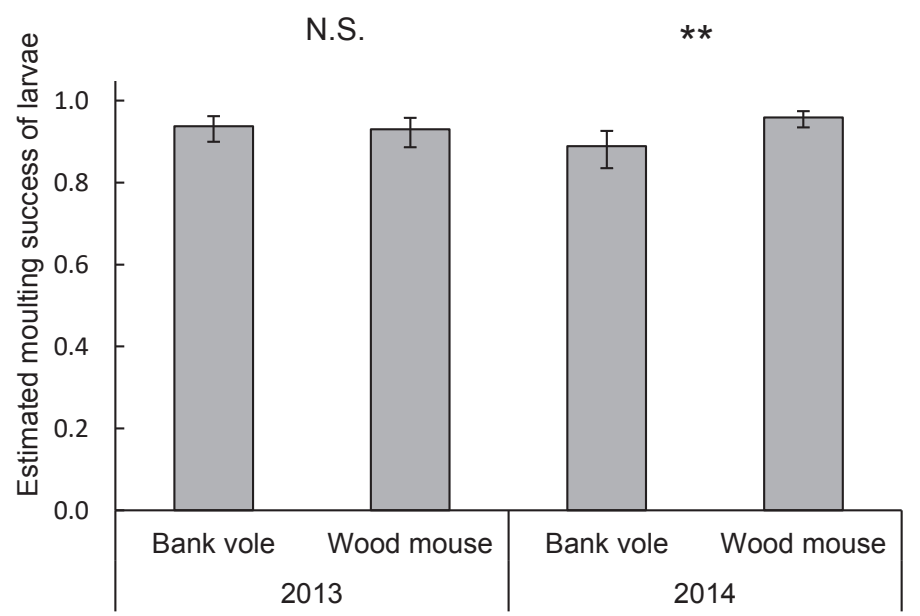

Figure 15. Estimated moulting success of larvae per rodent species per year. Error bars represent $95 \% \mathrm{Cl}$. Significant indications are illustrated as ${ }^{*}=\mathrm{P}<0.05$. 


\section{Contribution of rodents to the density of infected nymphs}

The calculated relative numbers of infected questing nymphs per year, rodent species, plot and rodent sex are shown in table 2. The relative number of $B$. burgdorferi s.l.-infected questing nymphs that emerged from the larvae that fed on the rodents was 0.058 for 2013 and 0.081 for 2014. The relative number of $B$. miyamotoi-infected questing nymphs that emerged from the larvae that fed on the rodents was the same for 2013 and 2014 (0.00006).

\section{Discussion}

Bank voles and wood mice were the only rodents species trapped in this study and are the most common rodent species in The Netherlands (de Boer et al. 1993a, Gassner et al. 2008). Rodent density was about one order of magnitude lower in 2013 than in 2014. The contribution of the 2013 rodent population to the density of $B$. burgdorferi s.l.-infected nymphs was, however, only a little less than that of the 2014 population. The contribution to the density of B. miyamotoi-infected nymphs did not differ between years.

Although the total number of larvae that fed on the rodent population was higher in 2014 , the estimated larval and nymphal tick burdens were lower compared to 2013. These lower tick burdens caused that rodent infection rate with B. burgdorferi s.l. decreased in 2014 . As a result the contribution of the 2014 population to the density of $B$. burgdorferi s.l. infected nymphs increased only slightly. The lower mean larval tick burden in 2014 was probably due to a dilution effect caused by the high rodent density (Krasnov et al. 2007, Rosa et al. 2007, Kiffner et al. 2011). Rodent density is largely affected by food availability, which also affects home range size (Stradiotto et al. 2009). A large home range size of rodents can result in more tick encounters and therefore, a higher tick burden (Sonenshine and Stout 1968, Devevey and Brisson 2012). The contribution of the rodent populations to the density of B. miyamotoiinfected nymphs did not differ between years, supporting the importance of transovarial transmission for this spirochete (Krause et al. 2015).

We found that larval and nymphal tick burdens were higher on wood mice compared to bank voles, which is supported by previous studies from different ecological settings (Tälleklint and Jaenson 1997, Mysterud et al. 2015). In addition, more larvae were found on male rodents compared to female rodents and rodents with a large body weight, which are in general older compared to smaller-sized ones, which is also supported by previous studies from different ecological settings (Sonenshine and Stout 1968, Nilsson and Lundqvist 1978, Tälleklint and Jaenson 1997, Devevey and Brisson 2012). The higher tick burdens on males compared to females may be the effect of larger home ranges of males (Stradiotto et al. 2009) and therefore tick encounter rates. We also found a remarkable variation in larval tick burden between plots with comparable vegetation types but at a small geographical scale. This may perhaps have 
been caused by a difference in roe deer density between plots, affecting densities of questing ticks by affecting host finding success of adult ticks (Gray et al. 1992, Ostfeld et al. 2006). The effect of season on larval tick burden differed between rodent species and was larger in wood mice compared to bank voles. This may be the result of different ecological characteristics of the rodents. E.g. wood mice are more active at night, whereas bank voles are more active during the day, a diel pattern of questing activity of larvae, affected by climatic conditions, could result in a stronger effect of season on larval tick burdens on wood mice, because climatic conditions may be more stable in spring and autumn.

Larval and nymphal tick burdens were higher in 2013 compared to 2014. Considering Lyme borreliosis risk, only infected rodents can infect feeding larvae. Rodents can become infected with Borrelia burgdorferi s.I. through the bite of an infected larva or nymph (Radolf et al. 2012, Van Duijvendijk et al. 2016). A high tick burden on rodents, therefore, results in a high exposure to the spirochaetes. As a result of this higher tick burden, rodent infection rate with $B$. burgdorferi s.l. was higher in 2013 compared to 2014. This lower rodent infection rate in 2014 contributed to a small increase in the density of $B$. burgdorferi s.l.-infected nymphs fed by the 2014 rodent population compared to the 2013 rodent population. Variation in rodent infection rate between years was also found by Kurtenbach et al. (1995) and is most likely caused by the annual variations in the ratio rodent/questing tick density, with a dilution effect at high rodent densities, leading to lower $B$. burgdorferi s.I. infections in rodents. In our study, rodent infection rate peaked during summer, which would increase the infection rate in larvae that feed on the rodent population. An increase in rodent infection rate in spring was also found by Kurtenbach et al. (1995). These authors did, however, not find a decrease in the infection rate of feeding larvae in the autumn. This difference may have been caused by a difference in longevity of the rodents. In our study, rodent infection rate showed a similar peak over time as larval tick burden. Larval tick burden is likely to be affected by larval questing activity. Likewise, tick questing activity affected rodent infection rate in white footed mice (Hofmeister et al. 1999), which was probably caused by an increased tick burden on rodents. We did not find a difference in rodent infection rate between rodent species, which is in contradiction to the results of Kybicova et al. (2008) and Humair et al. (1999), who found a higher infection rate in bank voles compared to wood mice. It is unclear what caused this difference. Our molecular analysis did not distinguish between the $B$. burgdorferi s.l. genospecies, but previous research showed that rodents and larvae collected from rodents are mainly infected with $B$. afzelii (Humair et al. 1999, Hanincová et al. 2003a, Kybicova et al. 2008), which can cause skin problems in humans (Strle and Stanek 2009, Stanek et al. 2012). For the infected rodents to have an effect on the density of infected nymphs, the spirochaetes should also be transmitted to feeding larvae. Rodent infectivity was 0.43 based on the raw data, and estimated rodent infectivity was 0.38 when corrected for trap number and rodent number. These values are in 
line with the findings of Burri et al. (2014). Rodent infectivity was not affected by any of the measured variables. This is in contradiction with the results of Perez et al. (2012) and Humair et al. (1999), who found a higher infectivity of bank voles than of wood mice. Infected rodents do not lose their infection, but the infectivity varies between individual rodents and was enhanced by successive infestations with larval ticks (Gern et al. 1994).

Overall, moulting success of larvae was always $>90 \%$ and was not affected by rodent species. This is in line with the findings of Tälleklint and Jaenson (1997), but in contradiction to the results of Humair et al. (1999), who found a higher moulting rate of ticks that fed on wood mice (33.3\%) compared to bank voles $(6.2 \%)$. We found that the effect of rodent species varied between years. Dizij and Kurtenbach (1995) showed that moulting success of larvae that fed on bank voles decreased with tick successive infestations, whereas this was not found for larvae feeding on wood mice. Years with high tick burdens could, therefore, result in reduced moulting success for ticks that fed on bank voles, possibly caused by immunity to tick antigens. We found, however, that moulting success was significantly reduced in bank voles in 2014 , when tick burdens were low.

Understanding the factors that cause the aggregation of ticks on rodents helps understanding the transmission dynamics of $B$. afzelii and identify the $20 \%$ of the rodents that contribute to $80 \%$ of the $B$. afzelii transmission cycle (Woolhouse et al. 1997, Lloyd-Smith et al. 2005, Stein 2011). Larval density, and not rodent density was found to be the best explanatory variable for nymphal density in the following year (Rosa et al. 2007).

Larval tick burdens on rodents show high temporal variation within and between years. A peak in rodent density (e.g. after a mast year) results in reduced tick burdens on rodents and a reduced rodent infection rate. Therefore, we conclude that years with high rodent densities do not lead to an increase in the contribution of the rodent population to the density of $B$. burgdorferi s.l.-infected nymphs and B. miyamotoi-infected nymphs. The risks for Lyme borreliosis and Borrelia miyamotoi disease are, therefore, not directly affected by a temporal variation in rodent density.

\section{Acknowledgements}

We thank everybody who helped with the rodent trappings and staatsbosbeheer for providing permission to conduct the experiment in their forest. 



\title{
Chapter 5
}

\section{A Borrelia afzelii infection increases larval tick burden on}

\author{
Myodes glareolus and nymphal \\ body weight of Ixodes ricinus
}

Gilian van Duijvendijk, Wouter van Andel, Manoj Fonville, Gerrit Gort, Joppe W. Hovius, Hein Sprong, Willem Takken 


\section{Abstract}

Several micro-organisms have been shown to manipulate their host or vector to enhance their own transmission. Here we examined whether an infection with Borrelia afzelii affects its transmission between its bank vole (Myodes glareolus) host and tick vector.

Captive-bred bank voles were inoculated with $B$. afzelii or control medium, after which host preference of $I$. ricinus nymphs was determined in a Y-tube olfactometer. Thereafter, infected and uninfected bank voles were placed in a semi-field arena containing questing larvae to measure larval tick attachment. Engorged larvae were collected from these bank voles, moulted into nymphs, weighed and analysed for infection by PCR.

Nymphs were attracted to the odours of a bank vole compared to ambient air and preferred the odours of an infected bank vole over that of an uninfected bank vole. In the semi field arena, infected male bank voles had greater larval tick burdens then uninfected males, while similar larval tick burdens were observed on females regardless of infection status. Nymphal ticks that acquired a $B$. afzelii infection had higher body weight than nymphs that did not acquire an infection regardless of the infection status of the vole. These results show that $B$. afzelii infection in bank voles increases larval tick burden and that a $B$. afzelii infection in larvae increases nymphal body weight. This finding provides novel ecological insights into the enzootic cycle of $B$. afzelii. 


\section{Introduction}

Borrelia afzelii can cause Lyme borreliosis in humans and cycles from Ixodes ricinus larvae or nymphs to rodents, which in turn can infect feeding larvae (Kurtenbach et al. 1998b, Humair et al. 1999, Hanincová et al. 2003a, Kybicova et al. 2008, Van Duijvendijk et al. 2016). The number of larvae feeding on an infected rodent, therefore, affects $B$. afzelii infection prevalence in questing nymphs. Aggregation of parasites on hosts often follows the Pareto principle, commonly referred to as the $80-20$ rule (Woolhouse et al. 1997). This principle implies that parasites are not equally distributed amongst hosts and has been described for the natural parasitism of I. ricinus on rodents (Tälleklint and Jaenson 1997, Randolph et al. 1999). This principle also implies that very few rodents contribute to the vectors' lifecycle and subsequently the enzootic cycle of vector pathogens in nature (Randolph et al. 1999, Perkins et al. 2003, Brunner and Ostfeld 2008). Many factors are suggested to play a role in this heterogeneity of tick burden, like the heterogeneous dispersal of larvae and rodents in the environment, rodent sex, rodent age, immune response, genetic differences of host susceptibility, tick behaviour and host behaviour (Kaufman 1989, De Boer et al. 1993b, Craine et al. 1995, Wikel 1996, Schmidt et al. 1999, Hughes and Randolph 2001, Shaw et al. 2003, Keesing et al. 2009, Harrison et al. 2010, Pisanu et al. 2010, Devevey and Brisson 2012, Dallas and Fore 2013, Taylor et al. 2013b).

Several vector-borne pathogens affect the odour of their vertebrate host to increase attractiveness for their vectors. This was shown for humans and birds infected with Plasmodium, which resulted in attraction of more mosquitoes (Lacroix et al. 2005, Cornet et al. 2013) and hamsters infected with Leishmania attracting more sandflies (O'Shea et al. 2002). It is know that parasitism of rodents increases energy demand (Demas et al. 1997, Schwanz et al. 2011a), which increases foraging time and food uptake (Scantlebury et al. 2007). This would subsequently increase encounter rates with ticks and their pathogens. Whether $B$. afzelii infection compounds the effect of parasitism on rodents is unknown. Infection with tick-borne encephalitis virus, however, has been show to increase the attractiveness of male rodents to oestrus females and increase aggressiveness in social conflict (Moshkin et al. 2002), resulting in larger home range size and increasing potential for viral transmission by sexual activity and ticks.

In addition to affecting host behavior, several pathogens have been shown to affect their arthropod vectors, resulting in increased transmission (Killick Kendrick et al. 1977, Randolph 1991, Hu et al. 1997b, Koella et al. 1998, Hurd 2003, Lefèvre and Thomas 2008, Van Houte et al. 2013). There is also little, but inconclusive evidence that a B. burgdorferi s.l. infection can increase, blood meal size, nymphal body weight, nymphal survival and host-finding efficacy of I. ricinus (Faulde and Robbins 2008, Gassner 2010, Herrmann and Gern 2010, 2012, 
Herrmann et al. 2013b). An enhanced survival increases the chance that a nymph finds a host and transmits the spirochaetes to a rodent. These studies were performed, however, on fieldcollected rodents and ticks and, therefore, differences in other life history traits, like species or age of the hosts that were fed upon by the ticks during their larval stage, may have influenced the results.

The aim of the present study was to determine whether a $B$. afzelii infection enhances its transmission between bank voles and I. ricinus.

\section{Materials and Methods}

\section{Study organisms.}

Questing Ixodes ricinus were collected by dragging a $1 \mathrm{~m}^{2}$ white cotton blanket over low vegetation and leaf litter in two forests near Wageningen, The Netherlands $\left(52^{\circ} 01^{\prime} 36^{\prime \prime} \mathrm{N}\right.$, $5^{\circ} 41^{\prime} 21^{\prime \prime} \mathrm{E}$ and $\left.52^{\circ} 02^{\prime} 29^{\prime \prime} \mathrm{N}, 5^{\circ} 44^{\prime} 50^{\prime \prime} \mathrm{E}\right)$. Attached larvae and nymphs were collected in $50 \mathrm{ml}$ tubes connected to an aspirator and were housed at room temperature, $90 \%$ relative humidity and a natural photoperiod until further use.

Bank voles (Myodes glareolus) were live trapped and bred in the laboratory. Seventy four, two to four month old bank voles, which had never been exposed to ticks and/or B. burgdorferi s.I. were used. Twenty-nine bank voles were inoculated subcutaneously in the neck with $5^{\star} 10^{6} B$.

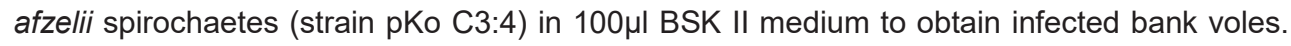
Another twenty-nine bank voles were inoculated with $100 \mu \mathrm{l}$ of BSK II medium without spirochaetes to obtain uninfected bank voles. Sixteen bank voles were not inoculated and termed naive bank voles and used as controls in the Y-tube olfactometer (see below). Two weeks after inoculation a small ear biopsy was collected using sterile tweezers and scissors and stored in $70 \%$ alcohol at $-20^{\circ} \mathrm{C}$ until analysis. Infection status of infected bank voles and uninfected bank voles was confirmed by PCR (see below). Experiments were conducted 2-4 weeks after inoculation of the bank voles. Pairs of bank voles that were tested were of equal sex and from the same litter. All experiments were approved by the Ethical committee of Wageningen University (permit number 2012065).

\section{Effect of rodent odour and a $B$. afzelii infection in rodents on tick behavior.}

A closed-system Y-tube olfactometer (Takabayashi and Dicke 1992) was used to analyse the effects of bank vole odour and a $B$. afzelii infection in bank voles on tick behavior. Pressurized air was split in two streams, which were led through two 5 I glass animal holding chambers. These chambers contained no odour source, a naive bank vole, an uninfected bank vole or an 
infected bank vole. Four combinations (treatments) were tested (Figure 1). Bank voles were placed in the animal holding chambers $10 \mathrm{~min}$. before the start of the experiment to acclimatize and to allow for their odours to spread through the chambers. After the animal holding chamber, each stream of air was split again in two streams and was controlled for flowrates of 1.2 liter/min (Sho-rate rotameter, Brooks Instrument B.V.), humidified (>90\% relative humidity, by leading the air through 1 I bottles filled with water) and led to the upper ends of two glass Y-tube olfactometers (arms: $7 \mathrm{~cm}$, stem: $8 \mathrm{~cm}$, diameter: $8 \mathrm{~mm}$ ). These Y-tubes were positioned at an upward angle of $45^{\circ}$. Per treatment, 32 replicates of 10 nymphs were released at the down-wind end of the Y-tube olfactometer and their positions ("left", "right", or "no choice" when in the stem of the $Y$-tube) were recorded after $15 \mathrm{~min}$. Each nymph was tested only once. Odour sources were switched between the Y-tube arms and all materials were cleaned with $70 \%$ alcohol between replicates. The experiment was conducted at $25 \pm 2{ }^{\circ} \mathrm{C}$ and $740 \mathrm{Lux}$.

\section{Effect of a $B$. afzelii infection in rodents on acquired tick burden.}

The effect of a $B$. afzelii infection in bank voles on acquired tick burden was tested in a semifield arena of $120 \times 80 \times 80 \mathrm{~cm}$ filled with 40 I of humid potting soil and a $3 \mathrm{~cm}$ thick layer of autoclaved oak and beech leaves. Before the start, approximately 1,000 I. ricinus larvae were distributed throughout the arena. A heat cable was attached to the upper inside of the arena to prevent larvae from escaping. Grain and carrot were provided ad libitum. One bank vole pair, consisting of an infected and an uninfected animal, was allowed to move freely in the arena for $24 \mathrm{~h}$, after which both bank voles were trapped and housed individually in Makrolon type II cages over pans with water with petroleum jelly on the edge. Engorged larvae were collected from the water after three and seven days and the total number of larvae per bank vole was recorded as a measurement for tick burden. Twenty nine bank vole pairs were tested and each pair was used only once. The experiment was conducted at $20-25^{\circ} \mathrm{C}$. After seven days, the bank voles were weighed and euthanized by cervical dislocation.

\section{Effect of a $B$. afzelii infection on nymphal body weight.}

Engorged larvae that were collected from the water-filled pans were stored individually in ventilated $0.2 \mathrm{ml}$ Eppendorf tubes at $20^{\circ} \mathrm{C}$ and $>90 \%$ relative humidity. A maximum of four emerged nymphs were randomly selected per bank vole and weighed on a microbalance to the nearest microgram after 3-4 months. These nymphs were thereafter stored at $-20^{\circ} \mathrm{C}$ until analysis for $B$. afzelii infection. Ticks were divided in three groups; 1 ) infected ticks that fed on infected bank voles, 2) uninfected ticks that fed on infected bank voles and 3) uninfected ticks that fed on uninfected bank voles. 


\section{DNA extraction and PCR amplification.}

Ear biopsies and nymphs were analysed individually. Nymphs were heated for $20 \mathrm{~min}$ at $99^{\circ} \mathrm{C}$ in $100 \mu \mathrm{l}$ ammonium hydroxide $(\mathrm{NH} 4 \mathrm{OH}, 1 \mathrm{M})$, after which they were centrifuged and heated for $20 \mathrm{~min}$ at $99^{\circ} \mathrm{C}$ with open lids to evaporate the ammonia. The lysates were stored at $4^{\circ} \mathrm{C}$. DNA was extracted from the ear biopsies using the Qiagen DNeasy Blood \& Tissue Kit (Jahfari et al. 2014). Borrelia burgdorferi s.I. DNA was detected using $\mathrm{PPCR}$ in the IQ Multiplex Powermix with a volume of $20 \mu \mathrm{l}$, containing iTaq DNA polymerase (Bio-Rad Laboratories, USA), $200 \mathrm{nM}$ of each primer and $5 \mu \mathrm{l}$ of template DNA (Heylen et al. 2013b). Outer surface protein A gene (OspA) (forward primer: 5'-AAT ATT TAT TGG GAA TAG GTC TAA-3'; reverse primer: 5'-CTTTGT CTT TTT CTT TRC TTA CA-3' and probe: 5'-Atto520-AAG CAA AAT GTT AGC AGC CTT GA-BHQ1-3') and the $B$. burgdorferi s.l. flagellin gene (flaB) (forward primer: 5'-CAG AIA GAG GTT CTA TAC AIA TTG AIA TAG A-3'; reverse primer: 5'-GTG CAT TTG GTT AIA TTG YGC-3' and probe: 5'-Atto425-CAA CTI ACA GAI GAA AXT AAI AGA ATT GCT GAI CA-Pho-3', where $X$ stands for an internal BHQ-1 quencher attached to thymine) were used as targets. The qPCR cycling program (using a light cycler 480 real-time PCR system, Hoffmann-La Roche, Switzerland) was performed using a two-step PCR program: Taq activation for $5 \mathrm{~min}$ at $95^{\circ} \mathrm{C}$ followed by 60 cycles of $5 \mathrm{~s}$ at $94^{\circ} \mathrm{C}$ and $35 \mathrm{~s}$ at $60^{\circ} \mathrm{C}$ involving a single point measurement at $60^{\circ} \mathrm{C}$ with corresponding filters, finishing with one cycle of $20 \mathrm{~s}$ at $37^{\circ} \mathrm{C}$. Validation of runs included checking and verifying controls, amplification curves and fluorescence scale and analysis was performed using a second derivative calculation.

\section{Data analysis.}

Effect of bank vole odour and a $B$. afzelii infection in bank voles on tick behavior (number of nymphs in one arm given the total number of responding nymphs in both arms of the $\mathrm{Y}$-tube olfactometer) was analysed per treatment using a GLM (PROC GENMOD), assuming a binomial distribution with logit link function. Bank vole sex had no effect and was excluded from the model. Effect of a $B$. afzelii infection in bank voles on acquired tick burden (number of larvae on the infected bank vole, given the total number of larvae on both bank voles) was analysed using a GLM (PROC GENMOD), assuming a binomial distribution with logit link function with bank vole sex as factor and difference in bank vole body weight as covariate. An extra scale parameter based on Pearson's chi-square was used to correct for overdispersion. The effect of a $B$. afzelii infection on nymphal body weight was analysed using a mixed model (PROC MIXED) with random effects of mouse (on which the ticks fed as larvae) and fixed effects of group and weighing date. Tick burden had no effect on nymphal body weight and was excluded from the model. To analyse the effect of a $B$. afzelii infection in ticks (that had fed on infected bank voles) on nymphal body weight, groups 1 and 2 were compared (see 
above). To analyse the effect of a $B$. afzelii infection in the bank voles on the body weight of nymphs, groups 2 and 3 were compared. Effects were considered significant at $P<0.05$. All analysis were performed with SAS statistical software (SAS Institute Inc. (SAS 2004)), version 9.3.

\section{Results}

\section{Effect of bank vole odour and a $B$. afzelii infection in bank voles on tick behavior.}

In the Y-tube-olfactometer nymphs showed no preference to either stream with ambient air $(P=0.853$, Figure 1$)$ or to either stream with naïve bank vole odour $(P=0.379)$. Significantly more nymphs chose bank vole odour compared to ambient air $(80.4 \%$ and $19.6 \%$ of the responding nymphs, respectively, $\mathrm{P}<0.001)$. Nymphs also demonstrated a significant preference for the odour of a $B$. afzelii-infected bank vole compared to the odour of an uninfected bank vole ( $66 \%$ and $34 \%$ of the responding nymphs, respectively, $\mathrm{P}=0.022)$.

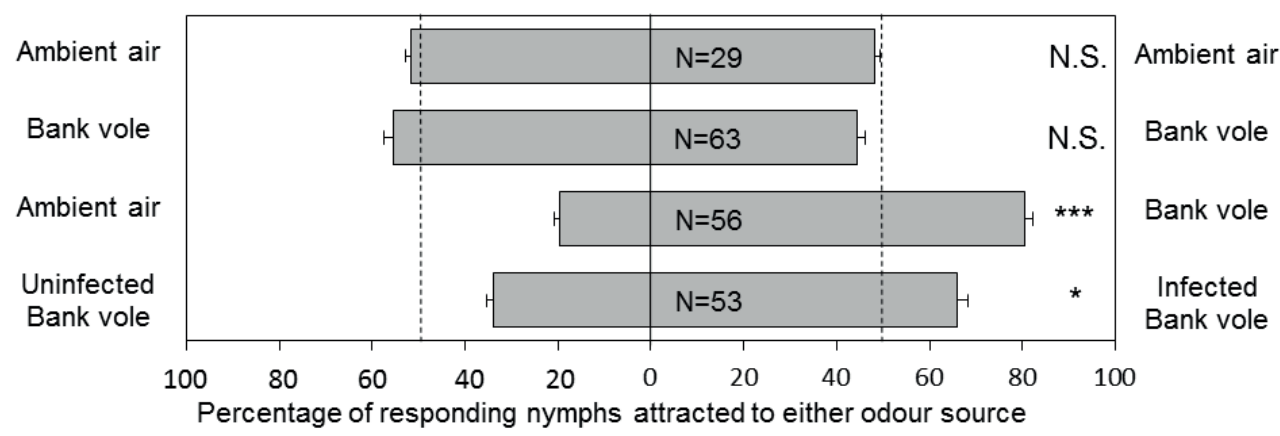

Figure 1. Percentages of the responding $I$. ricinus nymphs that were attracted to different odour sources in a closedsystem Y-tube olfactometer. Numbers inside bars represent the number of nymphs that responded. Error bars represent S.E. of percentages of ticks that chose for either odour source, based on 32 replicates of 10 nymphs. Significance indications represent choice differences within treatments, illustrated as * $P<0.05$, ${ }^{* *} P<0.001, N . S$. = not significant.

\section{Effect of a $B$. afzelii infection in bank voles on acquired tick burden.}

In the semi-field arena, one pair of bank voles did not acquire any larva and was excluded from the analysis. The effect of infection status on larval tick burden differed between bank vole males and females $(P=0.011)$. Infected male bank voles acquired significantly greater larval tick burdens $(46.3 \pm 14.9)$ compared to uninfected male bank voles $(28.9 \pm 6.5, P<0.001$, 
Figure 2). In females, larval tick burden did not differ between infected bank voles $(23.1 \pm 11.4)$ and uninfected bank voles $(21.3 \pm 5.0, \mathrm{P}=0.780)$.

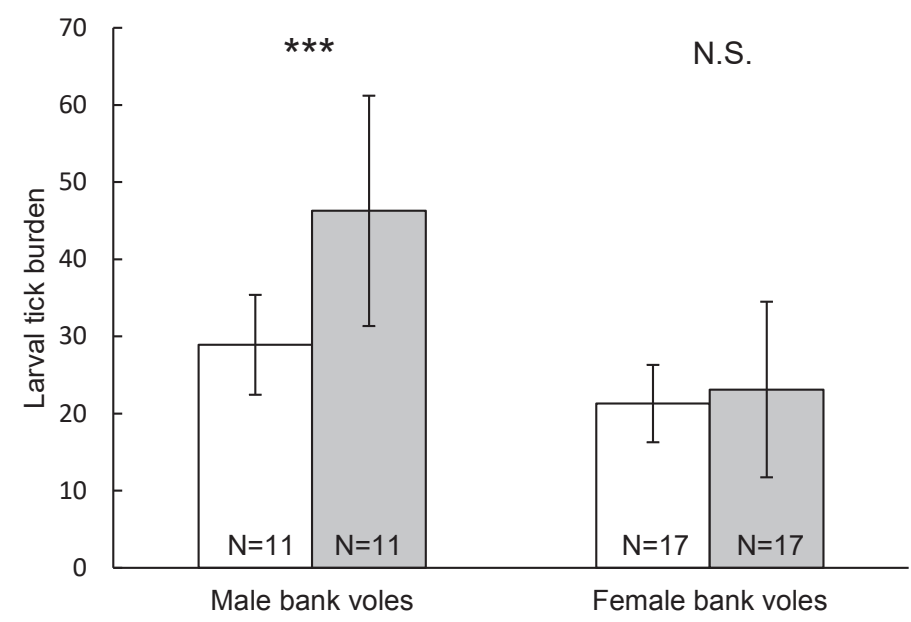

Figure 2. Average acquired larval tick burden ( \pm S.E.) of uninfected bank voles (white bars) and bank voles infected with $B$. afzelii (grey bars) after $24 \mathrm{~h}$ in a semi-field arena with questing larvae. $\mathrm{N}=$ number of bank voles. Significance indications are illustrated as ${ }^{* * *} \mathrm{P}<0.001$, N.S. $=$ not significant.

\section{Effect of a $B$. afzelii infection on nymphal body weight.}

Infected nymphs from infected bank voles were heavier $(0.198 \pm 0.005 \mathrm{mg})$ compared to uninfected nymphs from infected bank voles $(0.180 \pm 0.003 \mathrm{mg}, \mathrm{P}=0.023$, Figure 3$)$. Body weight did not differ between uninfected nymphs from infected bank voles $(0.180 \pm 0.003 \mathrm{mg})$ and uninfected nymphs from uninfected bank voles $(0.182 \pm 0.003 \mathrm{mg}, \mathrm{P}=0.995)$. 


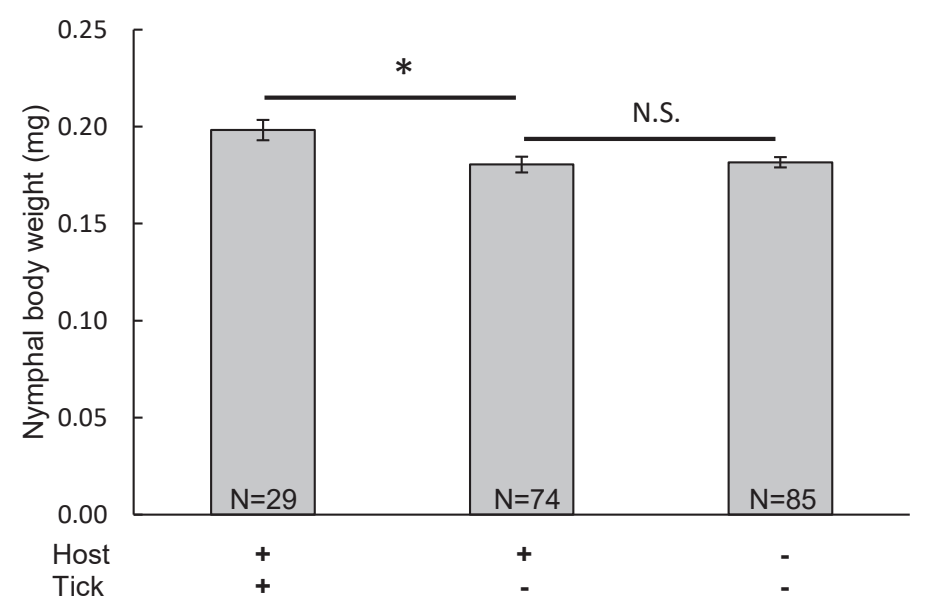

Figure 3. Average body weight ( \pm S.E.) of infected and uninfected nymphs that fed as larvae on infected or uninfected bank voles. $+=B$. afzelii-infected, $-=B$. afzelii-uninfected, $N=$ number of emerged nymphs. Significance indications are illustrated as * $\mathrm{P}<0.05$, N.S. $=$ not significant.

\section{Discussion}

We showed that $I$. ricinus nymphs can sense bank vole presence from air containing animal emanations. Nymphs preferred air containing bank vole emanations over ambient air. These emanations most likely consist of odours because each stream of air was led through a humidifier, aligning air humidity and temperature. This observation is in agreement with the preference of Dermacentor variabilis for air from white footed mice compared to clean air (Dallas and Fore 2013) and the response of $I$. scapularis adults and $I$. ricinus nymphs to host odours (Carroll et al. 1995, Crooks and Randolph 2006). In addition, ticks are also able to distinguish hosts species from their volatile emanations, as was shown for $I$. scapularis and $I$. pacificus larvae that preferred the odours from mice compared to that from chipmunks and chicken (James and Oliver Jr 1990, Shaw et al. 2003). The collective data suggests that members of the genus Ixodes respond to host odours, just like many other arthropods (Bursell 1984, Guerenstein and Guerin 2001, Carey et al. 2010), to correctly identify hosts (Takken and Verhulst 2013).

In addition, we showed that $I$. ricinus nymphs are more attracted to bank voles infected with $B$. afzelii compared to uninfected bank voles. There are several examples of endoparasite infections that can modify the odour profile of rodents making them more or less attractive to conspecifics (Kavaliers and Colwell 1995a, b, Penn et al. 1998, Klein et al. 1999, Willis and Poulin 2000, Barthélémy et al. 2005). Specific to vector-borne diseases it has recently been shown that a Plasmodium infection alters host odour, making mice more attractive to host seeking mosquitoes (De Moraes et al. 2014). 
However, another possible explanation for our finding is that the preference for bank voles infected with $B$. afzelii was based on a difference in $\mathrm{CO}_{2}$ production by the bank voles. Carbon dioxide is a waste product of animal metabolism. Rodents produce antibodies after a $B$. burgdorferi s.I. infection (Schwanz et al. 2011a), which may increase energy demand of the host (Murray et al. 1998). A higher $\mathrm{CO}_{2}$ production of the infected rodents may, therefore, have attracted more ticks than the uninfected rodents. Carbon dioxide is a commonly used host derivative inducing host-seeking behavior in hematophagous insects and ticks (Garcia 1962, Takken and Kline 1989, Koenraadt and Dicke 2010, Chaisson and Hallem 2012) and is often used in combination with host odour (Webster et al. 2015). Whether $\mathrm{CO}_{2}$ production of the bank voles was affected by a $B$. afzelii infection needs more research. A preference of ticks for bank voles infected with $B$. afzelii may lead to a higher larval tick burden on infected bank voles, and subsequently to a higher infection rate of nymphal populations resulting from the infected larvae. Under semi-field conditions we show that infected bank voles acquire a higher larval tick burden than uninfected voles. This difference was, however, only found in male and not in female bank voles. This effect of sex might be caused by a difference in the physiology between the sexes. More research is needed to investigate why the effect of a $B$. afzelii infection on attractiveness was dependent on host sex. A higher tick burden can result in a higher metabolic rate of the rodent, as was found for the number of $I$. persulcatus feeding on Myodes rutilus (Novikov et al. 2015). A higher metabolic rate leads to a higher energy demand and, therefore, a higher foraging activity, which may intensify the effect on tick burden. Our data supports that there is not one single factor explaining the heterogeneity of tick burden, but that this is caused by rather complex combinations of factors (Brunner and Ostfeld 2008, Van Duijvendijk et al. 2015).

In addition, we also demonstrated that, when fed on an experimentally infected bank vole, infected nymphs had a higher body weight than uninfected nymphs. Ixodes ricinus larvae need only one successful blood meal before they moult into nymphs. The size of this blood meal positively influenced nymphal body weight, energy storage of the emerged nymph and, therefore, nymphal survival (Bize et al. 2008, Gassner 2010, Herrmann and Gern 2010)Van Duijvendijk et al. submitted). A larger body weight of ticks infected with $B$. burgdorferi s.l. was also found in field-collected I. ricinus nymphs (Gassner 2010, Herrmann et al. 2013b) and $I$. scapularis infected with Babesia microti (Randolph 1991, Hu et al. 1997b). These positive effects of pathogen infections on blood meal size may be explained by a suppressive effect of the infection on the host's immune system, or an inhibited haemostasis, allowing for a larger blood meal size (Minchella 1985, Alugupalli et al. 2003, Rupprecht et al. 2008). However, we show that body weight of uninfected nymphs was not influenced by a $B$. afzelii infection in the host, excluding an immunosuppressive effect on the host's immune system and an inhibited haemostasis. Due to the positive relation between the body weight of engorged larvae and the 
body weight of emerged nymphs (Van Duijvendijk et al. submitted), we suggest a relation between a $B$. afzelii infection in the tick and larval blood meal size. A large blood meal may increase spirochete uptake (Raberg 2012), which may have positively affected infection probability of the ticks. However, if blood meal size would determine tick infection probability, mean body weight of nymphs from infected bank voles (group 1 and 2) and nymphs from uninfected bank voles (group 3) would be equal. Larvae feed for about four days, whereas $B$. burgdorferi s.l. can be transmitted from the host to feeding larvae after $8 \mathrm{~h}$ of feeding and $76 \%$ of the feeding larvae acquired an infection after $48 \mathrm{~h}$ of feeding (Piesman 1991). We, therefore, suggest that $B$. afzelii spirochetes stimulated or facilitated the larvae to consume more blood when attached to the midgut wall. In some cases, pathogens have been shown to reduce blood meal size of vectors to increase vector-host contact rate (Molyneux and Jefferies 1986). For the tick- $B$. afzelii system, however, a reduced blood meal size does not enhance pathogen transmission. An increased blood meal size, on the other hand, could be beneficial for tick survival if it results in a higher energy storage and, therefore, enhances transmission from nymph to rodent. How much this higher body weight contributes to the chance that a questing nymph acquires a blood meal and transmits the spirochetes to a rodent needs further research to determine the total effect of the $B$. afzelii infection on the $B$. afzelii lifecycle.

Our results show that an infection with Lyme borreliosis spirochetes affects emanations of its reservoir host and body weight of its vector. This results in increased contact rate between host and vector, enhancing the lifecycle of $B$. afzelii. It cannot be excluded that these effects are the result of a coincidentally beneficial general infection caused by the parasite, suppressing the hosts' and ticks' immune systems and not of truly adaptive active manipulation of the parasite (Thomas et al. 2005, Cator et al. 2013). However, irrespective of the specificity, the pathogen-induced changes enhance parts of the enzootic cycle of $B$. afzelii. The pathogenmediated intra-species host preference of $I$. ricinus provides novel ecological insights into the epidemiology of $B$. afzelii.

\section{Acknowledgements}

We thank Jeroen Coumou and Anneke Oei for supplying the $B$. afzelii spirochaetes and Wilma Blauw for her help with the inoculations of the bank voles. 



\section{Chapter 6}

\section{Rodents as hosts for Ixodes ricinus and Borrelia afzelii}

Gilian van Duijvendijk, Gerrit Gort, Willem Takken 


\section{Abstract}

Ixodes ricinus is the vector of the Lyme borreliosis-causing bacterium Borrelia afzelii in Europe. Larvae of $I$. ricinus mainly feed on rodents, which are reservoir hosts of $B$. afzelii. Rodent species vary in their suitability as hosts for ticks and $B$. afzelii. The effects of rodent species on tick burden, host infection rate and infectivity are reviewed and the effect of rodent species on tick physiology was studied in a field experiment. Wood mice (Apodemus sylvaticus) and bank voles (Myodes glareolus) were trapped in the field and body weight of engorged larvae and flat nymphs was measured. Body weight of engorged larvae and flat nymphs was higher for ticks that fed on wood mice compared to ticks that fed on bank voles. Overall, we conclude that wood mice are better hosts for ticks but that bank voles are better hosts for $B$. afzelii. The density of a rodent species, which is largely affected by food availability, has, however, the largest effect on determining the contribution of a rodent species to the density of infected nymphs and, therefore, Lyme borreliosis risk. 


\section{Introduction}

The sheep tick (Ixodes ricinus, Acari: Ixodidae) is the principal vector of the Lyme borreliosiscausing spirochaete Borrelia burgdorferi sensu lato (s.l.) in Europe. Borrelia burgdorferi s.I. is transmitted to humans through the bite of an infected tick, usually a nymph (Hofhuis et al. 2013). The density of infected ticks, therefore, positively affects Lyme borreliosis incidence (Stafford III et al. 1998, Diuk-Wasser et al. 2012). The density of infected nymphs is calculated by multiplying the density of nymphs with the proportion of infected nymphs (hereafter referred to as the infection rate). So, when one of these parameters increases, the chance of getting bitten by an infected nymph increases. Rodents are commonly used as blood hosts by tick larvae and are the natural reservoir host for $B$. afzelii, one of the most common $B$. burgdorferi s.l. genospecies in Europe (Hanincová et al. 2003a, Rauter and Hartung 2005). Rodent reservoir hosts, therefore, contribute strongly to both the density of nymphs and the infection rate of nymphs (Tälleklint and Jaenson 1994, Tälleklint and Jaenson 1995). Most rodents have zero to a few ticks and only a small proportion of the rodent populations feeds the majority of ticks (Randolph et al. 1999, Perkins et al. 2003). An individual rodent can feed over 300 ticks, depending on the geographical location, season and year. The role of rodents as hosts for ticks and $B$. afzelii and the differences between two common rodent species in The Netherlands are discussed in this chapter.

\section{Tick ecology}

Ixodes ricinus has four developmental stages: egg, larva, nymph and adult. Each of the three motile stages must take a blood meal to complete their development to the next stage. All tick stages climb up the vegetation and wait for a vertebrate host, a behaviour called questing. During questing, evaporative water loss forces questing ticks to return to the litter layer to restore their moisture content (Lees 1946, Perret et al. 2003). Questing duration and questing height are, therefore, influenced by the microclimate around the tick (Randolph and Storey 1999). Questing immature ticks (larvae and nymphs) are activated by $\mathrm{CO}_{2}$ from the host and are attracted to host odour (Berret and Voordouw 2015; Van Duijvendijk et al. submitted). After encountering a host, the tick stretches its forelegs, holds on to the passing host and searches for a suitable place on the host to bite and feed for about 3 to 7 days (depending on the stage). When the tick is fully engorged, it drops from the host and searches for a place in the moist litter layer to digest its blood meal and moult into the next stage. Ixodes ricinus feeds on a wide variety of vertebrate hosts including: rodents, deer, birds and reptiles (Keirans et al. 1996, Hofmeester et al. 2016). The height at which the ticks quest affects the size of the encountered hosts and differs between the three tick stages. Larvae quest at heights of $0-10 \mathrm{~cm}$, nymphs 
at $30-60 \mathrm{~cm}$ and adult ticks mainly quest at $60-80 \mathrm{~cm}$ above the ground (Mejlon and Jaenson 1997). As a result, questing larvae mainly encounter small mammals like rodents, which are important blood hosts for larvae (Matuschka et al. 1991).

\section{Rodent ecology}

In Europe, bank voles (Myodes glareolus, Rodentia: Cricetidae), wood mice (Apodemus sylvaticus, Rodentia: Muridae) and yellow-necked mice (Apodemus flavicollis, Rodentia: Muridae) (Figure 1) are the most common rodents in tick habitat and are, therefore, often used as blood hosts by larvae. These rodent species can occur together in the same region due to the different niches they occupy (Khanakah et al. 2006). In The Netherlands, the distribution of the yellow-necked mouse is limited, whereas wood mice and bank voles are widespread throughout the country. The abundance of these rodent species is regulated by different factors, like food availability, predation and vegetation cover (Zwolak et al. 2016) and, therefore, varies temporally and geographically. Food availability is one of the main environmental factors that has a large effect on rodent abundance (Ostfeld et al. 2006, Bogdziewicz et al. 2016). The rodents under study are herbivores feeding on the seeds of a variety of plant species. Large tree species, like oak and beech do not produce seeds every year, but produce their seeds synchronously depending on the weather conditions, called masting (Kelly and Sork 2002). The phenomenon causes fluctuations in rodent densities between years and geographic locations, depending on the presence of mast the species and climatic conditions (Ostfeld et al. 1996, Ostfeld and Keesing 2000). Because rodents are the most important hosts for tick larvae, masting increases the density of ticks (Jones et al. 1998).

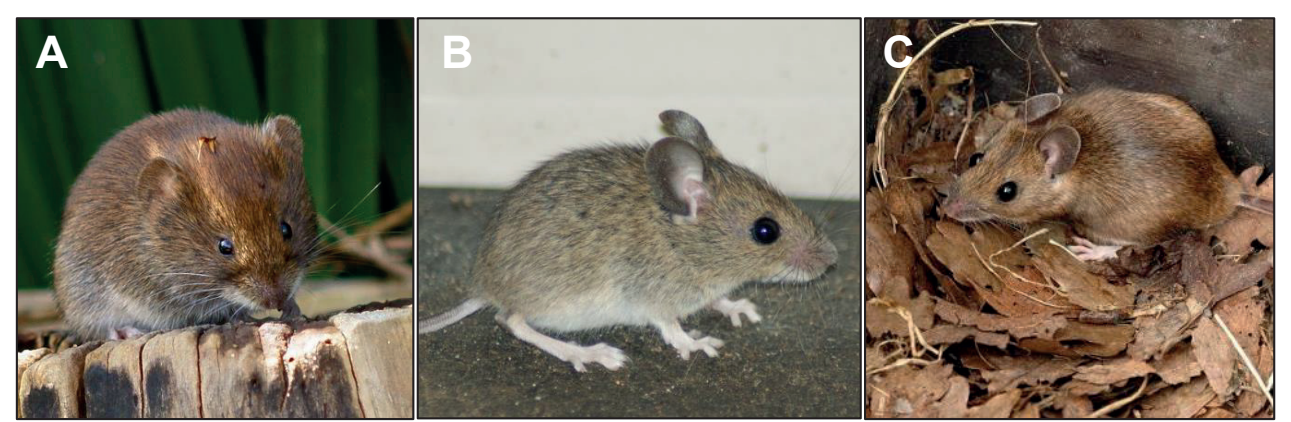

Figure 1. The three most common rodent species used by larval Ixodes ricinus. Bank vole (A), wood mouse (B) and yellow necked mouse (C). Copy right: bank vole: Peter Trimming, CC BY 2.0, wood mouse: Spencer Wright, CC BY 2.0, yellow-necked mouse: James Lindsey, CC BY-SA 3.0. 


\section{Rodents as hosts for $B$. afzelii}

Lyme borreliosis is caused by spirochaetes from the Borrelia burgdorferi s.l. complex. This complex consists of 19 genospecies, which can cause different clinical symptoms in humans (Nau et al. 2009). The different genospecies are all transmitted by I. ricinus, but their enzootic lifecycles depend on different natural reservoir hosts. In Europe, the most common genospecies is B. afzelii (Rauter and Hartung 2005, Herrmann et al. 2013a), which can cause skin manifestations (Stanek and Strle 2003, Strle and Stanek 2009). Borrelia afzelii circulates between ticks and rodents, whereas $B$. garinii circulates between ticks and birds (Kurtenbach et al. 1998b, Humair et al. 1999, Hanincová et al. 2003a, Hanincová et al. 2003b, Kybicova et al. 2008). Rodents are born free of Lyme borreliosis spirochaetes (Mather et al. 1991) and can become infected with $B$. afzelii via the bite of an infected larva or nymph (Radolf et al. 2012, Van Duijvendijk et al. 2016), after which they will remain infected throughout their life (Gern et al. 1994). In contrast to Ixodes trianguliceps, adult $I$. ricinus ticks rarely feed on rodents and are, therefore, not considered to contribute to the lifecycle of $B$. afzelii. Therefore, $B$. afzelii cycles from infected questing larvae or nymphs, to rodents and from rodents to feeding larvae, which subsequently develop into infected questing nymphs (Figure 2). This cycle is influenced by different characteristics of the rodent host, tick vector and Borrelia pathogen (Van Duijvendijk et al. 2015).

In general, bank voles are better hosts for $B$. afzelii than wood mice because they have a higher host infection rate (Tälleklint and Jaenson 1994, Humair et al. 1999, Kybicova et al. 2008, Gassner et al. 2013) and the probability that a feeding larvae acquires $B$. afzelii from a rodent (infectivity), is also higher for bank voles (Kurtenbach et al. 1995, Humair et al. 1999, Perez et al. 2012, Radzijevskaja et al. 2013). A higher B. afzelii spirochaete load in rodent tissue has a positive effect on its infectivity to feeding I. ricinus larvae (Raberg 2012), but the effect may be counteracted by the blood meal size of the tick. The contribution of a rodent to the density of infected nymphs is not only affected by their suitability as host for $B$. afzelii, but is also affected by their suitability as host for $I$. ricinus. The suitability of rodents as host for $I$. ricinus is discussed in the next paragraph. 


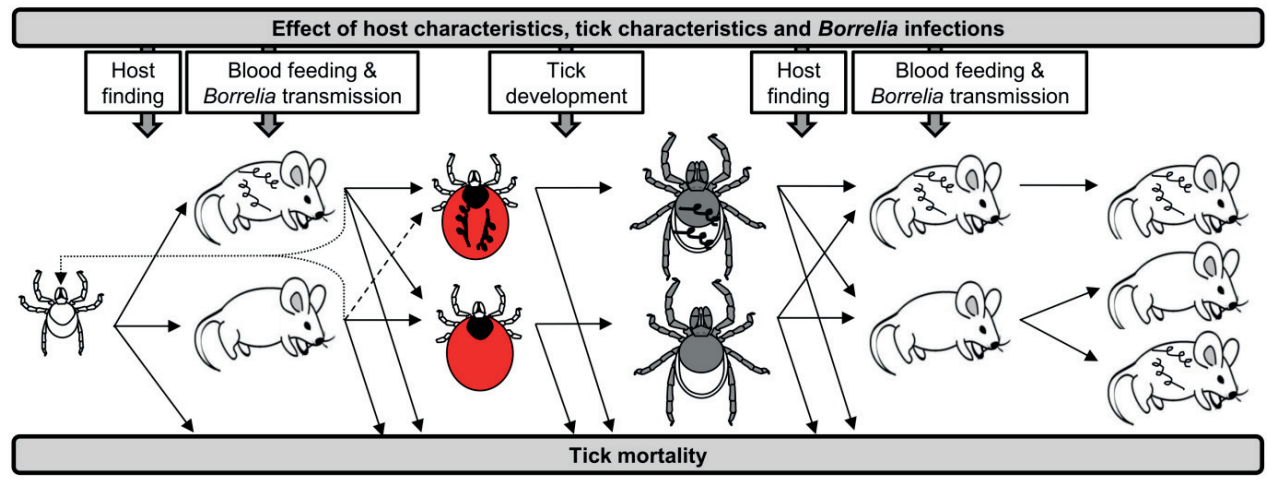

Figure 2. Schematic overview of the development from an uninfected Ixodes ricinus larva to a nymph infected with $B$. afzelii and the transmission process of $B$. afzelii between rodent and tick. Dotted lines indicates continuation of questing after a partial blood meal, dashed line indicates co-feeding transmission. Taken from Van Duijvendijk et al. (2015).

\section{Rodents as blood hosts for Ixodes ricinus}

The contribution of rodents to the density of infected nymphs depends on their tick burden, their $B$. afzelii infection status, the probability that feeding larvae will acquire spirochetes, the probability that infected engorged larvae moult into infected nymphs and the longevity of the infected nymphs. When rodent density is increased, for example in the year after a mast year (Clotfelter et al. 2007), the tick larvae that quest for a host have a higher chance of encountering a rodent. As a result, nymphal density is expected to increase the following year (Ostfeld et al. 2006). The average number of ticks feeding per rodent declines with high rodent densities (Schmidt et al. 1999, Brunner and Ostfeld 2008), but the total number of feeding larvae per unit area was still higher due to the high rodent density (Rosa et al. 2007), explaining the positive effect of rodent density the density of nymphs the following year.

Rodent species differ in their suitability as hosts for ticks. Several studies have analysed the effects of rodent species on tick burden. In general, mice have a greater larval tick burden than bank voles (Nilsson and Lundqvist 1978, Humair et al. 1993, Kurtenbach et al. 1995, Tälleklint and Jaenson 1997, Gray et al. 1999, Boyard et al. 2008, Kiffner et al. 2011, Gassner et al. 2013), which is expected to be the result of an acquired immunity of bank voles to feeding ticks (Dizij and Kurtenbach 1995). It is difficult to determine the contribution of rodents to feeding immature ticks compared to other vertebrate hosts. Doing so requires knowledge about the abundance and mean tick burden for all of the vertebrate host species in the community. Tick burden data was estimated for a vertebrate community in Europe (Tälleklint and Jaenson 1997, Hofmeester et al. 2016) and the US (LoGiudice et al. 2003, Brisson et al. 2008, Keesing et al. 2009). One innovative (but difficult) approach is to use host blood meal analysis to estimate 
the contribution of the different vertebrate hosts to feeding immature ticks (Pichon et al. 2003, Pichon et al. 2005, Morán-Cadenas et al. 2007, Scott et al. 2012). In Switzerland, host blood meal analysis showed that rodents feed about $20 \%$ of the larval ticks (Morán-Cadenas et al. 2007).

The higher tick burden on mice compared to bank voles can be the result of either a preference of the tick for one host species over another, or phenotypic differences (e.g. microhabitat preferences, grooming behaviour, immunological differences) between rodent species. Not much is known about the host preference and selection of I. ricinus (McCoy et al. 2013), whereas host preference under laboratory conditions was shown for $I$. scapularis and $I$. pacificus (Shaw et al. 2003, Slowik and Lane 2009). Wood mice and bank voles use different ecological niches. Van Duijvendijk et al. (accepted) showed that a $B$. afzelii infection in rodents can also affect tick burden; it changed the odour of the rodents and made them more attractive to ticks. In addition, these authors also found that $B$. afzelii infection affects tick physiology; infected nymphs were heavier than uninfected nymphs. The effect of rodent species on the physiology of feeding $I$. ricinus larvae is largely unknown and was determined in an experiment described below.

\section{Experiment: Effect of rodent species on tick physiology.}

To determine the effect of a rodent species on the physiology of $I$. ricinus, we trapped rodents with their naturally attached ticks in their natural environment. Rodents were trapped in a forest near Wageningen, The Netherlands, using 144 live traps baited with grain, carrots and hay. Traps were set in the afternoon and inspected the following morning from May to November at three-week intervals (10 trapping occasions). Trapped female rodents were released at the trapping site. Trapped male bank voles and male wood mice were taken to the laboratory and housed individually in cages over pans with water. The attached larvae were allowed to complete their blood meal and the engorged larvae were collected after they dropped off their host and into the water. Engorged larvae were dried for $2 \mathrm{~h}$ on filter paper, weighed to the nearest microgram and housed individually at $20^{\circ} \mathrm{C}$ and $90 \% \mathrm{RH}$ to digest their blood meal and moult into flat nymphs. The engorged larvae were checked weekly, flat nymphs were reweighed and stored at $-20^{\circ} \mathrm{C}$.

The effect of rodent species on the body weight of engorged larvae and flat nymphs was analysed using mixed linear models with random effects for individual mice. Larval tick burden and rodent body weight had no effect on the body weight of engorged larvae and flat nymphs and were excluded from the models. The log of the body weight of engorged larvae was related to the log of the body weight of emerged nymphs, testing for differences between rodent species, using a mixed linear model with engorged larval weight as covariate, rodent species 
as factor and their interaction and random effects for mouse. All analyses were performed with SAS statistical software (SAS Institute Inc.), version 9.3.

Forty-three male bank voles and 28 male wood mice were trapped and yielded 509 and 651 engorged larvae, respectively. The mean tick burden of the wood mice $(23.3 \pm 4.7)$ was twofold higher than that of the bank voles $(11.8 \pm 1.9)$. The engorged larvae that had fed on wood mice had a higher body weight $(0.541 \pm 0.009 \mathrm{mg})$ than the larvae that had fed on bank voles $(0.447 \pm 0.008 \mathrm{mg}, P<0.0001$, Figure $3 \mathrm{~A})$. The nymphs that emerged from the engorged larvae that had fed on wood mice had a higher body weight $(0.247 \pm 0.005 \mathrm{mg})$ compared to the nymphs emerged from the larvae that had fed on bank voles $(0.181 \pm 0.004 \mathrm{mg}, \mathrm{P}<0.0001$, Figure $3 \mathrm{~B}$ ). In addition, the log body weight of the emerged nymphs was positively related to the log body weight of the engorged larvae for ticks that fed on wood mice $(P<0.0001)$ and on bank voles $(P<0.0001$, Figure 4). Interestingly, the regression coefficient was higher $(P<0.001)$ for ticks that fed on wood mice (Log body weight emerged nymphs $=-0.3195+1.1054{ }^{*} \log$ body weight of engorged larvae) than for ticks that fed on bank voles (Log body weight emerged nymphs $=-0.4171+0.9594$ * $\log$ body weight of engorged larvae). This observation may suggest that the ability of the larval tick to convert rodent blood into nymphal weight was more efficient for wood mice than bank voles. Because total body weight of the flat nymphs was measured, it is, however, unknown whether the steeper slope for wood mouse blood was caused by 1) a higher tissue weight, 2) fat storage, or 3) undigested blood in the flat nymphs. Previous work has shown that the body weight of nymphs was positively related to fat reserves and nymphal survival (Crooks and Randolph 2006, Herrmann and Gern 2010, Herrmann et al. 2013b). Further experiments are needed to clarify the relationship between nymphal body weight and nymphal survival under natural conditions and, therefore, the contribution of each rodent species to the density of infected nymphs. 

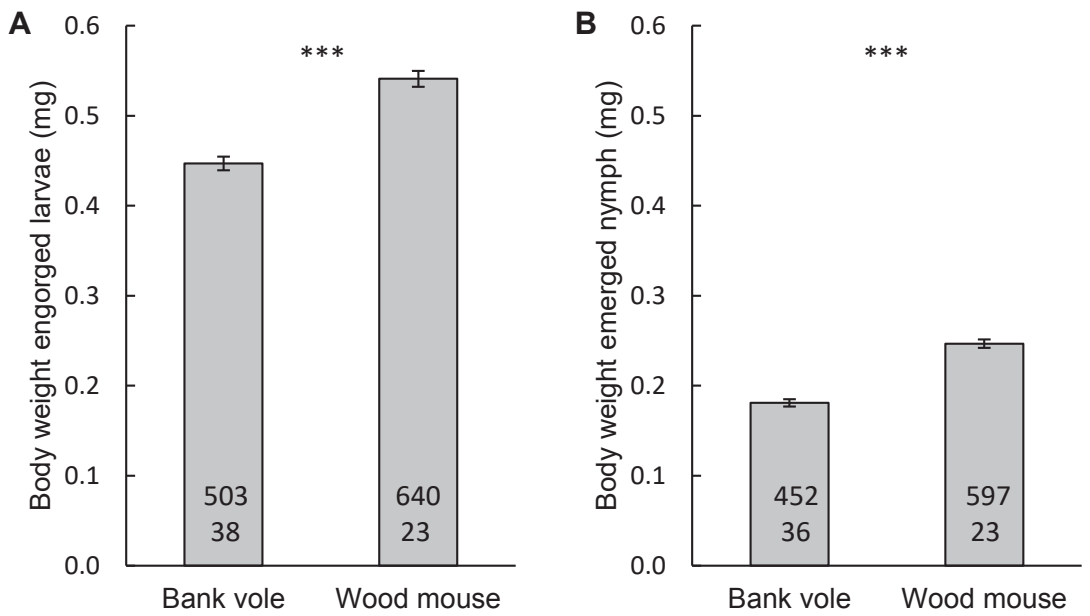

Figure 3. Body weight of engorged larvae (A) and body weight of emerged nymphs (B) of Ixodes ricinus that had naturally attached to bank voles or wood mice. Top numbers inside bars represent number of ticks, bottom numbers represent number of rodents, error bars represent standard errors. Significance is illustrated as ${ }^{* * *}=P<0.0001$.

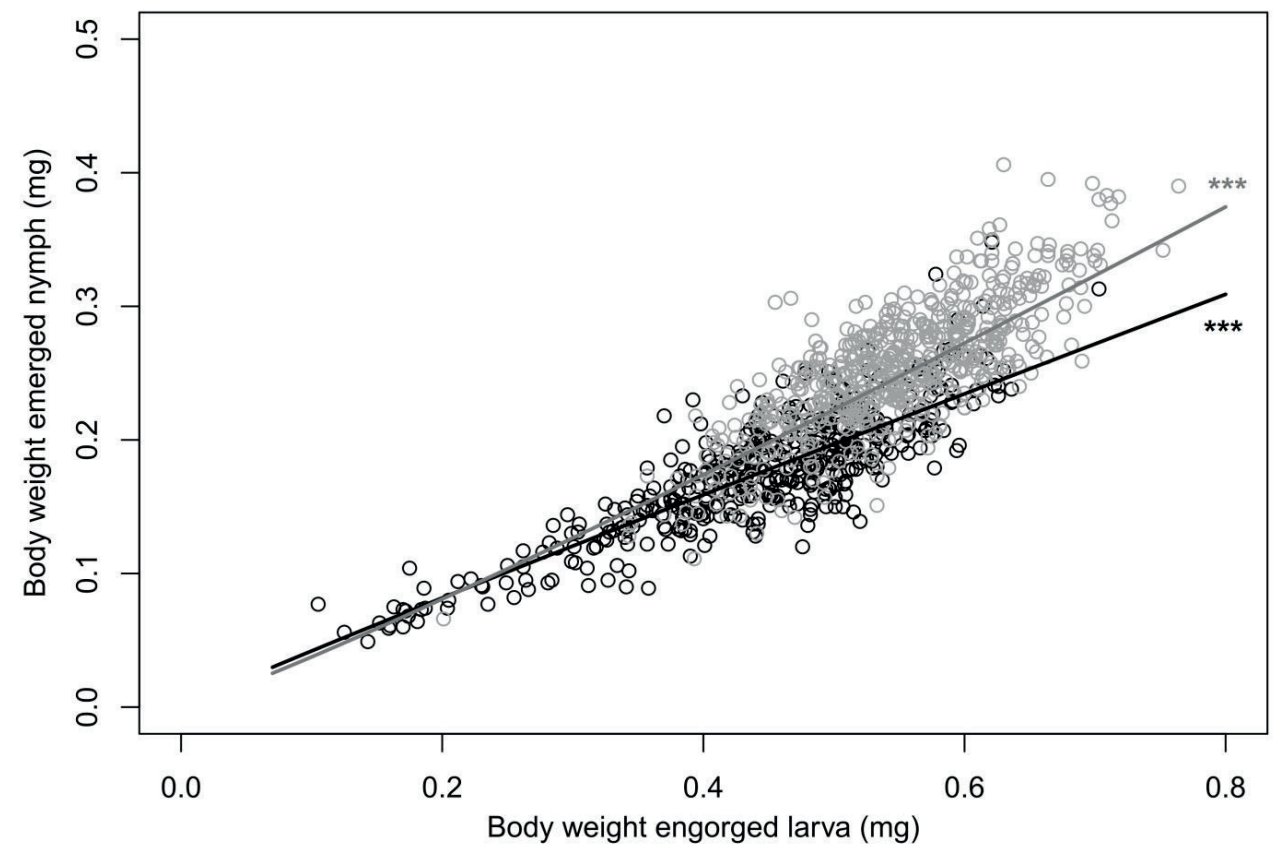

Figure 4. Relationship between body weight of engorged larvae and body weight of emerged nymphs for ticks that fed on field-collected bank voles (black circles) or wood mice (grey circles) collected from May 2013 till November 2013. Each dot represents one tick. Lines represent back transformed linear regression lines for bank voles (black) and wood mice (gray). Significance is illustrated as *** $=P<0.0001$. 


\section{Discussion}

We have shown that rodents are important in maintaining the lifecycles of $I$. ricinus and $B$. afzelii. They are important hosts for larvae and a high density of rodents, therefore, increases the probability that questing larvae find a host, resulting in an increase in the density of nymphs in the following year. The most common rodent species in The Netherlands differ in their suitability as hosts for $I$. ricinus. Bank voles, but not mice, have an innate resistance against feeding ticks and can develop acquired resistance against ticks over successive infestations, resulting in a lower tick attachment success, lower tick feeding success and lower larva-tonymph moulting success (Dizij and Kurtenbach 1995, Hughes and Randolph 2001). This would increase the relative contribution of wood mice to the density of nymphs compared to the contribution of bank voles. We found that this resistance also affects tick physiology under field conditions. Larvae were able obtain a larger blood meal from wood mice, compared to bank voles, resulting in heavier nymphs. The proteins in the larger blood meal during the larval stage are stored as fat in the nymphal body, which is expected to enhance nymph survival (Randolph and Storey 1999, Herrmann and Gern 2015), increasing the density of nymphs. In addition, we also found that the ability of larval ticks to convert rodent blood to nymphal body mass was more efficient for wood mice than bank voles. An effect of host species on tick physiology was also found for $I$. scapularis (Brunner et al. 2011, Jones et al. 2015). The authors, are, however, unaware of studies that show the effect host species on blood conversion. This topic requires further research to determine what caused this effect.

Considering Lyme borreliosis risk, infection prevalence in ticks is an important factor affecting the density of infected nymphs. Rodent infection rate and rodent infectivity also differ between the most common rodent species in The Netherlands. These two factors affect nymphal infection prevalence and are lower in wood mice compared to bank voles (Tälleklint and Jaenson 1994, Kurtenbach et al. 1995, Humair et al. 1999, Kybicova et al. 2008, Perez et al. 2012, Gassner et al. 2013, Radzijevskaja et al. 2013). Infectivity may, however, not always differ between mice and voles (Raberg 2012). At equal rodent densities, wood mice appear to contribute more to the density of nymphs than bank voles due to a higher larval tick burden on wood mice (Humair et al. 1993, Kurtenbach et al. 1995) and survival of the emerged nymphs from wood mice. This is in line with the findings of Perez et al. (2016), who concluded that wood mice density in spring positively affected the density of nymphs one year later, whereas this was not found for bank vole density. These authors contributed the higher contribution of wood mice to the higher larval tick burdens on wood mice compared to bank voles. The densities of wood mice and bank voles depend on different ecological factors, and the (relative) density of a given rodent species obviously determines the contribution of that rodent species to the total number of feeding larvae per unit area, and hence, on the density of nymphs the 
following year (Brunner and Ostfeld 2008, Perez et al. 2016). The contribution of these rodent species to the density of infected nymphs, may also interact with each other. When the density of wood mice and bank voles are not equal, the effects may change. A relatively low density of wood mice may increase tick burden, and therefore, acquired resistance and infection rate in bank voles. A Low density of bank voles, may, however result in a lower nymphal infection prevalence and, consequently infection rate in wood mice one year later. Rodents are important for I. ricinus larvae and B. afzelii (Kurtenbach et al. 2002a, Hanincová et al. 2003a, Hofmeester et al. 2016) and rodent density does, therefore affect the density of infected nymphs one year later (Ostfeld et al. 2001, Ostfeld et al. 2006). Rodents are a challenging target for ecological interventions that aim to reduce Lyme borreliosis incidence. Rodent density varies geographically and annually and due to the high numbers of rodents, intervening in their population size, without affecting overall biodiversity is difficult. Rodent density may be used as a predictor for the density of infected nymphs in the next year.

\section{Conclusions and public health relevance}

- Rodents are important blood hosts for I. ricinus larvae.

- Rodents are the reservoir host for $B$. afzelii and can transmit the spirochaetes to the larvae that feed on them. Infected larvae moult into infected nymphs, which are responsible for transmitting the spirochaetes to humans.

- There are large differences between the contributions of the most common rodent species to Lyme borreliosis risk in terms of tick burden, rodent infection rate, infectivity and tick physiology.

- Rodents may be a good predictor for the density of nymphs, but their use in intervention studies may be challenging due to high spatiotemporal variation in their density.

\section{Acknowledgements}

We thank Marloes van Schaijk for her help with the experiment and Hein Sprong and Sip van Wieren for their comments on an earlier version of this chapter. 



\section{Chapter 7}

\section{Behavioural responses of Ixodes \\ ricinus nymphs to carbon dioxide and rodent odour}

Gilian van Duijvendijk, Gerrit Gort ,Hein Sprong, Willem Takken 


\section{Abstract}

Many haematophagous ectoparasites use $\mathrm{CO}_{2}$ and host odour to detect and locate their host. The tick Ixodes ricinus (Linnaeus, Acari: Ixodidae) only walks small distances and quests in the vegetation until a host is encountered. The differential effect of $\mathrm{CO}_{2}$ and host odour on the host finding behaviour of $I$. ricinus has, however, never been clarified and is the subject of this study.

The effect of $\mathrm{CO}_{2}$ and odour from bank voles on the activation and attraction of $I$. ricinus nymphs was analysed in a Y-tube olfactometer. Carbon dioxide evoked a response in absence and presence of host odour, but did not attract nymphs. Host odour, however, did not evoke a response but attracted nymphs in absence and presence of $\mathrm{CO}_{2}$.

Our results show that $\mathrm{CO}_{2}$ is an activator but not an attractant and that host odour is an attractant, but not an activator of $I$. ricinus nymphs, providing ecological insights into the hostfinding behaviour of $I$. ricinus. 


\section{Introduction}

The sheep tick (Ixodes ricinus) is a vector of a variety of medically and veterinary important pathogens, including Borrelia burgdorferi sensu lato, Borrelia miyamotoi, tick-borne encephalitis virus, Anaplasma phagocytophilum, Candidatus Neoehrlichia mikurensis, Rickettsia helvetica, Rickettsia monacensis, Babesia microti, B. divergens, and Louping ill virus (Parola and Raoult 2001, Dantas-Torres et al. 2012). Larvae, nymphs and adults of $I$. ricinus quest in the vegetation for a host to acquire a blood meal for their interstadial development or the production of eggs in the case of adult females. Questing activity is dependent on environmental factors like temperature and relative humidity (Daniel et al. 2015). During questing, host emanations (kairomones) are detected by the tick for host recognition and after encountering a host, the tick attaches to it and searches for a suitable feeding place. Carbon dioxide and host odour are commonly used kairomones in haematophagous arthropods, including ticks, and induce host-seeking behaviour (Garcia 1962, Carroll et al. 1995, Chaisson and Hallem 2012). The differential effect of $\mathrm{CO}_{2}$ and host odour on the host-seeking behaviour of $I$. ricinus has, however, never been clarified. The aim of this study is, therefore, to investigate the differential effect of $\mathrm{CO}_{2}$ and host odour on the host-seeking behaviour of $I$. ricinus.

\section{Materials and Methods}

\section{Study organisms}

The study was done with Ixodes ricinus nymphs. Nymphs were collected by dragging a 1*1m white cotton blanket over low vegetation and leaf litter in a forest $\left(52^{\circ} 01^{\prime} 36^{\prime \prime} \mathrm{N}, 5^{\circ} 41^{\prime} 21^{\prime \prime} \mathrm{E}\right)$ near Wageningen, The Netherlands in August/September 2014. Ticks that attached to the blanket were collected and housed in $50 \mathrm{ml}$ tubes at room temperature, $90 \% \mathrm{RH}$ and a natural photoperiod and were used within one month after capture. Bank voles (Myodes glareolus) are commonly used as blood host by $I$. ricinus. Therefore, 16 captive-bred bank voles of two to four months old were used as sources of host odour. Bank voles were handled with gloves to prevent contact with human odour. Male and female bank voles were used and each bank vole was used only once in each treatment (experiment 2). Prior to the study, all experiments had been approved by the ethical committee of Wageningen University (no. 2012065). 


\section{Bioassays}

A closed-system Y-tube olfactometer (Takabayashi and Dicke 1992) was used to test the effects of $\mathrm{CO}_{2}$ and host odour on tick behaviour ( $25 \pm 2{ }^{\circ} \mathrm{C}$ and $740 \mathrm{Lux}$ ). Two glass $\mathrm{Y}$-tube olfactometers (arms: $7 \mathrm{~cm}$, stem: $8 \mathrm{~cm}$, diameter: $8 \mathrm{~mm}$ ) were positioned at an upward angle of $45^{\circ}$. Streams of pressurized air (1.2 l/min, Sho-rate rotameter, Brooks Instrument B.V.) were humidified to $>90 \%$ by leading the flows through $1 \mathrm{I}$ bottles filled with tap water and led to the arms of the olfactometer.

In experiment 1 , the effect of $\mathrm{CO}_{2}$ in the absence of host odour on nymph activation and attraction was tested. Different flow rates of $100 \% \mathrm{CO}_{2}$ were added to the air streams to maintain an ambient $\mathrm{CO}_{2}$ concentration or create $\mathrm{CO}_{2}$ concentrations of 0.4 or $4 \%$ above the ambient concentration. Six treatments were tested (Figure 1).

In experiment 2, the effects of $\mathrm{CO}_{2}$ and host odour on nymph activation and attraction were tested. Now each stream was first led through a 5 I glass animal holding chamber, which contained either no bank vole or one chamber contained one bank vole while the other was left empty. Bank voles were placed in the holding chamber $10 \mathrm{~min}$. before the start of the experiment. The setups were tested with and without a soda lime filter between the animal holding chamber and $\mathrm{Y}$-tube olfactometer to remove $\mathrm{CO}_{2}$ from the airflow. Four treatments were tested (Figure 2).

Per treatment, 32 groups of 10 nymphs were released from a small release cup that was connected to the stem of Y-tube olfactometer and their positions ("left", "right", or "no choice" when in the stem of the Y-tube) were recorded after $15 \mathrm{~min}$. Each nymph was used only once. Ticks were acclimatised to the conditions of the test room for at least $1 \mathrm{~h}$ prior to use. Odour sources were switched between the Y-tube arms and all materials were cleaned with $70 \%$ alcohol between replicates.

\section{Data analysis}

The effects of $\mathrm{CO}_{2}$ and host odour on nymph activation (number of nymphs in both arms per treatment, given the number of released nymphs) was analysed using a GLM, assuming a binomial distribution with logit link function, and using an extra scale parameter based on Pearson's chi-square to correct for overdispersion. Test date was included in the model as a block factor. Differences between treatments were tested by pair wise comparisons per experiment. The effects of $\mathrm{CO}_{2}$ and host odour on nymph attraction (number of nymphs in one arm given the total number of responding nymphs in both arms of the Y-tube olfactometer) was analysed per treatment using a GLM, assuming a binomial distribution with logit link function. Effects were considered significant at $P<0.05$. All analyses were performed with $S A S$ statistical software (SAS Institute Inc.), version 9.3. 


\section{Results}

\section{Effect of $\mathrm{CO}_{2}$ on tick activation and attraction}

In experiment 1 , nymphal response was higher in the situations where $4 \% \mathrm{CO}_{2}$ was added (treatment 4, 5, and 6) compared to the situation with ambient air (treatment 1) and the situation where $0.4 \% \mathrm{CO}_{2}$ was added to one arm of the $\mathrm{Y}$-tube (treatment $2, \mathrm{P}<0.05$, Figure 1). Nymphal response in the situation where $0.4 \% \mathrm{CO}_{2}$ was added to both arms of the $\mathrm{Y}$-tube (treatment 3 ) did not differ from the other treatments $(P>0.05)$. Nymphs did not show a preference for ambient air, ambient air $+0.4 \% \mathrm{CO}_{2}$ or ambient air $+4 \% \mathrm{CO}_{2}$ in any of the treatments $(P>0.05)$.

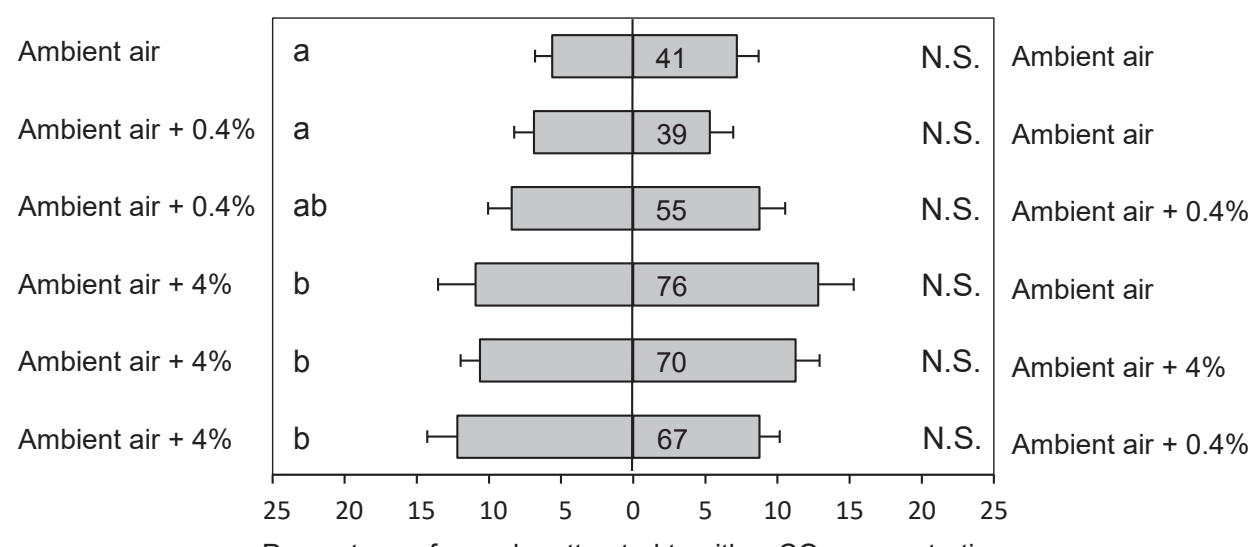

Percentage of nymphs attracted to either $\mathrm{CO}_{2}$ concentration

Figure 1. Experiment 1: Effect of $\mathrm{CO}_{2}$ on tick activation and attraction in a y-tube dual-choice assay. Numbers inside bars represent the total number of nymphs that responded out of 320 nymphs. Ambient $\mathrm{CO}_{2}$ concentration $\approx$ $0.03 \%$. Error bars represent S.E. of percentages of ticks that chose for either $\mathrm{CO}_{2}$ concentration, based on 32 replicates of 10 nymphs. Lack of a common letter to the left of the bars represent a significant difference in response between treatments (length of the bar, $\mathrm{P}<0.05$ ). Significance indications to the right of the bars represent choice differences within treatments (position of the bar), illustrated as N.S. = not significant.

\section{Effect of $\mathrm{CO}_{2}$ and host odour on tick activation and attraction}

In experiment 2, nymphal response was not affected by the presence of ambient $\mathrm{CO}_{2}$ when host odour was absent (treatment 1 vs treatment 2, $\mathrm{P}=0.321$, Figure 2). Nymphal response was higher when ambient $\mathrm{CO}_{2}$ or host-produced $\mathrm{CO}_{2}$ was present (treatment $3,17.5 \pm 1.8 \%$ ) compared to the absence of $\mathrm{CO}_{2}$ (treatment 4, $5.6 \pm 1.6 \%$ ) when host odour was present $(\mathrm{P}<0.001)$. Nymphal response was not affected by host odour in the absence of $\mathrm{CO}_{2}$ (treatment 2 vs treatment $4, \mathrm{P}=0.525$ ). Nymphal response was higher in the presence of host odour and 
host-produced $\mathrm{CO}_{2}$ (treatment $3,17.5 \pm 1.8 \%$ ) compared to the absence of host odour and presence of ambient $\mathrm{CO}_{2}$ (treatment $1,9.1 \pm 1.5 \%, \mathrm{P}=0.003$ ).

Nymphs did not show a preference for either stream of ambient air in the absence of ambient $\mathrm{CO}_{2}$ (treatment 2, $\mathrm{P}=0.396$ ). Nymphs preferred the airflow containing host odour in the absence of host-produced $\mathrm{CO}_{2}$ (treatment $4, \mathrm{P}=0.011$ ). Data on the lack of preference for either stream of ambient air in presence of ambient $\mathrm{CO}_{2}$ (treatment $1, \mathrm{P}=0.853$ ) and on the preference for host odour in presence of host-produced $\mathrm{CO}_{2}$ (treatment $3, \mathrm{P}<0.001$ ) was taken from Van Duijvendijk et al. (accepted) and reproduced here for direct comparison.

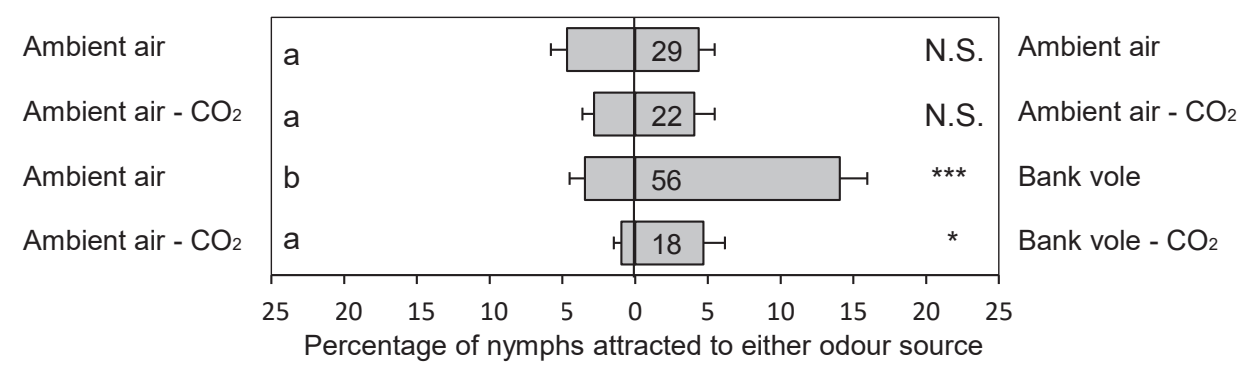

Figure 2. Experiment 2: Effect of $\mathrm{CO}_{2}$ and host odour on tick activation and attraction in a y-tube dual-choice assay. Numbers inside bars represent the total number of nymphs that responded out of 320 nymphs. Ambient $\mathrm{CO}_{2}$ concentration $\approx 0.03 \%$. In the second and fourth bar, $\mathrm{CO}_{2}$ was removed from the airflows using two soda lime filters. Error bars represent S.E. of percentages of ticks that chose for either odour source, based on 32 replicates of 10 nymphs. Lack of a common letter to the left of the bars represent a significant difference in response between treatments (length of the bar, $\mathrm{P}<0.01$ ). Significance indications to the right of the bars represent choice differences within treatments (position of the bar), illustrated as N.S. = not significant, ${ }^{* * *}=P<0.001,{ }^{*}=P<0.05$.

\section{Discussion}

In experiment 1 , we showed that $I$. ricinus nymphs respond to elevated $\mathrm{CO}_{2}$ concentrations in the surrounding air. This is in line with the results of Gherman et al. (2012), who showed that adding $\mathrm{CO}_{2}$ to a blanket to a concentration of $0.08 \%$ increased capture efficiency of $I$. ricinus and Gray (1985), who showed that a trap baited with $\mathrm{CO}_{2}$ caught I. ricinus larvae, nymphs and adults. An increased capture efficiency of $I$. ricinus is not necessarily the result of the attraction of ticks to the trap, but can also be the result of an increased activity of ticks induced by the $\mathrm{CO}_{2}$. Our results show that $I$. ricinus nymphs are activated by, but not attracted to $\mathrm{CO}_{2}$. This $\mathrm{CO}_{2}$-induced increase of activation is common in haematophagous arthropods, but had not been proven yet for $I$. ricinus. The increased activity of $I$. ricinus nymphs was found in a situation with an artificial $\mathrm{CO}_{2}$ concentration of $4 \%$ and in a situation with host-produced $\mathrm{CO}_{2}$ in combination with host odour. The $4 \% \mathrm{CO}_{2}$ concentration had been chosen to approximate the $\mathrm{CO}_{2}$ concentration exhaled by mammalian hosts. We, therefore, suggest that the host- 
seeking behaviour of $I$. ricinus nymphs (activation) is triggered by $\mathrm{CO}_{2}$. It is plausible that $\mathrm{CO}_{2}$, which is a waste product of the metabolism of all vertebrate host species, is used during the host-seeking behaviour of a generalist tick species like I. ricinus (Sonenshine 2004). In experiment 2, we found that rodent odour did not activate but attracted $I$. ricinus nymphs. The attraction of $I$. ricinus nymphs towards host odour is in line with the findings of Crooks and Randolph (2006) and Berret and Voordouw (2015). Ixodid tick species are known to use olfactoreceptors in their forelegs to detect and respond to $\mathrm{CO}_{2}, \mathrm{NH}_{3}$ and $\mathrm{C}_{4} \mathrm{H}_{8} \mathrm{O}_{2}$ produced by their hosts (Sonenshine et al. 1986). During questing, Ixodid ticks may not necessarily be attracted toward a passing host by the host odour, but the kairomones may be used to select a suitable questing location, preferably next to a host trail, to increase the chance to encounter a host (Carroll et al. 1995).

We, therefore, suggest that the first and last step in the host-seeking behaviour of $I$. ricinus nymphs (selection of a questing location and selection of a host or suitable feeding site on the host) is mediated by host odour. The generalist $I$. ricinus responded to a wide variety of host odours, whereas a more specialist tick species (Rhipicephalus microplus) did not respond equally to each odour source (Osterkamp et al. 1999). It is, however, unknown whether $I$. ricinus can actively select a preferred host over another host based on host odour. It is also unknown whether these effects of $\mathrm{CO}_{2}$ and host odour on I. ricinus nymphs are applicable to larvae and adult ticks. The results of (Gray 1985) indicate that all life stages respond to $\mathrm{CO}_{2}$. In another Ixodid tick species (Amblyomma variegatum), however, the effect of $\mathrm{CO}_{2}$ on tick activation and attraction varied between life stages (Barre et al. 1997). The attraction of larvae to a suitable questing location by host odours is less likely, due to their limited horizontal movements. Our results are also in line with the host-seeking behaviour of haematophagous insects, which commonly use $\mathrm{CO}_{2}$ for activation and, less often, as attractant. It is thus interesting to observe how both groups or arthropods have evolved a response to $\mathrm{CO}_{2}$ for host location. We conclude that $\mathrm{CO}_{2}$ is an activator but not an attractant and that host odour is an attractant, but not an activator of $I$. ricinus nymphs, providing ecological insights into the hostfinding behaviour of $I$. ricinus nymphs.

\section{Acknowledgements}

We thank Wouter van Andel for his help with the bioassays of experiment 2. 



\section{Chapter 8}

\section{Larvae of Ixodes ricinus transmit Borrelia afzelii and B. miyamotoi to vertebrate hosts}

Gilian van Duijvendijk, Claudia Coipan, Alex Wagemakers, Manoj Fonville, Jasmin Ersöz, Anneke Oei, Gábor Földvári, Joppe Hovius, Willem Takken, Hein Sprong 


\section{Abstract}

Background: Lyme borreliosis is the most common tick-borne human disease and is caused by Borrelia burgdorferi sensu lato (s.I.). Borrelia miyamotoi, a relapsing fever spirochaete, is transmitted transovarially, whereas this has not been shown for $B$. burgdorferi s.l. Therefore, $B$. burgdorferi s.l. is considered to cycle from nymphs to larvae through vertebrates. Larvae of Ixodes ricinus are occasionally $B$. burgdorferi s.I. infected, but their vector competence has never been studied.

Methods: We challenged 20 laboratory mice with field-collected larvae of $I$. ricinus. A subset of these larvae was analysed for infections with B. burgdorferi s.I. and B. miyamotoi. After three to four challenges, mice were sacrificed and skin and spleen samples were analysed for infection by PCR and culture.

Results: Field-collected larvae were naturally infected with B. burgdorferi s.I. $(0.62 \%)$ and $B$. miyamotoi (2.0\%). Two mice acquired a $B$. afzelii infection and four mice acquired a $B$. miyamotoi infection during the larval challenges.

Conclusion: We showed that larvae of $I$. ricinus transmit $B$. afzelii and $B$. miyamotoi to rodents and calculated that rodents have a considerable chance of acquiring infections from larvae compared to nymphs. As a result, $B$. afzelii can cycle between larvae through rodents. Our findings further imply that larval bites on humans, which easily go unnoticed, can cause Lyme borreliosis and Borrelia miyamotoi disease. 


\section{Introduction}

Lyme borreliosis is the most common vector-borne human disease in the northern hemisphere and is caused by Borrelia burgdorferi sensu lato (s.l.) (Stanek et al. 2012). Borrelia miyamotoi is an emerging pathogen which can cause relapsing fever, tentatively called Borrelia miyamotoi disease in humans (Platonov et al. 2011, Wagemakers et al. 2015). In Europe, both pathogens are transmitted by Ixodes ricinus. This tick species hatches from the egg as a larva, which feeds from a vertebrate host before moulting into a nymph. Nymphs feed again from a vertebrate host before moulting into an adult. It is generally believed that larvae of $I$. ricinus are not infected with B. burgdorferi s.I. (Richter et al. 2012, Rollend et al. 2013) and can only become infected during a blood meal from an infected host (Piesman and Sinsky 1988) or during a blood meal in the vicinity of an infected nymph feeding on an uninfected host, known as co-feeding (Gern and Rais 1996, Voordouw 2015). The infected engorged larvae then moult into infected nymphs, which can transmit the spirochaetes to new hosts (Radolf et al. 2012). Rodents are the most frequently used hosts by larvae and are reservoirs for $B$. afzelii (Kurtenbach et al. 1998b). However, B. burgdorferi s.l. is also, but rarely, found in questing larvae (Fingerle et al. 2008, Kjelland et al. 2010, Strube et al. 2010, Tappe et al. 2014). Borrelia miyamotoi, on the other hand, can be transmitted transovarially from female tick to larva (Scoles et al. 2001) but is only shortly maintained in rodents (Taylor et al. 2013a). Presence of a pathogenic microorganism in a tick does not necessarily mean that the tick is also competent as a vector (Heylen et al. 2014). On rodents, larval tick burden is much higher than nymphal tick burden, while adult $I$. ricinus rarely feed on rodents (Gassner et al. 2013). Humans are also bitten by larvae, with an estimated of at least 30,000 larval bites - out of 1.1 million tick bites in The Netherlands annually (Hofhuis 2010). Therefore, even a low B. burgdorferi s.I. infection rate in larvae will be of importance to the enzootic transmission cycle of $B$. burgdorferi s.l. (Tappe et al. 2014) and human disease risk.

The aim of this study was to examine whether the larvae of $I$. ricinus can transmit $B$. burgdorferi s.l. and B. miyamotoi to vertebrate hosts. 


\section{Methods}

\section{Study organisms}

Twenty-eight male Naval Medical Research Institute (NMRI) mice two months-old were used. Experiment 1 consisted of 10 treatment and two control mice and experiment 2 consisted of 10 treatment and six control mice. Mice were housed individually in unmodified Makrolon type II cages with ad libitum water and food. Cages were suspended over pans filled with water and with petroleum jelly on the edge.

Larvae of $I$. ricinus were captured at five locations (Buunderkamp, Grebbeberg, Sysselt, Bilderberg and Planken Wambuis) near Wageningen, The Netherlands (Table 1). All larvae were captured in June-September 2014 within two weeks prior to each challenge (see below) or pathogen detection. All locations consisted of mixed forests dominated by Scots pine (Pinus sylvestris). A $1 \times 1 \mathrm{~m}$ blanket was dragged over the vegetation (Milne 1943) and attached larvae were collected using an aspirator. A subset of these questing larvae were individually analysed for natural infections with $B$. burgdorferi s.I. and B. miyamotoi. Larvae were stored in groups of 200 in ventilated $50 \mathrm{ml}$ tubes and stored at room temperature, 90\% relative humidity $(\mathrm{RH})$ and natural day length until further use. Tick life-cycle stage was confirmed using a microscope.

Table 1. Origin, mouse numbers and infection rates of questing larvae.

\begin{tabular}{lccrr}
\hline Location & \multicolumn{1}{c}{ Coordinates } & \multicolumn{1}{c}{ Mouse } & B. burgdorferi & B. miyamotoi \\
\hline Buunderkamp & $52^{\circ} 00^{\prime} 46 " \mathrm{~N}, 5^{\circ} 45^{\prime} 42 \mathrm{E}$ & $1-2,13-14$ & $1.12(3 / 267)$ & $2.25(6 / 267)$ \\
Grebbeberg & $51^{\circ} 57^{\prime} 10^{\prime \prime} \mathrm{N}, 5^{\circ} 35^{\prime} 25 " \mathrm{E}$ & $3-4,15-16$ & $1.29(3 / 233)$ & $0.43(1 / 233)$ \\
Sysselt & $52^{\circ} 01^{\prime} 40^{\prime \prime} \mathrm{N}, 5^{\circ} 41^{\prime} 26 " \mathrm{E}$ & $5-6,17-18$ & $0.39(1 / 257)$ & $0.39(1 / 257)$ \\
Bilderberg & $51^{\circ} 59^{\prime} 55^{\prime \prime} \mathrm{N}, 5^{\circ} 48^{\prime} 34 " \mathrm{E}$ & $7-8,19-20$ & $0(0 / 362)$ & $2.49(9 / 362)$ \\
Planken Wambuis & $52^{\circ} 01^{\prime} 36^{\prime \prime} \mathrm{N}, 5^{\circ} 48^{\prime} 55^{\prime \prime} \mathrm{E}$ & $9-10,21-22$ & $0.59(2 / 337)$ & $3.56(12 / 337)$ \\
Mean & & & $0.62(9 / 1456)$ & 1.99 \\
& & & & $(29 / 1456)$ \\
\hline
\end{tabular}

Questing larvae were collected at five locations. Coordinates, mouse numbers and $B$. burgdorferi s.l. and $B$. miyamotoi infection rates (infected/analysed) of questing larvae are shown per location.

\section{Challenges with larvae}

Mice were challenged with 200 larvae on the head and neck under anaesthesia $(60 \mathrm{mg} / \mathrm{kg}$ pentobarbital, i.p.) at two-week intervals. In experiment 1, five groups of two mice were subjected to four challenges with 200 larvae collected from five different locations (Table 1). Two control mice received no larval challenge. In experiment 2, five groups of two mice were similarly subjected to three challenges with 200 field-collected larvae while six control mice were not challenged. Engorged larvae were collected from the pans with water four and seven days after each challenge and dried on filter paper for $2 \mathrm{~h}$. These larvae were then housed 
individually in ventilated $0.2 \mathrm{ml}$ Eppendorf tubes at $20^{\circ} \mathrm{C}, 90 \% \mathrm{RH}$ and a day length of $14 \mathrm{~h}$. Engorged larvae of experiment 1 were checked monthly, and 2 months after moulting, the emerged nymphs were stored at $-20^{\circ} \mathrm{C}$ until further use. Larvae that did not moult and larvae from experiment 2 were excluded from the molecular analysis.

\section{Collection of mouse tissue}

An ear biopsy was collected from each mouse with sterile scissors and tweezers one week before challenge 1 . One week after challenge 4 in experiment 1 and three weeks after challenge 3 in experiment 2, mice were sacrificed by cardiac bleed followed by cervical dislocation under anaesthesia (60 mg/kg pentobarbital, i.p.), after which two ear biopsies and a spleen sample were collected. All tissue samples were stored in $70 \%$ ethanol at $-20^{\circ} \mathrm{C}$ until further use. All experiments were approved by the Ethical committee of Wageningen University (number 2013136).

\section{Natural tick burden on field-collected rodents}

In 2013 and 2014, rodent life traps were placed with $5 \mathrm{~m}$ inter-trap distance in grids of $12 * 12$ and $6^{*} 12$, respectively, at two locations in a forest near Wageningen, The Netherlands $\left(51^{\circ} 59^{\prime} 35.43^{\prime \prime} \mathrm{N}, 5^{\circ} 43^{\prime} 42.06 " \mathrm{E}\right)$ and $\left(51^{\circ} 59^{\prime} 37.22{ }^{\prime \prime} \mathrm{N}, 5^{\circ} 43^{\prime} 22.08 " \mathrm{E}\right)$. Traps were set at 15:30h and inspected the next day at $08.30 \mathrm{~h}$ at three-week intervals from May till November. Tick burdens of trapped wood mice (Apodemus sylvaticus) and bank voles (Myodes glareolus) were determined by searching the head, ears, snout, belly, legs, armpits, throat and tail.

\section{Pathogen detection}

In experiment 2, mouse spleens and ears collected at the end of the experiment were cultured in MKP-F medium as described in detail previously (Wagemakers et al. 2014). In short: a 4 $\mathrm{mm}$ tissue biopsy was disinfected and placed in $7 \mathrm{ml}$ Modified Kelly-Pettenkofer medium containing rifampicin, fosfomycin and amphotericin $\mathrm{B}$ as antibiotics. Cultures were incubated at $33^{\circ} \mathrm{C}$ and checked weekly for motile spirochaetes using a dark-field microscope at $250 \mathrm{x}$ magnification. After three weeks $500 \mu$ of medium was inoculated into a new tube containing $7 \mathrm{ml}$ of culturing medium. This was repeated three times. DNA from the cultures containing motile spirochaetes, tissue samples and moulted nymphs were extracted using the Qiagen DNeasy Blood \& Tissue Kit (Jahfari et al. 2014). DNA from questing larvae was extracted by alkaline lysis (Schouls et al. 1999). The presence of $B$. burgdorferi s.I. DNA was detected with a duplex quantitative PCR using fragments of the outer membrane protein A gene and the flagellin B gene as targets (Hovius et al. 2013). In the same qPCR, B. miyamotoi could 
specifically be detected with primers and probe based on the flagellin gene for detection of the bacteria. The presence of Neoehrlichia mikurensis and Anaplasma phagocytophilum was detected as described (Jahfari et al. 2014). Multi-Locus Sequence Typing on the Borrelia cultures was performed as described (Coipan et al. 2013a).

\section{Data analysis}

Infection rates of questing larvae and emerged nymphs were compared using a generalized linear model (GLM, assuming binomial distribution with logit link function) in SAS statistical software, version 9.3 .

\section{Results}

Infection rates of field-collected larvae in nature and after feeding on laboratory mice A subset of 1456 field-collected questing larvae were analysed for infection. In experiment 1 , 1897 engorged field-collected larvae that fed on laboratory mice were collected, from which 1823 (96\%) moulted into nymphs. Borrelia burgdorferi s.I. infection rate increased from $0.62 \%$ $(9 / 1456)$ in questing larvae to $1.65 \%(30 / 1823)$ in larvae that moulted into nymphs after feeding (Table 1 and 2, $\mathrm{P}=0.005)$. Borrelia miyamotoi infection rate of questing larvae (1.99\%, 29/1456) and larvae that moulted into nymphs after feeding $(1.76 \%, 32 / 1823)$ did not differ $(P=0.619)$. No questing larvae were co-infected with $B$. burgdorferi s.l. and B. miyamotoi. Two nymphs (mouse 9, challenge 2 and mouse 10, challenge 4) were co-infected with $B$. burgdorferi s.l. and B. miyamotoi. In experiment 2, 2308 engorged field-collected larvae were collected, which were not further analysed. 
Table 2. Infection rates (infected/analysed) of ticks after feeding on mice of experiment 1.

\begin{tabular}{|c|c|c|c|c|c|c|}
\hline & Mouse & $\begin{array}{r}\text { Challenge } \\
1 \\
\end{array}$ & $\begin{array}{r}\text { Challenge } \\
2 \\
\end{array}$ & $\begin{array}{r}\text { Challenge } \\
3 \\
\end{array}$ & $\begin{array}{r}\text { Challenge } \\
4 \\
\end{array}$ & $\begin{array}{c}\text { PCR-positive } \\
\text { tissues }\end{array}$ \\
\hline \multirow{12}{*}{$\begin{array}{l}\bar{c} \\
\frac{0}{0} \\
\frac{0}{5} \\
\frac{0}{0} \\
\frac{0}{3} \\
0\end{array}$} & 1 & $1.3(1 / 75)$ & $0(0 / 81)$ & $2.2(1 / 45)$ & $0(0 / 48)$ & - \\
\hline & 2 & $0(0 / 32)$ & $0(0 / 39)$ & $3.8(1 / 26)$ & $0(0 / 13)$ & - \\
\hline & 3 & $1.7(1 / 60)$ & $0(0 / 64)$ & $0(0 / 34)$ & $0(0 / 34)$ & - \\
\hline & 4 & $0(0 / 47)$ & $0(0 / 31)$ & $0(0 / 32)$ & $0(0 / 26)$ & - \\
\hline & 5 & $0(0 / 80)$ & $0(0 / 47)$ & $0(0 / 55)$ & $1.3(1 / 75)$ & - \\
\hline & 6 & $0(0 / 44)$ & $0(0 / 58)$ & $0(0 / 37)$ & $0(0 / 20)$ & - \\
\hline & 7 & $0(0 / 84)$ & $0(0 / 39)$ & $0(0 / 36)$ & $0(0 / 44)$ & - \\
\hline & 8 & $2.2(1 / 45)$ & $2.1(1 / 47)$ & $0(0 / 51)$ & $0(0 / 4)$ & - \\
\hline & 9 & $0(0 / 73)$ & $2.9(1 / 35)$ & $0(0 / 15)$ & $0(0 / 21)$ & - \\
\hline & 10 & & & & 71.4 & Ear \\
\hline & & $0(0 / 102)$ & $3.2(2 / 63)$ & $0(0 / 33)$ & $(20 / 28)$ & \\
\hline & total & (3/642) & $(4 / 504)$ & $(2 / 364)$ & $(21 / 313)$ & \\
\hline \multirow{12}{*}{ 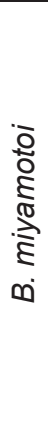 } & 1 & $0(0 / 75)$ & $2.5(2 / 81)$ & $0(0 / 45)$ & $2.1(1 / 48)$ & - \\
\hline & 2 & $0(0 / 32)$ & $7.7(3 / 39)$ & $0(0 / 26)$ & $0(0 / 13)$ & Spleen \\
\hline & 3 & $3.3(2 / 60)$ & $0(0 / 64)$ & $0(0 / 34)$ & $0(0 / 34)$ & Spleen \\
\hline & 4 & $0(0 / 47)$ & $0(0 / 31)$ & $0(0 / 32)$ & $3.8(1 / 26)$ & - \\
\hline & 5 & $1.3(1 / 80)$ & $0(0 / 47)$ & $0(0 / 55)$ & $1.3(1 / 75)$ & - \\
\hline & 6 & $0(0 / 44)$ & $0(0 / 58)$ & $2.7(1 / 37)$ & $0(0 / 20)$ & - \\
\hline & 7 & $0(0 / 84)$ & $0(0 / 39)$ & $0(0 / 36)$ & $0(0 / 44)$ & - \\
\hline & 8 & $0(0 / 45)$ & $0(0 / 47)$ & $0(0 / 51)$ & $0(0 / 4)$ & - \\
\hline & 9 & 17.8 & & & & - \\
\hline & & $(13 / 73)$ & $14.3(5 / 35)$ & $0(0 / 15)$ & $0(0 / 21)$ & \\
\hline & 10 & $0(0 / 102)$ & $0(0 / 63)$ & $3(1 / 33)$ & $3.6(1 / 28)$ & - \\
\hline & & $(16 / 642)$ & (10/504) & $(2 / 364)$ & (4/313) & \\
\hline
\end{tabular}

Last column shows PCR-positive tissues after challenge $4,-=$ uninfected

\section{Pathogen transmission by field-collected larvae}

All mice were not infected with $B$. burgdorferi s.l. and B. miyamotoi at the start of the experiments and all control mice were uninfected at the end of the experiments (Table 3). In experiment 1, one week after four challenges with field-collected larvae, 1 out of 10 mice was positive for $B$. afzelii and 2 out of 10 mice for $B$. miyamotoi. In experiment 2, three weeks after three challenges with field-collected larvae, 1 out of 10 mice was positive for both $B$. afzelii and $B$. miyamotoi. We were able to isolate and culture live spirochaetes for more than three passages from the ears of this infected mouse. The motile spirochaetes in this culture and the $B$. burgdorferi s.l.-positive tissue samples and nymphs were all identified as $B$. afzelii by molecular typing. In addition, in experiment 2, 1 out of 10 mice was positive for B. miyamotoi. None of the rodent samples were infected with Anaplasma phagocytophilum or Neoehrlichia mikurensis (data not shown). 
Table 3. Infections in mice of experiments 1 and 2.

\begin{tabular}{|c|c|c|c|c|}
\hline & Mouse & Treatment & Before challenges & After challenges \\
\hline \multirow{12}{*}{ 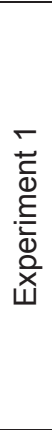 } & 1 & $\mathrm{~T}$ & - & - \\
\hline & 2 & T & - & $\mathrm{Bm}(\mathrm{S})$ \\
\hline & 3 & $\mathrm{~T}$ & - & $\mathrm{Bm}(\mathrm{S})$ \\
\hline & 4 & $\mathrm{~T}$ & - & - \\
\hline & 5 & $\mathrm{~T}$ & - & - \\
\hline & 6 & $\mathrm{~T}$ & - & - \\
\hline & 7 & $\mathrm{~T}$ & - & - \\
\hline & 8 & $\mathrm{~T}$ & - & - \\
\hline & 9 & $\mathrm{~T}$ & - & - \\
\hline & 10 & $\mathrm{~T}$ & - & $\mathrm{Ba}(\mathrm{E})$ \\
\hline & 11 & C & n.a. & - \\
\hline & 12 & C & n.a. & - \\
\hline \multirow{16}{*}{ 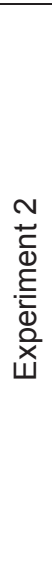 } & 13 & $\mathrm{~T}$ & - & - \\
\hline & 14 & $\mathrm{~T}$ & - & - \\
\hline & 15 & $\mathrm{~T}$ & - & - \\
\hline & 16 & $\mathrm{~T}$ & - & $\mathrm{Ba}(\mathrm{E}), \mathrm{Bm}(\mathrm{S}, \mathrm{E})$ \\
\hline & 17 & $\mathrm{~T}$ & - & - \\
\hline & 18 & $\mathrm{~T}$ & - & - \\
\hline & 19 & $\mathrm{~T}$ & - & - \\
\hline & 20 & $\mathrm{~T}$ & - & - \\
\hline & 21 & $\mathrm{~T}$ & - & - \\
\hline & 22 & $\mathrm{~T}$ & - & $\mathrm{Bm}(\mathrm{S})$ \\
\hline & 23 & C & - & - \\
\hline & 24 & C & - & - \\
\hline & 25 & C & n.a. & - \\
\hline & 26 & C & n.a. & - \\
\hline & 27 & C & n.a. & - \\
\hline & 28 & C & n.a. & - \\
\hline
\end{tabular}

$\mathrm{T}=$ treatment mouse, challenged with larvae, $\mathrm{C}=$ control mouse, $-=$ uninfected

n.a. $=$ not analysed, $\mathrm{S}=$ Spleen, $\mathrm{E}=$ Ear biopsy, $\mathrm{Ba}=$ Borrelia afzelii, $\mathrm{Bm}=$ Borrelia miyamoto $i$

\section{Natural tick burden on field-collected rodents}

In total, 335 wood mice and 521 bank voles were trapped. Total number of nymphs on these rodents was 60 (mean $0.07 \pm 0.013$ ) and total number of larvae 3157 (mean $3.69 \pm 0.235$ ), resulting in a nymph to larva ratio of 1:52.6.

\section{Discussion}

We showed that larvae of $I$. ricinus can transmit Lyme disease spirochaetes. An abstract in a conference book from 2002 claimed this finding before, but it was never published in a full research article (Hammer et al. 2002). The possibility to culture $B$. afzelii from several organs of the rodents shows that the spirochaetes were viable and infectious. The rodents infected with $B$. afzelii subsequently facilitated transmission to other larvae, which successfully moulted 
to infected nymphs ( $71.4 \%$ infectivity during the last challenge). This resulted in a higher infection rate in the emerged nymphs compared to the infection rate of questing larvae. These results are in contradiction to the general idea that $B$. burgdorferi s.l. can only be transmitted by nymphs (and adults), which acquired the infection as a larvae (or nymphs) during feeding (Piesman and Sinsky 1988, Gern and Rais 1996, Voordouw 2015). We have shown that $0.62 \%$ of the larvae in nature is infected with B. burgdorferi s.l., whereas this is about $10 \%$ (range 0 $60 \%$ ) for nymphs (Rauter and Hartung 2005, Gassner et al. 2011). On rodents, which are the main maintenance host for larvae and reservoir host of $B$. afzelii, we found a nymph to larva ratio of 1:52.6, which is in accordance with the ratios found by Gassner et al. (Gassner et al. 2013) and Pisanu et al. (Pisanu et al. 2010). This means that among 1000 feeding nymphs there are 100 infected (10\%) and at the same time 52,600 larvae feed on the same rodent population from which $326(0.62 \%)$ are infected. As a result, assuming comparable transmission efficiencies for larvae and nymphs, larval contribution to rodent infections is approximately three times higher than the nymphal contribution. When transmission efficiency from I. ricinus to rodents is considered to be $100 \%$ for nymphs (Kahl et al. 1998) and $10 \%$ for larvae (1 out of 10 larvae, table 2), larvae are still responsible for a quarter of the infections in rodents. The transmission efficiencies of larvae and nymphs need further research. Regardless of these transmission efficiencies, our results show that $B$. afzelii can also cycle between larvae through rodents without the interference of nymphs. Infection rates of $B$. burgdorferi s.I. in questing larvae can even be up to $25.8 \%$, as found in Germany (Tappe et al. 2014), increasing the importance of larval infection.

In The Netherlands, 1.1 million people are bitten by one or more ticks annually (Hofhuis 2010). Larvae are responsible for 1.3 to $4.2 \%$ of human tick bites (Fonville et al. 2013, Hofhuis et al. 2013, Lindblom et al. 2014), and it is estimated that in The Netherlands 30,000 people were bitten by larvae. A significant number of Lyme borreliosis patients did not recognize or remember being bitten by a tick, which might especially be the case for larval ticks due to their minute size (Figure 1). Our findings provide a reasonable explanation when Lyme borreliosis patients have not noticed a tick bite.

As far as we know, we also showed for the first time that larvae of $I$. ricinus can transmit $B$. miyamotoi. We found a $B$. miyamotoi infection rate of $2 \%$ in larvae, which is comparable to the infection rate found in nymphs (Richter et al. 2003, Fonville et al. 2013). This suggests a potentially higher contribution of larval tick bites to tick-borne relapsing fever in humans compared to larval contribution to Lyme borreliosis. Other studies have previously suggested an important role for larvae in the transmission of $B$. miyamotoi based on infections coinciding with larval activity and infestation (Barbour et al. 2009, Molloy et al. 2015). The contribution of larval transmission to rodents and from rodents to larvae to the enzootic life cycle of $B$. miyamotoi appears to be low. We did not find an increase in the infection rate of 


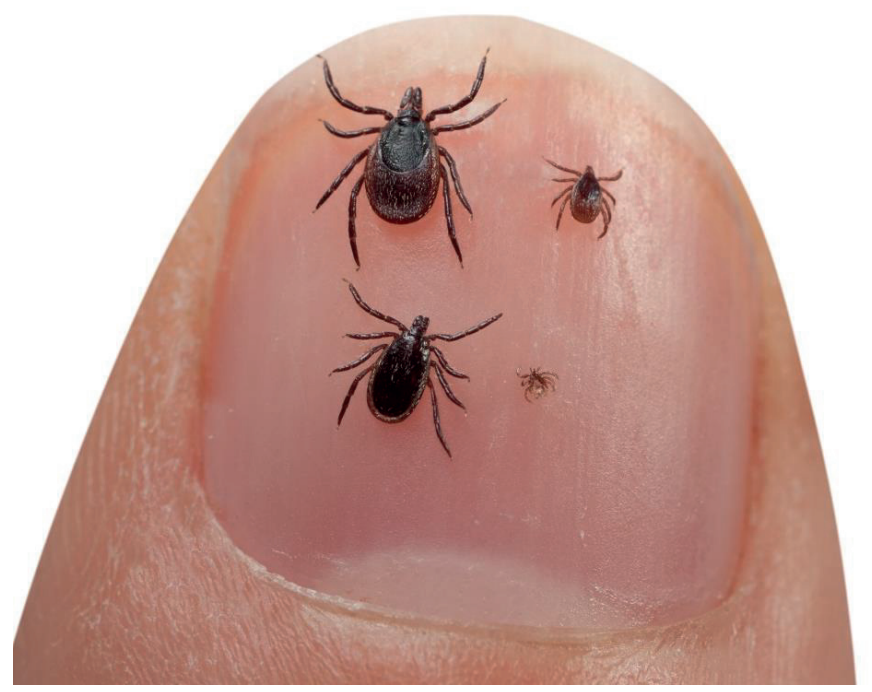

Figure 1. Developmental stages of I. ricinus. From left to right and top to bottom: Adult female, nymph, adult male, larva (Picture: Hans Smid).

larvae with successive challenges, even in the two mice (2 and 3) that were B. miyamotoi positive after challenge 4 . Infection rate of the emerged nymphs was not higher compared to the infection rate in questing larvae. In addition, mouse 9 was infectious to feeding larvae during challenge 1 and challenge 2, but was PCR negative after challenge 4 , suggesting that, in contrast to $B$. afzelii, B. miyamotoi does not cause a persistent infection in rodents (Taylor et al. 2013a).

In experiment 1, 7 out of 10 mice were exposed to larvae that were (after feeding) $B$. afzeliipositive and 9 out of 10 mice to $B$. miyamotoi-positive larvae. However, only 1 out of 10 and 2 out of 10 mice were positive after challenge 4 for $B$. afzelii and $B$ miyamotoi, respectively. For $B$. afzelii, this can indicate a low transmission efficiency from larva to rodent, whereas the $B$. miyamotoi infections could have been cleared from the rodents before the dissections after challenge 4 . In addition, mice were only naïve during the first challenge because they were challenged 4 times with larvae. This could have resulted in the production of antibodies against B. miyamotoi making the mice resistant to infections during challenge 4 .

Knowing now that larvae of $I$. ricinus can transmit $B$. afzelii to rodents, the next question is how larvae can acquire the infection. In theory, larvae may acquire spirochaetes through transovarial transmission from female tick to larvae or through a partial blood meal from a rodent, after which the larva quests for a second host. Transovarial transmission, however, has never been demonstrated for B. burgdorferi s.l. but is indeed considered an important mechanism for B. miyamotoi (Scoles et al. 2001, Richter et al. 2012, Rollend et al. 2013). Partial feeding on an infected rodent for only eight hours incidentally resulted in $B$. burgdorferi 
S.I. infection of $I$. scapularis larvae (Piesman 1991). In addition, nymphs that have fed for up to $48 \mathrm{~h}$ on one host can thereafter infect another host without first moulting into an adult (Shih and Spielman 1993). Partially engorged larvae, however, were unable to transmit $B$. burgdorferi s.l. to a host during a subsequent feeding, which was argued to be because of a too low spirochete load for efficient transmission (Piesman 1991). However, an inoculum of only 10 spirochaetes has been demonstrated to be sufficient to successfully infect a rodent (Barthold 1991). Partially-engorged larvae can arise when feeding larvae are detached due to grooming or due to an immune response or death of the host (Trager 1939, Levin and Fish 1998, Brossard and Wikel 2004). Larvae appear to be unfed macroscopically after up to $18 \mathrm{~h}$ of feeding, during which they may have acquired an infection (Piesman 1991). Partiallyengorged questing nymphs are occasionally observed in the field (GD, pers. obs.). However, frequency of naturally occurring partially engorged and B. burgdorferi s.l.-infected larvae is unknown. Both mechanisms (transovarial transmission and partial feeding) seem equally unlikely. The route via which larvae can acquire a $B$. burgdorferi s.l. infection needs further research.

\section{Conclusions}

Larvae of $I$. ricinus can transmit $B$. afzelii and $B$. miyamotoi. This finding changes the current view on the enzootic lifecycle of $B$. afzelii and the current public health dogma that larval bites cannot cause Lyme disease in humans.

\section{Acknowledgements}

We thank Marloes van Schaijk and Inge Krijger for their help with the rodent trapping. 



\section{Chapter 9}

General discussion 
General discussion 


\section{Introduction}

The aim of this thesis was to gain insight in the multi-trophic interactions that affect the density of $B$. burgdorferi s.l.-infected and B. miyamotoi-infected $I$. ricinus nymphs. As in The Netherlands $B$. afzelii is the most prevalent $B$. burgdorferi s.l. genospecies (Gassner et al. 2011), this thesis focuses on $B$. afzelii as a proxy for $B$. burgdorferi s.l. infections.

The development from uninfected questing larva to $B$. afzelii-infected questing nymph is one of the key factors affecting the density of infected nymphs (DIN) and was dissected in four steps: 1) larval host attachment, 2) larval blood feeding, 3) spirochaete transmission from rodent to larva, and 4) development from engorged larva to nymph (Figure 1, Chapter 2). Rodents acquire $B$. burgdorferi s.I. spirochaetes through the bite of an infected tick. Nymphal tick burden was, therefore, also included in this thesis because this closes the $B$. afzelii life cycle, affects rodent infection rate and consequently DIN. A review of the literature showed that the ecology of $B$. afzelii is complex and that there are several knowledge gaps, some of which I aimed to fill with the experiments described in this thesis. Experimental evidence of the effect of rodent density on DIN, host-seeking behaviour of $I$. ricinus, the effect of a $B$. afzelii infection on its own transmission and proof of the vector competence of $I$. ricinus larvae were lacking. The ecology of $B$. miyamotoi is relatively unknown and was included in two experimental chapters.

The main conclusions of this thesis are:

1) Rodent density affects the density of $B$. burgdorferi s.I.-infected nymphs one year later when questing larvae are not the limiting factor.

2) The aggregation of $I$. ricinus larvae on rodents is caused by variation in host characteristics (e.g. species, sex, body weight).

3) Wood mice have larger tick burdens and larvae take a larger blood meal from wood mice compared to bank voles.

4) I. ricinus is activated by $\mathrm{CO}_{2}$ and attracted to host odour.

5) $B$. afzelii increases larval tick burden on bank voles and nymphal body weight of $I$. ricinus.

6) I. ricinus larvae can transmit $B$. afzelii and $B$. miyamotoi to rodents.

These results are discussed below. 


\section{Life cycle of $B$. afzelii and $B$. miyamotoi}

\section{Role of rodents}

It is generally assumed that wood mice and bank voles can only acquire a $B$. afzelii infection via the bite of an infected nymph because adult ticks rarely feed on rodents and questing larvae are thought to be free of B. burgdorferi s.I. (Richter et al. 2012, Rollend et al. 2013). Infected rodents transmit the spirochaetes to feeding larvae, which in turn moult into infected nymphs, closing the $B$. afzelii lifecycle (Radolf et al. 2012). There are, however, occasional records of B. burgdorferi s.l.-infected larvae (Fingerle et al. 2008, Kjelland et al. 2010, Strube et al. 2010, Tappe et al. 2014), but it is unknown whether these larvae are able to transmit the spirochaetes to a host. It was, therefore, investigated whether larvae can infect rodents with $B$. afzelii. I found that questing larvae collected from natural areas were infected with $B$. afzelii and transmitted the spirochaetes to rodents (Van Duijvendijk et al. 2016). This finding is the first definite proof that $I$. ricinus larvae can be naturally infected with $B$. afzelii. Less surprising, but still novel, the experiment also shows that questing larvae can transmit $B$. miyamotoi to rodents. This spirochaete was shown to be transmitted transovarially from female tick to larva via the egg (Scoles et al. 2001), which is thought not to be the case for B. burgdorferi s.l. (Richter et al. 2012, Rollend et al. 2013). The role of rodents in the life cycle of B. miyamotoi, however, seems to be limited because rodents can clear a B. miyamotoi infection (Taylor et al. 2013, Wagemakers et al. 2016) and because the $B$. miyamotoi infection rate hardly increases from larva to nymph to adult (Heylen et al. 2016, Paul et al. 2016, Siński et al. 2016, Sormunen et al. 2016, Van Duijvendijk et al. 2016). The B. afzelii infection rate of field-collected questing larvae was low. I calculated, however, that, due to the high number of larvae feeding on rodents compared to the number of rodent-feeding nymphs, larvae contribute significantly to the life cycle of $B$. afzelii by being responsible for about $25-75 \%$ of the infections in rodents. This knowledge changes the current view on the enzootic $B$. afzelii life cycle. It is, however, unknown through which process $I$. ricinus larvae acquired the $B$. afzelii infection. There are two hypotheses for this. The first hypothesis is that $B$. afzelii can be transmitted transovarially from female tick to larva as was shown for $B$. miyamotoi although it remains a rare event, as proposed by Richter et al. (2012). The reason why this may not have been found previously could be that almost all female ticks feed on Borrelia-incompetent hosts that kill the spirochaetes in the female tick during feeding (Pacilly et al. 2014, Hofmeester et al. 2016). When female ticks feed occasionally on hosts that do not clear the $B$. afzelii infection in the tick, transovarial transmission could be possible resulting in a low $B$. afzelii infection rate in questing larvae. The effects of the complement from $B$. afzelii-incompetent hosts on the survival of $B$. miyamotoi in the tick during feeding is, however, unknown and needs further 
research to clarify why the infection rate of larvae is higher for $B$. miyamotoi compared to $B$. afzelii. A second hypothesis is that $I$. ricinus larvae can acquire a partial blood meal from an infected rodent, acquire the infection, but continue to quest for a second host without first moulting to a nymph (see Chapter 8). The knowledge that $I$. ricinus larvae can transmit $B$. afzelii to rodents was applied during the interpretation of the results from the field experiments of this thesis (see below).

Lyme borreliosis is caused by different genospecies from the $B$. burgdorferi s.l. complex. The molecular analysis used in the field studies of this thesis did, however, not distinguish between the different $B$. burgdorferi s.I. genospecies. In western Europe, rodents and larvae that had fed on rodents are mainly infected with B. afzelii (Humair et al. 1999, Hanincová et al. 2003a, Kybicova et al. 2008, Schmidt et al. 2014b). The effect of rodent density on the density of infected nymphs is, therefore, assumed to differ between $B$. burgdorferi s.l. genospecies and to be most positive for $B$. afzelii. Rodents can, however, also be infected with other genospecies of the $B$. burgdorferi s.I. complex; B. bavariensis B. burgdorferi sensu stricto and Borrelia spielmanii, but their infection rates in rodents and ticks are lower (Kurtenbach et al. 1994, Humair et al. 1995, Gassner et al. 2011, Richter et al. 2011, Coipan et al. 2016). As discussed above, for now in this discussion, I will assume that the $B$. burgdorferi s.l. infections in rodents and ticks collected from rodents that were analysed in this thesis were $B$. afzelii because this is the most common genospecies associated to rodents.

In The Netherlands, wood mice and bank voles are the most commonly used hosts of $I$. ricinus larvae (Gassner et al. 2013, Hofmeester et al. 2016) as well as hosts for B. afzelii (Kurtenbach et al. 2002, Hanincová et al. 2003a). Borrelia afzelii is the most common genospecies in questing nymphs in western Europe (Gassner et al. 2011, Wilhelmsson et al. 2013, Coipan et al. 2016), underlining the large contribution of rodents to the blood meals of tick larvae and Lyme borreliosis risk. Borrelia miyamotoi can also circulate between rodents and $I$. ricinus, but the importance of rodents for the $B$. miyamotoi lifecycle is less understood because this spirochaete species is transmitted transovarially from infected female ticks to larvae and because infected rodents can clear the infection (Scoles et al. 2001, Taylor et al. 2013, Burri et al. 2014, Cosson et al. 2014, Szekeres et al. 2015, Wagemakers et al. 2015). Because rodents are the main blood hosts of $I$. ricinus larvae, rodent density was assumed to have an effect on density of infected nymphs one year later. This hypothesis was tested in two different field experiments and supported one of these experiments. One experiment, in which rodent density was artificially manipulated, showed a positive effect of rodent density on the density of $B$. afzelii-infected nymphs one year later (Chapter 3 ). This effect was caused by an increased host encounter rate of questing larvae, which resulted in a higher density of infected 
nymphs one year later. Nymphal infection prevalence was, however, not affected. The results of the other field experiment show only a weak effect of rodent density on the density of $B$. burgdorferi s.l.-infected nymphs (Chapter 4 ). This weak effect was probably caused by a dilution effect in larval and nymphal tick burdens on rodents, which resulted in a reduced exposure to $B$. burgdorferi s.l. and, therefore, a lower rodent infection rate. This lower rodent infection rate reduced nymphal infection prevalence and, consequently, affected the density of infected nymphs. In this experiment, $B$. miyamotoi infections were also analysed and the number of $B$. miyamotoi-infected nymphs yielded by the total rodent population was not affected by rodent density. There may be two factors that affected this difference in the effect of rodent density on the density of $B$. afzelii-infected nymphs between both experiments. I assume that the density of $B$. afzelii-infected nymphs depends on two major factors; 1) the density of questing larvae in the previous year and 2) the density of infected rodents in the previous year (Figure 1). In addition, I assume that the density of B. miyamotoi-infected nymphs is affected by the same two major factors but because $B$. miyamotoi infection rate hardly increases from questing larvae to nymphs I assume that larval infection prevalence in the previous year also has an effect on the density of $B$. miyamotoi-infected nymphs. The direct effect of larval infection prevalence on the density of $B$. afzelii infected nymphs is much smaller due to its low infection prevalence in larvae and high infection prevalence in nymphs, which acquired the infections from rodents. The indirect effect of larval infection prevalence (by affecting rodent infection rate) is assumed to be higher for $B$. afzelii compared to $B$. miyamotoi. This schematic overview helps to explain the differences in the effect of rodent density on the density of $B$. afzelii-infected nymphs between the experiments.
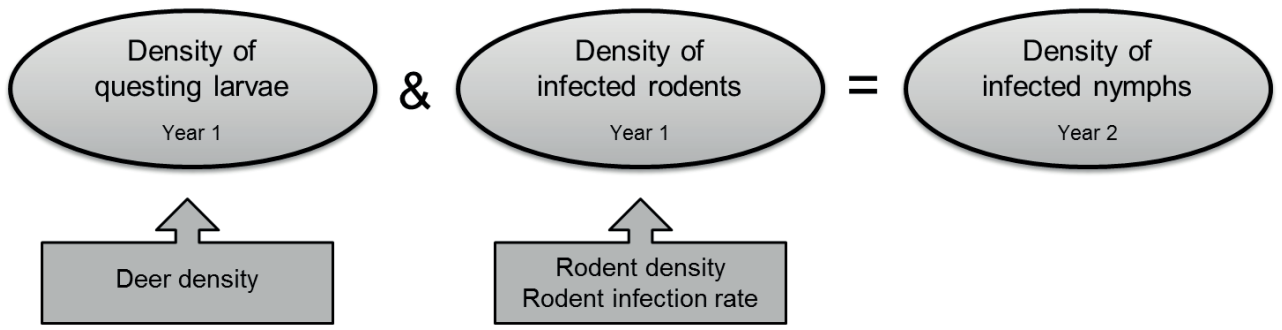

Figure 1. Schematic overview of the major ecological factors that affect the density of $B$. afzeliiinfected nymphs.

The density of $B$. afzelii-infected nymphs and density of $B$. miyamotoi-infected nymphs can only be affected by the density of rodents when the density of infected rodents, and not the density of questing larvae is the limiting factor determining the density of infected nymphs. The density of questing larvae is affected by the density of deer (Ostfeld et al. 2006, Radolf et al. 
2012), because deer are hosts for adult ticks. The factors that affect larval infection prevalence with $B$. miyamotoi, which is assumed to affect the density of $B$. miyamotoi-infected nymphs, are unclear (Siński et al. 2016), but the complement of the hosts of adult female ticks may have an effect in the infection status of the ticks, as was shown for B. burgdorferi s.l. (Pacilly et al. 2014).

The density of infected rodents depends on rodent density, which is affected by food, shelter, predation and climate (Zwolak et al. 2016), and rodent infection rate, which is affected by the exposure of rodents to infected ticks. I assume that rodent density was the limiting factor in the experiment that showed an effect of rodent density on the density of infected nymphs, whereas the density of larvae was the limiting factor in the experiment where rodent density had only a small effect on the density of infected nymphs. The study area of the experiment that showed that rodent density affected the density of questing nymphs one year later (Chapter 3 ) has a high density of roe deer, red deer and harbours large herds of cattle and horses, whereas rodent density was low due to a relatively poor vegetation dominated by scotch pine and little undergrowth. The study area where rodent density hardly affected the density of nymphs one year later (Chapter 4), however, has a low density of roe deer, whereas rodent density was high due to more undergrowth, dead wood, and masting trees in the environment. The relationship that was found between rodent density and density of infected nymphs one year later was not linear and flattened out at high rodent densities (Figure 2A Chapter 3), supporting the importance of the balance between the density of questing larvae and density of infected rodents. It was shown that the effect of deer density on the density of nymphs also flattened out at high deer density (Levi et al. 2012), supporting the importance of a balance between all factors that affect the density of infected nymphs. Due to the abundance of blackberry (Rubus fruticosus), it was not possible to determine the density of questing larvae and nymphs in the research plots of one of the experiments. Furthermore, a difference in rodent trapping method prevents a direct comparison of rodent densities between the experiments. The density of questing larvae and the density of infected rodents can, therefore, not be compared between both experiments to test these assumptions.

Not only the effects of rodent density on the density of infected nymphs one year later varied between the experiments. The effect of rodent density on larval and nymphal tick burden also varied between the experiments. Rodent density had a positive effect on tick burden in two out of three years in the experiment where rodent density had a positive effect on the density of questing nymphs one year later, whereas rodent density had a negative effect on tick burden in the experiment where rodent density hardly affected the density on infected nymphs one year later. Because rodents acquire their infections from larvae and nymphs (Radolf et al. 2012, Van Duijvendijk et al. 2016), tick burden would positively affect rodent infection rate. 
Overall, this effect of tick burden on rodent infection rate was confirmed by the results of the field experiments. Larval and nymphal tick burden are correlated, making it difficult to determine which developmental stage contributed most to the decrease in rodent infection rate when tick burdens decreased. It is unknown what caused the difference in the effect of rodent density on tick burden between the experiments. A difference between the two experiments was the scale at which rodent density varied. In the first experiment, where rodent density had a magnifying effect on tick burden, rodent density varied spatially. In the second experiment, where rodent density had a diluting effect on tick burden, rodent density varied temporally. Even though the treatments had an effect on rodent density, the natural spatial variation in rodent density between plots could have corresponded to the natural variations in deer density and, therefore, density of questing larvae. A higher rodent density could then result in a higher tick burden. The natural temporal variation in rodent density between years was unlikely to correspond to the variations in deer density because the lifespan of deer is much longer than the lifespan of rodents and their density was, therefore, unlikely to be affected by the environmental factors (mast seed availability and winter temperature and duration) than the density of rodents. This, however, needs further research to clarify this difference more clearly.

To conclude, the effect of rodent density on the density of infected nymphs depends on the balance between the density of larvae and the density of infected rodents. Rodent density affects the density of infected nymphs when rodent density is the limiting factor, but the effect is abolished due to a reduced larval tick burden, rodent infection rate and nymphal infection prevalence when the density of questing larvae is the limiting factor.

\section{Variation between rodents.}

The data from the field experiments show that rodent density is lowest in March due to a high winter mortality, and increases from spring till it reaches its peak at the end of autumn, after which reproduction decreases with an increased mortality during winter. The peak in rodent density does not entirely overlap with the peak in larval tick burden and rodent infection rate, which occur during the summer months. The contribution of the rodent population to the acarological risk in the next year appears to be highest during summer. Even though rodent density is higher in the autumn, the peaks in rodent infection rate and larval tick burden during summer make up for the lower rodent density in summer.

The aggregation of larvae on a small fraction of the wood mouse and bank vole populations causes that the contribution of the rodent population to Lyme borreliosis risk is ascribed to only about $20 \%$ of the rodent population. This aggregation of ticks on rodents contributes to the $B$. afzelii lifecycle (Van Duijvendijk et al. 2015) and is caused by characteristics of the rodents. 
Overall, Lyme borreliosis risk was most affected by the density of male wood mice, with a large body weight during summer months of the previous year.

\section{Host seeking and host selection}

Ixodes ricinus is a generalist tick species that feeds on a variety of vertebrate host species (Mannelli et al. 2012). The different developmental stages of this tick species may, however, be more specialist feeders. Larvae feed mainly on rodents, nymphs feed on a variety of vertebrate species and adult females feed mainly on large mammals (Hofmeester et al. 2016). The mechanism that causes this host specialisation is not clear. It may be caused by the behaviour of the host or tick. Environmental temperature is a major factor affecting questing behaviour of ticks; they start questing when the maximum day temperature exceeds $7^{\circ} \mathrm{C}$ (Perret et al. 2000). During questing, I. ricinus sits and waits in the vegetation for passing hosts. Larvae quest lower to the ground compared to nymphs (Mejlon and Jaenson 1997), encountering more rodents, which forage in the leaf litter. Questing location of ticks may be determined by host odour on the vegetation, as was shown for adult ticks and deer odour (Carroll et al. 1995) and nymphs and rodent odour (Berret and Voordouw 2015). Ixodes scapularis and I. pacificus larvae preferred the odours from mice compared to that from chipmunks and chicken (James and Oliver Jr 1990, Shaw et al. 2003) and this host preference may differ between the developmental stages of the tick (Dallas and Fore 2013). Ticks can also show a preference for one host over another within a certain species, like males over females (Dallas and Fore 2013). Most behavioural studies with ticks analysed host preference in a two-choice bioassay. In a natural situation, however, it is unlikely for the ticks to have a choice between two simultaneously passing hosts and they may not have a choice. It is unknown whether questing ticks do not attach to a passing, non-favoured, host, risking the chance not to encounter any other host at all. In addition, the cues that affect host seeking behaviour of $I$. ricinus are not well understood. Host odour and $\mathrm{CO}_{2}$ are commonly used cues in host-seeking behaviour of ticks and haematophagous insects (Garcia 1962, Takken and Kline 1989, Koenraadt and Dicke 2010, Chaisson and Hallem 2012, Webster et al. 2015), but heir differential effect on the host-seeking behaviour of $I$. ricinus has never been clarified. I, therefore, investigated the effect of $\mathrm{CO}_{2}$ and bank vole odour on the host seeking-behaviour of $I$. ricinus nymphs. This study showed that $I$. ricinus nymphs are activated by $\mathrm{CO}_{2}$ but that this compound did not attract them. $\mathrm{CO}_{2}$ is a waste product of metabolism and is produced by all vertebrate host species that are used by I. ricinus. Ixodes ricinus was, however, attracted to bank vole odour. Ticks may, therefore, use host odour for the selection of a questing location and then wait in the vegetation until they detect $\mathrm{CO}_{2}$ produced by the host and may then select their host using host odour. $\mathrm{CO}_{2}$ and host odour are, however, not essential for a response in 
ticks; dragging a cotton blanket over the vegetation triggers questing ticks to hold on too, suggesting that a mechanical cue can also induce a host contact response.

It is known that host species can differ in their suitability as host for I. scapularis affecting blood meal size and moulting success (Brunner et al. 2011). I investigated the effect of rodent species on blood meal size of $I$. ricinus larvae and showed that wood mice are better hosts than bank voles. It would be advantageous for the tick to select the best rodent species to ensure optimal fitness. Whether $I$. ricinus larvae have a preference for rodents over deer or wood mice of bank voles is, however, unknown.

Borrelia infections also influence the odours of their mammalian hosts. I found that, using a two-choice behavioural bioassay, I. ricinus larvae and nymphs preferred the odours from $B$. afzelii-infected rodents over uninfected rodents under semi-field conditions and in a laboratory setup. This $B$. afzelii mediated host preference of ticks has implications for the $B$. afzelii lifecycle, which I will show with the following simplistic calculations. The infectiousness of bank voles was $28.2 \%$ (29 out of 103 larvae; Figure 3, Chapter 5). In a natural situation, the infection rate of bank voles is, on average, 23\% (434/1912) (Humair et al. 1995, Kurtenbach et al. 1998b, Gray et al. 1999, Humair et al. 1999, Hanincová et al. 2003a, Richter and Matuschka 2006, Hellgren et al. 2011, Perez et al. 2012, Raberg 2012, Gassner et al. 2013, Marsot et al. 2013, Radzijevskaja et al. 2013, Schmidt et al. 2014a). In a situation where a B. afzelii infection would not increase larval tick burden, the ratio of larval tick burdens on uninfected and infected bank voles would be $0.5: 0.5$. Consequently, the ratio of larval tick burdens on the population of uninfected and the population of infected bank voles would be $0.77: 0.23$. Assuming that moulting success is not influenced by the infection, this would result in a nymphal infection rate of $6.49 \%\left(0.23^{*} 28.2\right)$. However, assuming equal sex ratios, the ratio of larval tick burdens on uninfected and infected bank voles was 0.42:0.58 (mean of 25.1 and 34.7 larvae per rodent, respectively). Therefore, in the current situation, where a $B$. afzelii infection leads to increased tick burden, the ratio of larval tick burdens in a natural population with an infection rate of $23 \%$ would be $0.71: 0.29\left(\left(0.42^{*} 0.77\right):\left(0.58^{*} 0.23\right)\right)$ for uninfected and infected bank voles. Assuming that infectivity is not influenced by tick burden, this would result in a nymphal infection rate of $8.24 \%\left(0.29^{*} 28.2\right)$. As a result, by increasing larval tick burdens with $38.2 \%((0.58-$ $0.42) / 0.42 * 100)$, the $B$. afzelii infection rate in questing nymphs that fed as larvae on bank voles increases with $27 \%((7.58-5.92) / 5.92 * 100)$. It was shown that about $70 \%$ of the $B$. burgdorferi s.l. infected nymphs is infected with $B$. afzelii (Gassner et al. 2011), resulting in 15,400 humans (of the 22,000 B. burgdorferi s.l. cases) infected with $B$. afzelii in The Netherlands in 2009 (Hofhuis et al. 2015). From these patients, 3300 (15400-(15400/1.27)) are the result of the $27 \%$ increased nymphal infection prevalence with $B$. afzelii. The increase in nymphal infection prevalence would also result in an increase in rodent infection rate, 
enlarging the effects of the larger larval tick burdens on infected rodents on Lyme borreliosis risk.

In addition to the increased tick burden on infected bank voles, the body weight of infected nymphs was $10 \%((0.198-0.180) / 0.180$, Figure 3, Chapter 5$)$ higher than the body weight of uninfected nymphs. This is in line with the $12 \%$ increased fat content of infected questing nymphs collected from the vegetation (Herrmann et al. 2013b). If a $12 \%$ higher fat content results in a $12 \%$ higher density of infected nymphs, this could not only have a direct effect on Lyme borreliosis risk, but it could also have an indirect effect on nymphal infection prevalence by increasing rodent infection rate. Further research is needed to determine how the increased nymphal infection prevalence and nymphal fat storage affect Lyme borreliosis risk.

It is not clear whether the increased tick burden on infected bank voles and increased body weight of infected nymphs are examples of true host and vector manipulation. The results from my experiments may have been caused by the infection, but may also have been caused by an infection in general and therefore, not be parasite specific. Host manipulation is a common strategy of parasites to alter host behaviour to enhance parasite fitness by e.g. increasing transmission to the next host (Hurd 2003, Lefèvre and Thomas 2008, De Moraes et al. 2014, Poulin and Maure 2015). Further research is needed to determine whether the increased tick burden on infected rodents is $B$. afzelii specific or whether this is also caused by parasitic infections that do not benefit from an increased tick burden on infected rodents. Whether the larger tick burden on infected rodents and the larger body weight of emerged nymphs is pathogen specific or not, the life cycle of $B$. afzelii benefits from these effects. A higher tick burden on infected rodents was also found in the field (Gassner 2010). I also tested whether a $B$. burgdorferi s.I. infection in field-collected rodents had an effect on their tick burden and found that infected rodents had a higher larval tick burden than uninfected rodents when rodent weight was excluded from the model (data not shown). Tick burden was, however, not affected by a $B$. burgdorferi s.I. infection in the rodent when rodent weight was excluded from the model. This was probably caused by the positive effect of rodent weight on both tick burden and rodent infection rate. Rodents cannot lose a B. afzelii infection (Richter et al. 2004a) and, in general, older rodents have a larger body weight than younger ones. Rodent weight had no effect on the results of the laboratory experiment. It is unclear why the effect of a $B$. burgdorferi s.I. infection on tick burden varied between field and laboratory conditions. A possible explanation is that in a natural situation, rodents may be infected with multiple parasites, which may affect their ability to manipulate their host (Hafer and Milinski 2015).

For the spirochaetes, a larger tick burden on infected rodents enhances transmission from rodent to tick. There is also evidence that a B. burgdorferi s.I. infection affects tick behaviour (Lefcort and Durden 1996, Herrmann and Gern 2015). The life cycle of the spirochaete also depends on the chance that an infected tick feeds on a Borrelia-competent host. The effect of 
a $B$. burgdorferi s.I. infection on nymphal behaviour was tested in a Y-tube olfactometer, but due to a low response and low nymphal infection prevalence I did not find any effects (data not shown). For $B$. afzelii it would be advantageous when an infected nymph would feed on a rodent, whereas it would be disadvantageous for $B$. garinii, because rodents are incompetent host for B. garnii, which is related to birds (Kurtenbach et al. 2002a, Hanincová et al. 2003a, Hanincová et al. 2003b). Thus, host mediation by a parasite like B. burgdorferi s.l. seems likely to be parasite-species dependent. Furthermore, I also showed that the $B$. afzelii infected hosts were better hosts for ticks, but that this was only the case when the larvae acquired the infection from the host, enabling them to take a larger blood meal (see above). It is assumed that a larger blood meal enhances survival, because this is the only nutrient uptake of the tick (Herrmann and Gern 2010). If ticks can select their preferred host based on odour, they may prefer $B$. afzelii-infected rodents over uninfected rodents to enlarge their chance of acquiring a large blood meal. Not all larvae that fed on a $B$. afzelii infected rodent, however, acquired the infection. The factors that determine whether a larvae feeding on a rodent acquires the infection are unknown. The infection process is possibly influenced by the feeding location of the tick. Ticks, do exhibit a preference for certain feeding locations of the host over others (Gassner 2010, Mysterud et al. 2014), which may be based on environmental cues like odour and temperature.

\section{Future research}

The results of this thesis suggest that it is theoretically possible to reduce the incidence of Lyme borreliosis by removing rodents until a certain threshold has been reached. The height of this threshold depends on the density of questing ticks. The results also underline that $I$. ricinus larvae are aggregated on a small fraction of the rodent population. Regarding interventions, the results of this study would support the removal of heavy bodied adult male wood mice during summer. It is however, unknown how this will affect the heterogeneity of larvae on the remaining rodents. In addition, and perhaps even more relevant, even though the removal of these rodents may reduce Lyme borreliosis risk, control of rodent populations, especially targeting rodents with specific characteristics (body size, sex, species) is complex, time consuming and ethically questionable. I also indicated that the number of questing larvae is an important factor regulating Lyme borreliosis risk. Another option for the desired reduction the density of infected nymphs would be to remove the dominant hosts of adult ticks, like roe deer and red deer. This may be more feasible due to the lower numbers of these animals, but ethically questionable too.

Two relevant fundamental research questions for follow up research on the results of this thesis are; 1 ) How can questing $I$. ricinus larvae become infected with $B$. afzelii? and 2 ) Is the 
increased tick burden on infected rodents and increased nymphal body weight of infected ticks caused by pathogen specific manipulation? These questions are the topics of current research. Another area that requires more research is in depth knowledge of the rodent body and its effect on spirochaete transmission to feeding larvae. I first looked at the effects of the entire rodent population on Lyme borreliosis risk and then studied in more detail the specific characteristics of the rodents that contributed the most. Zooming in a little further helps understanding the multi-trophic interactions that affect the density of $B$. afzelii-infected and $B$. miyamotoi-infected I. ricinus nymphs. There is, for example, not much known about the effects of e.g. feeding location of the tick on e.g. blood meal success of feeding larvae and spirochaete transmission from rodent tick larvae. The effect of rodent density on the composition of the different $B$. burgdorferi s.l. genospecies in questing nymphs and on the density of $B$. miyamotoi-infected nymphs is also a topic of current research.

In addition to the ecological studies on the interactions between ticks, rodents and $B$. burgdorferi s.I. and B. miyamotoi, I spent a considerable amount of time investigating the possibility of controlling ticks with entomopathogenic fungi in the search for an effective intervention that does not require chemical pesticides. These data are not presented in this thesis and will be published elsewhere. The three different species of entomopathogenic fungi (Beauveria bassiana, Metarhizium anisopliae and Verticillium lecanii) that were tested at different concentrations, temperatures and with varying exposure methods did not affect the survival of different life stages of $I$. ricinus.

This thesis increased our understanding of the interactions that determine the ecology of $I$. ricinus, $B$. afzelii and $B$. miyamotoi. The knowledge gained may be used to develop intervention tools that aim to reduce the incidence of Lyme borreliosis. 



\section{References}


Alani, A. J., and I. V. Herbert. 1987. Effect of host resistance on the feeding and reproductive performance of Haemaphysalis punctata and Ixodes ricinus ticks. Res Vet Sci 42: 238243.

Alugupalli, K. R., A. D. Michelson, I. Joris, T. G. Schwan, K. Hodivala-Dilke, R. O. Hynes, and J. M. Leong. 2003. Spirochete-platelet attachment and thrombocytopenia in murine relapsing fever borreliosis. Blood 102: 2843-2850.

Barandika, J. F., A. Hurtado, R. A. Juste, and A. L. García-Pérez. 2010. Seasonal dynamics of Ixodes ricinus in a 3-year period in northern Spain: First survey on the presence of tick-borne encephalitis virus. Vector Borne Zoonotic Dis. 10: 1027-1035.

Barandika, J. F., E. Berriatua, M. Barral, R. A. Juste, P. Anda, and A. L. García-Pérez. 2006. Risk factors associated with ixodid tick species distributions in the Basque region in Spain. Med. Vet. Entomol. 20: 177-188.

Barbour, A. G., J. Bunikis, D. Fish, and K. Hanincová. 2015. Association between body size and reservoir competence of mammals bearing Borrelia burgdorferi at an endemic site in the northeastern United States. Parasit Vectors 8: 299.

Barbour, A. G., J. Bunikis, B. Travinsky, A. G. Hoen, M. A. Diuk-Wasser, D. Fish, and J. I. Tsao. 2009. Niche partitioning of Borrelia burgdorferi and Borrelia miyamotoi in the same tick vector and mammalian reservoir species. Am. J. Trop. Med. Hyg. 81: 11201131.

Barre, N., G. I. Garris, and O. Lorvelec. 1997. Field sampling of the tick Amblyomma variegatum (Acari: Ixodidae) on pastures in Guadeloupe; attraction of $\mathrm{CO}_{2}$ and/or tick pheromones and conditions of use. Exp. Appl. Acarol. 21: 95-108.

Barthélémy, M., C. Gabrion, and G. Petit. 2005. Does chronic malaria modify the odours of its male mouse host? Can J Zool 83: 1079-1086.

Barthold, S. W. 1991. Infectivity of Borrelia burgdorferi relative to route of inoculation and genotype in laboratory mice. J. Infect. Dis. 163: 419-420.

Berret, J., and M. J. Voordouw. 2015. Lyme disease bacterium does not affect attraction to rodent odour in the tick vector. Parasit Vectors 8: 249.

Bize, P., C. Jeanneret, A. Klopfenstein, and A. Roulin. 2008. What makes a host profitable? Parasites balance host nutritive resources against immunity. Am. Nat. 171: 107-118.

Bogdziewicz, M., and J. Szymkowiak. 2016. Oak acorn crop and Google search volume predict Lyme disease risk in temperate Europe. Basic Appl. Ecol. 17: 300-307.

Bogdziewicz, M., R. Zwolak, and E. E. Crone. 2016. How do vertebrates respond to mast seeding? Oikos 125: 300-307.

Bown, K. J., X. Lambin, G. R. Telford, N. H. Ogden, S. Telfer, Z. Woldehiwet, and R. J. Birtles. 2008. Relative importance of Ixodes ricinus and Ixodes trianguliceps as vectors 
for Anaplasma phagocytophilum and Babesia microti in field vole (Microtus agrestis) populations. Appl. Environ. Microbiol. 74: 7118-7125.

Boyard, C., G. Vourc'h, and J. Barnouin. 2008. The relationships between Ixodes ricinus and small mammal species at the woodland-pasture interface. Exp. Appl. Acarol. 44: 61-76.

Brisson, D., D. E. Dykhuizen, and R. S. Ostfeld. 2008. Conspicuous impacts of inconspicuous hosts on the Lyme disease epidemic. Proc R Soc Biol Sci Ser B 275: 227-235.

Brossard, M., and S. K. Wikel. 2004. Tick immunobiology. Parasitology 129: S161-176.

Brunner, J. L., and R. S. Ostfeld. 2008. Multiple causes of variable tick burdens on smallmammal hosts. Ecology 89: 2259-2272.

Brunner, J. L., K. Logiudice, and R. S. Ostfeld. 2008. Estimating reservoir competence of Borrelia burgdorferi hosts: Prevalence and infectivity, sensitivity, and specificity. J. Med. Entomol. 45: 139-147.

Brunner, J. L., L. Cheney, F. Keesing, M. Killilea, K. Logiudice, A. Previtali, and R. S. Ostfeld. 2011. Molting success of Ixodes scapularis varies among individual blood meal hosts and species. J. Med. Entomol. 48: 860-866.

Bunnell, T., K. Hanisch, J. D. Hardege, and T. Breithaupt. 2011. The fecal odor of sick hedgehogs (Erinaceus europaeus) mediates olfactory attraction of the tick Ixodes hexagonus. J. Chem. Ecol. 37: 340-347.

Burgdorfer, W. 1984. Discovery of the Lyme disease spirochete and its relation to tick vectors. Yale J Biol Med 57: 515-520.

Burgdorfer, W., A. G. Barbour, S. F. Hayes, J. L. Benach, E. Grunwaldt, and J. P. Davis. 1982. Lyme disease - A tick-borne spirochetosis? Science 216: 1317-1319.

Burri, C., O. Schumann, C. Schumann, and L. Gern. 2014. Are Apodemus spp. mice and Myodes glareolus reservoirs for Borrelia miyamotoi, Candidatus Neoehrlichia mikurensis, Rickettsia helvetica, R. monacensis and Anaplasma phagocytophilum? Ticks Tick Borne Dis 5: 245-251.

Bursell, E. 1984. Observations on the orientation of tsetse flies (Glossina pallidipes) to windborne odours. Physiol Entomol 9: 133-137.

Cadenas, F. M., O. Rais, P. F. Humair, V. Douet, J. Moret, and L. Gern. 2007. Identification of host bloodmeal source and Borrelia burgdorferi sensu lato in field-collected Ixodes ricinus ticks in chaumont (Switzerland). J. Med. Entomol. 44: 1109-1117.

Callister, S. M., W. A. Agger, R. F. Schell, and K. M. Brand. 1989. Efficacy of the urinary bladder for isolation of Borrelia burgdorferi from naturally infected, wild Peromyscus leucopus. J. Clin. Microbiol. 27: 773-774. 
Carey, A. F., G. Wang, C. Y. Su, L. J. Zwiebel, and J. R. Carlson. 2010. Odorant reception in the malaria mosquito Anopheles gambiae. Nature 464: 66-71.

Carroll, J. F., J. A. Klun, and E. T. Schmidtmann. 1995. Evidence for kairomonal influence on selection of host-ambushing sites by adult Ixodes scapularis (Acari: Ixodidae). J. Med. Entomol. 32: 119-125.

Cator, L. J., J. George, S. Blanford, C. C. Murdock, T. C. Baker, A. F. Read, and M. B. Thomas. 2013. 'Manipulation' without the parasite: altered feeding behaviour of mosquitoes is not dependent on infection with malaria parasites. Proceedings. Biological sciences / The Royal Society 280: 20130711.

Chaisson, K. E., and E. A. Hallem. 2012. Chemosensory behaviors of parasites. Trends Parasitol 28: 427-436.

Clotfelter, E. D., A. B. Pedersen, J. A. Cranford, N. Ram, E. A. Snajdr, V. Nolan Jr, and E. D. Ketterson. 2007. Acorn mast drives long-term dynamics of rodent and songbird populations. Oecologia 154: 493-503.

Coipan, E. C., M. Fonville, E. Tijsse-Klasen, J. W. B. van der Giessen, W. Takken, H. Sprong, and K. Takumi. 2013a. Geodemographic analysis of Borrelia burgdorferi sensu lato using the 5S-23S rDNA spacer region. Infect., Genet. Evol. 17: 216-222.

Coipan, E. C., S. Jahfari, M. Fonville, C. B. Maassen, J. van der Giessen, W. Takken, K. Takumi, and H. Sprong. 2013b. Spatiotemporal dynamics of emerging pathogens in questing Ixodes ricinus. Front Cell Infect Microbiol 3: 36.

Coipan, E. C., S. Jahfari, M. Fonville, G. A. Oei, L. Spanjaard, K. Takumi, J. W. R. Hovius, and H. Sprong. 2016. Imbalanced presence of Borrelia burgdorferi s.l. multilocus sequence types in clinical manifestations of Lyme borreliosis. Infect., Genet. Evol. 42: 66-76.

Cook, M. J. 2015. Lyme borreliosis: A review of data on transmission time after tick attachment. Int J Gen Med 8: 1-8.

Cornet, S., A. Nicot, A. Rivero, and S. Gandon. 2013. Malaria infection increases bird attractiveness to uninfected mosquitoes. Ecol. Lett. 16: 323-329.

Cosson, J.-F., L. Michelet, J. Chotte, E. Le Naour, M. Cote, E. Devillers, M.-L. Poulle, D. Huet, M. Galan, J. Geller, S. Moutailler, and M. Vayssier-Taussat. 2014. Genetic characterization of the human relapsing fever spirochete Borrelia miyamotoi in vectors and animal reservoirs of Lyme disease spirochetes in France. Parasites \& Vectors 7: $1-5$.

Craine, N. G., S. E. Randolph, and P. A. Nuttall. 1995. Seasonal variation in the role of grey squirrels as hosts of Ixodes ricinus, the tick vector of the Lyme disease spirochaete, in a British woodland. Folia Parasitol. 42: 73-80. 
Crippa, M., O. Rais, and L. Gern. 2002. Investigations on the mode and dynamics of transmission and infectivity of Borrelia burgdorferi sensu stricto and Borrelia afzelii in Ixodes ricinus ticks. Vector Borne Zoonotic Dis. 2: 3-9.

Crooks, E., and S. E. Randolph. 2006. Walking by Ixodes ricinus ticks: Intrinsic and extrinsic factors determine the attraction of moisture or host odour. J. Exp. Biol. 209: 2138-2142.

Dallas, T., and S. Fore. 2013. Chemical attraction of Dermacentor variabilis ticks parasitic to Peromyscus leucopus based on host body mass and sex. Exp. Appl. Acarol. 61: 243250.

Daniel, M., M. Malý, V. Danielová, B. Kəíž, and P. Nuttall. 2015. Abiotic predictors and annual seasonal dynamics of Ixodes ricinus, the major disease vector of Central Europe. Parasit Vectors 8.

Dantas-Torres, F., B. B. Chomel, and D. Otranto. 2012. Ticks and tick-borne diseases: A One Health perspective. Trends Parasitol 28: 437-446.

de Boer, R., K. E. Hovius, M. K. Nohlmans, and J. S. Gray. 1993a. The woodmouse (Apodemus sylvaticus) as a reservoir of tick-transmitted spirochetes (Borrelia burgdorferi) in The Netherlands. Zentralbl Bakteriol 279: 404-416.

De Boer, R., K. E. Hovius, M. K. Nohlmans, and J. S. Gray. 1993b. The woodmouse (Apodemus sylvaticus) as a reservoir of tick-transmitted spirochetes (Borrelia burgdorferi) in The Netherlands. Zentralbl Bakteriol 279: 404-416.

De Moraes, C. M., N. M. Stanczyk, H. S. Betz, H. Pulido, D. G. Sim, A. F. Read, and M. C. Mescher. 2014. Malaria-induced changes in host odors enhance mosquito attraction. Proc Natl Acad Sci U S A 111: 11079-11084.

Demas, G. E., V. Chefer, M. I. Talan, and R. J. Nelson. 1997. Metabolic costs of mounting an antigen-stimulated immune response in adult and aged C57BL/6J mice. American Journal of Physiology - Regulatory Integrative and Comparative Physiology 273: R1631-R1637.

Dennison, N. J., N. Jupatanakul, and G. Dimopoulos. 2014. The mosquito microbiota influences vector competence for human pathogens. Curr Opin Insect Sci 3: 6-13.

Devevey, G., and D. Brisson. 2012. The effect of spatial heterogenity on the aggregation of ticks on white-footed mice. Parasitology 139: 915-925.

Díaz, M., and C. L. Alonso. 2003. Wood mouse Apodemus sylvaticus winter food supply: Density, condition, breeding, and parasites. Ecology 84: 2680-2691.

Diuk-Wasser, M. A., A. G. Hoen, P. Cislo, R. Brinkerhoff, S. A. Hamer, M. Rowland, R. Cortinas, G. Vourc'h, F. Melton, G. J. Hickling, J. I. Tsao, J. Bunikis, A. G. Barbour, U. Kitron, J. Piesman, and D. Fish. 2012. Human risk of infection with Borrelia burgdorferi, the lyme disease agent, in Eastern United States. Am. J. Trop. Med. Hyg. 86: 320-327. 
Dizij, A., and K. Kurtenbach. 1995. Clethrionomys glareolus, but not Apodemus flavicollis acquires resistance to Ixodes ricinus L., the main European vector of Borrelia burgdorferi. Parasite Immunol. 17: 177-183.

Dunham-Ems, S. M., M. J. Caimano, U. Pal, C. W. Wolgemuth, C. H. Eggers, A. Balic, and J. D. Radolf. 2009. Live imaging reveals a biphasic mode of dissemination of Borrelia burgdorferi within ticks. J. Clin. Invest. 119: 3652-3665.

Estrada-Pena, A., C. Ortega, N. Sanchez, L. Desimone, B. Sudre, J. E. Suk, and J. C. Semenza. 2011. Correlation of Borrelia burgdorferi sensu lato prevalence in questing Ixodes ricinus ticks with specific abiotic traits in the western palearctic. Appl. Environ. Microbiol. 77: 3838-3845.

Estrada-Peña, A., J. S. Gray, O. Kahl, R. S. Lane, and A. M. Nijhof. 2013. Research on the ecology of ticks and tick-borne pathogens--methodological principles and caveats. Front Cell Infect Microbiol 3: 29.

Faulde, M. K., and R. G. Robbins. 2008. Tick infestation risk and Borrelia burgdorferi s.I. infection-induced increase in host-finding efficacy of female Ixodes ricinus under natural conditions. Exp. Appl. Acarol. 44: 137-145.

Fingerle, V., U. C. Schulte-Spechtel, E. Ruzic-Sabljic, S. Leonhard, H. Hofmann, K. Weber, K. Pfister, F. Strle, and B. Wilske. 2008. Epidemiological aspects and molecular characterization of Borrelia burgdorferi s.I. from southern Germany with special respect to the new species Borrelia spielmanii sp. nov. Int. J. Med. Microbiol. 298: 279-290.

Fonville, M., P. Hengeveld, A. Docters van Leeuwen, S. Jahfari, M. G. Harms, A. J. H. van Vliet, W. van Pelt, H. Sprong, and C. C. van den Wijngaard. 2013. Blootstelling aan Borrelia miyamotoi door tekenbeten Infectieziekten Bulletin 24: 4.

Garcia, R. 1962. Carbon dioxide as an attractant for certain ticks (Acarina: Argasidae and Ixodidae). Ann. Entomol. Soc. Am. 55: 605-606.

Gassner, F. 2010. Tick Tactics - Interactions between habitat characteristics, hosts and microorganisms in relation to the biology of the sheep tick Ixodes ricinus. PhD, Wageningen University Wageningen.

Gassner, F., P. Verbaarschot, R. C. Smallegange, J. Spitzen, S. E. Van Wieren, and W. Takken. 2008. Variations in Ixodes ricinus density and Borrelia infections associated with cattle introduced into a woodland in The Netherlands. Appl. Environ. Microbiol. 74: 7138-7144.

Gassner, F., W. Takken, C. L. Plas, P. Kastelein, A. J. Hoetmer, M. Holdinga, and L. S. van Overbeek. 2013. Rodent species as natural reservoirs of Borrelia burgdorferi sensu lato in different habitats of Ixodes ricinus in The Netherlands. Ticks Tick Borne Dis 4: $452-458$. 
Gassner, F., A. J. van Vliet, S. L. Burgers, F. Jacobs, P. Verbaarschot, E. K. Hovius, S. Mulder, N. O. Verhulst, L. S. van Overbeek, and W. Takken. 2011. Geographic and temporal variations in population dynamics of Ixodes ricinus and associated Borrelia infections in The Netherlands. Vector Borne Zoonotic Dis. 11: 523-532.

Gern, L., and O. Rais. 1996. Efficient transmission of Borrelia burgdorferi between cofeeding Ixodes ricinus ticks (Acari: Ixodidae). J. Med. Entomol. 33: 189-192.

Gern, L., N. Lebet, and J. Moret. 1996. Dynamics of Borrelia burgdorferi infection in nymphal Ixodes ricinus ticks during feeding. Exp. Appl. Acarol. 20: 649-658.

Gern, L., M. Siegenthaler, C. M. Hu, S. Leuba-Garcia, P. F. Humair, and J. Moret. 1994. Borrelia burgdorferi in rodents (Apodemus flavicollis and A. sylvaticus): Duration and enhancement of infectivity for Ixodes ricinus ticks. Eur. J. Epidemiol. 10: 75-80.

Gern, L. Z., L.; Aeschlimann, A. . 1990. Development of Borrelia burgdorferi in Ixodes ricinus females during blood feeding. Ann Parasitol Hum Comp 62: 5.

Gherman, C. M., A. D. Mihalca, M. O. Dumitrache, A. Györke, I. Oroian, M. Sandor, and V. Cozma. 2012. $\mathrm{CO}_{2}$ flagging - An improved method for the collection of questing ticks. Parasit Vectors 5: 125.

Gillespie, R. D., M. L. Mbow, and R. G. Titus. 2000. The immunomodulatory factors of bloodfeeding arthropod saliva. Parasite Immunol. 22: 319-331.

Gray, J. S. 1985. A carbon dioxide trap for prolonged sampling of Ixodes ricinus L. populations. Experimental \& Applied Acarology 1: 35-44.

Gray, J. S. 1998. The ecology of ticks transmitting Lyme borreliosis. Exp. Appl. Acarol. 22: 249-258.

Gray, J. S., O. Kahl, C. Janetzki, and J. Stein. 1992. Studies on the ecology of Lyme disease in a deer forest in County Galway, Ireland. J. Med. Entomol. 29: 915-920.

Gray, J. S., F. Kirstein, J. N. Robertson, J. Stein, and O. Kahl. 1999. Borrelia burgdorferi sensu lato in Ixodes ricinus ticks and rodents in a recreational park in south-western Ireland. Exp. Appl. Acarol. 23: 717-729.

Grubhoffer, L., M. Golovchenko, M. Vancová, K. Zacharovová-Slavíčková, N. Rudenko, and J. H. Oliver Jr. 2005. Lyme borreliosis: Insights into tick-/host-Borrelia relations. Folia Parasitol. 52: 279-294.

Guerenstein, P. G., and P. M. Guerin. 2001. Olfactory and behavioural responses of the blood-sucking bug Triatoma infestans to odours of vertebrate hosts. J. Exp. Biol. 204: 585-597.

Hafer, N., and M. Milinski. 2015. When parasites disagree: Evidence for parasite-induced sabotage of host manipulation. Evolution 69: 611-620. 
Hajdusek, O., R. Sima, N. Ayllon, M. Jalovecka, J. Perner, J. de la Fuente, and P. Kopacek. 2013. Interaction of the tick immune system with transmitted pathogens. Front Cell Infect Microbiol 3: 26.

Hammer, B., T. Meiners, A. Marangoni, V. Sambri, U. B. Göbel, and O. Kahl. 2002. Borrelia burgdorferi sensu lato transmission from tick vector to host: Experimental evidence of the involvement of the larval stage of Ixodes ricinus. Int. J. Med. Microbiol. 291, Supplement 33: 211.

Hanincová, K., S. M. Schäfer, S. Etti, H. S. Sewell, V. Taragelová, D. Ziak, M. Labuda, and K. Kurtenbach. 2003a. Association of Borrelia afzelii with rodents in Europe. Parasitology 126: 11-20.

Hanincová, K., V. Taragelová, J. Koci, S. M. Schäfer, R. Hails, A. J. Ullmann, J. Piesman, M. Labuda, and K. Kurtenbach. 2003b. Association of Borrelia garinii and $B$. valaisiana with songbirds in Slovakia. Appl. Environ. Microbiol. 69: 2825-2830.

Harris, P. D., A. Paziewska, L. Zwolińska, and E. Siński. 2009. Seasonality of the ectoparasite community of woodland rodents in a Mazurian Forest, Poland. Wiad Parazytol 55: 377-388.

Harrison, A., and N. C. Bennett. 2012. The importance of the aggregation of ticks on small mammal hosts for the establishment and persistence of tick-borne pathogens: An investigation using the R 0 model. Parasitology 139: 1605-1613.

Harrison, A., M. Scantlebury, and W. I. Montgomery. 2010. Body mass and sex-biased parasitism in wood mice Apodemus sylvaticus. Oikos 119: 1099-1104.

Hellgren, O., M. Andersson, and L. Råberg. 2011. The genetic structure of Borrelia afzelii varies with geographic but not ecological sampling scale. J. Evol. Biol. 24: 159-167.

Herrmann, C., and L. Gern. 2010. Survival of Ixodes ricinus (Acari: Ixodidae) under challenging conditions of temperature and humidity is influenced by Borrelia burgdorferi sensu lato infection. J. Med. Entomol. 47: 1196-1204.

Herrmann, C., and L. Gern. 2012. Do the level of energy reserves, hydration status and Borrelia infection influence walking by Ixodes ricinus (Acari: Ixodidae) ticks? Parasitology 139: 330-337.

Herrmann, C., and L. Gern. 2015. Search for blood or water is influenced by Borrelia burgdorferi in Ixodes ricinus. Parasit Vectors 8: 6.

Herrmann, C., L. Gern, and M. J. Voordouw. 2013a. Species co-occurrence patterns among lyme borreliosis pathogens in the tick vector Ixodes ricinus. Appl. Environ. Microbiol. 79: 7273-7280.

Herrmann, C., M. J. Voordouw, and L. Gern. 2013b. Ixodes ricinus ticks infected with the causative agent of Lyme disease, Borrelia burgdorferi sensu lato, have higher energy reserves. Int. J. Parasitol. 43: 477-483. 
Heylen, D., E. Matthysen, M. Fonville, and H. Sprong. 2013a. Songbirds as general transmitters but selective amplifiers of Borrelia burgdorferi sensu lato genotypes in Ixodes ricinus ticks. Environ. Microbiol. 16: 2859-2868.

Heylen, D., M. Fonville, A. D. van Leeuwen, and H. Sprong. 2016. Co-infections and transmission dynamics in a tick-borne bacterium community exposed to songbirds. Environ. Microbiol. 18: 988-996.

Heylen, D., E. Tijsse, M. Fonville, E. Matthysen, and H. Sprong. 2013b. Transmission dynamics of Borrelia burgdorferi s.I. in a bird tick community. Environ. Microbiol. 15: 663-673.

Heylen, D., F. Adriaensen, S. Van Dongen, H. Sprong, and E. Matthysen. 2013c. Ecological factors that determine Ixodes ricinus tick burdens in the great tit (Parus major), an avian reservoir of Borrelia burgdorferi s.I. Int. J. Parasitol. 43: 603-611.

Heylen, D., H. Sprong, K. van Oers, M. Fonville, H. Leirs, and E. Matthysen. 2014. Are the specialized bird ticks, Ixodes arboricola and I. frontalis, competent vectors for Borrelia burgdorferi sensu lato? Environ. Microbiol. 16: 1081-1089.

Heylen, D. J. A., and E. Matthysen. 2011. Experimental evidence for host preference in a tick parasitizing songbird nestlings. Oikos 120: 1209-1216.

Hofhuis, A., M. Harms, C. van den Wijngaard, H. Sprong, and W. van Pelt. 2015. Continuing increase of tick bites and Lyme disease between 1994 and 2009. Ticks Tick Borne Dis 6: 69-74.

Hofhuis, A., T. Herremans, D. W. Notermans, H. Sprong, M. Fonville, J. W. van der Giessen, and W. van Pelt. 2013. A prospective study among patients presenting at the general practitioner with a tick bite or erythema migrans in The Netherlands. PLoS One 8: e64361.

Hofhuis, A. H., M.G.; van der Giessen, J.W.B.; Sprong, H.; Notermans, D.W.; van Pelt, W. 2010. Ziekte van Lyme in Nederland 1994 - 2009: aantal huisartsconsulten blijft toenemen. Is voorlichting en curatief beleid genoeg? Infectieziekten Bulletin 21: 84-87.

Hofmeester, T. R., E. C. Coipan, S. E. Van Wieren, H. H. T. Prins, W. Takken, and H. Sprong. 2016. Few vertebrate species dominate the Borrelia burgdorferi s.I. Life cycle. Environmental Research Letters 11.

Hofmeister, E. K., R. B. Markham, J. E. Childs, and R. R. Arthur. 1992. Comparison of polymerase chain-reaction and culture for detection of Borrelia burgdorferi in naturally infected Peromyscus leucopus and experimentally infected C.B-17 scid scid mice. J. Clin. Microbiol. 30: 2625-2631.

Hofmeister, E. K., B. A. Ellis, G. E. Glass, and J. E. Childs. 1999. Longitudinal study of infection with Borrelia burgdorferi in a population of Peromyscus leucopus at a Lyme disease-enzootic site in Maryland. Am. J. Trop. Med. Hyg. 60: 598-609. 
Hovius, J. W., X. Li, N. Ramamoorthi, A. P. van Dam, S. W. Barthold, T. van der Poll, P. Speelman, and E. Fikrig. 2007. Coinfection with Borrelia burgdorferi sensu stricto and Borrelia garinii alters the course of murine Lyme borreliosis. FEMS Immunol. Med. Microbiol. 49: 224-234.

Hovius, J. W., B. de Wever, M. Sohne, M. C. Brouwer, J. Coumou, A. Wagemakers, A. Oei, H. Knol, S. Narasimhan, C. J. Hodiamont, S. Jahfari, S. T. Pals, H. M. Horlings, E. Fikrig, H. Sprong, and M. H. van Oers. 2013. A case of meningoencephalitis by the relapsing fever spirochaete Borrelia miyamotoi in Europe. Lancet 382: 658.

Hu, C. M., P. F. Humair, R. Wallich, and L. Gern. 1997a. Apodemus sp. rodents, reservoir hosts for Borrelia afzelii in an endemic area in Switzerland. Zentralbl Bakteriol 285: 558-564.

Hu, R., K. E. Hyland, and D. Markowski. 1997b. Effects of Babesia microti infection on feeding pattern, engorged body weight, and molting rate of immature Ixodes scapularis (Acari: Ixodidae). J. Med. Entomol. 34: 559-564.

Hubalek, Z., J. Halouzka, Z. Juricova, and S. Svobodova. 1994. Seasonal distribution of Borreliae in Ixodes ricinus ticks. Zentralbl Bakteriol 280: 423-431.

Huegli, D., C. M. Hu, P. F. Humair, B. Wilske, and L. Gern. 2002. Apodemus species mice are reservoir hosts of Borrelia garinii OspA serotype 4 in Switzerland. J. Clin. Microbiol. 40: 4735-4737.

Hughes, V. L., and S. E. Randolph. 2001. Testosterone depresses innate and acquired resistance to ticks in natural rodent hosts: a force for aggregated distributions of parasites. J. Parasitol. 87: 49-54.

Humair, P. F., O. Rais, and L. Gern. 1999. Transmission of Borrelia afzelii from Apodemus mice and Clethrionomys voles to Ixodes ricinus ticks: differential transmission pattern and overwintering maintenance. Parasitology 118: 33-42.

Humair, P. F., N. Turrian, A. Aeschlimann, and L. Gern. 1993. Borrelia burgdorferi in a focus of Lyme borreliosis - Epizootiologic contribution of small mammals. Folia Parasitol. 40: 65-70.

Humair, P. F., O. Peter, R. Wallich, and L. Gern. 1995. Strain variation of Lyme disease spirochetes isolated from Ixodes ricinus ticks and rodents collected in two endemic areas in Switzerland. J. Med. Entomol. 32: 433-438.

Hurd, H. 2003. Manipulation of medically important insect vectors by their parasites. Annu. Rev. Entomol. 48: 141-161.

Jacquot, M., M. Bisseux, D. Abrial, M. Marsot, E. Ferquel, J. L. Chapuis, G. Vourc'h, and X. Bailly. 2014. High-throughput sequence typing reveals genetic differentiation and host specialization among populations of the Borrelia burgdorferi species complex that infect rodents. PLoS ONE 9: e88581. 
Jaenson, T. G. T., and L. Talleklint. 1992. Incompetence of roe deer as reservoirs of the Lyme borreliosis spirochete. J. Med. Entomol. 29: 813-817.

Jahfari, S., E. C. Coipan, M. Fonville, A. D. van Leeuwen, P. Hengeveld, D. Heylen, P. Heyman, C. van Maanen, C. M. Butler, G. Foldvari, S. Szekeres, G. van Duijvendijk, W. Tack, J. M. Rijks, J. van der Giessen, W. Takken, S. E. van Wieren, K. Takumi, and H. Sprong. 2014. Circulation of four Anaplasma phagocytophilum ecotypes in Europe. Parasit Vectors 7: 365.

James, A. M., and J. H. Oliver Jr. 1990. Feeding and host preference of immature Ixodes dammini, I. scapularis, and I. pacificus (Acari: Ixodidae). J. Med. Entomol. 27: 324-330.

Jensen, T. S. 1982. Seed production and outbreaks of non-cyclic rodent populations in deciduous forests. Oecologia 54: 184-192.

Johns, R., J. Ohnishi, A. Broadwater, D. E. Sonenshine, A. M. De Silva, and W. L. Hynes. 2001. Contrasts in tick innate immune responses to Borrelia burgdorferi challenge: immunotolerance in Ixodes scapularis versus immunocompetence in Dermacentor variabilis (Acari: Ixodidae). J. Med. Entomol. 38: 99-107.

Jones, C., J. Brunner, G. Scoles, and J. P. Owen. 2015. Factors affecting larval tick feeding success: host, density and time. Parasit Vectors 8: 340.

Jones, C. G., R. S. Ostfeld, M. P. Richard, E. M. Schauber, and J. O. Wolff. 1998. Chain reactions linking acorns to gypsy moth outbreaks and Lyme disease risk. Science 279: 1023-1026.

Kahl, O., C. Janetzki-Mittmann, J. S. Gray, R. Jonas, J. Stein, and R. De Boer. 1998. Risk of infection with Borrelia burgdorferi sensu lato for a host in relation to the duration of nymphal Ixodes ricinus feeding and the method of tick removal. Zentralbl Bakteriol 287: 41-52.

Kaufman, W. R. 1989. Tick-host interaction - a synthesis of current concepts. Parasitol Today 5: 47-56.

Kavaliers, M., and D. D. Colwell. 1995a. Discrimination by female mice between the odours of parasitized and non-parasitized males. Proc R Soc Biol Sci Ser B 261: 31-35.

Kavaliers, M., and D. D. Colwell. 1995b. Odours of parasitized males induce aversive responses in female mice. Anim. Behav. 50: 1161-1169.

Keesing, F., J. Brunner, S. Duerr, M. Killilea, K. Logiudice, K. Schmidt, H. Vuong, and R. S. Ostfeld. 2009. Hosts as ecological traps for the vector of Lyme disease. Proc R Soc Biol Sci Ser B 276: 3911-3919.

Keirans, J. E., H. J. Hutcheson, L. A. Durden, and J. S. H. Klompen. 1996. Ixodes (Ixodes) scapularis (Acari: Ixodidae): Redescription of all Active Stages, Distribution, Hosts, Geographical Variation, and Medical and Veterinary Importance. J. Med. Entomol. 33: 297-318. 
Kelly, D., and V. L. Sork. 2002. Mast seeding in perennial plants: Why, how, where? Annu. Rev. Ecol. Syst. 33: 427-447.

Kempf, F., T. De Meeus, E. Vaumourin, V. Noel, V. Taragel'ova, O. Plantard, D. J. A. Heylen, C. Eraud, C. Chevillon, and K. D. McCoy. 2011. Host races in Ixodes ricinus, the European vector of Lyme borreliosis. Infect Genet Evol 11: 2043-2048.

Khanakah, G., E. Kocianova, V. Vyrostekova, J. Rehacek, M. Kundi, and G. Stanek. 2006. Seasonal variations in detecting Borrelia burgdorferi sensu lato in rodents from north eastern Austria. Wien Klin Wochenschr 118: 754-758.

Khokhlova, I. S., B. R. Krasnov, M. Kam, N. I. Burdelova, and A. A. Degen. 2002. Energy cost of ectoparasitism: the flea Xenopsylla ramesis on the desert gerbil Gerbillus dasyurus. J. Zool. 258: 349-354.

Kiewra, D., J. Stańczak, and M. Richter. 2014. Ixodes ricinus ticks (Acari, Ixodidae) as a vector of Borrelia burgdorferi sensu lato and Borrelia miyamotoi in Lower Silesia, Poland - Preliminary study. Ticks Tick Borne Dis 5: 892-897.

Kiffner, C., T. Vor, P. Hagedorn, M. Niedrig, and F. Rühe. 2011. Factors affecting patterns of tick parasitism on forest rodents in tick-borne encephalitis risk areas, Germany. Parasitol Res 108: 323-335.

Killick Kendrick, R., A. J. Leaney, P. D. Ready, and D. H. Molyneux. 1977. Leishmania in phlebotomid sandflies. IV. The transmission of Leishmania mexicana amazonensis to hamsters by the bite of experimentally infected Lutzomyia lonigpalpis. Proceedings of the Royal Society of London - Biological Sciences 196: 105-115.

Kjelland, V., S. Stuen, T. Skarpaas, and A. Slettan. 2010. Prevalence and genotypes of Borrelia burgdorferi sensu lato infection in Ixodes ricinus ticks in southern Norway. Scand J Infect Dis 42: 579-585.

Kjelland, V., R. Rollum, L. Korslund, A. Slettan, and D. Tveitnes. 2015. Borrelia miyamotoi is widespread in Ixodes ricinus ticks in southern Norway. Ticks Tick Borne Dis 6: 516521.

Klein, S. L., H. R. Gamble, and R. J. Nelson. 1999. Trichinella spiralis infection in voles alters female odor preference but not partner preference. Behav. Ecol. Sociobiol. 45: 323329.

Koella, J. C., F. L. Sorensen, and R. A. Anderson. 1998. The malaria parasite, Plasmodium falciparum, increases the frequency of multiple feeding of its mosquito vector, Anopheles gambiae. Proc R Soc Biol Sci Ser B 265: 763-768.

Koenraadt, C. J. M., and M. Dicke. 2010. The role of volatiles in aggregation and host-seeking of the haematophagous poultry red mite Dermanyssus gallinae (Acari: Dermanyssidae). Exp. Appl. Acarol. 50: 191-199. 
Krasnov, B. R., M. Stanko, and S. Morand. 2007. Host community structure and infestation by ixodid ticks: Repeatability, dilution effect and ecological specialization. Oecologia 154: 185-194.

Krause, P. J., D. Fish, S. Narasimhan, and A. G. Barbour. 2015. Borrelia miyamotoi infection in nature and in humans. Clin. Microbiol. Infect. 21: 631-639.

Krebs, C. J. 2013. Population Fluctuations in Rodents, University of Chicago Press, Chicago.

Kurtenbach, K., H. S. Sewell, N. H. Ogden, S. E. Randolph, and P. A. Nuttall. 1998a. Serum complement sensitivity as a key factor in Lyme disease ecology. Infect. Immun. 66: 1248-1251.

Kurtenbach, K., M. Peacey, S. G. Rijpkema, A. N. Hoodless, P. A. Nuttall, and S. E. Randolph. 1998b. Differential transmission of the genospecies of Borrelia burgdorferi sensu lato by game birds and small rodents in England. Appl. Environ. Microbiol. 64: 1169-1174.

Kurtenbach, K., H. Kampen, A. Dizij, S. Arndt, H. M. Seitz, U. E. Schaible, and M. M. Simon. 1995. Infestation of rodents with larval Ixodes ricinus (Acari: Ixodidae) is an important factor in the transmission cycle of Borrelia burgdorferi s.l. in German woodlands. J. Med. Entomol. 32: 807-817.

Kurtenbach, K., S. De Michelis, S. Etti, S. M. Schäfer, H. S. Sewell, V. Brade, and P. Kraiczy. 2002a. Host association of Borrelia burgdorferi sensu lato - The key role of host complement. Trends Microbiol 10: 74-79.

Kurtenbach, K., S. M. Schäfer, H. S. Sewell, M. Peacey, A. Hoodless, P. A. Nuttall, and S. E. Randolph. 2002b. Differential survival of lyme borreliosis spirochetes in ticks that feed on birds. Infect. Immun. 70: 5893-5895.

Kurtenbach, K., A. Dizij, H. M. Seitz, G. Margos, S. E. Moter, M. D. Kramer, R. Wallich, U. E. Schaible, and M. M. Simon. 1994. Differential immune-responses to Borrelia burgdorferi in European wild rodent species influence spirochete transmission to Ixodes ricinus L (Acari, Ixodidae). Infect. Immun. 62: 5344-5352.

Kurtenbach, K., S. De Michelis, H. S. Sewell, S. Etti, S. M. Schafer, E. Holmes, R. Hails, M. Collares-Pereira, M. Santos-Reis, K. Hanincová, M. Labuda, A. Bormane, and M. Donaghy. 2002c. The key roles of selection and migration in the ecology of Lyme borreliosis. Int. J. Med. Microbiol. 291 Suppl 33: 152-154.

Kybicova, K., Z. Kurzova, and D. Hulinska. 2008. Molecular and serological evidence of Borrelia burgdorferi sensu lato in wild rodents in the Czech Republic. Vector Borne Zoonotic Dis. 8: 645-652.

Lacroix, R., W. R. Mukabana, L. C. Gouagna, and J. C. Koella. 2005. Malaria infection increases attractiveness of humans to mosquitoes. PLoS Biol. 3: 1590-1593. 
Lees, A. D. 1946. The water balance in Ixodes ricinus L. and certain other species of ticks. Parasitology 37: 1-20.

Lees, A. D. 1948. The sensory physiology of the sheep tick, Ixodes ricinus L. J. Exp. Biol. 25: 145-207.

Lefcort, H., and L. A. Durden. 1996. The effect of infection with Lyme disease spirochetes (Borrelia burgdorferi) on the phototaxis, activity, and questing height of the tick vector Ixodes scapularis. Parasitology 113: 97-103.

Lefèvre, T., and F. Thomas. 2008. Behind the scene, something else is pulling the strings: Emphasizing parasitic manipulation in vector-borne diseases. Infect., Genet. Evol. 8: 504-519.

Leuba-Garcia, S., M. D. Kramer, R. Wallich, and L. Gern. 1994. Characterization of Borrelia burgdorferi isolated from different organs of Ixodes ricinus ticks collected in nature. Zentralbl Bakteriol 280: 468-475.

Levi, T., A. M. Kilpatrick, M. Mangel, and C. C. Wilmers. 2012. Deer, predators, and the emergence of Lyme disease. Proc Natl Acad Sci U S A 109: 10942-10947.

Levin, M. L., and D. Fish. 1998. Density-dependent factors regulating feeding success of Ixodes scapularis larvae (Acari : Ixodidae). J. Parasitol. 84: 36-43.

Li, S., P. Heyman, C. Cochez, L. Simons, and S. O. Vanwambeke. 2012. A multi-level analysis of the relationship between environmental factors and questing Ixodes ricinus dynamics in Belgium. Parasit Vectors 5.

Lindblom, P., P. Wilhelmsson, L. Fryland, J. Sjöwall, M. Haglund, A. Matussek, J. Ernerudh, S. Vene, D. Nyman, T. Andreassen, P. Forsberg, and P. E. Lindgren. 2014. Tick-borne encephalitis virus in ticks detached from humans and follow-up of serological and clinical response. Ticks Tick Borne Dis 5: 21-28.

Lindsay, L. R., I. K. Barker, G. A. Surgeoner, S. A. McEwen, and G. D. Campbell. 1997. Duration of Borrelia burgdorferi infectivity in white-footed mice for the tick vector Ixodes scapularis under laboratory and field conditions in Ontario. J. Wildl. Dis. 33: 766-775.

Liu, X. Y., M. Cote, R. E. Paul, and S. I. Bonnet. 2014. Impact of feeding system and infection status of the blood meal on Ixodes ricinus feeding. Ticks Tick Borne Dis 5: 323-328.

Lloyd-Smith, J. O., S. J. Schreiber, P. E. Kopp, and W. M. Getz. 2005. Superspreading and the effect of individual variation on disease emergence. Nature 438: 355-359.

LoGiudice, K., R. S. Ostfeld, K. A. Schmidt, and F. Keesing. 2003. The ecology of infectious disease: Effects of host diversity and community composition on lyme disease risk. Proc Natl Acad Sci U S A 100: 567-571.

Mallorie, H. C., and J. R. Flowerdew. 1994. Woodland small mammal population ecology in Britain: A preliminary review of the Mammal Society survey of wood mice Apodemus sylvaticus and bank voles Clethrionomys glareolus, 1982-87. MAMMAL REV. 24: 1-15. 
Mannelli, A., L. Bertolotti, L. Gern, and J. Gray. 2012. Ecology of Borrelia burgdorferi sensu lato in Europe: transmission dynamics in multi-host systems, influence of molecular processes and effects of climate change. FEMS Microbiol Rev 36: 837-861.

Mappes, T. 1998. High population density in bank voles stimulates food hoarding after breeding. Anim. Behav. 55: 1483-1487.

Margos, G., S. A. Vollmer, M. Cornet, M. Garnier, V. Fingerle, B. Wilske, A. Bormane, L. Vitorino, M. Collares-Pereira, M. Drancourt, and K. Kurtenbach. 2009. A new Borrelia species defined by multilocus sequence analysis of housekeeping genes. Appl. Environ. Microbiol. 75: 5410-5416.

Marsot, M., J. L. Chapuis, P. Gasqui, A. Dozieres, S. Masseglia, B. Pisanu, E. Ferquel, and G. Vourc'h. 2013. Introduced Siberian chipmunks (Tamias sibiricus barberi) contribute more to lyme borreliosis risk than native reservoir rodents. PLoS One 8: e55377.

Mather, T. N., S. R. Telford, and G. H. Adler. 1991. Absence of transplacental transmission of Lyme disease spirochetes from reservoir mice (Peromyscus leucopus) to their offspring. J. Infect. Dis. 164: 564-567.

Matuschka, F. R., and A. Spielman. 1992. Loss of Lyme disease spirochetes from Ixodes ricinus ticks feeding on European blackbirds. Exp. Parasitol. 74: 151-158.

Matuschka, F. R., P. Fischer, K. Musgrave, D. Richter, and A. Spielman. 1991. Hosts on which nymphal Ixodes ricinus most abundantly feed. Am. J. Trop. Med. Hyg. 44: 100107.

Matuschka, F. R., P. Fischer, M. Heiler, D. Richter, and A. Spielman. 1992a. Capacity of European animals as reservoir hosts for the Lyme disease spirochete. J. Infect. Dis. 165: 479-483.

Matuschka, F. R., P. Fischer, M. Heiler, S. Blumcke, and A. Spielman. 1992b. Stageassociated risk of transmission of the Lyme disease spirochete by European Ixodes ticks. Parasitol Res 78: 695-698.

McCoy, K. D., E. Léger, and M. Dietrich. 2013. Host specialization in ticks and transmission of tick-borne diseases: a review. Frontiers in cellular and infection microbiology 3: 57.

McShea, W. J. 2000. The influence of acorn crops on annual variation in rodent and bird populations. Ecology 81: 228-238.

Medlock, J. M., K. M. Hansford, A. Bormane, M. Derdakova, A. Estrada-Pena, J. C. George, I. Golovljova, T. G. Jaenson, J. K. Jensen, P. M. Jensen, M. Kazimirova, J. A. Oteo, A. Papa, K. Pfister, O. Plantard, S. E. Randolph, A. Rizzoli, M. M. Santos-Silva, H. Sprong, L. Vial, G. Hendrickx, H. Zeller, and W. Van Bortel. 2013. Driving forces for changes in geographical distribution of Ixodes ricinus ticks in Europe. Parasit Vectors 6: 1. 
Mejlon, H. A., and T. G. T. Jaenson. 1997. Questing behaviour of Ixodes ricinus ticks (Acari: Ixodidae). Exp. Appl. Acarol. 21: 747-754.

Michalik, J., B. Skotarczak, M. Skoracki, B. Wodecka, B. Sikora, T. Hofman, A. Rymaszewska, and M. Sawczuk. 2005. Borrelia burgdorferi sensu stricto in yellownecked mice and feeding Ixodes ricinus ticks in a forest habitat of west central Poland. J. Med. Entomol. 42: 850-856.

Milne, A. 1943. The comparison of sheep-tick populations (Ixodes ricinus L.). Ann. Appl. Biol. 30: $240-250$.

Minchella, D. J. 1985. Host life-history variation in response to parasitism. Parasitology 90: 205-216.

Misonne, M. C., G. Van Impe, and P. P. Hoet. 1998. Genetic heterogeneity of Borrelia burgdorferi sensu lato in Ixodes ricinus ticks collected in Belgium. J. Clin. Microbiol. 36: 3352-3354.

Molloy, P. J., S. R. Telford, III, H. R. Chowdri, T. J. Lepore, J. L. Gugliotta, K. E. Weeks, M. E. Hewins, H. K. Goethert, and V. P. Berardi. 2015. Borrelia miyamotoi disease in the northeastern United States a case series. Ann Intern Med 163: 91-98.

Molyneux, D. H., and D. Jefferies. 1986. Feeding behaviour of pathogen-infected vectors. Parasitology 92 ( Pt 3): 721-736.

Montgomery, W. I. 1989. Population regulation in the wood mouse, Apodemus sylvaticus. I. Density dependence in the annual cycle of abundance. J. Anim. Ecol. 58: 465-475.

Moore, J. 1995. The behavior of parasitized animals. Bioscience 45: 89-96.

Morán-Cadenas, F. M., O. Rais, P. F. Humair, V. Douet, J. Moret, and L. Gern. 2007. Identification of host bloodmeal source and Borrelia burgdorferi sensu lato in fieldcollected Ixodes ricinus ticks in chaumont (Switzerland). J. Med. Entomol. 44: 11091117.

Moshkin, M., L. Gerlinskaya, O. Morozova, V. Bakhvalova, and V. Evsikov. 2002. Behaviour, chemosignals and endocrine functions in male mice infected with tick-borne encephalitis virus. Psychoneuroendocrinology 27: 603-608.

Murray, D. L., L. B. Keith, and J. R. Cary. 1998. Do parasitism and nutritional status interact to affect production in snowshoe hares? Ecology 79: 1209-1222.

Mysterud, A., I. L. Hatlegjerde, and O. J. Sørensen. 2014. Attachment site selection of life stages of Ixodes ricinus ticks on a main large host in Europe, the red deer (Cervus elaphus). Parasites \&amp; vectors 7: 510.

Mysterud, A., R. Byrkjeland, L. Qviller, and H. Viljugrein. 2015. The generalist tick Ixodes ricinus and the specialist tick Ixodes trianguliceps on shrews and rodents in a northern forest ecosystem- a role of body size even among small hosts. Parasit Vectors 8.

Narasimhan, S., and E. Fikrig. 2015. Tick microbiome: The force within. Trends Parasitol. 
Narasimhan, S., N. Rajeevan, L. Liu, Y. O. Zhao, J. Heisig, J. Pan, R. Eppler-Epstein, K. Deponte, D. Fish, and E. Fikrig. 2014. Gut microbiota of the tick vector Ixodes scapularis modulate colonization of the Lyme disease spirochete. Cell Host Microbe 15: 58-71.

Nau, R., H. J. Christen, and H. Eiffert. 2009. Lyme disease--current state of knowledge. Dtsch Arztebl Int 106: 72-81.

Nazario, S., S. Das, A. M. de Silva, K. Deponte, N. Marcantonio, J. F. Anderson, D. Fish, E. Fikrig, and F. S. Kantor. 1998. Prevention of Borrelia burgdorferi transmission in guinea pigs by tick immunity. Am. J. Trop. Med. Hyg. 58: 780-785.

Nilsson, A., and L. Lundqvist. 1978. Host selection and movements of Ixodes ricinus (Acari) larvae on small mammals. Oikos 31: 313-322.

Norte, A. C., A. Alves da Silva, J. Alves, L. P. da Silva, M. S. Núncio, R. Escudero, P. Anda, J. A. Ramos, and I. Lopes de Carvalho. 2015. The importance of lizards and small mammals as reservoirs for Borrelia lusitaniae in Portugal. Environmental Microbiology Reports 7: 188-193.

Novikov, E., E. Kondratyuk, D. Petrovski, A. Krivopalov, and M. Moshkin. 2015. Effects of parasites and antigenic challenge on metabolic rates and thermoregulation in northern red-backed voles (Myodes rutilus). Parasitol Res 114: 4479-4486.

O'Shea, B., E. Rebollar-Tellez, R. D. Ward, J. G. Hamilton, D. el Naiem, and A. Polwart. 2002. Enhanced sandfly attraction to Leishmania-infected hosts. Trans R Soc Trop Med Hyg 96: 117-118.

Osterkamp, J., U. Wahl, G. Schmalfuss, and W. Haas. 1999. Host-odour recognition in two tick species is coded in a blend of vertebrate volatiles. J Comp Physiol 185: 59-67.

Ostfeld, R. S., and F. Keesing. 2000. Pulsed resources and community dynamics of consumers in terrestrial ecosystems. Trends in Ecology and Evolution 15: 232-237.

Ostfeld, R. S., C. G. Jones, and J. O. Wolff. 1996. Of mice and mast. Bioscience 46: 323330.

Ostfeld, R. S., C. D. Canham, K. Oggenfuss, R. J. Winchcombe, and F. Keesing. 2006. Climate, deer, rodents, and acorns as determinants of variation in Lyme disease risk. PLoS Biol. 4: 1058-1068.

Ostfeld, R. S., E. M. Schauber, C. D. Canham, F. Keesing, C. G. Jones, and J. O. Wolff. 2001. Effects of acorn production and mouse abundance on abundance and Borrelia burgdorferi infection prevalence of nymphal Ixodes scapularis ticks. Vector Borne Zoonotic Dis. 1: 55-63.

Pacilly, F. C., M. E. Benning, F. Jacobs, J. Leidekker, H. Sprong, S. E. Van Wieren, and W. Takken. 2014. Blood feeding on large grazers affects the transmission of Borrelia burgdorferi sensu lato by Ixodes ricinus. Ticks Tick Borne Dis 5: 810-817. 
Panzacchi, M., J. D. C. Linnell, C. Melis, M. Odden, J. Odden, L. Gorini, and R. Andersen. 2010. Effect of land-use on small mammal abundance and diversity in a forest-farmland mosaic landscape in south-eastern Norway. For. Ecol. Manage. 259: 1536-1545.

Parola, P., and D. Raoult. 2001. Ticks and tickborne bacterial diseases in humans: An emerging infectious threat. Clin. Infect. Dis. 32: 897-928.

Paul, R. E. L., M. Cote, E. Le Naour, and S. I. Bonnet. 2016. Environmental factors influencing tick densities over seven years in a French suburban forest. Parasit Vectors 9.

Penn, D., G. Schneider, K. White, P. Slev, and W. Potts. 1998. Influenza infection neutralizes the attractiveness of male odour to female mice (Mus musculus). Ethology 104: 685-694.

Perea, R., A. S. Miguel, and L. Gil. 2011. Acorn dispersal by rodents: The importance of redispersal and distance to shelter. Basic Appl. Ecol. 12: 432-439.

Perez, D., Y. Kneubuhler, O. Rais, and L. Gern. 2012. Seasonality of Ixodes ricinus ticks on vegetation and on rodents and Borrelia burgdorferi sensu lato genospecies diversity in two Lyme borreliosis-endemic areas in Switzerland. Vector Borne Zoonotic Dis. 12: 633-644.

Perez, G., S. Bastian, A. Agoulon, A. Bouju, A. Durand, F. Faille, I. Lebert, Y. Rantier, O. Plantard, and A. Butet. 2016. Effect of landscape features on the relationship between Ixodes ricinus ticks and their small mammal hosts. Parasit Vectors 9.

Perkins, S. E., I. M. Cattadori, V. Tagliapietra, A. P. Rizzoli, and P. J. Hudson. 2003. Empirical evidence for key hosts in persistence of a tick-borne disease. Int. J. Parasitol. 33: 909-917.

Perret, J. L., E. Guigoz, O. Rais, and L. Gern. 2000. Influence of saturation deficit and temperature on Ixodes ricinus tick questing activity in a Lyme borreliosis-endemic area (Switzerland). Parasitol Res 86: 554-557.

Perret, J. L., P. M. Guerin, P. A. Diehl, M. Vlimant, and L. Gern. 2003. Darkness induces mobility, and saturation deficit limits questing duration, in the tick Ixodes ricinus. J. Exp. Biol. 206: 1809-1815.

Pichon, B., D. Egan, M. Rogers, and J. Gray. 2003. Detection and Identification of Pathogens and Host DNA in Unfed Host-Seeking Ixodes ricinus L. (Acari: Ixodidae). J. Med. Entomol. 40: 723-731.

Pichon, B., M. Rogers, D. Egan, and J. Gray. 2005. Blood-meal analysis for the identification of reservoir hosts of tick-borne pathogns in Ireland. Vector Borne Zoonotic Dis. 5: 172180.

Pichon, B., E. Godfroid, B. Hoyois, A. Bollen, F. Rodhain, and C. Perez-Eid. 1995. Simultaneous infection of Ixodes ricinus nymphs by two Borrelia burgdorferi sensu lato 
species: possible implications for clinical manifestations. Emerging Infect. Dis. 1: 8990.

Piesman, J. 1991. Experimental acquisition of the Lyme disease spirochete, Borrelia burgdorferi, by larval Ixodes dammini (Acari: Ixodidae) during partial blood meals. J Med Entomol 28: 259-262.

Piesman, J., and R. J. Sinsky. 1988. Ability to Ixodes scapularis, Dermacentor variabilis, and Amblyomma americanum (Acari: Ixodidae) to acquire, maintain, and transmit Lyme disease spirochetes (Borrelia burgdorferi). J. Med. Entomol. 25: 336-339.

Piesman, J., J. R. Oliver, and R. J. Sinsky. 1990. Growth kinetics of the Lyme disease spirochete (Borrelia burgdorferi) in vector ticks (Ixodes dammini). Am. J. Trop. Med. Hyg. 42: 352-357.

Pisanu, B., M. Marsot, J. Marmet, J. L. Chapuis, D. Reale, and G. Vourc'h. 2010. Introduced Siberian chipmunks are more heavily infested by ixodid ticks than are native bank voles in a suburban forest in France. Int. J. Parasitol. 40: 1277-1283.

Platonov, A. E., L. S. Karan, N. M. Kolyasnikova, N. A. Makhneva, M. G. Toporkova, V. V. Maleev, D. Fish, and P. J. Krause. 2011. Humans Infected with Relapsing Fever Spirochete Borrelia miyamotoi, Russia. Emerging Infectious Disease journal 17: 18161823.

Pollock, N. B., L. K. Vredevoe, and E. N. Taylor. 2012. How do host sex and reproductive state affect host preference and feeding duration of ticks? Parasitol Res 111: 897-907.

Poulin, R., and F. Maure. 2015. Host Manipulation by Parasites: A Look Back Before Moving Forward. Trends Parasitol 31: 563-570.

Pucek, Z., W. Jedrzejewski, B. Jedrzejewska, and M. Pucek. 1993. Rodent population dynamics in a primeval deciduous forest (Bialowieza National Park) in relation to weather, seed crop, and predation. Acta Theriol 38: 199-232.

Raberg, L. 2012. Infection intensity and infectivity of the tick-borne pathogen Borrelia afzelii. J. Evol. Biol. 25: 1448-1453.

Radolf, J. D., M. J. Caimano, B. Stevenson, and L. T. Hu. 2012. Of ticks, mice and men: understanding the dual-host lifestyle of Lyme disease spirochaetes. Nat. Rev. Microbiol. 10: 87-99.

Radzijevskaja, J., A. Paulauskas, O. Rosef, S. Petkevicius, V. Mazeika, and T. Rekasius. 2013. The propensity of voles and mice to transmit Borrelia burgdorferi sensu lato infection to feeding ticks. Vet Parasitol 197: 318-325.

Randolph, S. E. 1979. Population regulation in ticks: The role of acquired resistance in natural and unnatural hosts. Parasitology 79: 141-156.

Randolph, S. E. 1991. The effect of Babesia microti on feeding and survival in its tick vector, Ixodes trianguliceps. Parasitology 102: 9-16. 
Randolph, S. E. 1994. Density-dependent acquired resistance to ticks in natural hosts, independent of concurrent infection with Babesia microti. Parasitology 108: 413-419.

Randolph, S. E. 2011. Transmission of tick-borne pathogens between co-feeding ticks: Milan Labuda's enduring paradigm. Ticks Tick Borne Dis 2: 179-182.

Randolph, S. E., and P. A. Nuttall. 1994. Nearly right or precisely wrong - Natural versus laboratory studies of vector-borne diseases. Parasitol Today 10: 458-462.

Randolph, S. E., and N. G. Craine. 1995. General framework for comparative quantitative studies on transmission of tick-borne diseases using lyme borreliosis in Europe as an example. J. Med. Entomol. 32: 765-777.

Randolph, S. E., and K. Storey. 1999. Impact of microclimate on immature tick-rodent host interactions (Acari: Ixodidae): implications for parasite transmission. J Med Entomol 36: 741-748.

Randolph, S. E., L. Gern, and P. A. Nuttall. 1996. Co-feeding ticks: Epidemiological significance for tick-borne pathogen transmission. Parasitol Today 12: 472-479.

Randolph, S. E., R. M. Green, A. N. Hoodless, and M. F. Peacey. 2002. An empirical quantitative framework for the seasonal population dynamics of the tick Ixodes ricinus. Int. J. Parasitol. 32: 979-989.

Randolph, S. E., D. Miklisová, J. Lysy, D. J. Rogers, and M. Labuda. 1999. Incidence from coincidence: Patterns of tick infestations on rodents facilitate transmission of tick-borne encephalitis virus. Parasitology 118: 177-186.

Rauter, C., and T. Hartung. 2005. Prevalence of Borrelia burgdorferi sensu lato genospecies in Ixodes ricinus ticks in Europe: a metaanalysis. Appl. Environ. Microbiol. 71: 72037216.

Rego, R. O., A. Bestor, J. Stefka, and P. A. Rosa. 2014. Population bottlenecks during the infectious cycle of the Lyme disease spirochete Borrelia burgdorferi. PLoS ONE 9: e101009.

Ribeiro, J. M. 1989. Role of saliva in tick/host interactions. Exp Appl Acarol 7: 15-20.

Richter, D., and F. R. Matuschka. 2006. Perpetuation of the Lyme disease spirochete Borrelia Iusitaniae by lizards. Appl. Environ. Microbiol. 72: 4627-4632.

Richter, D., D. B. Schlee, and F. R. Matuschka. 2003. Relapsing fever-like spirochetes infecting European vector tick of Lyme disease agent. Emerging Infect. Dis. 9: 697701.

Richter, D., D. B. Schlee, and F. R. Matuschka. 2011. Reservoir competence of various rodents for the Lyme disease spirochete Borrelia spielmanii. Appl. Environ. Microbiol. 77: 3565-3570.

Richter, D., B. Klug, A. Spielman, and F. R. Matuschka. 2004a. Adaptation of diverse Lyme disease spirochetes in a natural rodent reservoir host. Infect. Immun. 72: 2442-2444. 
Richter, D., D. B. Schlee, R. Allgower, and F. R. Matuschka. 2004b. Relationships of a novel Lyme disease spirochete, Borrelia spielmanii sp nov., with its hosts in central Europe. Appl. Environ. Microbiol. 70: 6414-6419.

Richter, D., A. Debski, Z. Hubalek, and F. R. Matuschka. 2012. Absence of Lyme disease spirochetes in larval Ixodes ricinus ticks. Vector Borne Zoonotic Dis. 12: 21-27.

Rollend, L., D. Fish, and J. E. Childs. 2013. Transovarial transmission of Borrelia spirochetes by Ixodes scapularis: a summary of the literature and recent observations. Ticks Tick Borne Dis 4: 46-51.

Rosa, R., A. Pugliese, M. Ghosh, S. E. Perkins, and A. Rizzoli. 2007. Temporal variation of Ixodes ricinus intensity on the rodent host Apodemus flavicollis in relation to local climate and host dynamics. Vector Borne Zoonotic Dis. 7: 285-295.

Rupprecht, T. A., U. Koedel, V. Fingerle, and H. W. Pfister. 2008. The pathogenesis of lyme neuroborreliosis: From infection to inflammation. Molecular Medicine 14: 205-212.

SAS. 2004. SAS/STAT 9.1 User's Guide, SAS Institute Inc., Cary, NC.

Scantlebury, M., J. M. Waterman, M. Hillegass, J. R. Speakman, and N. C. Bennett. 2007. Energetic costs of parasitism in the Cape ground squirrel Xerus inauris. Proc R Soc Biol Sci Ser B 274: 2169-2177.

Schmidt, K. A., R. S. Ostfeld, and E. M. Schauber. 1999. Infestation of Peromyscus leucopus and Tamias striatus by Ixodes scapularis (Acari: Ixodidae) in relation to the abundance of hosts and parasites. J. Med. Entomol. 36: 749-757.

Schmidt, S., S. S. Essbauer, A. Mayer-Scholl, S. Poppert, J. Schmidt-Chanasit, B. Klempa, K. Henning, G. Schares, M. H. Groschup, F. Spitzenberger, D. Richter, G. Heckel, and R. G. Ulrich. 2014a. Multiple infections of rodents with zoonotic pathogens in Austria. Vector Borne Zoonotic Dis. 14: 467-475.

Schmidt, S., S. S. Essbauer, A. Mayer-Scholl, S. Poppert, J. Schmidt-Chanasit, B. Klempa, K. Henning, G. Schares, M. H. Groschup, F. Spitzenberger, D. Richter, G. Heckel, and R. G. Ulrich. 2014b. Multiple infections of rodents with zoonotic pathogens in Austria. Vector Borne Zoonotic Dis. 14: 467-475.

Schnabel, Z. E. 1983. The estimation of the total fish population of a lake. American Mathematical Monographs 45: 348-352.

Schouls, L. M., I. Van De Pol, S. G. T. Rijpkema, and C. S. Schot. 1999. Detection and identification of Ehrlichia, Borrelia burgdorferi sensu lato, and Bartonella species in Dutch Ixodes ricinus ticks. J. Clin. Microbiol. 37: 2215-2222.

Schuijt, T. J., J. W. Hovius, T. van der Poll, A. P. van Dam, and E. Fikrig. 2011. Lyme borreliosis vaccination: The facts, the challenge, the future. Trends Parasitol 27: 40-47. 
Schwanz, L. E., M. J. Voordouw, D. Brisson, and R. S. Ostfeld. 2011a. Borrelia burgdorferi has minimal impact on the lyme disease reservoir host Peromyscus leucopus. Vector Borne Zoonotic Dis. 11: 117-124.

Schwanz, L. E., D. Brisson, M. Gomes-Solecki, and R. S. Ostfeld. 2011b. Linking disease and community ecology through behavioural indicators: immunochallenge of whitefooted mice and its ecological impacts. J. Anim. Ecol. 80: 204-214.

Schwanz, L. E., M. A. Previtali, M. Gomes-Solecki, D. Brisson, and R. S. Ostfeld. 2012. Immunochallenge reduces risk sensitivity during foraging in white-footed mice. Anim. Behav. 83: 155-161.

Scoles, G. A., M. Papero, L. Beati, and D. Fish. 2001. A relapsing fever group spirochete transmitted by Ixodes scapularis ticks. Vector Borne Zoonotic Dis. 1: 21-34.

Scott, M. C., J. R. Harmon, J. I. Tsao, C. J. Jones, and G. J. Hickling. 2012. Reverse line blot probe design and polymerase chain reaction optimization for bloodmeal analysis of ticks from the eastern United States. J. Med. Entomol. 49: 697-709.

Selås, V., E. Framstad, and T. K. Spidsø. 2002. Effects of seed masting of bilberry, oak and spruce on sympatric populations of bank vole (Clethrionomys glareolus) and wood mouse (Apodemus sylvaticus) in southern Norway. J. Zool. 258: 459-468.

Shaw, M. T., F. Keesing, R. McGrail, and R. S. Ostfeld. 2003. Factors influencing the distribution of larval blacklegged ticks on rodent hosts. Am. J. Trop. Med. Hyg. 68: 447452.

Shih, C. M., and A. Spielman. 1993. Accelerated transmission of Lyme disease spirochetes by partially fed vector ticks. J. Clin. Microbiol. 31: 2878-2881.

Shih, C. M., R. J. Pollack, S. R. Telford, 3rd, and A. Spielman. 1992. Delayed dissemination of Lyme disease spirochetes from the site of deposition in the skin of mice. J. Infect. Dis. 166: 827-831.

Siński, E., R. Welc-Falęciak, and J. Zajkowska. 2016. Borrelia miyamotoi: A human tickborne relapsing fever spirochete in Europe and its potential impact on public health. Advances in Medical Sciences 61: 255-260.

Sinsky, R. J., and J. Piesman. 1989. Ear punch biopsy method for detection and isolation of Borrelia burgdorferi from rodents. J. Clin. Microbiol. 27: 1723-1727.

Slowik, T. J., and R. S. Lane. 2009. Feeding preferences of the immature stages of three western North American Ixodid ticks (Acari) for avian, reptilian, or rodent hosts. 46.

Snydman, D. R., D. P. Schenkein, V. P. Berardi, C. C. Lastavica, and K. M. Pariser. 1986. Borrelia burgdorferi in joint fluid in chronic Lyme arthritis. Ann Intern Med 104: 798-800.

Sonenshine, D. E. 2004. Pheromones and other semiochemicals of ticks and their use in tick control. Parasitology 129: S405-S425. 
Sonenshine, D. E., and J. Stout. 1968. Tick burdens in relation to spacing and range of hosts in Dermacentor variabilis. J. Med. Entomol. 5: 49-52.

Sonenshine, D. E., D. Taylor, and K. A. Carson. 1986. Chemically mediated behavior in Acari: Adapations for finding hosts and mates. J. Chem. Ecol. 12: 1091-1108.

Sormunen, J. J., R. Penttinen, T. Klemola, J. Hänninen, I. Vuorinen, M. Laaksonen, I. E. Sääksjärvi, K. Ruohomäki, and E. J. Vesterinen. 2016. Tick-borne bacterial pathogens in southwestern Finland. Parasit Vectors 9.

Sprong, H., A. Hofhuis, F. Gassner, W. Takken, F. Jacobs, A. J. van Vliet, M. van Ballegooijen, J. van der Giessen, and K. Takumi. 2012. Circumstantial evidence for an increase in the total number and activity of Borrelia-infected Ixodes ricinus in the Netherlands. Parasit Vectors: 294.

Stafford III, K. C., M. L. Cartter, L. A. Magnarelli, S. H. Ertel, and P. A. Mshar. 1998. Temporal correlations between tick abundance and prevalence of ticks infected with Borrelia burgdorferi and increasing incidence of Lyme disease. J. Clin. Microbiol. 36: 1240-1244.

Stanek, G., and F. Strle. 2003. Lyme borreliosis. Lancet 362: 1639-1647.

Stanek, G., G. P. Wormser, J. Gray, and F. Strle. 2012. Lyme borreliosis. Lancet 379: 461473.

Stein, R. A. 2011. Super-spreaders in infectious diseases. International Journal of Infectious Diseases 15: e510-e513.

Stradiotto, A., F. Cagnacci, R. Delahay, S. Tioli, L. Nieder, and A. Rizzoli. 2009. Spatial organization of the yellow-necked mouse: Effects of density and resource availability. J. Mammal. 90: 704-714.

Strle, F., and G. Stanek. 2009. Clinical manifestations and diagnosis of lyme borreliosis. Curr Probl Dermatol 37: 51-110.

Strube, C., V. M. Montenegro, C. Epe, E. Eckelt, and T. Schnieder. 2010. Establishment of a minor groove binder-probe based quantitative real time PCR to detect Borrelia burgdorferi sensu lato and differentiation of Borrelia spielmanii by ospA-specific conventional PCR. Parasit Vectors 3: 69.

Sunyer, P., A. Muñoz, M. J. Mazerolle, R. Bonal, and J. M. Espelta. 2016. Wood mouse population dynamics: Interplay among seed abundance seasonality, shrub cover and wild boar interference. Mamm Biol 81: 372-379.

Szekeres, S., E. C. Coipan, K. Rigó, G. Majoros, S. Jahfari, H. Sprong, and G. Földvári. 2015. Eco-epidemiology of Borrelia miyamotoi and Lyme borreliosis spirochetes in a popular hunting and recreational forest area in Hungary. Parasit Vectors.

Takabayashi, J., and M. Dicke. 1992. Response of predatory mites with different rearing histories to volatiles of uninfested plants. Entomol. Exp. Appl. 64: 187-193. 
Takken, W., and D. L. Kline. 1989. Carbon dioxide and 1-octen-3-ol as mosquito attractants. J. Am. Mosq. Control Assoc. 5: 311-316.

Takken, W., and N. O. Verhulst. 2013. Host preferences of blood-feeding mosquitoes. Annu. Rev. Entomol. 58: 433-453.

Tälleklint, L., and T. G. T. Jaenson. 1994. Transmission of Borrelia burgdorferi s.l. from mammal reservoirs to the primary vector of lyme borreliosis, Ixodes ricinus (Acari: Ixodidae), in Sweden. J. Med. Entomol. 31: 880-886.

Tälleklint, L., and T. G. Jaenson. 1995. Is the small mammal (Clethrionomys glareolus) or the tick vector (Ixodes ricinus) the primary overwintering reservoir for the Lyme borreliosis spirochete in Sweden? J. Wildl. Dis. 31: 537-540.

Tälleklint, L., and T. G. Jaenson. 1997. Infestation of mammals by Ixodes ricinus ticks (Acari: Ixodidae) in south-central Sweden. Exp Appl Acarol 21: 755-771.

Tappe, J., D. Jordan, E. Janecek, V. Fingerle, and C. Strube. 2014. Revisited: Borrelia burgdorferi sensu lato infections in hard ticks (Ixodes ricinus) in the city of Hanover (Germany). Parasit Vectors 7: 441.

Taylor, K. R., A. Takano, S. Konnai, M. Shimozuru, H. Kawabata, and T. Tsubota. 2013a. Borrelia miyamotoi infections among wild rodents show age and month independence and correlation with Ixodes persulcatus larval attachment in Hokkaido, Japan. Vector Borne Zoonotic Dis. 13: 92-97.

Taylor, K. R., A. Takano, S. Konnai, M. Shimozuru, H. Kawabata, and T. Tsubota. 2013b. Differential tick burdens may explain differential Borrelia afzelii and Borrelia garinii infection rates among four, wild, rodent species in Hokkaido, Japan. J. Vet. Med. Sci. 75: 785-790.

Thomas, F., S. Adamo, and J. Moore. 2005. Parasitic manipulation: where are we and where should we go? Behav. Processes 68: 185-199.

Tonetti, N., M. J. Voordouw, J. Durand, S. Monnier, and L. Gern. 2015. Genetic variation in transmission success of the Lyme borreliosis pathogen Borrelia afzelii. Ticks Tick Borne Dis 6: 334-343.

Trager, W. 1939. Acquired immunity to ticks. The Journal of Parasitology 25: 57-81.

Tschirren, B., M. Andersson, K. Scherman, H. Westerdahl, P. R. Mittl, and L. Raberg. 2013. Polymorphisms at the innate immune receptor TLR2 are associated with Borrelia infection in a wild rodent population. Proc Biol Sci 280: 20130364.

Van Duijvendijk, G., H. Sprong, and W. Takken. 2015. Multi-trophic interactions driving the transmission cycle of Borrelia afzelii between Ixodes ricinus and rodents: a review. Parasit Vectors 8: 643. 
Van Duijvendijk, G., C. Coipan, A. Wagemakers, M. Fonville, J. Ersoz, A. Oei, G. Foldvari, J. Hovius, W. Takken, and H. Sprong. 2016. Larvae of Ixodes ricinus transmit Borrelia afzelii and B. miyamotoi to vertebrate hosts. Parasit Vectors 9: 97.

Van Houte, S., V. I. D. Ros, and M. M. Van Oers. 2013. Walking with insects: Molecular mechanisms behind parasitic manipulation of host behaviour. Mol Ecol 22: 3458-3475.

Voordouw, M. J. 2015. Co-feeding transmission in Lyme disease pathogens. Parasitology 142: 290-302.

Voordouw, M. J., S. Lachish, and M. C. Dolan. 2015. The lyme disease pathogen has no effect on the survival of its rodent reservoir host. PLoS ONE 10: e0118265.

Wagemakers, A., P. J. Staarink, H. Sprong, and J. W. Hovius. 2015. Borrelia miyamotoi: a widespread tick-borne relapsing fever spirochete. Trends Parasitol 31: 260-269.

Wagemakers, A., A. Oei, M. M. Fikrig, W. R. Miellet, and J. W. Hovius. 2014. The relapsing fever spirochete Borrelia miyamotoi is cultivable in a modified Kelly-Pettenkofer medium, and is resistant to human complement. Parasit Vectors 7: 418.

Webster, B., E. S. Lacey, and R. T. Carde. 2015. Waiting with bated breath: Opportunistic orientation to human odor in the malaria mosquito, Anopheles gambiae, is Modulated by Minute Changes in Carbon Dioxide Concentration. J. Chem. Ecol. 41: 59-66.

Wikel, S. K. 1996. Host immunity to ticks. Annu. Rev. Entomol. 41: 1-22.

Wikel, S. K. 1999. Tick modulation of host immunity: an important factor in pathogen transmission. Int. J. Parasitol. 29: 851-859.

Wikel, S. K., R. N. Ramachandra, D. K. Bergman, T. R. Burkot, and J. Piesman. 1997. Infestation with pathogen-free nymphs of the tick Ixodes scapularis induces host resistance to transmission of Borrelia burgdorferi by ticks. Infect. Immun. 65: 335-338.

Wilhelmsson, P., P. Lindblom, L. Fryland, J. Ernerudh, P. Forsberg, and P. E. Lindgren. 2013. Prevalence, diversity, and load of Borrelia species in ticks that have fed on humans in regions of Sweden and Aland Islands, Finland with different Lyme borreliosis incidences. PLoS One 8: e81433.

Willis, C., and R. Poulin. 2000. Preference of female rats for the odours of non-parasitised males: The smell of good genes? Folia Parasitol. 47: 6-10.

Woolhouse, M. E. J., C. Dye, J. F. Etard, T. Smith, J. D. Charlwood, G. P. Garnett, P. Hagan, J. L. K. Hii, P. D. Ndhlovu, R. J. Quinnell, C. H. Watts, S. K. Chandiwana, and R. M. Anderson. 1997. Heterogeneities in the transmission of infectious agents: Implications for the design of control programs. Proc Natl Acad Sci U S A 94: 338-342.

Wright, S. D., and S. W. Nielsen. 1990. Experimental infection of the white-footed mouse with Borrelia burgdorferi. Am. J. Vet. Res. 51: 1980-1987.

Zhong, J., A. Jasinskas, and A. G. Barbour. 2007. Antibiotic Treatment of the Tick Vector Amblyomma americanum Reduced Reproductive Fitness. PLoS ONE 2: e405. 
Zielinski, W. J., and J. G. Vandenbergh. 1993. Testosterone and competitive ability in male house mice, Mus musculus - Laboratory and field studies. Anim. Behav. 45: 873-891.

Zore, A., M. Petrovec, K. Prosenc, T. Trilar, E. Ruzic-Sabljic, and T. Avsic-Zupanc. 1999. Infection of small mammals with Borrelia burgdorferi sensu lato in Slovenia as determined by polymerase chain reaction (PCR). Wien Klin Wochenschr 111: 997-999.

Zug, R., and P. Hammerstein. 2015. Bad guys turned nice? A critical assessment of Wolbachia mutualisms in arthropod hosts. Biol Rev 90: 89-111.

Zwolak, R., M. Bogdziewicz, and L. Rychlik. 2016. Beech masting modifies the response of rodents to forest management. For. Ecol. Manage. 359: 268-276. 



\section{Summary}


The sheep tick (Ixodes ricinus) is widespread throughout Europe and it can transmit Borrelia burgdorferi sensu lato (s.I.), which can cause Lyme borreliosis in humans and B. miyamotoi, which can cause Borrelia miyamotoi disease in humans, as well as several other pathogenic agents. In this thesis, B. burgdorferi s.I. and B. miyamotoi have been studied. Lyme borreliosis is the most common vector-borne disease in the northern hemisphere, whereas Borrelia miyamotoi disease was discovered only recently. Borrelia burgdorferi s.l. consists of several genospecies, which cause varying clinical manifestations in humans and circulate in different enzootic cycles. Borrelia afzelii is the most common genospecies in humans and ticks and it circulates between $I$. ricinus and rodents. It is generally assumed that $I$. ricinus larvae are free of $B$. afzelii (but see Chapter 8), and that they become infected during a blood meal from an infected rodent. The infected larvae moult into infected nymphs, which can transmit the spirochaetes to rodents, completing the enzootic lifecycle of $B$. afzelii. Borrelia miyamotoi, however, can be transmitted transovarially and the importance of rodents for the maintenance of its enzootic cycle is not clear. Wood mice (Apodemus sylvaticus) and bank voles (Myodes glareolus) are the most common rodent species in The Netherlands and are commonly used as hosts by larval $I$. ricinus. The density of $B$. burgdorferi s.I.-infected nymphs (DIN) determines disease risk and affects disease incidence in humans. The DIN is affected by complex interactions between several factors, which are not well understood. The aim of the thesis was, therefore, to gain insight into the multi-trophic interactions between factors that affect the densities of $B$. afzelii-infected and $B$. miyamotoi-infected nymphs. The chapters in this thesis describe several field and laboratory experiments.

In Chapter 2, current knowledge on the multi-trophic interactions that drive the transmission cycle of $B$. afzelii between I. ricinus and rodents is reviewed. Lyme borreliosis risk is determined by the DIN. Borrelia afzelii is the most common genospecies and humans mainly become infected via the bite of infected nymphs. The study focussed, therefore, on the development from questing larva to $B$. afzelii-infected nymph. This development was divided into several steps: 1) larval host finding, 2) larval blood feeding, 3) spirochaete transmission from rodent to larva, and 4) development from larva to nymph. Nymphal host-finding success, nymphal blood-feeding success, and $B$. afzelii transmission from nymph to rodent are the final steps that complete the enzootic $B$. afzelii lifecycle and were included in the review. The effects of host, tick and $B$. afzelii on these factors are discussed. The review shows the various factors that contribute to the density of $B$. afzelii-infected nymphs, and how these factors interact. We suggest that the $B$. afzelii lifecycle is dependent on the aggregation of ticks among rodents, which is manipulated by the pathogen itself. The review demonstrates that rodents are used as host by $I$. ricinus larvae and serve as reservoir host for $B$. afzelii. Rodent density is, 
therefore, assumed to affect the density of infected nymphs one year later, but experimental evidence for this assumption was lacking.

The aim of Chapter 3 was to determine the effect of rodent density on the DIN. Rodent density was manipulated by either removing rodents from an area or supplementing the rodents' natural food. Control plots received no treatment. Rodent density and rodent infection rate were determined every three months for 28 months. DIN and nymphal infection prevalence were determined monthly for 40 months. Rodent density had a positive effect on the DIN one year later. The effect was caused by an increase in the density of nymphs, but not an increase in nymphal infection prevalence. This chapter showed the importance of rodents as blood hosts for $I$. ricinus larvae and their contribution to the DIN. The specific characteristics of a rodent (species, sex) may, however, affect the suitability of a rodent as host for $I$. ricinus and $B$. afzelii. In addition, the contribution of rodents to the DIN may also vary throughout the year.

The aim of Chapter 4 was to identify the factors that affect the contribution of rodents to DIN. The interactions between rodents, I. ricinus, B. burgdorferi s.I. and B. miyamotoi were studied in a natural ecosystem. Rodents were live-trapped and rodent species, sex, weight, identification number, location, time, infection, infectivity and tick burden were determined. We found higher larval and nymphal tick burdens on wood mice than on bank voles and on males compared to females. Larval tick burden and B. burgdorferi s.I. rodent infection rate peaked in summer. Even though rodent density was one order of magnitude higher in 2014 than in 2013 , the contribution of the 2014 rodent population to the DIN was only slightly higher than that of the 2013 rodent population. This was caused by a lower larval and nymphal tick burden and B. burgdorferi s.l. rodent infection rate in 2014 than in 2013. The contribution of the rodent population to the density of $B$. miyamotoi-infected nymphs did not differ between years. We concluded that the DIN and density of $B$. miyamotoi-infected nymphs are not directly affected by a temporal variation in rodent density. This study also showed that larvae are highly aggregated among rodents. There were many rodents with only a few larvae and few rodents feeding the majority of ticks. There are several examples of microorganisms that can affect their host or vector to enhance their own transmission and there are indications that larval tick burdens are higher on infected rodents than on uninfected rodents, which enhances the transmission cycle. Experimental evidence for this hypothesis is, however, lacking.

The aim of Chapter 5 was to determine whether a $B$. afzelii infection enhances its transmission between rodents and $I$. ricinus. We studied two aspects of the transmission cycle: larval tick burden on rodents (affecting transmission from rodent to larva) and larval blood meal size (affecting nymph survival and, therefore, transmission from nymph to rodent). Bank voles were 
bred in captivity and were inoculated with $B$. afzelii or control medium. Host preference of $I$. ricinus nymphs was determined in a Y-tube olfactometer. Infected and uninfected bank voles were placed pairwise in a semi-field arena containing questing larvae to determine the effect of a $B$. afzelii infection in rodents on the acquisition of larval tick burdens. Larvae that attached to the bank voles in the semi-field arena were allowed to engorge and the engorged larvae were collected and allowed to moult into nymphs. The emerged nymphs were weighed and analysed for infection to determine whether a $B$. afzelii infection had an effect on nymphal body weight. The results showed that a $B$. afzelii infection in rodents increased larval tick burden and that a $B$. afzelii infection in nymphs increased nymphal body weight. These two factors enhance the enzootic transmission cycle of $B$. afzelii, resulting in a $27 \%$ higher nymphal infection prevalence.

The contribution of a rodent to the tick and $B$. afzelii populations not only depends on the number of ticks feeding on it, but also on the physiology of the ticks that feed on this rodent. In Chapter 6, an experiment is described to determine the effect of rodent species on larval blood meal size and interstadial development from larva to nymph. Rodents were trapped in the field and transported to the laboratory and the engorged larvae were collected and analysed. Larval blood meal size and body weight of emerged nymphs were determined. Body weight of engorged larvae and body weight of emerged nymphs were 21 and $36 \%$ higher, respectively, for ticks that fed on wood mice compared to ticks that fed on bank voles.

Ixodes ricinus quests in the vegetation until it encounters a host. During questing, the ticks sense $\mathrm{CO}_{2}$ and host odour to detect their host. The differential effect of $\mathrm{CO}_{2}$ and host odour on the host finding behaviour of $I$. ricinus has, however, never been clarified. The aim of the study described in Chapter 7 was, therefore, to investigate the differential effect of $\mathrm{CO}_{2}$ and host odour on the host-seeking behaviour of I. ricinus. The effect of $\mathrm{CO}_{2}$ and host odour on the activation and attraction of $I$. ricinus nymphs was analysed in a Y-tube olfactometer. Nymphal response and preference were measured. Carbon dioxide activated nymphs in the absence and presence of host odour, but did not attract nymphs. Host odour, however, did not activate nymphs but attracted nymphs in the absence and presence of $\mathrm{CO}_{2}$.

It is known that $B$. miyamotoi can be transmitted transovarially from female ticks to larvae. Borrelia burgdorferi s.I. has rarely been found in questing larvae. The ability of larvae to transmit these spirochaetes has, however, never been investigated. Chapter 8 describes a study that examined whether larvae of $I$. ricinus can transmit $B$. burgdorferi s.l. and $B$. miyamotoi to vertebrate hosts. Questing larvae $(28,000)$ were collected from the vegetation at five different field locations around Wageningen, The Netherlands. These larvae were placed 
in batches on laboratory mice. Mice and nymphs emerged from the engorged larvae were analysed for infections with $B$. burgdorferi s.I. and B. miyamotoi by culture and PCR after the experiment.

We showed that field-collected I. ricinus larvae were naturally infected with B. burgdorferi s.I. and $B$. miyamotoi. These larvae transmitted $B$. afzelii and $B$. miyamotoi to rodents and we calculated that rodents have a chance of about $25-75 \%$ to acquire $B$. afzelii from larvae relative to the chance of acquiring $B$. afzelii from nymphs. Chapter 9 summarizes and discusses the main conclusions of this thesis and ends with suggestions for future research.

It is concluded that:

1) Rodent density affects the DIN one year later when questing larvae are not the limiting factor.

2) The aggregation of $I$. ricinus larvae on only a few members of a rodent population is caused by variations in host characteristics (e.g. species, sex, body weight, infection status)

3) Wood mice have larger tick burdens and larvae take a larger blood meal from wood mice than bank voles.

4) Ixodes ricinus is activated by $\mathrm{CO}_{2}$ and attracted to host odour.

5) Borrelia afzelii increases larval tick burden on bank voles and nymphal body weight of $I$. ricinus.

6) Ixodes ricinus larvae transmit $B$. afzelii and $B$. miyamotoi to rodents. 



\section{Appendices}

\section{Appendix 1}
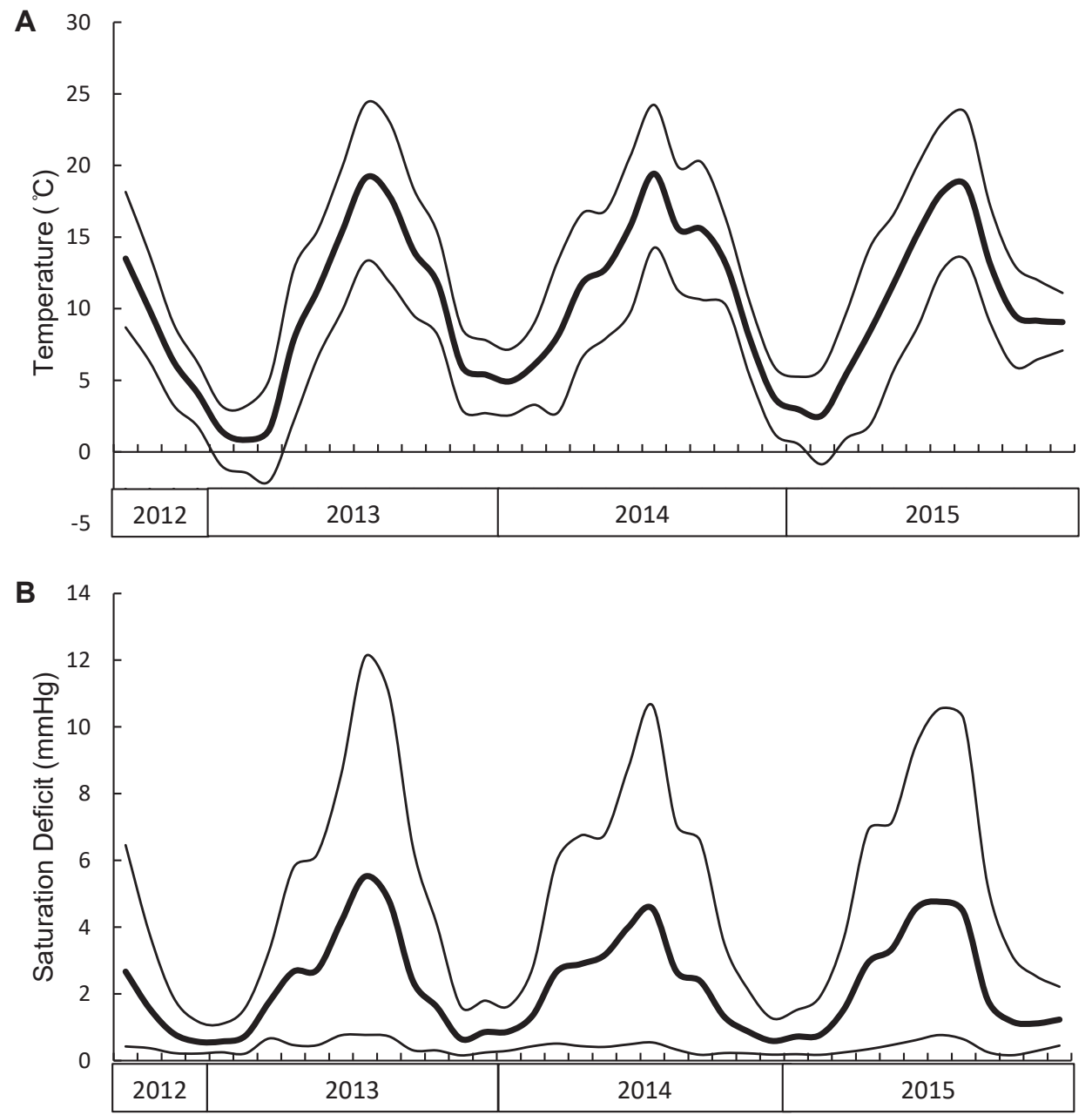

Temperature (A) and saturation deficit $(B)$ throughout the study period. Thick lines represent monthly means. Thin lines represent monthly means of the daily maximum and minimum values. Data based on hourly measurements from weather station 'Deelen', KNMI, The Netherlands. 


\section{Appendix 2}

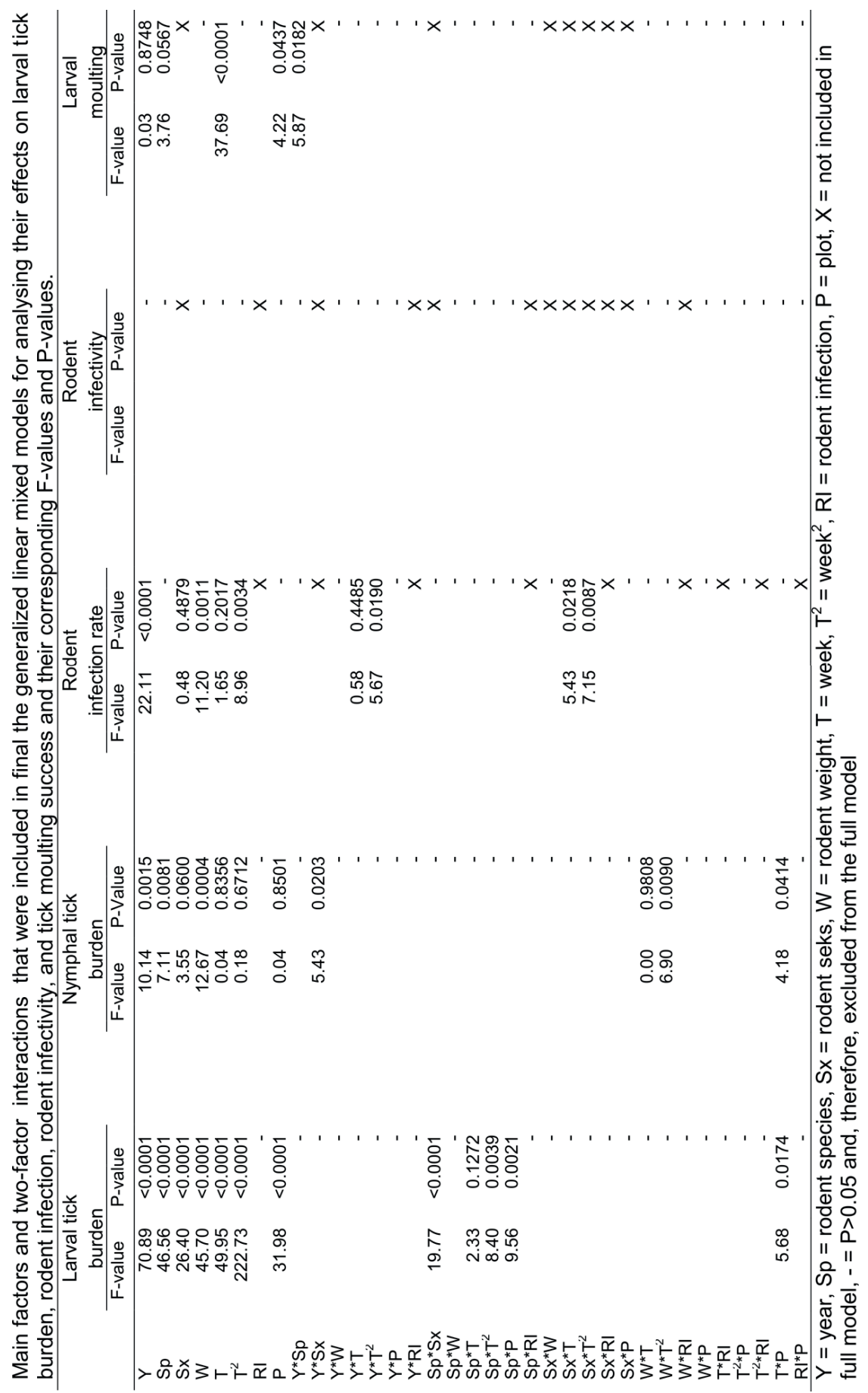




\section{Acknowledgements}

Zo, ik ben klaar met mijn proefschrift. Zonder hulp van anderen was dit proefschrift er niet geweest. Ik wil iedereen die me heeft geholpen daarom graag bedanken.

Ten eerste wil ik graag mijn promotor Willem Takken bedanken voor de prettige samenwerking de afgelopen jaren. Ik waardeer het vertrouwen dat je in me hebt heel erg en ben blij dat je me vaak m'n gang liet gaan. De input die je op mijn onderzoeksplannen en manuscripten had hebben dit proefschrift tot een hoger niveau gebracht.

Daarnaast wil ik ook mijn co-promotor Hein Sprong bedanken voor de fijne samenwerking. Je doelgerichtheid en efficiëntie spreken me erg aan en hebben er voor gezorgd dat de meeste van mijn manuscripten een heldere boodschap hebben. De discussies over mijn experimenten heb ik erg gewaardeerd.

Ik wil ook iedereen van het Shooting the Messenger project bedanken. Claudia en Tim: I enjoyed working in this project and to discuss our experiments during our meetings. Sip, ook jou wil ik graag bedanken voor je nuchtere input tijdens de discussies en Marieta voor je scherpe opmerkingen over de experimenten en resultaten. Manoj en anderen, bedankt voor de vele uren die jullie in het laboratorium hebben gestaan om mijn dozen vol monsters te analyseren. Frans, jou wil ik natuurlijk bedanken voor alle hulp bij het veldwerk en diverse andere werkzaamheden.

Alle studenten die me hebben geholpen met het verzamelen van data ben ik natuurlijk ook heel erg dankbaar. Marloes en Inge bedankt voor de hulp bij de veldexperimenten en het zeulen met kratten met muizenvallen in weer en wind. Wouter bedankt voor de hulp bij de gedragsexperimenten. Nandi, Floor, Anne en Alejandro heel erg bedankt voor de hulp bij de experimenten m.b.t de biologische bestrijding van teken en $B$. afzelii overdracht van muizen naar teken. Helaas ging dat niet altijd even makkelijk en staan deze resultaten niet in m'n proefschrift. De samenwerking en begeleiding van jullie allen vond ik misschien wel het leukste van de afgelopen 4 jaar.

There have been too many colleagues at entomology to thank all by name, but I would like to thank all of you for creating such a nice environment. Marcel bedankt voor de fijne sfeer, Hans, Patrick, Jeroen, Leon en Rieta bedankt voor het verzamelen van alle materialen voor het bouwen van diverse onderzoeksopstellingen en de vele $\mathrm{PhD}$ studenten voor alle gezelligheid. 
Marjolein, en op het eind ook Cindy, bedankt dat ons hoekje het gezelligste plekje van de open werkruimte was. Ik wil ook iedereen van de vectorgroep bedanken. Vooral Willem, Sander, Jetske, Niels, Jeroen, Alex, Leon, David, Tobias, Tim, Claudio en Chantal, bedankt voor de meetings en input op mijn plannen en resultaten. Ook bedankt voor de dagen en nachten dat jullie mee wilden met muizen vangen. Wat was het soms koud... Françoise, bedankt voor de heerlijke chocolademelk tijdens deze koude nachten. I would like to thank Claudio for his help with the biological control experiments. Jeroen en Leon, bedankt dat jullie zonder twijfel mijn paranimfen wilden zijn op deze speciale dag.

Joppe, Alex, Jeroen, Jos, Joris, Jasmin en Anneke van het AMC wil ik bedanken voor hun adviezen, voor de gekweekte $B$. afzelii en tekenlarfjes die ik nodig had voor m'n experimenten en voor de analyse van samples.

Rob en Marijke van de dierexperimentencommissie, Rene, Bert en Wilma van het centrum kleine proefdieren, bedankt voor jullie hulp bij de organisatie van de experimenten en diverse ethische beoordelingen en het geduld bij al mijn last-minute wijzigingen. Andries, bedankt voor de hulp bij de controle van de muizen in de weekenden.

Gerrit, hartelijk dank voor de vele uren die je samen met me in de statistische analyses van de data hebt gestoken.

Han van Natuurmonumenten, Jochem van de gemeente Ede, Bart van Staatsbosbeheer en Robert van het Geldersch Landschap bedankt voor de toestemming om mijn veldexperimenten in de door jullie beheerde gebieden uit te kunnen voeren.

Seta, Jeroen en Tim, wil ik bedanken voor het samen organiseren van het gezellige en succesvolle Tick Tactics symposium.

Alle familie en vrienden, bedankt voor de steun de afgelopen jaren. M'n ouders extra bedankt voor de steun en interesse. Hans en Annemarie bedankt voor het oppassen en de flexibiliteit daarin tijdens mijn experimenten. Robert, jou wil ik bedanken voor de diverse ontspannende lunchwandelingen en positieve discussies over het belang van de wetenschap.

Tot slot wil ik de belangrijkste personen uit mijn leven bedanken: Hannah, je staat altijd voor me klaar en toont altijd veel begrip als ik voor experimenten 's nachts of in het weekend het bos in moet. Ik heb heel veel aan al je hulp gehad en had dit niet zonder jou kunnen doen. Sterker nog, zonder jou was ik er niet eens aan begonnen... Ik ben je ook heel dankbaar dat je tijdens jouw en mijn promotie twee keer zwanger wilde te zijn, terwijl dit regelmatig onmogelijk met het werk te combineren was. Dagmar en Laurens, jullie wil ik bedanken dat ik jullie vader mag zijn.

Bedankt! 


\section{Curriculum vitae}

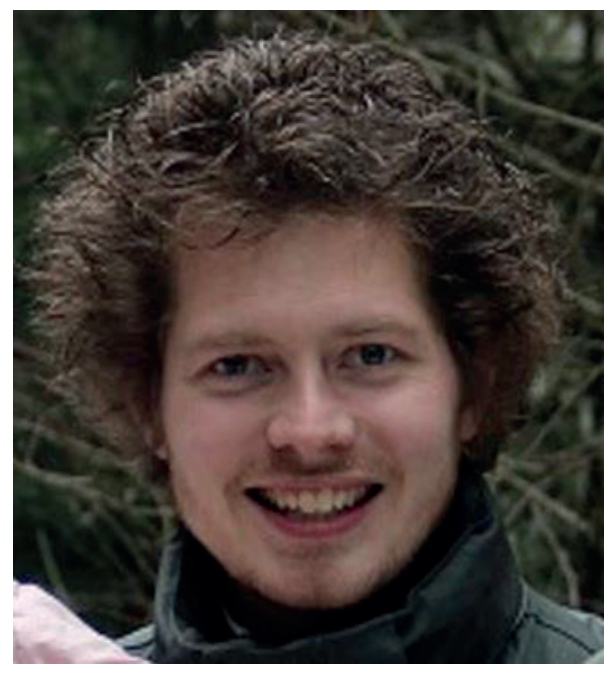

Gilian van Duijvendijk was born in Groningen, The Netherlands, on 18 May 1983. He grew up in Zuidwolde and went to secondary school in Hoogeveen. He acquired his bachelor degree in Animal Management (Specialisation: Wildlife management) at the university of applied sciences (Van Hall Larenstein) in Leeuwarden. $\mathrm{He}$ continued with an MSc in Animal Sciences at Wageningen University. During his MSc he specialised in Animal Health and Behaviour and Entomology. The topic of his first thesis was "Gas exchange during bird egg incubation" and the topic of his second thesis was "Effect of a Lyme borreliosis bacterium infection on tick behaviour and survival". After finishing his MSc, he worked for several months as a research assistant at the Laboratory of Entomology on the ecology and biological control of red poultry mites. He then worked for two years as a junior researcher at the Nature Conservation and Plant Ecology Group at Wageningen University in a project on the effect of infrastructure on the ecology of insects. In 2012 he started his PhD at the Laboratory of Entomology on the interactions between ticks, rodents and Lyme borreliosis bacteria. During his experience as research assistant, junior researcher and PhD candidate, he was very enthusiastic about the supervision of BSc and MSc students. As of September 2016, Gilian is employed as a teacher and advisor in entomology at the university of applied sciences (HAS) in Den Bosch. 


\section{Training and Education Statement PE\&RC}

With the training and education activities listed below the $\mathrm{PhD}$ candidate has complied with the requirements set by the C.T. de Wit Graduate School for Production Ecology and Resource Conservation (PE\&RC), which comprises of a minimum total of 32 ECTS (= 22 weeks of activities)

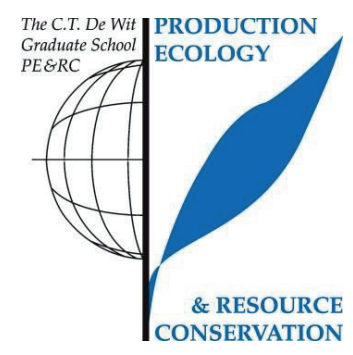

\section{Review of literature (6 ECTS)}

- Multi-trophic interactions driving the transmission cycle of Borrelia afzelii between Ixodes ricinus and rodents

\section{Writing of project proposal (4.5 ECTS)}

- Control of tick-borne diseases: Shooting the messenger

\section{Post-graduate courses (7.6 ECTS)}

- $\quad$ Spatial ecology; PE\&RC (2011)

- Linear models; PE\&RC (2012)

- Mixed linear models; PE\&RC (2012)

- Generalized linear models; PE\&RC (2015)

- The science of conservation, managing biodiversity in a changing world; PE\&RC, University of Venda, South Africa (2016)

\section{Laboratory training and working visits (0.1 ECTS)}

- Borrelia breeding; AMC, Amsterdam (2012) 


\section{Competence strengthening / skills courses (5.3 ECTS)}

- Competence assessment; Maas Organisation \& Career Development, Amersfoort (2011)

- Scientific publishing; WGS (2011)

- Project and time management; Valley Consult (2011)

- Stress identification and management; WGS (2012)

- Techniques for writing and presenting a scientific paper; WGS (2015)

- Writing grand proposals; WGS (2015)

- Brain training; De Hoofdzaken (2015)

\section{PE\&RC Annual meetings, seminars and the PE\&RC weekend (1.5 ECTS)}

- PE\&RC Day (2010-2012, 2014, 2015)

\section{Discussion groups / local seminars / other scientific meetings (10.2 ECTS)}

- Ecological theory and application (2010-2015)

- Entomologendag (2012-2015)

- PhD Literature discussion group (2012-2016)

- Vector discussion group (2012-2016)

- Vectory (2014)

- Wageningen evolution \& ecology seminar (2014-2016)

- PhD Symposium, connecting ideas, combining forces (2015)

- Discussion Laboratory for Zoonosis and Environmental Microbiology, National Institute for Public Health and environment (2015-2016)

- Global one health symposium (2016)

\section{International symposia, workshops and conferences (9.6 ECTS)}

- $\quad \mathrm{XII}^{\text {th }}$ International Jena symposium on tick-borne diseases (2013)

- Netherlands annual ecology meeting (2013-2015)

- Tick tactics symposium (2014)

- Zoology congress (2015)

\section{Lecturing / supervision of practicals / tutorials (9.9 ECTS)}

- Behavioural ecology (2012) 
- Basics of infectious diseases (2012-2015)

- Ecology (2013-2016)

- Molecular and evolutionary ecology (2015)

\section{Supervision of 6 MSc students (18 ECTS)}

- Effect of rodent odour on Ixodes ricinus behaviour

- Rodents as host for Ixodes ricinus and Borrelia afzelii

- Control of Ixodes ricinus with entomopathogenic fungi

- The contribution of rodents to Lyme borreliosis risk

- Does Borrelia afzelii affect its own transmission?

- Control of Ixodes ricinus with entomopathogenic fungi 

Thesis layout:

Cover design:

Cover picture:
Gilian van Duijvendijk

Gilian van Duijvendijk

Hans Smid / Leon Westerd / Gilian van Duijvendijk 